\title{
Charakterisierung Und Optimierung VON DIFFUSIONSBARRIEREN \\ AUF DER BASIS METALLISCHER OXIDSCHICHTEN
}

\author{
Dissertation zur Erlangung des Doktorgrades \\ der Mathematisch-Naturwissenschaftlichen Fakultäten \\ der Georg-August-Universität zu Göttingen
}

\section{VORGELEGT VON}

Cay-Uwe Pinnow

aus Kiel

Göttingen 2001 
D7

Referent: Prof. Dr. K. Samwer

Korreferent: Priv. Doz. Dr. M. Seibt

Tag der mündlichen Prüfung: 11.12.2001 


\section{Inhaltsverzeichnis}

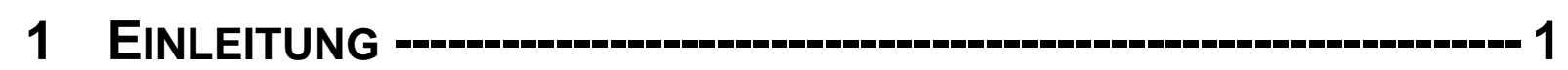

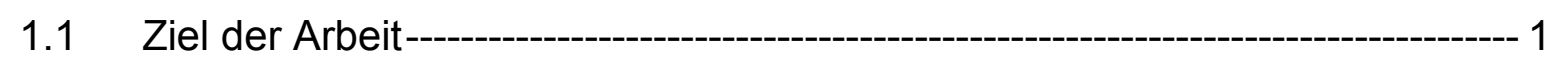

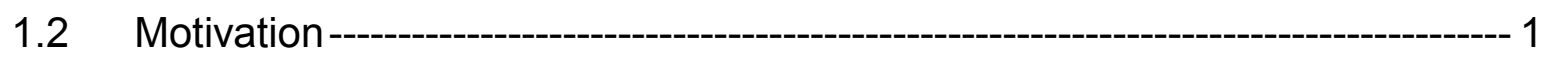

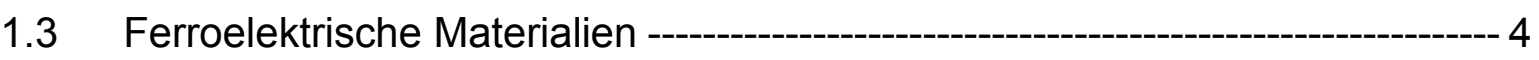

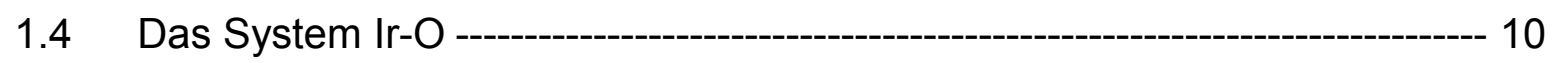

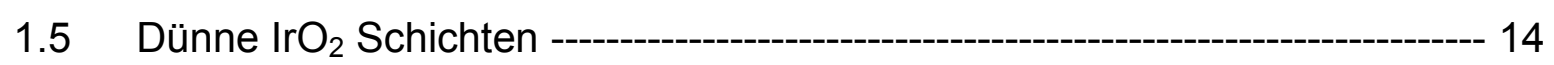

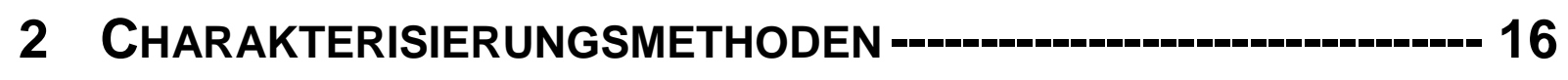

2.1 Prozess-Überwachung -------------------------------------------------------------------- 16

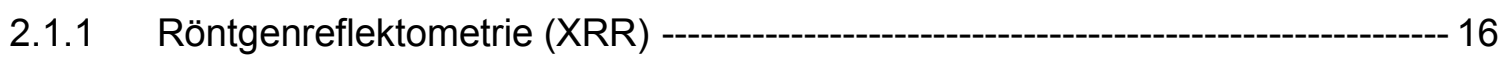

$2.1 .2 \quad$ Profilometrie $-\cdot-\cdot--\cdot-\cdot-\cdot-\cdot-17$

$2.1 .3 \quad$ Vierpunkts-Schichtwiderstandsmessungen-------------------------------------- 17

2.1.4 Messungen der mechanischen Schichtspannungen-------------------------------- 18

2.2 Stöchiometrie, Chemische Analyse, Tiefenprofilanalysemethoden ---------- 19

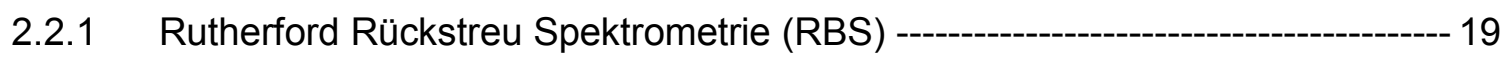

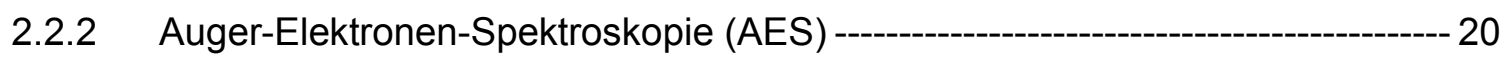

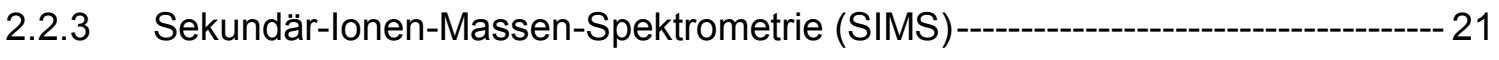

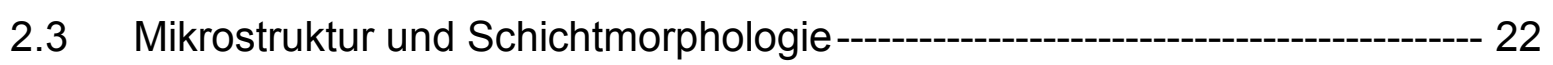

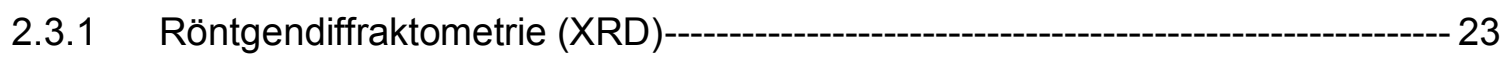

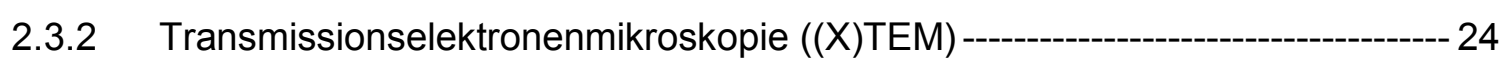

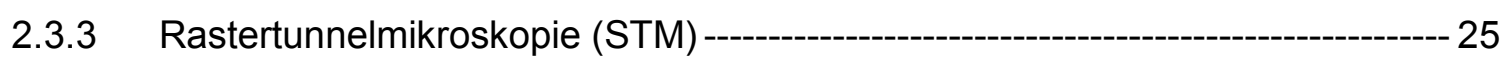

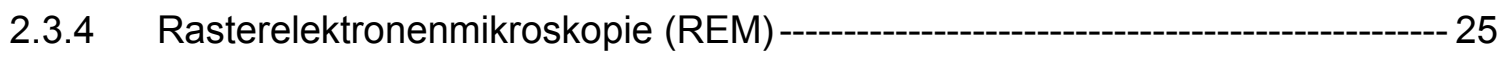

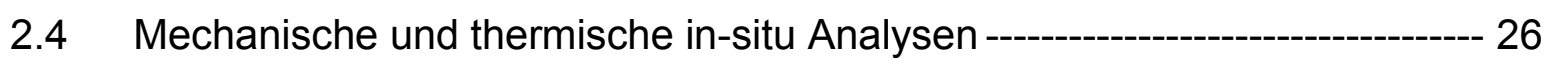


$2.4 .1 \quad$ In-situ-Messungen der mechanischen Schichtspannungen ---------------------- 26

2.4.2 Differentielle Thermische Analyse (DTA) mit simultaner Restgasanalyse (RGA) und thermogravimetrischer Analyse (TGA) ----------------------------------------26

\section{SCHICHTDEPOSITION}

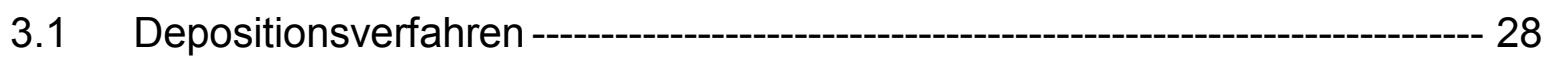

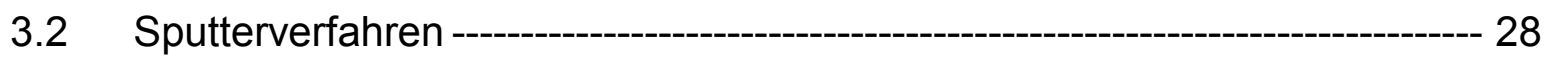

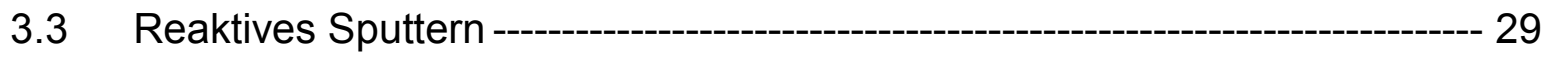

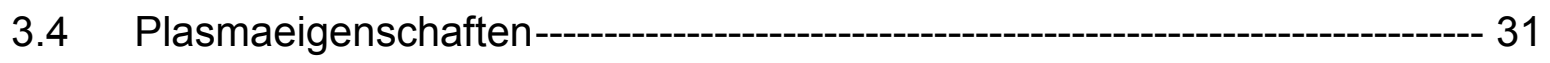

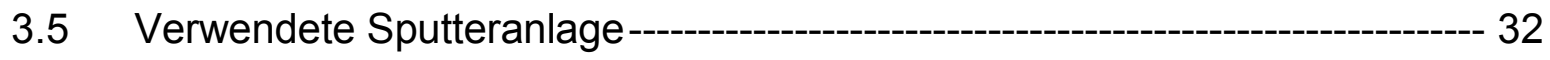

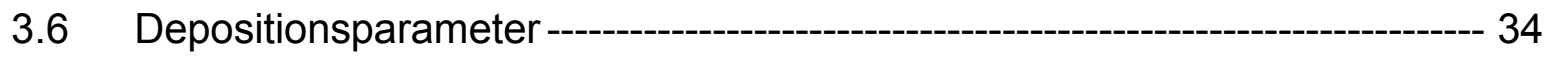

3.7 Optimierung des Depositionsprozesses mittels generischer Kurven -------- 36

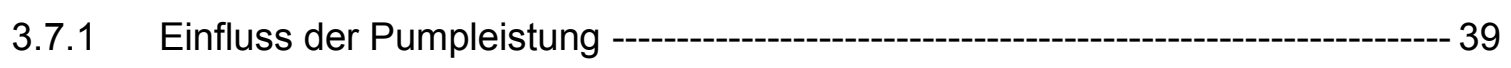

3.7.2 Temperaturabhängigkeit der generischen Kurven ------------------------ 43

3.7.3 Untersuchung der Sputterleistung mittels generischer Kurven-------------------- 45

3.7.4 Einfluss des Argon-Partialdrucks auf die generischen Kurven -------------------- 45

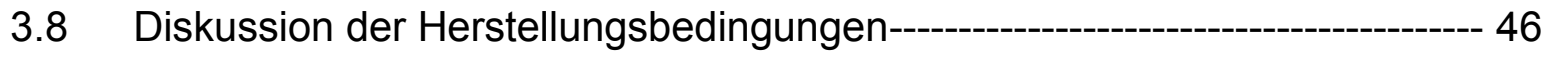

\section{PHYSIKALISCHE EIGENSCHAFTEN DER IRO ${ }_{x}$ SCHICHTEN ------ 48}

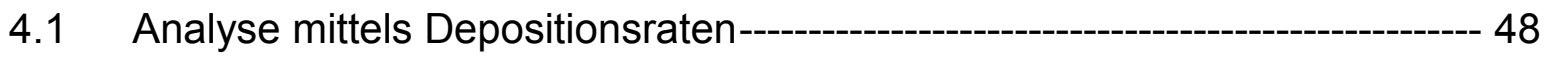

4.2 Analyse der chemischen Zusammensetzung mittels Rutherford Rückstreu-

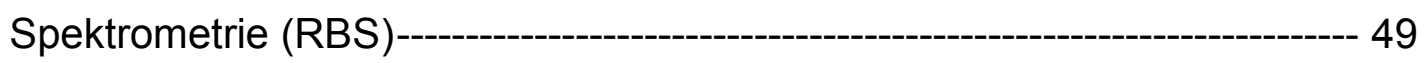

4.3 Charakterisierung der abgeschiedenen $\quad$ Schichten mittels

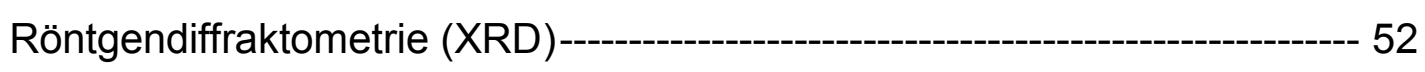

4.4 Schichtcharakterisierung mittels Röntgenreflektometrie (XRR) --------------- 61 
4.5 Analyse der mechanischen Spannungen und Leitfähigkeitsmessungen --- 64

4.6 Oberflächenmorphologieanalysen mittels Rastertunnelmikroskopie (STM) 66

4.7 Transmissionselektronenmikroskopie-Analysen (TEM) ----------------------- 72

\section{REKRISTALLISATIONSVERHALTEN DER IRO ${ }_{x}$ SCHICHTEN------ 76}

$5.1 \quad$ Rasterelektronenmikroskopische Analysen ------------------------------- 76

5.2 In-situ Hochtemperatur Diffraktometrieanalysen (HTXRD)-------------------- 77

5.3 Analyse des elektrischen Widerstandes nach kumulativen

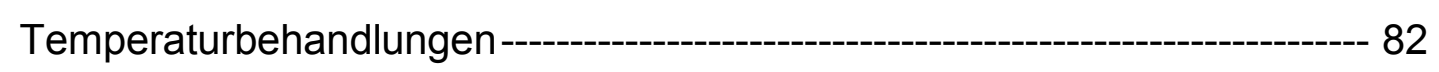

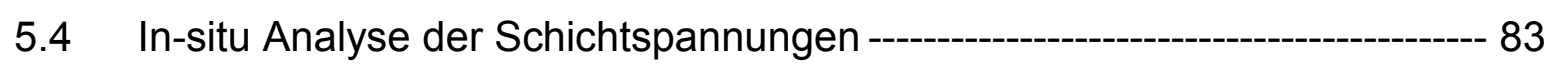

5.5 Differentielle Thermische Analyse--o-

$5.6 \quad$ STM-Analysen der $\mathrm{IrO}_{2}$ Schichten nach thermischer Behandlung ----------- 87

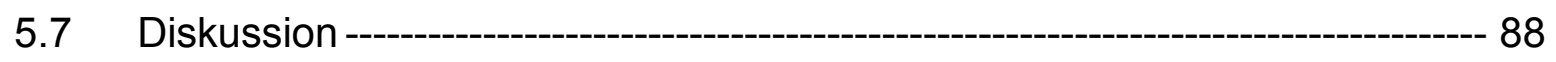

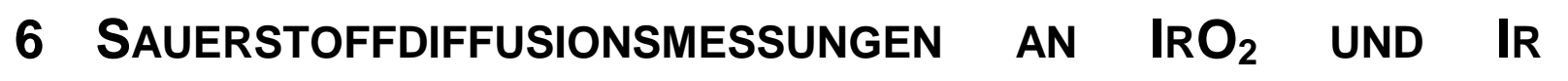

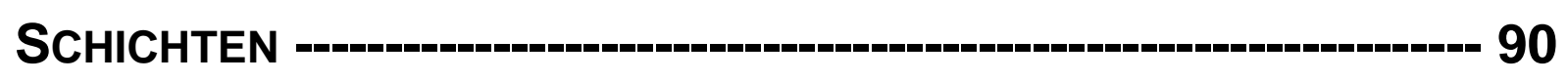

6.1 Verwendeter Diffusionsaufbau --o- 90

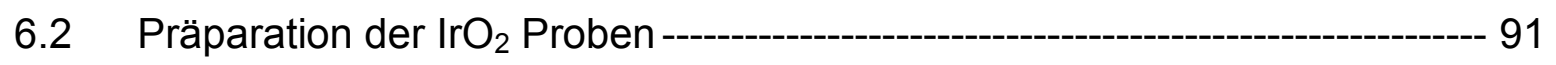

6.3 Messung und Auswertung der ${ }^{18} \mathrm{O}_{2}$ Isotopenprofile ------------------- 92

$6.4 \quad$ Diffusionsmessungen an nicht vorgeglühten $\mathrm{IrO}_{2}$ Proben ------------ 98

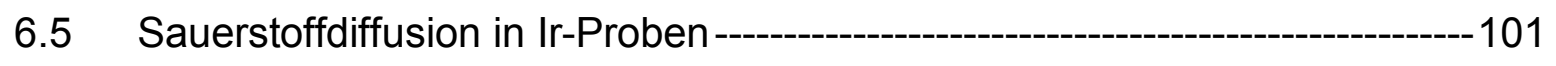

7 INTEGRATION DER IRO 2 SAUERSTOFFBARRIERE --------------105

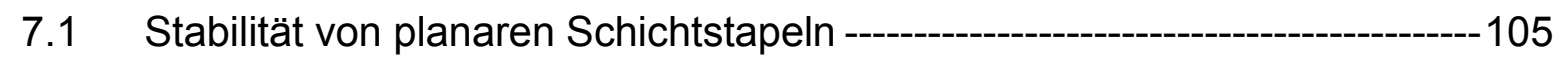




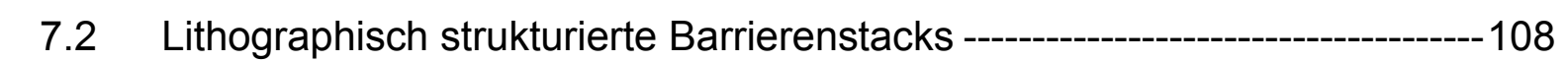

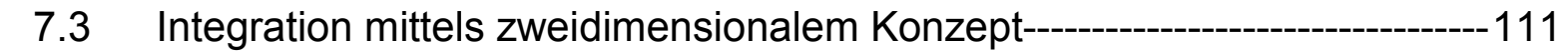

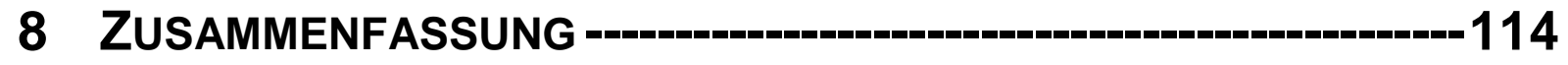

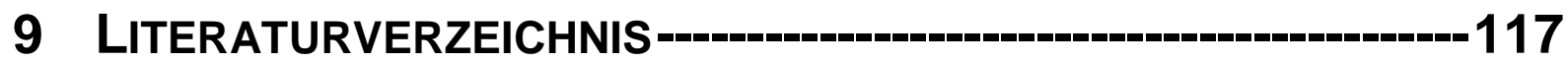




\section{Einleitung}

\subsection{Ziel der Arbeit}

Ziel der Arbeit ist es, im Rahmen der Integration der ferroelektrischen Materialien $\mathrm{SrBi}_{2} \mathrm{Ta}_{2} \mathrm{O}_{9}$ (SBT) und $\mathrm{PbZr}_{\mathrm{x}} \mathrm{Ti}_{1-\mathrm{x}} \mathrm{O}_{3}$ (PZT) in einen Si-Fertigungsprozess mit minimaler Strukturgröße von $0,6 \mu \mathrm{m}$, einen $\mathrm{IrO}_{2}$-Sauerstoffdiffusionsbarrieren-Abscheideprozess zu optimieren und die physikalischen Schichteigenschaften zu charakterisieren. Die Optimierung und Charakterisierung umfasst das Verständnis und die Modellbildung der $\mathrm{IrO}_{\mathrm{x}}$-Abscheidung mittels reaktiver Sputterdeposition in Abhängigkeit der für die Deposition verfügbaren Abscheideparameter. Darüber hinaus soll der Einfluss der Depositionsparameter auf die physikalischen Schichteigenschaften, insbesondere die Sauerstoffselbstdiffusion $\mathrm{im} \mathrm{IrO}_{2}$, geklärt werden. Aus diesen Resultaten sollen die notwendigen Voraussetzungen für eine erfolgreiche Integration des Iridiumdioxids $\left(\mathrm{IrO}_{2}\right)$ als wirksame Sauerstoffbarriere diskutiert, bzw. das mögliche Prozessfenster 1 aufgrund der physikalischen Schichteigenschaften abgesteckt werden.

\subsection{Motivation}

Das Interesse an mobilen und zugleich kompakten elektronischen Anwendungen wie mobiIen Telefonen, IC-Karten und tragbaren Computern ist in den letzten Jahren geradezu explodiert. Aufgrund des großen Marktpotentials hat die Erforschung und Entwicklung von leistungsfähigeren, miniaturisierten statischen und dynamischen Speicherzellen enorm zugenommen. Allein mit einer Minimierung der lateralen Dimensionen stößt diese Weiterentwicklung jedoch an ihre Grenzen, es wird vielmehr nötig, neue Typen von Speicherzellen zu entwickeln, die die Vorteile von kleiner Zellgröße, schnellem dynamischen Zugriff, geringem Leistungsverbrauch und remanenter Informationsspeicherung vereinen. Zu dieser Gruppe von universellen Speicherzellen sind die neuartigen ferroelektrischen (FeRAM ${ }^{2}$ ) und magnetischen (MRAM) Speicherzellen zu zählen. Als Vergleich sind in Tab. 1.1 typische Kenngrößen verschiedener Speicherkonzepte zusammengefasst. Anhand der Daten wird deutlich, dass die ferroelektrische Speicherzelle die Vorteile des extrem schnellen Zugriffs, der geringen Größe und der niedrigen Betriebsspannung der ebenfalls aufgeführten Speicherkonzepte mit der remanenten Datenspeicherung vereint.

\footnotetext{
${ }^{1}$ Prozessfenster ist hier als das maximal mögliche thermische Budget in Temperatur und Zeit zu verstehen, bei dem die Barrierenwirkung der $\mathrm{IrO}_{2}$-Schichten ohne Degradation bestehen bleibt.

${ }^{2}$ RAM, Abkürzung für Random Access Memory (engl.): Speicher mit wahlfreiem Zugriff, d.h. lesbarer und beschreibbarer Speicher, im Unterschied zu ROM, Abkürzung für Read Only Memory (engl.): Speicher mit ausschließlichem Lesezugriff
} 
Somit besitzt dieses Konzept ein hohes Potential, als universeller Speicher eingesetzt zu werden.

Tab. 1.1: Spezifikationen verschiedener Speichertypen nach [Deh99], [Böt01], [Sie99]

\begin{tabular}{lllllll}
\hline & FeRAM & DRAM & SRAM & EEPROM & Flash & MRAM \\
\hline Lesezyklen & $10^{12}-10^{15}$ & $>10^{15}$ & $>10^{15}$ & $>10^{15}$ & $>10^{15}$ & $>10^{15}$ \\
Schreibzyklen & $10^{12}-10^{15}$ & $>10^{15}$ & $>10^{15}$ & $10^{4}-10^{6}$ & $10^{5}-10^{6}$ & $>10^{15}$ \\
Schreibspannung & $0,9-3,3 \mathrm{~V}$ & $0,9-3,3 \mathrm{~V}$ & $0,9-3,3 \mathrm{~V}$ & $12-18 \mathrm{~V}$ & $12-18 \mathrm{~V}$ & $\mathrm{a}$ \\
Schreibzeit & $\mathrm{ns}$ & $\mathrm{ns}$ & $\mathrm{ns}$ & $1-10 \mathrm{~ms}$ & $1 \mu \mathrm{s}-1 \mathrm{~ms}$ & $\mathrm{~ns}$ \\
Zugriffszeit & $40-70 \mathrm{~ns}$ & $40-70 \mathrm{~ns}$ & $6-70 \mathrm{~ns}$ & $40-70 \mathrm{~ns}$ & $40-70 \mathrm{~ns}$ & $40-70 \mathrm{~ns}$ \\
Zellgröße & $\left.8-13 \mathrm{f}^{2} \mathrm{~b}\right)$ & $8 \mathrm{f}^{2}$ & $100 \mathrm{f}^{2}$ & $40 \mathrm{f}^{2}$ & $6-12 \mathrm{f}^{2}$ & $4 \mathrm{f}^{2}$ \\
Datenspeicherung & $>10 \mathrm{Jahre}$ & Keine & Keine & $>10 \mathrm{Jahre}$ & $>10 \mathrm{Jahre}$ & $>10 \mathrm{Jahre}$ \\
\hline
\end{tabular}

\footnotetext{
a) bei MRAMs sind die Schreibströme relevant

b) $f(=$ minimum feature size): die kleinste in der jeweiligen Technologie verwendete Strukturgröße
}

Die Idee, die hinter dem Einsatz von elektrisch remanent polarisierbaren Dielektrika steht, ist schon in den 50er Jahren entstanden [Auc98], scheiterte aber bislang an der Integration der dafür benötigten Materialien in die auf Silizium basierende Halbleiterumgebung. Prinzipiell werden derzeit drei Realisierungsmöglichkeiten der Integration von ferroelektrischen Materialien verfolgt, die sich an die in Abb. 1.1 a) gezeigte DRAM Zelle anlehnen. Die DRAM Zelle besteht aus einem Auswahltransistor $T$ und einem Kondensator $C$, der für die Speicherung der Zellinformation dient.
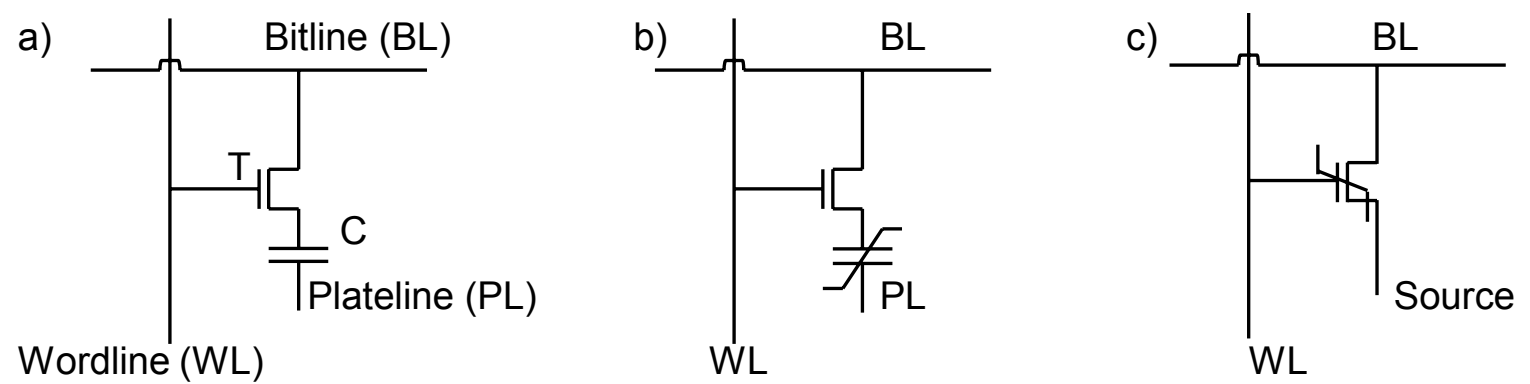

Abb. 1.1: Zellkonzepte nach [Koh01], a) DRAM Zelle, b) 1T-1C-FeRAM Zelle, c) 1T-FeFET Zelle

Die ferroelektrische 2T-2C Zelle, die in Abb. 1.1 b) gezeigte 1T-1C Zelle und die in Abb. 1.1 c) abgebildete 1T Zelle verdeutlichen die prinzipielle Ähnlichkeit zum DRAM-Zellkonzept [Auc98]. Bei der 2T-2C Zelle wirkt ein zusätzlicher Transistor und ein Kondensator als Refe- 
renz für den Speicherkondensator [Eva88], [Sco00], dies garantiert eine höhere Auslesesicherheit, erhöht aber im Vergleich zur 1T-1C Zelle die Zellgröße. Bei beiden Zellkonzepten ist das Kondensatordielektrikum durch ein ferroelektrisches Material ersetzt. Der Schreibvorgang wird durch Anlegen eines elektrischen Feldes erreicht, was eine dauerhafte Polarisation des Ferroelektrikums bewirkt. Der Lesevorgang wird in den beiden zuerst genannten Zellkonzepten durch Überschreiben der Zellen und anschließendes Auswerten des StromzeitIntegrals bestimmt, das abhängig von der in der Zelle zuvor gespeicherten Bitinformation ist [Böt01]. Man bezeichnet dies als destruktiven Ausleseprozess, denn anschließend muss die überschriebene Information wieder zurückgeschrieben werden. Trotz des aufwändigen Ausleseschritts ist die 1T-1C Zelle für hohe Integrationsdichten zur Zeit das vielversprechendste Konzept. Bei der 1T Zellkonfiguration, die man auch als Ferro-FET (Feld-Effekt-Transistor) bezeichnet, wird eine dünne ferroelektrische Schicht anstelle eines Gate-Oxids unterhalb des Transistor-Gate-Anschlusses eines FET abgeschieden, über die Polarisation wird die Ladungsträgerkonzentration im Kanal gesteuert [Mil92].

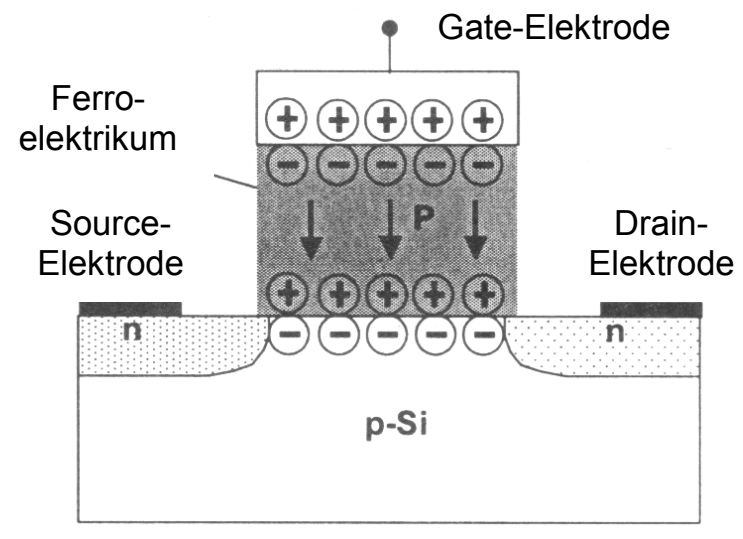

Abb. 1.2: 1T Zelle im leitenden Inversionszustand, schematisch, nach [Koh01]

Dieses Zelldesign hat im Vergleich zu den anderen zwei genannten Zellarchitekturen den Vorteil, dass der Lesevorgang nicht-destruktiv ist, da nur der Kanalwiderstand in Abhängigkeit des Polarisationszustandes bestimmt werden muss. Es ist jedoch bisher noch nicht gelungen, einen Ferro-FET herzustellen, der eine für Anwendungen ausreichend lange Informationsspeicherung sicherstellt. Der Grund dafür ist eine während der Herstellung oder der notwendigen thermischen Nachbehandlung entstehende Grenzflächendegradation, die eine Ladungsträgerinjektion aufgrund von raumfesten Ladungen bewirkt [Koh01]. Im folgenden wird jedoch nur die Integration der 1T-1C Zelle näher untersucht, da dieses Zellkonzept das größte Potential hat, um in den Mbit und Gbit Speicherbereich vorzustoßen [Auc98]. 


\subsection{Ferroelektrische Materialien}

Ferroelektrika gehören zur Klasse der pyroelektrischen Kristalle, deren spontane elektrische Polarisation sich durch das Anlegen eines äußeren Feldes umkehren lassen kann. Die Polarisation $P$ eines Ferroelektrikums weist im äußeren elektrischen Feld E ein Hystereseverhalten auf, welches schematisch in Abb. 1.3 gezeigt ist. Ein ferroelektrisches Material ist durch das Auftreten einer remanenten elektrischen Polarisation $P_{r}$ charakterisiert, da der ferroelektrische Zustand im Gegensatz zu dem eines Elektreten ${ }^{3}$ ein thermischer Gleichgewichtszustand ist [Sco00]. Oberhalb einer materialspezifischen kritischen Übergangstemperatur $T_{C}$ verschwindet die Ferroelektrizität, das Material befindet sich dann im paraelektrischen Zustand. Amorphe Materialien können aufgrund der nicht vorhandenen Kristallsymmetrie nicht ferroelektrisch sein.

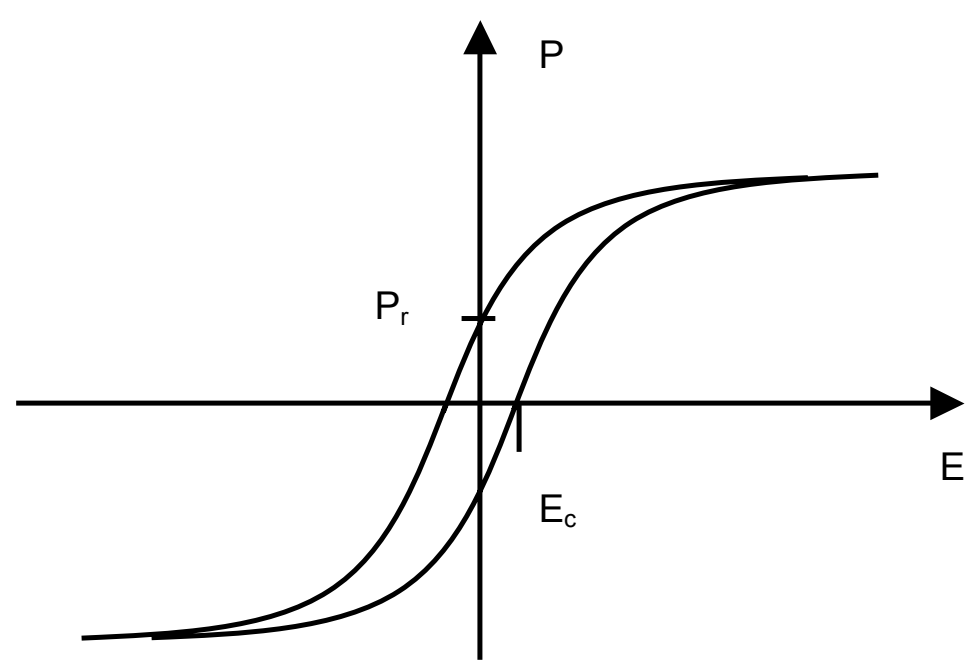

Abb. 1.3: Hystereseschleife in der Polarisation $P$ eines Ferroelektrikums im äußeren elektrischen Feld $\mathrm{E}, \mathrm{P}_{\mathrm{R}}$ : remanente Polarisation, $\mathrm{E}_{\mathrm{C}}$ : Koerzitivfeldstärke

Die ferroelektrische Eigenschaft der remanenten ionischen Polarisation beruht darauf, dass sich unterhalb der Curie-Temperatur eine bistabile W-förmige Potentialmulde für das zentrale Atom in der Perowskit-Einheitszelle ausbildet, welche schematisch in Abb. 1.4 b) gezeigt ist. Wenn dieses Potential $\mathrm{U}(\mathrm{x})$ temperaturunabhängig ist, dann spricht man bei zunehmender Temperatur von einem Ordnungs-Unordnungs-Übergang, da die kinetische Energie $\mathrm{k}_{\mathrm{B}} \mathrm{T}$ des Zentralions groß genug wird, die Potentialbarriere zu überspringen, so dass die zeitlich gemittelte Position $<x>$ des lons in der Mitte der zwei Potentialmulden liegt. Der Fall des temperaturabhängigen Potentials $\mathrm{U}(\mathrm{x}, \mathrm{T})$, also das Auftreten eines displaziven Übergangs wird erfolgreich durch die Landau-Devonshire Theorie beschrieben [Lan37], [Dev49]. Diese geht

\footnotetext{
${ }^{3}$ Ein Elektret zeigt im Gegensatz zu einem Ferroelektrikum eine Relaxation der Polarisation nach dem
} Abschalten des externen elektrischen Feldes 
davon aus, dass die Freie Energie $\mathrm{F}$ in geraden Potenzen der Polarisation $\mathrm{P}$ mit den Koeffizienten $A$, B und $C$ gemäß

$$
F(P, T, E)=-E P+c+A P^{2}+B P^{4}+C P^{6}
$$

entwickelt werden kann. Dabei stellt $E$ das elektrische Feld und c eine Konstante dar, der Koeffizient $A$ des quadratischen Terms ist gegeben durch

$$
A=A_{0}\left(T-T_{0}\right)
$$

Hier ist $\mathrm{T}_{0}$ eine Temperatur, die bei einem Übergang erster Ordnung kleiner als die Curie Temperatur ist $\left(T_{0}<T_{C}\right)$, bei einem Übergang zweiter Ordnung mit der Curie-Temperatur zusammenfällt $\left(\mathrm{T}_{0}=\mathrm{T}_{\mathrm{C}}\right)$. Ausführliche Darstellungen zur Ferroelektrizität werden beispielsweise von Scott [Sco00], Kittel [Kit96] und Jona [Jon62] gegeben.

a)

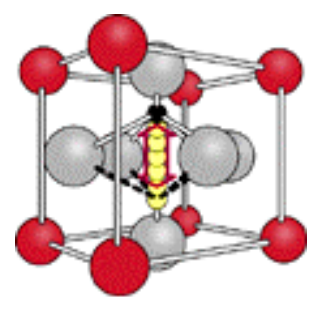

b)

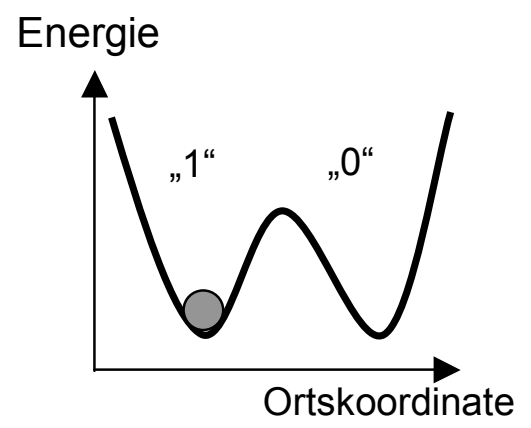

Abb. 1.4: Schematische Einheitszelle des $\mathrm{PZT}$ (rot: $\mathrm{Pb}^{2+}$, grau: $\mathrm{O}^{2-}$, schwarz: $\mathrm{Zr}^{4+}$ oder $\mathrm{Ti}^{4+}$ ), WPotential nach [Ram01], b) schematischer Potentialverlauf für das zentrale lon

Für den kommerziellen Einsatz in FeRAM-Zellen haben sich perowskitische oder perowskitähnliche quaternäre Oxidschichten als geeignet erwiesen, darunter das Blei-Zirkon-Titanat $\mathrm{PbZr}_{\mathrm{x}} \mathrm{Ti}_{1-\mathrm{x}} \mathrm{O}_{3}$ (PZT) und das Strontium-Bismut-Tantaloxid $\mathrm{SrBi}_{2} \mathrm{Ta}_{2} \mathrm{O}_{9}$ (SBT) [Sco00]. Das PZT hat, in der Zusammensetzung $\mathrm{PbZr}_{0,55} \mathrm{Ti}_{0,45} \mathrm{O}_{3}$, eine Curie Temperatur von $\mathrm{T}_{\mathrm{C}}=340^{\circ} \mathrm{C}$, für das SBT werden ähnliche Werte von $310<\mathrm{T}_{\mathrm{C}}<335^{\circ} \mathrm{C}$ angegeben [Gal69], [Smo61], [Sub62]. Die beiden genannten Materialien erfüllen die maßgeblichen ferroelektrischen Eigenschaften, die für die erfolgreiche Integration vorauszusetzen sind. Dazu gehören a) eine geringe Koerzitivfeldstärke, die für den Betrieb der Speicherzellen mit geringer Arbeitsspannung notwendig ist, b) eine ausreichend hohe remanente Polarisation, die das korrekte Auslesen der zuvor geschriebenen Bit-Information sicherstellt und c) keine signifikante Reduktion dieser remanenten Polarisation durch wiederholtes zyklisches Auslesen und Schreiben der Zellen. Dieses Phänomen bezeichnet man als Ermüdung, das nach aktuellem Forschungsstand einer reduzierten Domänenwandmobilität und der Elektroden-Ferroelektrikum Grenzflächendegra- 
dation durch Sauerstoffleerstellen-Agglomeration zugeschrieben wird [Lee95]. Beide Effekte bewirken eine Erniedrigung der Polarisation der ferroelektrischen Schicht.

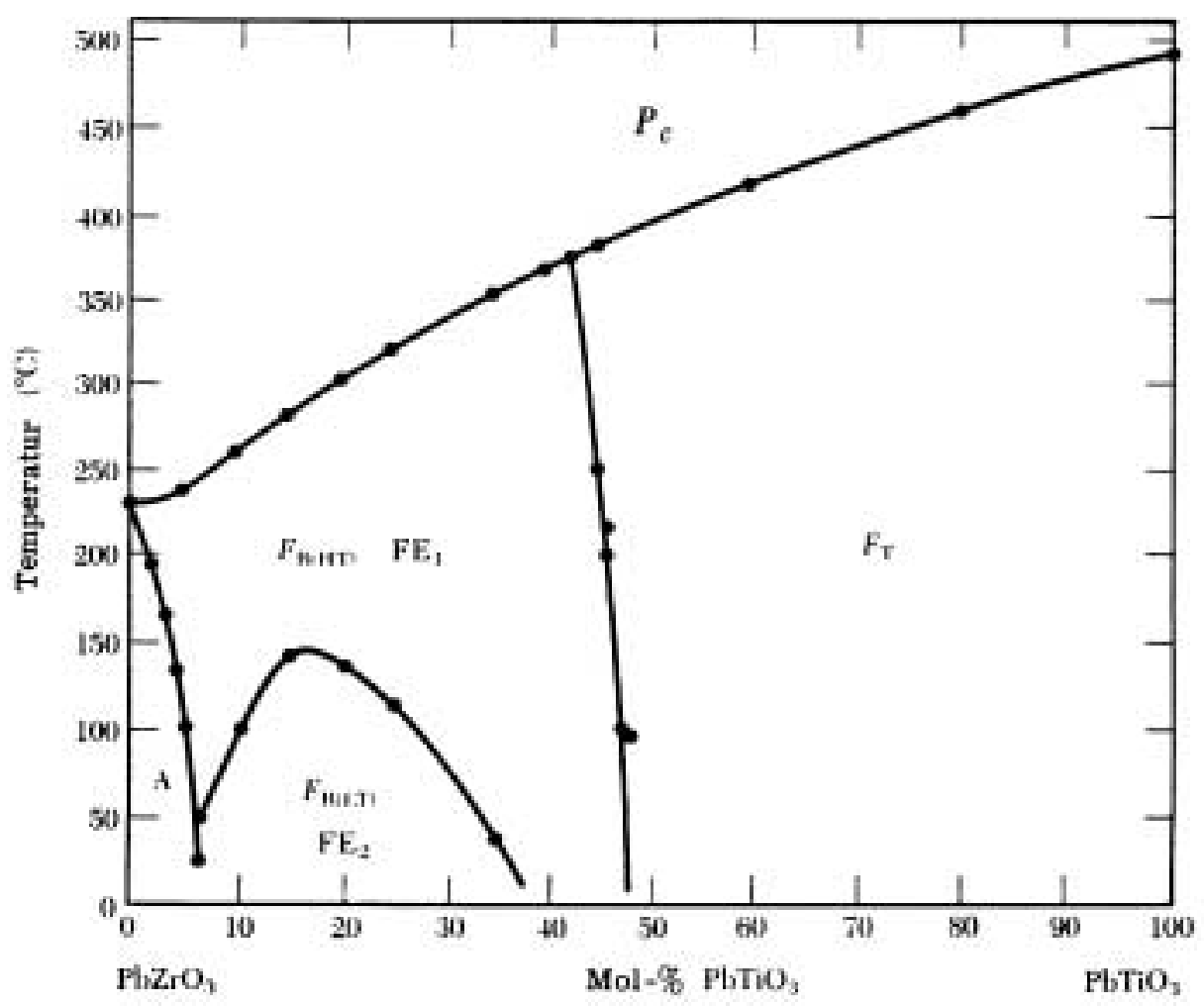

Abb. 1.5: Phasendiagramm des Systems $\mathrm{PbZrO}_{3}-\mathrm{PbTiO}_{3}$ aus [Jaf71], $\mathrm{P}$ : paraelektrisch, $\mathrm{F}$ : ferroelektrisch, A: antiferroelektrisch, R: rhomboedrisch, T: tetragonal, C: kubisch, HT: Hochtemperaturphase, LT: Tieftemperaturphase

Das in Abb. 1.5 gezeigte Phasendiagramm des $\mathrm{PbZr}_{x} \mathrm{Ti}_{1-x} \mathrm{O}_{3}$ (PZT) weist neben der Existenz verschiedener ferroelektrischer Phasen zusätzlich bei sehr geringen Ti-Konzentrationen eine antiferroelektrischen Phase auf [Kit96]. Für hohe Zr/Ti-Verhältnisse bildet sich eine rhomboedrische Phase, von der es eine Hochtemperatur- und eine Tieftemperaturform gibt. Rechts der in Abb. 1.5 gezeigten nahezu temperaturunabhängigen morphotropen Phasengrenze existiert ausschließlich die tetragonale ferroelektrische Phase. Dabei handelt es sich um den in Abb. 1.4 a) gezeigten perowskitischen Gittertyp mit einer leicht tetragonalen Verzerrung, bei der die c-Achse eine typische Dilatation im Promill- bis Prozentbereich aufweist [Hoo01], [Jon62]. Es ist weiterhin aus dem Phasendiagramm ersichtlich, dass der Übergang von den ferroelektrischen Phasen in den paraelektrischen Zustand zwar stark abhängig vom $\mathrm{Ti} / \mathrm{Zr}$ Verhältnis des Materials ist, jedoch weit oberhalb von Temperaturen liegt, die für die Speicheranwendungen relevant sind. Durch Variation des Ti/Zr-Verhältnisses der Schichten ist es möglich, die bereits diskutierten ferroelektrischen Eigenschaften zu optimieren [Aok98], [Sak99]. 
Um die Bildung einer Perowskit-Phase in den beschriebenen quaternären Materialien zu erreichen, sind, ähnlich wie bei der Herstellung von $\mathrm{YBa}_{2} \mathrm{Cu}_{3} \mathrm{O}_{7^{-}}$Hochtemperatursupraleitern oder magnetischen $\mathrm{La}(\mathrm{Ca}, \mathrm{Sr}) \mathrm{MnO}_{3}$ Schichten, hohe Anlass- oder Depositions-Temperaturen von typischerweise $550^{\circ}-750^{\circ} \mathrm{C}$ notwendig [Luo01], [Nog96]. Die perowskitische Phasenbildung wird im allgemeinen durch geeignetes Nachtempern in sauerstoffhaltiger Umgebung nach der Abscheidung (z.B. durch PVD ${ }^{4}$ oder PLD ${ }^{5}$, MOD ${ }^{6}$ oder MOCVD [Moo01]. Bei den PZT und SBT Schichten weist jeweils nur die perowskitische Kristallstruktur ferroelektrische Eigenschaften auf. Die Schichten, die bei zu niedrigen Temperaturen hergestellt werden, wachsen amorph auf und besitzen daher keine ferroelektrischen Eigenschaften. Nur durch das anschließende Anlassen dieser amorphen Schichten bei geeignet hohen Temperaturen ist es möglich, die Kristallisation dieser ferroelektrischen Phase zu erreichen. Bei niedrigeren Anlasstemperaturen bildet sich, abhängig von Material und thermischer Behandlung, eine dielektrische Fluorit- oder eine Pyrochlor-Phase aus, die keine ferroelektrischen Eigenschaften besitzt [Li00].

Für die Realisierung des 1T-1C Konzeptes gibt es prinzipiell zwei Möglichkeiten: die StackZelle $^{\text {B }}$ und die Offset-Zelle ${ }^{9}$. Bei der Stack-Zelle wird das ferroelektrische Material direkt über dem elektrischen Drain-Kontakt zum Transistor abgeschieden, das heißt, der Kondensator wird direkt vertikal über dem Transistor formiert. Beim Offset-Konzept wird der Kondensator mit einem lateralen Versatz neben dem vertikalen elektrischen Transistor-Kontakt gebildet. Dieses Konzept hat den Vorteil einer deutlich vereinfachten Integration, da die elektrische Kontaktierung zwischen Transistor und Kondensatorelektrode mit einem sogenannten plug erst nach der Hochtemperaturbehandlung erfolgt. Um jedoch für kommende Generationen eine möglichst hohe Integrationsdichte zu erreichen (4Mbit und deutlich höher) ${ }^{11}$, ist nur das zuerst genannte Stack-Konzept geeignet, da es eine verkleinerte Zellgröße ermöglicht. In Abb. 1.6 sind rasterelektronenmikroskopische Querschnitts-Aufnahmen (REM) der beiden Integrationskonzepte vorgestellt.

\footnotetext{
${ }^{4}$ PVD: Physical Vapor Deposition (Deposition mittels Sputter- oder Aufdampfverfahren)

${ }^{5}$ PLD: Pulsed Laser Deposition (Deposition durch Laserablation)

${ }^{6}$ MOD: Metal Organic Deposition (Deposition über Schleuder- oder Sprayverfahren)

${ }^{7}$ MOCVD: Metal Organic Chemical Vapor Deposition (Chemische Deposition aus der Dampfphase)

${ }^{8}$ stack (engl.): Stapel

${ }^{9}$ offset (engl.): (laterale) Verschiebung, Abzweigung, Nebenleitung

${ }^{10}$ plug (engl.): Stöpsel, Stecker

${ }^{11} 4$ Mbit bedeutet 4 Millionen bit (für 1T-1C Zellen 4 Mio. FE-Kondensatoren) pro Quadratzoll, $1 \mathrm{Zoll}=2,54 \mathrm{~cm}$
} 

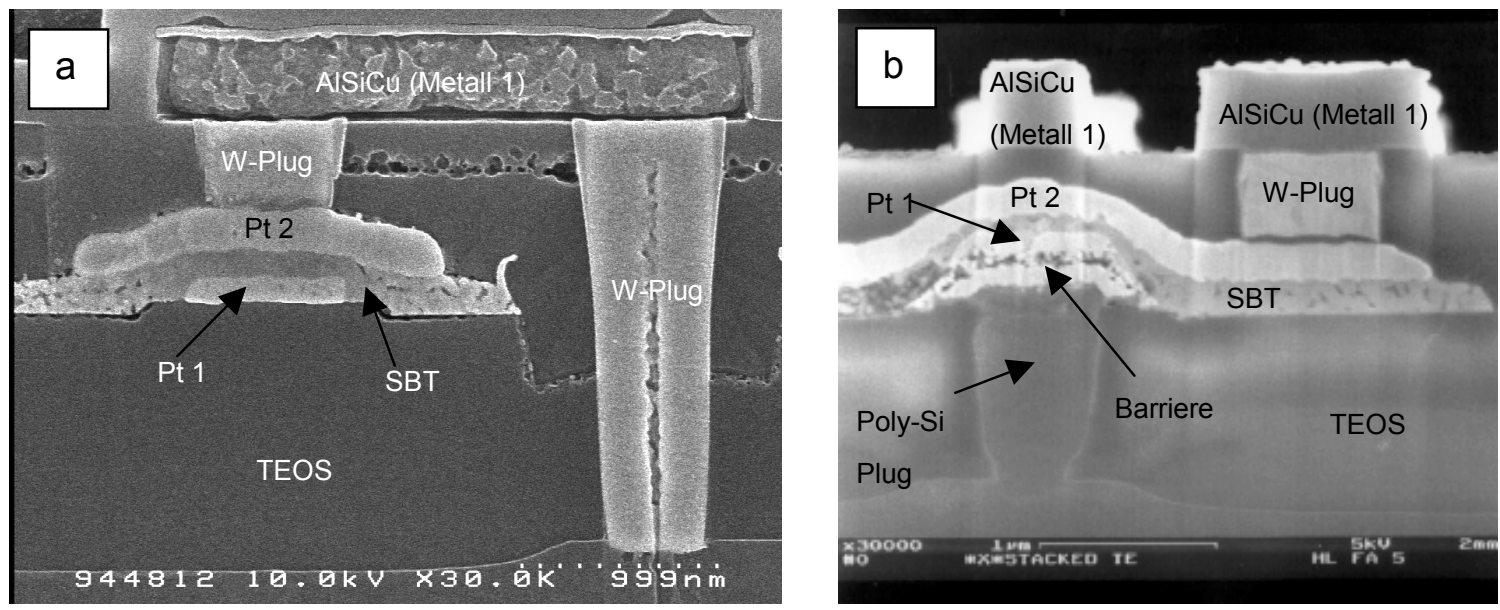

Abb. 1.6: REM Bilder einer (a) Offset- und (b) Stack-Zelle zur Realisierung einer 4M 1T-1C FeRAMZelle in 0,6 $\mu \mathrm{m}$-Technologie [Deh99], [Nag00]

Auf den REM-Aufnahmen sind jeweils der Pt/SBT/Pt Kondensator, sowie die unterschiedliche elektrische Anbindung an den Transistor gezeigt. Dabei werden Pt-Elektroden für den SBT-Kondensator verwendet, da sie keine Oxidbildung bei Hochtemperaturbehandlungen in Sauerstoffumgebung zeigen und besonders gute elektrische Eigenschaften des SBTKondensators sicherstellen: Pt/SBT/Pt Kondensatoren weisen eine für NiedrigspannungsAnwendungen notwendige geringe Koerzitivfeldstärke auf und zeigen eine geringe ferroelektrische Ermüdung [Ara95], [Auc98]. Beim Stack-Konzept (b) wird der Kondensator direkt über einen Si-plug mit dem Transistor verbunden, beim Offset-Konzept (a) wird zunächst der Kondensator geformt, anschließend werden mittels Wolframfüllung die tiefen Kontakte zum Transistor gebildet. Der Transistor und der Kondensator sind durch ein etwa $1 \mu \mathrm{m}$ dickes TEOS 12 Dielektrikum voneinander getrennt.

Die Integration einer ferroelektrischen Schicht in eine Siliziumumgebung setzt jedoch voraus, die Diffusion des in der Prozessatmosphäre und im Ferroelektrikum vorhandenen Sauerstoffs sowie der anderen atomaren Bestandteile des Ferroelektrikums zu unterbinden. Weiterhin muss eine die Kondensatorstruktur zerstörende chemische Reaktion mit den Elektroden vermieden werden. Die Ausdiffusion der für die gewöhnliche Halbleiterumgebung neuartigen Elemente wie $\mathrm{Bi}, \mathrm{Sr}$ oder $\mathrm{Pb}$, deren Langzeiteinfluss auf die elektronischen CMOSSchaltkreise noch nicht hinreichend bekannt ist, muss ebenfalls verhindert werden. Die Integration des Ferroelektrikums mittels Barriereschichten ist in dem in Abb. 1.7 gezeigten Flussdiagramm schematisch dargestellt.

\footnotetext{
${ }^{12}$ TEOS: pyrolytisch aus Tetraethylorthosilikat abgeschiedenes $\mathrm{SiO}_{2}$
} 


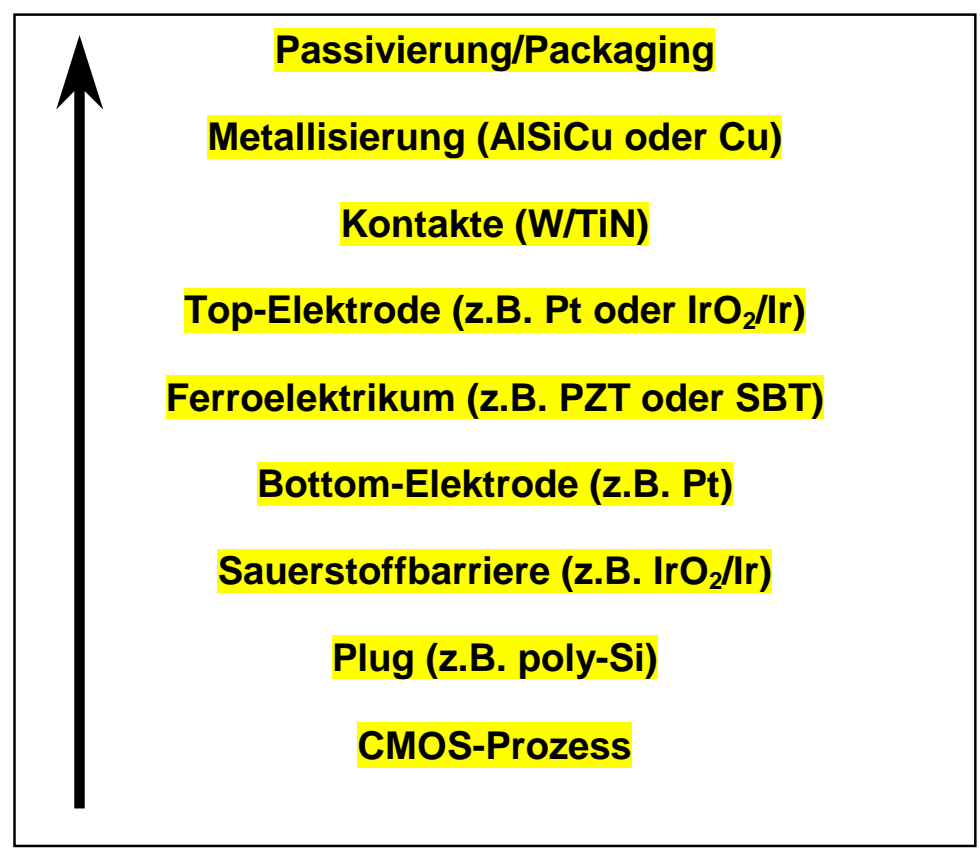

Abb. 1.7: Flussdiagramm zur Integration des Ferroelektrikums, der Pfeil deutet die zeitliche Abfolge der Prozesse an.

Die Konsequenz einer Sauerstoffdiffusion in die aus dotiertem polykristallinen Silizium bestehende elektrische Verbindung, die das Draingebiet des Transistors mit dem Kondensator kontaktiert, wäre eine Oxidation des Siliziums. Durch den daraus resultierenden Verlust des elektrischen Kontaktes ist die Speicherzelle nicht mehr ansteuerbar. Um dieses Problem zu überwinden, werden Diffusionsbarrieren eingesetzt, also Schichten, die sich durch eine hohe thermische Stabilität auszeichnen, chemisch inert sind und keine nennenswerte Eindiffusion zulassen. Weitere wichtige Aspekte für die Wahl der Diffusionsbarriere ist die Kontrollierbarkeit der mechanischen Spannungen und eine gute Haftung der Schichten auf dem Substratmaterial. Das für diesen Zweck in Frage kommende Material soll weiterhin gut elektrisch leitfähig sein, aus produktionstechnischer Sicht sollte es zudem höchst reproduzierbar und kostengünstig abzuscheiden sein.

Die Wahl der für die Integration nutzbaren Materialien ist aufgrund der extrem hohen und zahlreichen Anforderungen sehr beschränkt. Für diese Zwecke sind in der Mikroelektronik häufig nitrid-basierte amorphe und (nano-)kristalline Materialien wie TiN [Bei99] oder ternäre Barrierenschichten wie Ta-Si-N [Cab00], Ti-Si-N [Kac99], Ti-Al-N [Mcl90], [Kim01], oder Ir-Si$\mathrm{N}$ [Mat00] entwickelt und eingesetzt worden. Diese Materialien zeigen bei den für die Integration der oben genannten ferroelektrischen Materialien erforderlichen Prozessbedingungen jedoch nur bedingte, zeitlich limitierte Oxidationsbeständigkeit, da die genannten Nitride keine thermodynamische Langzeitstabilität in Kontakt mit Sauerstoff besitzen [Har97]. Die Konsequenz ist eine Degradation der elektrischen Kondensatoreigenschaften bzw. sogar der 
Verlust der elektrischen Leitfähigkeit der Nitridbarriere durch die Bildung einer dünnen Oxidschicht [Kim01].

Als Materialien, die den zuvor genannten Ansprüchen genügen und in Kontakt mit Sauerstoff bei den typischen Prozesstemperaturen thermodynamisch stabil sind, kommen leitfähige metallische Oxide in Betracht, wie z.B. die Perowskite $\mathrm{LaSrCoO}_{3}$ [Nag99], $\mathrm{SrRuO}_{3}$ [Lin00], die binären Oxide $\mathrm{IrO}_{2}$ [Cho97] oder $\mathrm{RuO}_{2}$ [Oh00], oder ternäre, teils amorphe Ta-X-O [Yoo99], [Zha99] und X-Si-O Schichten (mit X=Ir, Ru, Rh) [Gas99]. Dabei sind die binären Oxide der Pt-Metalle Ru und Ir aufgrund der geringeren Komplexität im Vergleich zu ternären oder quaternären Schichten aus Integrationsaspekten und aus Gründen der Reproduzierbarkeit vorzuziehen. Zudem wurde nachgewiesen, dass ternäre amorphe Oxide der letztgenannten Art nicht die notwendige thermische Stabilität in Sauerstoffatmosphäre aufweisen [Gas99a]. Nakamura et al. [Nak94] haben gezeigt, dass das $\mathrm{IrO}_{2}$ aufgrund der guten elektrischen Leitfähigkeit von unter $50 \mu \Omega \mathrm{cm}$ [Gme78] auch gleichzeitig die Rolle der Elektrode und der Sauerstoff-Barriere übernehmen kann. Dies führt bei PZT-Kondensatoren sogar zu einer deutlichen Verbesserung der elektrischen Eigenschaften, da die ferroelektrische Ermüdung dadurch deutlich reduziert werden kann.

In dieser Arbeit wird aus den zuvor diskutierten Gründen die Integration des Barrierensystems Ir/lrO ${ }_{2}$ verfolgt, da das Ru-O System neben dem festen $\mathrm{RuO}_{2}$ ein schon bei RT flüchtiges, brennbares, giftiges Tetraoxid $\mathrm{RuO}_{4}$ besitzt [Bal99], eine Eigenschaft, die aus thermodynamischer Sicht schon a priori ungünstig erscheint. Ir hingegen bildet im relevanten Temperaturbereich nur ein festes Oxid, das in der Rutilstruktur ${ }^{13}$ kristallisierende $\mathrm{IrO}_{2}$; dieses beginnt erst bei Temperaturen von über $1000^{\circ} \mathrm{C}$ als nicht giftiges gasförmiges $\mathrm{IrO}_{3}$ zu sublimieren [Gme78].

\subsection{Das System Ir-O}

Iridiumdioxid $\left(\mathrm{IO}_{2}\right)$ gehört zu den Übergangsmetalloxiden, die ein bemerkenswert breites Spektrum an strukturellen, elektrischen und magnetischen Eigenschaften aufweisen, welche der Existenz der äußeren d-Elektronen der Übergangsmetallionen zugeschrieben wird. Die Lage des höchsten Metall s-Bandes sowie die des Sauerstoff p-Bandes wird in den Oxiden energetisch stark angehoben bzw. abgesenkt, so dass das d-Band als einziges in der Nähe der Fermienergie liegt und damit für die elektrischen Eigenschaften der Oxide verantwortlich ist [Adl68].

\footnotetext{
${ }^{13}$ Wegen der Isomorphie zum $\mathrm{TiO}_{2}$ wird die Kristallstruktur des $\mathrm{IrO}_{2}$ auch als Rutil bezeichnet, rutilus (lat.)=rötlich
} 
Die unterschiedliche Ausprägung der Coulomb-Abschirmung benachbarter Atome kann nach Mott [Mot61] zu itinerantem Verhalten, also metallischer Leitfähigkeit, aber auch zur Lokalisation der Elektronen, also isolierendem Verhalten führen [Goo71]. Die Übergangsmetalloxide $\mathrm{IrO}_{2}, \mathrm{RuO}_{2}, \mathrm{RhO}_{2}$ und $\mathrm{OsO}_{2}$ zeichnen sich durch eine gute metallische Leitfähigkeit aus, $\mathrm{IrO}_{2}$ zeigt einen an Einkristallen gemessenen anisotropen spezifischen Widerstand von $\rho_{a}=27 \mu \Omega \mathrm{cm}$ und $\rho_{c}=49 \mu \Omega \mathrm{cm}$ entlang der a- bzw. der c-Achse.

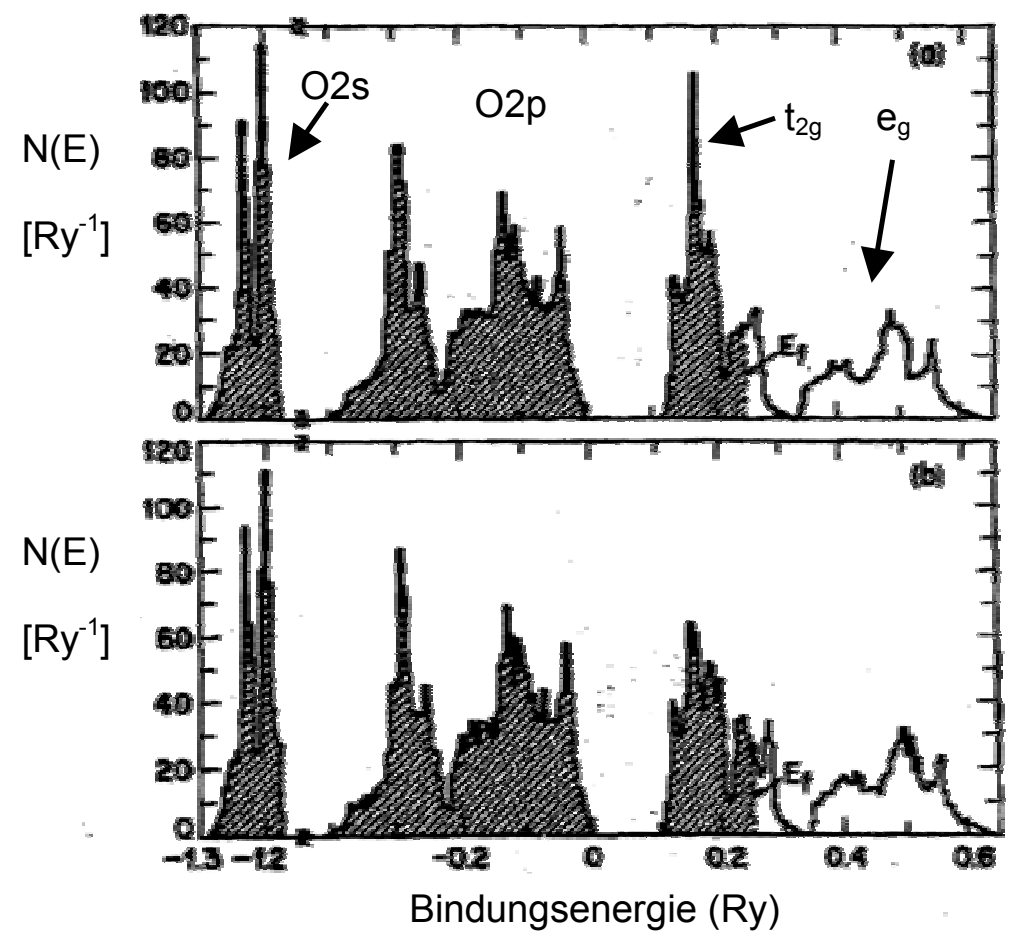

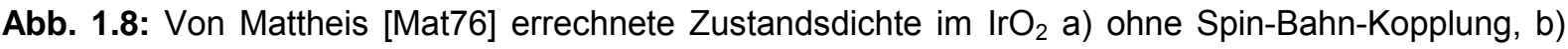
mit Spin-Bahn-Kopplung. Auf der Ordinate sind die Anzahl der Zustände pro Einheitszelle aufgetragen. $E_{f}$ : Fermie-Energie (1 Ry=13,6 eV)

Damit stellt $\mathrm{IrO}_{2}$ das Oxid mit der besten Leitfähigkeit der zuvor genannten Pt-Metalloxide dar [Rao74]. Für $\mathrm{IrO}_{2}$ existieren Bandstrukturrechnungen [Mat76], [Gra75], [Ryd72], [Ryd70], die in gutem Einklang mit den experimentellen spektroskopischen Ergebnissen stehen [Wer80], [Dan84]. Abb. 1.8 Zeigt die von Mattheis [Mat76] für das $\mathrm{IrO}_{2}$ errechnete Zustandsdichte, die die energetische Lage des Ferminiveaus und der relevanten Bänder zeigt. Das Ferminiveau liegt im $5 d$-Band, das wegen des oktaedrischen Ligandenfeldes in eine $t_{2 g}$ und eine $e_{g}$ Komponente aufspaltet. Die spezifischen Widerstände von $\mathrm{IrO}_{2}$ und Ir sind in Tab. 1.2 zusammen mit den Werten von einigen für die Halbleiter-Technologie relevanten Metallen zusammengefasst. 
Tab. 1.2: Spezifische Widerstände von in der Halbleiter-Technologie eingesetzten Metallen, nach [Mur93]

\begin{tabular}{ll}
\hline Material & $\begin{array}{l}\text { Spezifischer } \\
\text { Widerstand }[\mu \Omega \mathrm{cm}]\end{array}$ \\
\hline $\mathrm{Cu}$ & 1,67 \\
$\mathrm{Al}$ & 2,65 \\
$\mathbf{W}$ & 5,65 \\
$\mathrm{Ti}$ & 42 \\
Ir & 4,7 \\
$\mathrm{IrO}_{2}$ & $27 / 49$ \\
\hline
\end{tabular}

Für das System Ir-O wird aufgrund der sehr geringen Löslichkeit von $\mathrm{O}$ im Ir bzw. im $\mathrm{IrO}_{2}$ in Standardwerken kein Phasendiagramm angegeben [Mas86], [Ell65], [Gme78]. In dem System ist nur die Existenz zweier fester Phasen bekannt: die in kubisch flächenzentrierter Struktur $(a=3,8394 \AA)$ kristallisierende Ir-Phase und die tetragonale $\operatorname{IrO}_{2}$-Phase $(a=4,4983 \AA$, $\mathrm{c}=3,1544 \AA$ ) [JCP95], [Cor62]. In der Rutil-Phase des $\mathrm{IrO}_{2}$ befinden sich die Ir-Atome auf den Eckplätzen der tetragonalen Einheitszelle, ein weiteres Ir-Atom ist in der Mitte der Einheitszelle von Sauerstoffatomen in oktaedrischer Konfiguration umgeben, wie Abb. 1.9 schematisch zeigt. Auf den (200)-Ebenen befinden sich ausschließlich Ir-Atome, die dichtest gepackten Ebenen sind die (110)-Ebenen. Ir ist ein Edelmetall der Pt-Elemente, besitzt nach Os 14 die zweithöchste Dichte $\left(22,6 \mathrm{~g} / \mathrm{cm}^{3}\right)$ des PSE ${ }^{15}$ und ist chemisch sehr inert. Das $\mathrm{IrO}_{2}$ besitzt im Gegensatz zum Ir eine etwa halb so große Dichte von 11,6 g/ $\mathrm{cm}^{3}$. Es wird neben den beiden genannten festen Phasen in der Literatur die Existenz der gasförmigen molekularen $\mathrm{IrO}_{3}$ Phase bestätigt, die bis zu Temperaturen von etwa $1700^{\circ} \mathrm{C}$ stabil ist [Sch60], weiterhin wird das Vorkommen eines metastabilen, ebenfalls gasförmigen Sesquioxids $\operatorname{Ir}_{2} \mathrm{O}_{3}$ diskutiert [Gre88].

\footnotetext{
${ }^{14}$ Os: Osmium

${ }^{15}$ PSE: Periodensystem der Elemente
} 


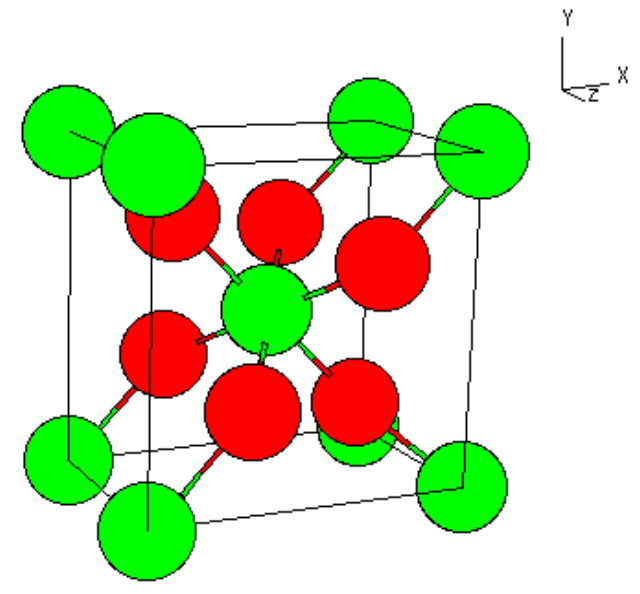

Abb. 1.9: Schematische Darstellung der Rutil-Kristallstruktur des $\mathrm{IrO}_{2}$, grün: Ir-Atome, rot: O-Atome. Die Ir-Atome bilden ein raumzentriertes tetragonales Gitter, die Positionen der Sauerstoffatome sind $r_{1}=(u,-u, 0), r_{2}=(-u, u, 0), r_{3}=(1 / 2-u, 1 / 2-u, 1 / 2), r_{4}=(-1 / 2+u,-1 / 2+u,-1 / 2)$ mit $u \approx 0,31$ nach [Mat76].

$\mathrm{IrO}_{2}$ liegt als feines schwarzes Pulver vor, wenn es durch Kondensation des flüchtigen Trioxids $\mathrm{IrO}_{3}$ hergestellt wird. Es kann auch durch Glühung von Ir bei Temperaturen oberhalb von $550^{\circ} \mathrm{C}$ an der Oberfläche nachgewiesen werden. Da jedoch der Oxidationsprozess bei niedrigen Temperaturen zwischen $550^{\circ}$ und $800^{\circ} \mathrm{C}$ diffusionslimitiert ist und sich ein thermisch instabiles, nicht stöchiometrisches $\mathrm{IrO}_{2-x}$ an der Oberfläche bildet, dessen Struktur ebenfalls nicht eindeutig geklärt werden konnte, eignet sich diese Methode nicht zur $\mathrm{IrO}_{2}$ Schichtherstellung [Cha00], [Peu84]. Bei höheren Temperaturen $\mathrm{T}>800^{\circ} \mathrm{C}$ tritt bereits ein nachweisbarer Massenverlust des $\mathrm{zu} / \mathrm{IO}_{\mathrm{x}}$ oxidierten $\mathrm{Ir}$ durch Volatilisation von $\mathrm{IrO}_{3}$ ein [Kri63], [Cho71]. Ir schmilzt bei $2443^{\circ} \mathrm{C}$ [Röm97], für $\mathrm{IrO}_{2}$ wird von Cha et al. [Cha99] der kritische temperaturabhängige Sauerstoffpartialdruck für die Dissoziationsreaktion

$\operatorname{IrO}_{2}(s) \rightarrow \operatorname{Ir}(s)+O_{2}(g) \quad, K_{p}=p\left(O_{2}\right)$

mit

$$
\begin{aligned}
\log \left(p\left(O_{2}\right) / \mathrm{atm}\right)= & 4,401-1,397 \cdot 10^{-3} \cdot T / K-11450 \cdot(T / K)^{-1}-4372,5 \cdot(T / K)^{-2} \\
& +1,787 \cdot \log (T / K)
\end{aligned}
$$

angegeben. Dabei bezeichnet $\mathrm{K}_{\mathrm{p}}$ die Gleichgewichtskonstante der Reaktion [Kub67]. Aus dieser Abhängigkeit ergibt sich eine Dissoziationstemperatur bei AtmosphärenSauerstoffpartialdruck von etwas unterhalb $1100^{\circ} \mathrm{C}$, in guter Übereinstimmung mit [Gme78] und [Sam82]. Es kann sich aber schon bei Temperaturen über $800^{\circ} \mathrm{C}$ in einer Umgebung mit geringem Sauerstoffpartialdruck ein gewisses Sauerstoffdefizit $\mathrm{IrO}_{2-x}$ einstellen [Cor62]. 
Die Bildungsenthalpie der Übergangsmetalloxide wie die des $\mathrm{IrO}_{2}$ ist vergleichbar mit der der Übergangsmetallnitride [Kru83]. Sie beträgt nach der chemischen Gleichung bei Raumtemperatur [Ans98]

$\mathrm{Ir}+\mathrm{O}_{2} \rightarrow \mathrm{IrO}_{2} \quad, \Delta \mathrm{H}=-242,7 \mathrm{~kJ} / \mathrm{mol}$

Neben den hier erwähnten Eigenschaften wurde an kristallinen und amorphen $\mathrm{IrO}_{2}$ Schichten weiterhin Elektrochromismus nachgewiesen. Diese Eigenschaft macht das Material ebenfalls für die Anwendung in Displays und optischen Bauelementen interessant [Nis97], [Ben83]. Weitere mögliche Anwendungen sind aufgrund der gefundenen elektrochemischen Eigenschaften der Einsatz als pH-Sensoren [Kat82], [Kre86] oder als Elektroden für neurale Stimulation [Rob83], [Rob86], [Bee88].

\subsection{Dünne $\mathrm{IrO}_{2}$ Schichten}

Es existieren bislang nur wenige Veröffentlichungen zur reaktiven Sputterdeposition von $\mathrm{IrO}_{2}$, da das $\mathrm{Ir} / \mathrm{IrO}_{2}$ System erst seit Mitte der 90er Jahre als neues Materialsystem für die Integration in die Si-Technologie diskutiert und erprobt wird [Cho97], [Lia98]. Einige grundlegende Untersuchungen zur Abscheidung mittels reaktiver Sputterdeposition werden von Cho et al. [Cho97] angestellt, es bleiben jedoch noch viele grundlegende Aspekte, beispielsweise ein verallgemeinertes Verfahren zum Auffinden der optimalen Depositionsbedingungen, Analysen zu Schichtspannungen und deren Relaxation, sowie das Rekristallisationsverhalten offen. Das dem Ir//rO $\mathrm{ra}_{2}$ ähnliche $\mathrm{Ru} / \mathrm{RuO}_{2}$ System ist schon seit Ende der $80 \mathrm{er}$ Jahre, allerdings zunächst als Interdiffusionsbarriere für Al und Si untersucht worden [Kru88], [Kol87]. Aus diesem Grund ist es intensiver in Bezug auf reaktive Sputter-Abscheidung [Kag98], Schichtstruktur und -morphologie [Lee96], Leitfähigkeit [Kru89] und Schichtspannungen [Des99] charakterisiert worden.

Bislang sind an dünnen $\mathrm{IrO}_{2}$ und $\mathrm{RuO}_{2}$ Schichten keine quantitativen Ergebnisse zur Sauerstoffselbstdiffusion veröffentlicht worden, obwohl der Einsatz von elektrisch leitfähigen $\mathrm{IrO}_{2}$ Schichten als Sauerstoffdiffusionsbarriere bereits 1994 von Nakamura et al. [Nak94a] vorgeschlagen worden ist. Insofern ist eine Analyse der Sauerstoffselbstdiffusion in Verbindung mit einer schichtmorphologischen Charakterisierung durch Transmissionselektronenmikroskopie (XTEM), die über die bereits veröffentlichten Röntgendiffraktometrie- (XRD) und Rasterelektronenmikroskopie- (REM) Resultate hinausgeht, wünschenswert. Überdies ist in der Literatur bislang eine Mehrzahl von Vorschlägen und Konzepten zur Integration von oxidischen Dielektrika bzw. Ferroelektrika erschienen [Son00], [Yam97]. Bislang sind aber keine grundlegenden Experimente veröffentlicht, die Aufschluss über die Diffusionswege und die Ausfallursache von vollintegrierten Sauerstoffbarrieren geben. Es bleibt also zu klären, was 
der dominierende Ausfallgrund ist, und auf welche Weise eine erfolgreiche Integration auf der Basis einer 0,6 $\mu \mathrm{m}$-Technologie zu realisieren ist. 


\section{Charakterisierungsmethoden}

\subsection{Prozess-Überwachung}

Unter den prozessüberwachenden Charakterisierungsmethoden versteht man die Kontrolle der Schichteigenschaften, die ohne allzu großen Aufwand zu erfassen sind und unter Produktionsgesichtspunkten die Prozessstabilität gewährleisten. Dazu gehören die Messung der Schichtdicke, des Schichtwiderstandes und der Schichtspannung. Besonders die Analyse der Schichtspannung ist ein sehr sensitives Verfahren, um Veränderungen in der Abscheidung zu erkennen.

\subsubsection{Röntgenreflektometrie (XRR)}

Mittels Röntgenreflektometrie kann neben der Dichte und der Rauigkeit auch die Dicke einer Schicht bestimmt werden. Dazu lässt man Röntgenstrahlung unter streifendem Einfall auf das beschichtete Substrat treffen und nutzt die Interferenz der an Schichtoberseite und Schicht-Substrat-Grenzfläche reflektierten Röntgenstrahlung aus. Die Totalreflektion von Röntgenstrahlen ist aufgrund des Brechungsindex, der nach

$n=1-\delta-i \beta$

kleiner als 1 ist, nur bei sehr kleinem Winkel zu beobachten, da $\delta$ und $\beta$ positive Werte sind, die die Dispersion und die Absorption beschreiben. Die Auswertung eines Reflektometriespektrums kann einerseits per Auswertung der Abstände der bei konstruktiver Interferenz entstehenden Maxima im Spektrum nach der Formel

$\theta_{m}^{2}=\theta_{c}^{2}+\frac{\lambda^{2}}{4 d^{2}} \cdot m^{2} \quad, m=0,1,2, .$.

durchgeführt werden [Kie31]. Dabei bezeichnet $\theta_{m}$ den Glanzwinkel des m-ten Intensitätsmaximums, $\theta_{c}$ den Totalreflektionswinkel, $\lambda$ die Wellenlänge des benutzten monochromatisierten Röntgenlichts und d die Schichtdicke. Über die Lage des Totalreflektionswinkel $\theta_{c}$ kann die röntgenographische Dichte $\rho$ der Schicht bestimmt werden, die mit der Dispersion $\delta$ in folgendem Zusammenhang steht [Zor94]

$\theta_{c}=\sqrt{2 \cdot \delta}=\left(\frac{N_{A} \cdot r_{0}}{\pi} \cdot \frac{\rho\left(Z+f^{\prime}\right)}{A} \lambda^{2}\right)^{1 / 2}$

Dabei ist $N_{A}$ die Avogadro-Konstante, $r_{0}$ der klassische Elektronenradius, $Z$ die Ordnungszahl, $A$ die atomare Masse und $f^{\prime}$ die Dispersionskorrektur. 
Bei Vielfachschichten verwendet man jedoch zur Vereinfachung der Auswertung der Spektren eine Simulation. Die Spektren der in dieser Arbeit untersuchten Schichten wurden mit der auf den Veröffentlichungen von Kiessig [Kie31], Parratt [Par54] und Nevot [Nev80] basierenden Software Refsim V1.2k (Bruker-AXS GmbH) simuliert ${ }^{16}$, um parallel Informationen über die Rauigkeit und die Schichtdichte zu erhalten [Zor94].

Dieses Verfahren ist nur durch zwei Einschränkungen limitiert. Sehr dicke Schichten führen zu einem zu starken Intensitätsverlust und deswegen zu einem schlechten Signal/Rauschverhältnis, sehr raue Schichten zerstören die Interferenzerscheinung. Der relative Fehler bei der Schichtdickenbestimmung mittels XRR ist gewöhnlich geringer als 1\%, die erreichbare Genauigkeit für die Dichtebestimmung liegt etwa bei $0,03 \mathrm{~g} / \mathrm{cm}^{3}$ und der Fehler bei der Bestimmung der Rauigkeit ${ }^{17}$ liegt typischerweise bei $\pm 0,1 \mathrm{~nm}$ [Zor94]. Durchgeführt wurden die Analysen an einem Siemens D5000 Röntgengerät (heute Bruker-AXS).

\subsubsection{Profilometrie}

Die Methode basiert auf der Abtastung der Probe mit einer Nadel, die sich im mechanischen Kontakt mit der Schicht befindet. Dieses Höhensignal wird über ein Hebelsystem verstärkt und piezoelektrisch oder kapazitiv ausgelesen. Um die Profilometrie als Methode zur Schichtdickenbestimmung einzusetzen, ist eine Stufe in der zu untersuchenden Schicht notwendig. Diese Stufe wird durch Aufbringen eines Filzstiftstriches auf das Substrat hergestellt. An der so markierten Stelle kann die Schicht lokal leicht mit Ethanol entfernt werden, so dass sich daraus eine mittels Profilometer auszumessende Stufe ergibt. Vergleichsmessungen an deponierten Schichten zwischen Röntgenreflektometrie und Profilometrie zeigen, dass die Schichtdickenbestimmung mit den beiden Methoden sehr gut übereinstimmende Resultate mit einem relativen Fehler von etwa $2 \%$ liefern. Für die Profilometrie-Analyse wurde ein Alpha-Step 20 Profilometer der Firma TENCOR ${ }^{18}$ eingesetzt. Das Messverfahren wurde in dieser Arbeit auch standardmäßig zum Ausmessen von Sputterkratern bei SIMSTiefenprofilanalysen verwendet.

\subsubsection{Vierpunkts-Schichtwiderstandsmessungen}

Die Schichtwiderstandsdaten wurden mit einem Omnimap 20 Vierspitzenmessgerät der Firma Prometrix durchgeführt. Die Messung besteht aus 49 radialsymmetrisch über den Wafer verteilten Einzelmessungen, um gleichzeitig Aussagen über die Schichthomogenität auf dem beschichteten Substrat machen zu können. Bei der Messung wird an die zwei äußeren der

\footnotetext{
${ }^{16}$ B. Jobst, Siemens AG, Abt. CT MM 7

${ }^{17}$ gemeint ist die mittlere quadratische Rauigkeit (s. Kap. 4.6)

${ }^{18}$ KLA-Tencor Corporation, www.tencor.com
} 
vier in einer Reihe mit jeweils gleichem Abstand voneinander angeordneten Spitzen ein Strom von typischerweise einigen $\mathrm{mA}$ eingeprägt und die entstehende Potentialdifferenz zwischen den beiden inneren Spitzen gemessen. Als Schichtwiderstand erhält man durch Lösen der Poisson-Gleichung nach Smits [Smi58]

$$
R_{S}=\frac{\pi}{\ln 2} \cdot \frac{U}{I}
$$

Bei bekannter Schichtdicke d lässt sich über die Formel

$$
\rho=R_{S} \cdot d=\frac{\pi}{\ln 2} \cdot \frac{U}{I} \cdot d
$$

aus dem gemessenen Schichtwiderstand $R_{S}$ der spezifische Widerstand $\rho$ errechnen.

\subsubsection{Messungen der mechanischen Schichtspannungen}

Die Spannungsmessungen wurden an einem Ultra Gage 9500 Messgerät der Firma ADE durchgeführt. Mit diesem Messgerät wird die Verbiegung b der Probe $\sqrt{19}$ vor und nach einem Prozessschritt mit einem kapazitiven Verfahren gemessen [Zha95]. Dabei trägt man der Tatsache Rechnung, dass die benutzten Silizium (100)-Wafer im Ausgangszustand im allgemeinen nicht vollständig plan sind. Bei der Messung wird das Substrat zwischen zwei die Position des Wafers kapazitiv messenden Elektroden hindurchrotiert; durch Aufnahme von mehr als 10000 Einzelmesspunkten wird der Waferkrümmung eine Kugel mit einem Krümmungsradius zugeordnet. Der Zusammenhang zwischen Verbiegung b und differentiellem Krümmungsradius $\mathrm{R}$ kann aus der folgenden geometrischen Überlegung berechnet werden

$$
b=R-R \cos \phi=R \cdot\left(1-\cos \frac{D / 2}{R}\right) \cong R \cdot\left(1-1+\frac{D^{2}}{8 R^{2}}\right)=\frac{D^{2}}{8 R},
$$

wobei D der Durchmesser des Wafers ${ }^{20}$ ist und $\phi$ der halbe Öffnungswinkel des Wafers vom Mittelpunkt der einbeschriebenen Kugel. Unter der Annahme, dass die Schicht vollständig auf dem Substrat haftet, kann man mit Hilfe der Stoney-Gleichung [Sto09]

$$
\sigma=\frac{1}{6} \frac{E_{S i}}{1-v_{S i}} \frac{d_{s}^{2}}{d_{f}} \frac{1}{R}
$$

\footnotetext{
19 bow (engl.): Verbiegung, Krümmung

${ }^{20}$ In dieser Arbeit wurden nur Silizium Substrate mit einem Durchmesser von $\mathrm{d}=150 \mathrm{~mm}$ verwendet
} 
und den darin enthaltenen bekannten Größen $E_{S i}, v_{S i}, d_{f}$ und $d_{S}$, die den Elastizitätsmodul, die Poissonzahl des Si-Wafers, die Schicht- und die Substratdicke darstellen, die integrale Schichtspannung $\sigma$ errechnen. Dabei ist

$\frac{1}{R}=\frac{1}{R_{1}}-\frac{1}{R_{2}}=\frac{R_{2}-R_{1}}{R_{1} R_{2}}$

mit $R_{1}$ als Krümmungsradius der Referenzmessung und $R_{2}$ als Radius der Messung nach dem Prozessschritt.

\subsection{Stöchiometrie, Chemische Analyse, Tiefenprofilanalysemethoden}

Mit den hier genannten Analysemethoden wurden die chemischen Eigenschaften der Proben sowohl im Ausgangszustand, also direkt nach der Sputterdeposition, als auch nach thermischer Nachbehandlung charakterisiert. Mit den Tiefenprofilanalysemethoden AugerElektronen-Spektroskopie (AES) und Sekundär-lonen-Massen-Spektrometrie (SIMS) ist es möglich, die Diffusion des Sauerstoffs sowie Interdiffusionsreaktionen zu analysieren.

\subsubsection{Rutherford Rückstreu Spektrometrie (RBS)}

Die RBS-Analyse wurde in dieser Arbeit benutzt, um die chemische Zusammensetzung der gesputterten dünnen Schichten zu bestimmen. Dazu wird die nicht-resonante RutherfordStreuung [Chu78] von monoenergetischen $\mathrm{He}^{2+}$ Ionen an den $\mathrm{zu}$ untersuchenden $\mathrm{IrO}_{\mathrm{x}}$ Schichten energiesensitiv gemessen. Das Rückstreuspektrum wird mit einer Software simuliert, in die die Schichtparameter wie die Komposition und die Dicke, bzw. die Flächenbelegungsdichte der Schicht als Simulationsparameter eingehen.

Der für die RBS-Messungen 21 verwendete lonen-Beschleuniger ist ein Tandetron, Modell 4110 der Firma General Ionex Corporation 22 mit einer maximalen Beschleunigungsspannung von $\mathrm{U}=1,1 \mathrm{MV}$. Der Beschleuniger ist so aufgebaut, dass die mit einer Duoplasmatronquelle erzeugten He-Ionen auf eine maximale Energie von $\mathrm{E}=3,3 \mathrm{MeV}$ beschleunigt werden können [Dar92]. Für die Messungen an den $\mathrm{IrO}_{\mathrm{x}}$ Schichten wurden jedoch nur Spektren bei einer Primärionenenergie von $\mathrm{E}=2,2 \mathrm{MeV}$ aufgenommen, um resonante Streuung zu vermeiden, die bei voller Ausnutzung der Beschleunigungsspannung beispielsweise bei einer Energie von 3,05 MeV auftreten würden [Chu78]. In der gewählten Messgeometrie werden die rückgestreuten lonen unter einem Streuwinkel von $\vartheta=170^{\circ}$ detektiert, für die Untersuchung der Proben wurde eine zeitaufintegrierte Ladung von $200 \mu \mathrm{C}$ verwendet. Bei der Messung wurde

\footnotetext{
${ }^{21}$ Dr. W. Hösler, Dr. F. Bleyl, Siemens AG, Abt. CT MM 7

${ }^{22}$ später von der Firma High Voltage Engineering Europa übernommen
} 
mittels computergesteuerter Goniometer-Orientierung des Si(100) Substrats das vom Silizium herrührende Messsignal minimiert („Channeling“), um die Genauigkeit der Sauerstoffbestimmung bei der Simulation zu erhöhen. Die Simulation der Spektren geschah mittels der Software RUMP [Doo85]. Die verwendeten Substrate waren Si(100) Wafer mit natürlichem Oxid, diese wurden unmittelbar vor der Schichtpräparation mit 0,1 molarer Flusssäure (HF) angeätzt, um das natürliche Oxid zu entfernen.

\subsubsection{Auger-Elektronen-Spektroskopie (AES)}

Die Auger-Elektronen-Spektroskopie kann elementspezifisch die Oberfläche eines Festkörpers bzw. einer dünnen Schicht analysieren. Man bedient sich dabei des Prinzips, dass Primärelektronen mit einer ausreichenden Energie die Atome in der zu untersuchenden Oberfläche ionisieren können. Das Atom ist dann in einem angeregten Zustand und kann aus diesem angeregten Zustand unter Aussendung von für das angeregte Atom charakteristischen Photonen oder durch den Auger-Prozess wieder in den Grundzustand zurückkehren. Bei der Auger-Analyse detektiert man energiespezifisch die Anzahl der bei dem gleichnamigen Prozess ausgesandten Elektronen. Aus dieser Information kann man auf die Bindungsenergie, die ebenfalls charakteristisch für das jeweilige Element ist, und damit auf das angeregte Element schließen. Die Intensität der Auger-Signale ist ein Maß für die Anzahl der angeregten Atome, d.h. man kann über eine geeignete Kalibrierung der Signalhöhen mit einer Referenzprobe eine quantitative Element-Bestimmung vornehmen.

Die Auger-Elektronen-Signale befinden sich in einem direkt aufgenommenen Spektrum auf dem Untergrund der rückgestreuten Elektronen. Um auch die intensitätsschwachen Signale deutlich vom Untergrund zu trennen und um dicht beieinander liegende Signale voneinander zu unterscheiden, wird generell das differentielle Spektrum ausgewertet. Hierbei ist die Ordinate nicht mit $\mathrm{N}(\mathrm{E})$, also der Zählrate skaliert, sondern mit deren Ableitung nach der Energie. Nach Konvention wird das Minimum der Ableitung eines Messsignals im differentiellen Spektrum für die Definition der energetischen Lage des Signals benutzt [Bis89]. Als Maß für die Intensität eines Signals wird die Maximum-Minimum-Differenz (Signalhub) verwendet.

Die Ergebnistiefe der Auger-Elektronen beschränkt sich auf wenige nm, da für die Analyse nur Elektronen ohne Energieverlust zur Elementanalyse benutzt werden. Außerdem kann es vorkommen, dass sich gewisse Auger-Signale der zu messenden Elemente (z.B. Ti und N) überlappen. Dazu ist es mittels einer Software notwendig, die gemessenen Signale zu trennen. Durch die Fähigkeit, dass die Auger-Analyse verschiedene Bindungszustände eines Elementes auflösen kann, ist es zusätzlich möglich, eventuell neu entstandene Phasen nachzuweisen. Ein Signal von chemisch anders gebundenen Atomen eines Elements unterscheidet sich im allgemeinen durch eine beobachtbare Energieverschiebung und eine Veränderung der Signalform. 
Die für die Probencharakterisierung benutzte Auger-Anlage ist eine kommerzielle UHVAnlage PHI 660 von Perkin Elmer. Die Messkammer wird über eine Kombination aus Vorpumpe, Turbomolekularpumpe und Ionengetterpumpe auf Ultrahochvakuumbedingungen $p \approx 10^{-9}$ Torr evakuiert, um Restgaseinflüsse und Wiederbedeckung der Oberfläche während der Tiefenprofilanalysen zu minimieren. Für die Analyse ${ }^{23}$ wird mit einem Primärstrom von $I_{P}=10 \mu \mathrm{A}$ und einer Beschleunigungsspannung von $U_{B}=10 \mathrm{kV}$ gearbeitet. Die AugerElektronen werden in einem Energiefenster von $E=20 . .2270 \mathrm{eV}$ detektiert. Bei Tiefenprofilanalysen wird die Probe um $\alpha=30^{\circ}$ gegen die Primärelektronenquelle verkippt und schließt mit der lonenquelle einen Winkel von etwa $\beta=15^{\circ}$ ein. Während des Sputterabtrags durch die Ar-lonen wird die Probe dabei durch einen Motor um die Schichtebenennormale rotiert, um eine Aufrauung der Oberfläche durch einseitigen seitlichen lonenbeschuss zu vermeiden und dadurch eine deutlich verbesserte Tiefenauflösung zu erzielen. Für die Tiefenprofilierung benutzt man eine Methode, die abwechselnd eine vor der Messung festgelegte Zeit sputtert und während der Abtragspause die Auger-Messung vornimmt. Dabei misst man die Energiefenster der Elemente, deren Tiefenprofil aufgenommen werden soll. Zunächst werden die reaktivsten Elemente gemessen (hier vor allem Ti), um chemische Reaktionen durch Wiederbedeckung von flüchtigen Komponenten (z.B. $\mathrm{O}_{2}$ ) zu minimieren. Die gemessenen Spektren wurden mit einer Multipak Software von Physical Electronics 24 ausgewertet.

An der Anlage befindet sich seitlich ein zweiter Sekundär-Elektronen-Detektor, mit dem REM-Aufnahmen der Probenoberfläche gemacht werden können. Die von der Oberfläche emittierten Sekundärelektronen in der verwendeten UHV-Anlage werden jedoch nicht wie in einem gewöhnlichen REM mit einer zusätzlichen Spannung beschleunigt, was eine reduzierte Bildqualität bedingt.

\subsubsection{Sekundär-Ionen-Massen-Spektrometrie (SIMS)}

Die Sekundär-Ionen-Massen-Spektrometrie (SIMS) ist eine sehr nachweisempfindliche Methode, um Tiefenprofile von Elementen aufzunehmen. Die Nachweisgrenze liegt bei etwa $0,01 \mathrm{ppm}$ bis $10 \mathrm{ppm}$ [Cri85]. Durch die Messmethode ist sowohl eine sehr hohe Tiefenauflösung als auch eine sehr gute laterale Ortsauflösung von $<1 \mu \mathrm{m}$ möglich. Dabei weist man die durch Primärionenbeschuss aus der Probe austretenden Sekundärionen nach. Diese können durch massenspektrometrische Trennung nach M/e-Verhältnis separiert über Detektoren nachgewiesen werden. Diese Methode wurde in dieser Arbeit dafür benutzt, um Sauerstoff-Tracer-Diffusionsprofile in den gesputterten $\mathrm{IrO}_{2}$ Schichten aufzunehmen.

\footnotetext{
${ }^{23}$ Dr. W. Hösler, Dr. F. Bleyl, Siemens AG, Abt. CT MM 7

${ }^{24}$ Physical Electronics, Inc., 6509 Flying Cloud Drive, Eden Prairie, MN 55344
} 
Die präsentierten SIMS-Profile sind mit einer Cameca IMS 6f Anlage aufgenommen worden 25 . Für die Messungen wurde eine Beschleunigungsspannung für die $\mathrm{Cs}^{+}$-Primärionen von $U_{P}=10 \mathrm{kV}$ benutzt. Dabei wird aber die zu untersuchende Probe zusätzlich auf eine negatives Potential von $U=-5 \mathrm{kV}$ gelegt, so dass die Primärionenenergie tatsächlich $E_{P}=15 \mathrm{keV}$ beträgt. Der Primärionenstrom, der die Abtragsgeschwindigkeit bestimmt, wurde auf einen Wert von $I_{P}=45 \mathrm{nA}$ eingestellt. Dieser Wert stellt einen Kompromiss zwischen einer adäquaten Datenpunktdichte und dem zu minimierenden Sauerstoffuntergrund dar, der bei zu langsamer Abtragsrate die Messqualität reduziert. Die quadratische Rastergröße, die die effektiv abgetragene Fläche der Schicht darstellt, betrug $150 \mu \mathrm{m}$ und die eigentliche Sonde, die durch die lonenoptik bestimmt wird, hatte einen Durchmesser von $d=60 \mu \mathrm{m}$. Das von Cameca verwendete doppeltfokussierende Massenspektrometer, welches ausführlich z.B. von Sykes [Syk89] beschrieben ist, misst inkrementell nach Massenzahl die Elemente durch und beginnt im nächsten Messschritt wieder bei der kleinsten Masse. Diese MassenDurchstimmung kann jedoch nicht beliebig schnell geschehen und bringt bei hohen Massendifferenzen $(\mathrm{m}(\mathrm{Ir})=193 \mathrm{u}$ ) eine Vergrößerung des Zeitintervalls $\Delta t$ zwischen zwei SauerstoffMesspunkten mit sich. Um eine möglichst hohe Datenpunktdichte für die Sauerstoffprofile zu erreichen, wurde daher bei den $\mathrm{IrO}_{2}$ Schichten kein Ir-Signal mitgemessen. Als Detektoren für die Sekundärionen benutzt man im Messbereich bis $10^{6}$ Zählereignissen pro Sekunde (cps) einen Elektronenmultiplier. Für höhere Zählraten ist die Zählcharakteristik dieser Detektoren nicht mehr linear, man setzt dafür einen Faraday-Cup ein.

Für die Umskalierung der Ordinate (Sputterzeit) in die tatsächliche analysierte Tiefe wird nach der Analyse mittels eines Profilometers die Kraterendtiefe ausgemessen. Bei einer homogenen Schicht und konstanter Abtragsgeschwindigkeit kann man die Achse linear in eine Länge umskalieren. Da aber bei allen Analysen durch mehrere Schichten hindurchgemessen wurde und deswegen die Umskalierung aufgrund von unterschiedlichen Sputterraten verfälscht wird, wurden zur Tiefeneichung die nominellen Werte der einzelnen Schichtdicken herangezogen. Für die Messungen von Isotopensauerstoff ${ }^{18} \mathrm{O}$ in $\mid r^{16} \mathrm{O}_{2}$, ist wegen der Überlagerung des ${ }^{18} \mathrm{O}$ Signals mit dem $\mathrm{H}_{2}{ }^{16} \mathrm{O}$ Untergrundsignal des UHV Restgases eine hohe Massenauflösung von $M / \Delta M \approx 1900$ nötig.

\subsection{Mikrostruktur und Schichtmorphologie}

Die Analysen mittels Röntgendiffraktometrie (XRD) und Transmissionselektronenmikroskopie (TEM) sind etablierte Methoden, um eine Phasencharakterisierung von dünnen Schichten vorzunehmen und Informationen über das Gefüge zu erhalten. Ergänzend zu diesen Analy-

${ }^{25}$ Dr. F. Jahnel, B. Weidinger, Siemens AG, Abt. CT MM 7 
semethoden wurden rasterkraftmikroskopische Messungen an den Schichten vorgenommen, um die Schichtmorphologie zu untersuchen und die Ergebnisse untereinander zu vergleichen.

\subsubsection{Röntgendiffraktometrie (XRD)}

Mit der Röntgendiffraktometrie ist es möglich, dünne Schichten zerstörungsfrei und ohne Präparationsaufwand mikrostrukturell zu charakterisieren und Informationen über Phasen, Gitterkonstanten und Korngrößen zu erhalten. Zusätzlich können aus den gemessenen Diffraktometriespektren Aussagen zur Schichttextur und auch zu Schichtspannungen gemacht werden. Dazu werden die beschichteten Substrate im allgemeinen in Bragg-BrentanoGeometrie oder unter streifendem Einfall („grazing incident“, GIXRD) analysiert. Es ist mittels XRD möglich, in den Proben vorhandene Phasen mit einem Volumenbruchteil bis zu wenigen Prozent nachzuweisen.

Zur Analyse der Spektren nutzt man die Bragg-Gleichung

$\lambda=2 d_{h k l} \sin \theta$

aus, mit der man den Beugungswinkeln atomare Netzebenenabstände zuweisen kann [Cul78]. In dieser Gleichung ist $d_{h k l}$ der Netzebenenabstand mit der Millerschen Indizierung (hkl) und $\theta$ stellt den Glanzwinkel dar, also den Winkel, den der Strahl mit der Substratebene einschließt. Durch die Anwendung der Scherrerformel [Cul78]

$d=\frac{0,9 \cdot \lambda}{B \cdot \cos \theta}$

ist es möglich, aus der beobachteten Reflexbreite B eine Kohärenzlänge $d$ zu errechnen und diese als Abschätzung für die mittlere Korngröße heranzuziehen. Diese Methode ist bei sehr großen Kristalliten durch die endliche apparative Winkelauflösung begrenzt.

Die in dieser Arbeit gezeigten Messungen wurden mit Siemens Diffraktometern D500 und D8 Advance $^{26}$ mit über Göbel-Spiegel monochromatisierter $C u-K_{\alpha}$ Strahlung in ParallelstrahlGeometrie durchgeführt27. Der symmetrische Strahlengang wurde anstelle der GIXRDMethode gewählt, um die Schichttexturen quantitativ auswerten zu können. Die Spektren wurden in $1 / 50^{\circ}$ Schritten zwischen $2 \theta=10^{\circ} . .65^{\circ}$ aufgenommen.

Für die in-situ Hochtemperaturmessungen (HTXRD) werden die Proben in einem von Siemens hergestellten D500 $\theta$ / $\theta$ Diffraktometer gemessen. In diesem Messgerät dreht sich der

\footnotetext{
${ }^{26}$ www.bruker-axs.com

${ }^{27}$ B. Jobst, CT MM 7
} 
heizbare Probenträger nicht, bei der Messung wird vielmehr die Röntgenröhre und der Detektor um den gleichen Winkel $\theta$ gegen die Probe gekippt, so dass die Bragg-Gleichung angewendet werden kann. Die Proben werden mit einer Rate von $r=12 \mathrm{~K} / \mathrm{min}$ in normaler Umgebungsatmosphäre im niedrigen Temperaturbereich in $100^{\circ} \mathrm{C}$ - bzw. im Hochtemperaturbereich in $50^{\circ} \mathrm{C}$-Schritten bis auf eine Maximaltemperatur von $850^{\circ} \mathrm{C}$ geheizt. Während der Röntgenmessung, für die ein Bereich von $20^{\circ}<2 \theta<60^{\circ}$ gewählt wurde, wird die angefahrene Temperatur konstant gehalten. Als Schrittweite wurde $\Delta(2 \theta)=0,05^{\circ}$ gewählt, um die Messzeit kurz zu halten.

\subsubsection{Transmissionselektronenmikroskopie ((X)TEM)}

Die Transmissionselektronenmikroskopie erlaubt durch elektronenoptische Abbildung eine direkte ortsaufgelöste Analyse der Mikrostruktur der Proben. Im Vergleich zur Röntgendiffraktometrie, die integral über das beleuchtete Probenvolumen misst, kann man lokal, auch hochaufgelöst, Informationen über einzelne Körner, Defekte und Grenzflächen erhalten. Mittels quantitativer Auswertung von Elektronenbeugungsbildern ist es möglich, dieselben Informationen wie aus einem Weitwinkelröntgenbeugungsspektrum zu erhalten, allerdings mit deutlich feinerer Sonde. Ein Nachteil der Analyse mit dem TEM ist der präparative Aufwand, um ohne signifikante Schädigung der Schicht ausreichend dünne Probenstücke für die Durchstrahlung herzustellen. Eine gute Darstellung der sehr umfassenden Thematik der Transmissionselektronenmikroskopie bietet Alexander [Ale97].

Für die TEM Analysen 28 wurde ein hochauflösendes Elektronenmikroskop mit Feldemissionskathode des Typs CM200-FEG-UT von Philips ${ }^{29}$ benutzt, das mit einer Beschleunigungsspannung von $U=200 \mathrm{kV}$ arbeitet. Das Gerät hat eine Punkt-Auflösung von 0,188 nm und eine untere Informationsgrenze von 0,11 nm [Sei98]. Es wurden Probenstücke sowohl für die Analyse im plan-view Modus, also mit Durchstrahlung in Schichtnormalenrichtung, als auch im Querschliff präpariert und analysiert.

Die im Querschliff analysierten Proben wurden mit einer Diamantdrahtsäge in etwa $300 \mu \mathrm{m}$ dicke Scheiben zersägt. Anschließend wurden diese und die für die elektronenmikroskopische Aufsicht-Analyse bestimmten Proben durch Schleifen und Polieren mit Hilfe einer Diamantpaste mechanisch auf eine Dicke von ca. $30 \mu \mathrm{m}$ gedünnt. Darauf wurden die Proben durch Ionen-Ätzen mit Argonionen einer Energie von $E=4 \mathrm{keV}$ weitergedünnt, bis ein mit Elektronen durchstrahlbaren Bereich der Schicht erzeugt ist. Dabei wurde für die Aufsicht-

\footnotetext{
${ }^{28}$ Die TEM Analysen wurden in Zusammenarbeit mit Herrn Dr. M. Seibt, IV. Physikalisches Institut der Universität Göttingen, durchgeführt.

${ }^{29}$ Philips $\mathrm{GmbH}$, Deutschland, www.philips.de
} 
analysen eine Dual Ion Mill (Model 600) Ätzanlage der Firma Gatan ${ }^{30}$ benutzt, für die Querschliffpräparation wurde eine RES 010 der Firma Bal-Tec ${ }^{31}$ gewählt. Der Einschusswinkel wurde bei der Ätzpräparation unter $17^{\circ}$ gehalten, um eine signifikante Veränderung der Probe durch den Ätzprozess zu vermeiden.

\subsubsection{Rastertunnelmikroskopie (STM)}

Bei der Tunnelmikroskopie wird der Effekt des Tunnelns von Elektronen zwischen zwei sich nicht im Kontakt befindlichen leitfähigen Materialien ausgenutzt. Der Effekt, dass die Elektronen durch eine Potentialbarriere mit einer gewissen Wahrscheinlichkeit tunneln können, wird durch die Quantenmechanik erklärt. Der Tunnelstrom nimmt exponentiell mit zunehmendem Abstand der Elektroden ab.

Die rastertunnelmikroskopischen Analysen wurden mit einem Nanoscope II von Digital Instruments 32 an Luft durchgeführt. Ein rohrförmiger piezoelektrischer Kristall regelt dabei sowohl die vertikale als auch die laterale Bewegung, nachdem die Grobannäherung der Spitze an die Probe durch einen Schrittmotor erfolgt ist. Die Spitze wird aus einem 0,25 mm dicken Draht, der aus einer Pt/lr-Legierung ${ }^{33}$ besteht, mechanisch gezogen. In dem verwendeten "constant current mode" wird die Sonde an die Probe bis auf wenige nm angenähert und der Abstand Probe - Tunnelspitze mittels PI-Regelung durch einen fest gewählten Tunnelstrom eingestellt. Die Reglerspannung, die nötig ist, einen konstanten Tunnelstrom aufrechtzuerhalten, setzt die Software des benutzten PCs in eine Farbskala um. Mit der Rastertunnelmikroskopie werden nach Tersoff und Hamann [Ter83], [Ter85] die Elektronenzustandsdichte an der Fermikante spektroskopiert. Für die Untersuchung der Oberflächentopographie wird das PI-Reglersignal, das die elektronischen Eigenschaften der Festkörperoberfläche widerspiegelt, als Probentopographie interpretiert. Die über einen PC aufgenommenen Bilder bestehen aus 400x400 Punkten, von denen ein konstanter x- und y-Gradient zur Untergrundkorrektur abgezogen wird („planefit“). Eine detaillierte Beschreibung des verwendeten Tunnelmikroskops gibt Geyer [Gey92].

\subsubsection{Rasterelektronenmikroskopie (REM)}

Die Rasterelektronenmikroskopie wurde als zusätzliches Verfahren zur optischen Mikroskopie eingesetzt, um Oberflächentopologie- und Defektanalysen der gesputterten und thermisch nachbehandelten Schichten durchzuführen. Das Verfahren basiert auf der für die

\footnotetext{
${ }^{30}$ Gatan Inc., USA, www.gatan.com

${ }^{31}$ Bal-Tec AG, Liechtenstein, www.bal-tec.com

${ }^{32}$ Digital Instruments Inc., Santa Barbara, California, USA

${ }^{33}$ Im Volumen-Verhältnis $\mathrm{Pt} / \mathrm{Ir}=80 / 20$, Firma ChemPur $\mathrm{GmbH}$, Karlsruhe, Deutschland
} 
Oberflächentopologie charakteristischen Sekundärelektronenauslösung aus der Probe und zeichnet sich dadurch aus, dass man mit geringem Präparationsaufwand eine um ein Vielfaches höhere Vergrößerung zur konventionellen Lichtmikroskopie erreichen kann. Das für die Analysen verwendete Mikroskop ist ein SEM S-7800 von Hitachi und wurde mit einer Beschleunigungsspannung von $U_{B}=0,8 \mathrm{kV}$ und einem Primärelektronenstrom von $I_{P}=10 \mu \mathrm{A}$ betrieben.

\subsection{Mechanische und thermische in-situ Analysen}

\subsubsection{In-situ-Messungen der mechanischen Schichtspannungen}

Die in-situ-Spannungsmessungen wurden an einem TENCOR FLX-2900-Ofen $\frac{34}{\text { durchge- }}$ führt, der das beschichtete Substrat mit einer voreingestellten Heizrate auf eine gewünschte Endtemperatur heizt. Das Substrat liegt aus optischen Gründen mit der Schicht nach unten auf drei Quarzhaltern frei auf. Für die hier gemachten Analysen wurde auf eine mögliche Spülung des Ofens mit reinem $\mathrm{N}_{2}$ oder $\mathrm{O}_{2}$ verzichtet und in Umgebungsatmosphäre, also im wesentlichen einem $\mathrm{N}_{2} / \mathrm{O}_{2}$-Gemisch, gearbeitet. Während des Heizzyklus wird in bestimmten Zeitintervallen die beschichtete Seite des Wafers mit einer Laserdiode linear durch den Mittelpunkt des Wafers abgerastert. Durch die positionsempfindliche Detektion des reflektierten Strahls an 50 äquidistanten Stellen wird die Krümmung des Substrates berechnet. Über die Stoney-Gleichung (2.7) kann man daraus dann bei bekannter Schichtdicke die integrale Schichtspannung errechnen.

\subsubsection{Differentielle Thermische Analyse (DTA) mit simultaner Restgasanalyse (RGA) und thermogravimetrischer Analyse (TGA)}

Die thermischen Analysen wurden in Kombination mit Thermogravimetrie- und Restgasmassenspektroskopieanalysen an einem STA ${ }^{35} 449$ C Jupiter der Firma Netzsch ${ }^{36}$ durchgeführt. In dem Ofen des Gerätes befindet sich ein $\mathrm{Al}_{2} \mathrm{O}_{3}$ Tiegel, an dem ein Pt/Rh Thermoelement angebracht ist. Zur Messung wird der Tiegel mit der zu messenden Probe beladen und während des Heizprozesses das differentielle thermische Signal des Tiegels aufgezeichnet und ausgewertet. Die Skalierung der Enthalpieachse wird über die Schmelzenthalpie mehrerer bekannter Eichsubstanzen kalibriert, die Temperaturachse über deren Schmelzpunkt.

Der Heizprozess bei den in dieser Arbeit gezeigten thermischen Analysen wird mit einer konstanten Temperaturrate von $r=10 \mathrm{~K} / \mathrm{min}$ durchgeführt. Dabei wird die Probe in einem Ar-

\footnotetext{
${ }^{34}$ KLA-Tencor Corp., USA, www.tencor.com

${ }^{35}$ Simultaneous Thermal Analyzer

${ }^{36}$ Netzsch Gerätebau GmbH, Selb, Deutschland; www.netzsch.com
} 
gongasfluss von $Q(A r)=10$ sccm $^{37}$ umspült. Während des Heizzyklus wird außerdem die Probe gewogen und der Restgasanteil des Sauerstoffs im Argonspülgas mittels eines Massenspektrometers ThermoStar der Firma Pfeiffer Vacuum ${ }^{38}$ gemessen. Die Wägung findet statt, um einen durch Verflüchtigung von Probenbestandteilen verursachten Gewichtsverlust nachzuweisen.

${ }^{37} \mathrm{sccm}$ : standard cube centimeters per minute (Standardkubikzentimeter pro Minute, s. Kap. 3.6)

${ }^{38}$ Pfeiffer Vacuum AG, Deutschland, www.pfeiffer-vacuum.de 


\section{Schichtdeposition}

\subsection{Depositionsverfahren}

Die wichtigsten Verfahren zur Dünnschichtabscheidung in der Siliziumtechnologie sind neben den chemischen Abscheideverfahren der Gasphasenabscheidung (CVD: Chemical Vapor Deposition) und dem Schleuderverfahren (Spin-Coating) die physikalischen Schichtdepositionsmethoden der Laserablation (PLD: Pulsed Laser Deposition) und des Sputterns (PVD: Physical Vapor Deposition). Das Sputterverfahren wird auch als Kathodenzerstäubung bezeichnet und bietet eine hohe Prozessflexibilität durch die Variation geeigneter Parameter zur gezielten Beeinflussung der Schichtcharakteristika. Außerdem besitzt das Verfahren eine sehr hohe Reproduzierbarkeit der physikalischen Schichteigenschaften wie Zusammensetzung, Leitfähigkeit und Schichtspannungen, was für die industrielle Produktion eine der wichtigsten Grundvoraussetzungen darstellt. Zudem sind für aufgesputterte (Metall-) Schichten das Schichtgefüge, die Morphologie und Schichtspannungen betreffende Modelle vorhanden [Tho74], [Tho75], [Win91]. Weiterhin gibt es für das reaktive Sputtern theoretische Konzepte, die konkrete Voraussagen über Plasmaeigenschaften und den Depositionsmodus ${ }^{29}$ und damit über die Schichtzusammensetzung zulassen [Zhu98], [Ros89], [Kus90], [Oka86], [Ber87]. Diese Argumente stellen die Hauptgründe dar, warum es sich gegenüber dem thermischen Aufdampfverfahren weitgehend durchgesetzt hat [Wid88]. Das in dieser Arbeit zur Abscheidung der Iridium- und Iridiumdioxidschichten benutzte Verfahren wird im folgenden vorgestellt.

\subsection{Sputterverfahren}

Der Effekt der Kathodenzerstäubung während einer Gasentladung wurde schon von Grove 1852 beobachtet [Gro52], [Gro53] und wird heute vielfältig, nicht nur in der Halbleitertechnologie, zur Beschichtung, Vergütung und Veredelung von Oberflächen benutzt. Als Sputtern bezeichnet man das Abstäuben von Target-Atomen aus einem Atomverband durch einen Impulstransfer energiereicher lonen. Dabei ist der Begriff Abstäuben als indirekte Emission in den Gasraum zu verstehen; indirekt deswegen, da nur ein kleiner Teil der Primärteilchen über einen direkten Stoß sekundäre Teilchen (Atome/Cluster) auslöst, sondern der eigentliche Sputterprozess vielmehr über Stoßkaskaden an der Oberfläche des Sputter-Targets abläuft. Die ausgelösten Atome haben typische kinetische Energien von einigen eV [Beh91].

\footnotetext{
${ }^{39}$ Mit Depositionsmodus ist hier in Abhängigkeit des reaktiven Gaspartialdrucks der Modus, in dem sich das Sputtertarget befindet, gemeint.
} 
Als Primärteilchen werden im allgemeinen Edelgasionen wie beispielsweise Ar oder $\mathrm{Ne}$ benutzt, um eine chemische Interaktion zwischen diesen mit dem abzustäubenden Material sowie der aufwachsenden Schicht zu verhindern. Dabei wird das Sputter-Target durch einen Gleich- oder Wechselspannungsgenerator auf ein negatives Potential gelegt, auf das die Edelgasionen beschleunigt werden und durch den Impulstransfer Target-Atome herauslösen. Das Anlegen eines Wechselfeldes an das Sputter-Target ermöglicht das Abscheiden von isolierenden Materialien: Beim sogenannten RF-Sputtern 40 wird im Gegensatz zum DCSputtern ${ }^{41}$ die asymmetrische Beweglichkeit der Elektronen und lonen im Plasma ausgenutzt, um eine kapazitive Aufladung an der Kathode zu generieren [Smi95].

Durch eine Magnetfeldunterstützung werden beim sogenannten Magnetronsputtern die Elektronen am Target durch die Lorentzkraft auf Spiralbahnen um die Magnetfeldlinien gezwungen, die im wesentlichen parallel zur Target-Oberfläche verlaufenden. Die erhöhte effektive Weglänge der Elektronen steigert die Stoßanzahl der Elektronen für die Erzeugung neuer Elektron/lonenpaare und führt somit zu einer Erhöhung der lonendichte und der Sputterausbeute. Außerdem kann dadurch der für die Schichtherstellung mögliche Arbeitsdruck und damit die Beschichtungszeit und eventuelle Verunreinigungen in der Schicht verringert werden. Durch die Verwendung von hochreinen Targets und Prozessgasen ist es somit möglich, reproduzierbar hochwertige und sehr reine Schichten herzustellen. Durch das sogenannte Biassputtern, also das Anlegen einer negativen Spannung an das Substrat, kann zusätzlich ein Rücksputtern zur Optimierung der Schichtmorphologie und Fülleigenschaften von strukturierten Substraten eingesetzt werden [Ros98].

\subsection{Reaktives Sputtern}

Um mehrkomponentige Oxid-, Nitrid- oder Halogenidschichten zu sputtern, gibt es einerseits die Möglichkeit, ein geeignetes Sputter-Target mit der gewünschten Stöchiometrie für die abzuscheidenden Schichten einzusetzen, andererseits ist es auch möglich, ein im einfachsten Fall einkomponentiges Material zu sputtern und dieses in einer reaktiven, z.B. sauerstoffhaltigen Atmosphäre während der Deposition reagieren zu lassen [Smi95]. Die erstgenannte Methode hat den inhärenten Nachteil, dass das reine einkomponentige Material mit dem Oxid-Target nicht abgeschieden werden kann, und weiterhin eine Variation in der Schichtzusammensetzung nur sehr begrenzt möglich ist. Außerdem wird experimentell vielfach nachgewiesen, dass die Target-Stöchiometrie durch physikalische Effekte während der Sputterdeposition nicht notwendigerweise 1:1 auf die Schicht übertragen wird [Sig81]. Die

\footnotetext{
${ }^{40}$ RF: radio frequency (Hochfrequenz)

${ }^{41}$ DC: directed current (Gleichstrom)
} 
zweitgenannte Vorgehensweise wird als reaktives Sputtern bezeichnet und wird auch für die Herstellung ternärer, quaternärer oder fünfkomponentiger Schichten genutzt [Tri96], [Tse01]. Durch das reaktive Sputtern erhält man die Möglichkeit, die Schichtzusammensetzung durch die Menge des in die Depositionskammer eingeführten reaktiven Gases graduell zu variieren und damit die physikalischen und chemischen Eigenschaften der Schicht gezielt zu beeinflussen. Die Flüsse der verwendeten Gase werden über geeichte Gasflussregler (MFC $)^{42}$ in die Depositionskammer eingeleitet, der sich in der Kammer einstellbare Partialdruckbereich kann über die Saugleistung der verwendeten Pumpe bestimmt werden.

Die Verwendung reaktiver Prozessgase kann unter bestimmten Umständen die eigentlichen Argon-Plasmaeigenschaften bzw. den Sputterprozess signifikant modifizieren. Durch die Existenz positiver Sauerstoffionen im Plasma beispielsweise kann einerseits ein zusätzlicher Sputterabtrag am Target verursacht werden. Andererseits kann bei nicht zu hohem Prozessdruck in planarer Sputtergeometrie ein Substratbombardement energiereicher Sauerstoffanionen eintreten, da aufgrund der hohen Elektronenaffinität des Sauerstoffs ${ }^{43}$ zusätzlich negative lonen wie $\mathrm{O}^{-}$oder $\mathrm{O}_{2}^{-}$im Plasma vorhanden sind [Wes89]. Die Sauerstoffanionen nehmen beim Durchlaufen des Kathodendunkelraums dabei typische Energien von mehreren 100 eV auf. Zusätzlich können energiereiche, am Sputter-Target reflektierte und neutralisierte Sauerstoffionen einen Substratbeschuss verursachen. Dieser Beschuss kann der Grund für Inhomogenitäten oder eine Nichtstöchiometrie der Schichten sein und auch die Schichtoberflächenmorphologie verändern [Pop95]. Weiterhin wird dieser Effekt als Möglichkeit für die Entstehung von Schichttexturen diskutiert, da das energetische lonenBombardement des Substrats zu einer Erhöhung der Oberflächenmobilität der Schichtatome führen kann [Pan98]. Zudem zeigen verschiedene Kristallflächen von polykristallinen Schichten aufgrund der unterschiedlichen Bindungsenergien im allgemeinen eine Anisotropie des Rücksputteryields 44 . Yu et al. [Yu86] haben gezeigt, dass bei der Sputterdeposition mit gleichzeitigem lonenbeschuss des Substrats die Schichttextur gesteuert werden kann. Dabei wachsen die Ebenen, die ein lonen-Channeling ${ }^{45}$ mit geringem Energieverlust in der loneneinschuss-Richtung zulassen, bevorzugt auf.

\footnotetext{
${ }^{42}$ Engl. Gasflussregler: mass flow controller

${ }^{43} E_{A}(O)=1,46 e V$ [Har78]

${ }^{44}$ Als Sputteryield $Y$ wird das Verhältnis von aus der Oberfläche entfernten Atomen zu einfallenden Partikeln bezeichnet

${ }^{45}$ channel (engl.): kristallographischer Kanal, in dem sich vergleichsweise lange lonentrajektorien ausbilden können, ohne dass es zu einem Streuprozess kommt
} 


\subsection{Plasmaeigenschaften}

Als Plasma wird ein teilweise oder vollständig ionisiertes aber quasineutrales Gas bezeichnet, das im Fall eines für die Schichtabscheidung benutzten Niederdruckplasmas aus sehr energiereichen, heißen Elektronen mit Energien von einigen $\mathrm{eV}$ und vergleichsweise kalten Ionen besteht. Die Elektronen besitzen wegen ihrer im Vergleich zu den Ionen deutlich geringeren Masse eine wesentlich höhere Mobilität; die Geschwindigkeitsverteilung der Elektronen lässt sich durch eine Maxwell-Boltzmann Verteilung beschreiben, die gegeben ist durch [Ger97]

$f(v) d v=\sqrt{2 / \pi} \cdot\left(m / k_{B} T\right)^{3 / 2} \cdot v^{2} \cdot \exp \left(-m v^{2} / 2 k_{B} T\right) d v$

wobei $\mathrm{k}_{\mathrm{B}}$ die Boltzmannkonstante, $\mathrm{T}$ die Temperatur, sowie $\mathrm{m}$ und $\mathrm{v}$ die Masse und Geschwindigkeit der Elektronen bezeichnen. Typische für die Schichtabscheidung benutzte Plasmadichten liegen zwischen $10^{9}$ und $10^{12} \mathrm{~cm}^{-3}$ [Fra94]. Im Plasma bildet sich aufgrund der stark unterschiedlichen Massen um die positiv ionisierten Gas-lonen eine Raumladung aus Elektronen, die das Ionen-Coulombpotential abschirmt. Diese Abschirmlänge wird als Debye-Länge $I_{D}$ bezeichnet und lässt sich mit der Elektronentemperatur $T$ und der Ladungsträgerdichte $n$ schreiben als

$l_{D}=\sqrt{\frac{\varepsilon_{0} k_{B} T}{n e^{2}}}$

Die Größen $\varepsilon_{0}, \mathrm{k}_{\mathrm{B}}$ und e bezeichnen die Dielektrizitätskonstante, die Boltzmannkonstante und die Elektronenladung. Als Konsequenz kann nur innerhalb einer Kugel vom Radius der Debye-Länge die Quasineutralität des Plasmas verletzt sein, typische Debye-Längen liegen im Bereich von etwa $10^{-4} \mathrm{~m}$. An den Rändern des Plasmas bildet sich aufgrund der asymmetrischen Beweglichkeit der Elektronen und Ionen und die dadurch bedingte höhere Elektronenverlustrate ein Potentialverlauf aus, wie er in Abb. 3.1 schematisch gezeigt ist [Pow99]. Die selbstständige Entladung wird durch die im elektrischen Feld beschleunigten Elektronen aufrecht erhalten, die durch Stoßionisation lawinenartig neue Gas-Ionen bilden. 


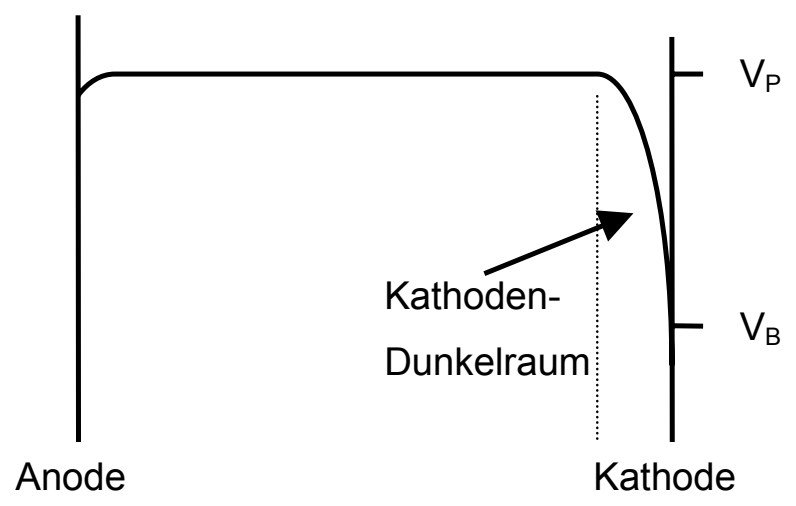

Abb. 3.1: Potentialverlauf als Funktion des Abstands zwischen Anode und Kathode, $V_{P}$ wird als Plasmapotential, $V_{B}$ als Bias-Spannung bezeichnet, schematisch nach [Pow99]

Die auftretende Potentialdifferenz an der Kathode, die im allgemeinen bei einigen $100 \mathrm{~V}$ liegt, bestimmt die Energie der auftreffenden Argon-Ionen und damit die Sputterausbeute. Für die reaktive Deposition beobachtet man generell eine Veränderung des Kathodenpotentials, wenn das Target mit der reaktiven Gaskomponente belegt wird, da sich dann eine Änderung in der Sekundärelektronenemission ergibt [Pow99]. Dieses Verhalten wird in Kap. 3.7 untersucht.

\subsection{Verwendete Sputteranlage}

Die zur reaktiven Sputterdeposition verwendete Anlage ist eine Endura 5500 von Applied Materials [Pow99]. Diese in Abb. 3.2 gezeigte Anlage ist eine modular aufgebaute Mehrkammer-UHV-Anlage, die für die Beschichtung von $150 \mathrm{~mm}$ Si Wafer ausgelegt ist. Sie besteht aus zwei Haupt- bzw. Transferkammern, welche durch eine Rücksputter- und eine Abkühlkammer verbunden sind. An den Hauptkammern sind die einzelnen Depositionskammern angeflanscht, zusätzlich existiert noch eine Vorheiz-Kammer, die zur Orientierung der Wafer und Entgasung von Substratadsorbaten vor der Schichtabscheidung benutzt wird. Die Wafer werden mittels mechanischer Greifarme aus den Schleusenkammern in die Hauptund Sputterkammern bewegt. An den Hauptkammern befinden sich Turbomolekularpumpen mit vorgeschalteten Vorpumpen, die einen Vakuumenddruck von etwa $p=1 \cdot 10^{-8}$ Torr erreichen. 16

${ }^{46}$ Im folgenden werden Druckangaben in Torr gemacht, da an der verwendeten PVD-Anlage standardmäßig die Druckeinheit Torr benutzt wird. Die Umrechnung in SI-Einheiten ist gegeben durch 1 bar $=1 \cdot 10^{5} \mathrm{~Pa}=760$ Torr . 
a)

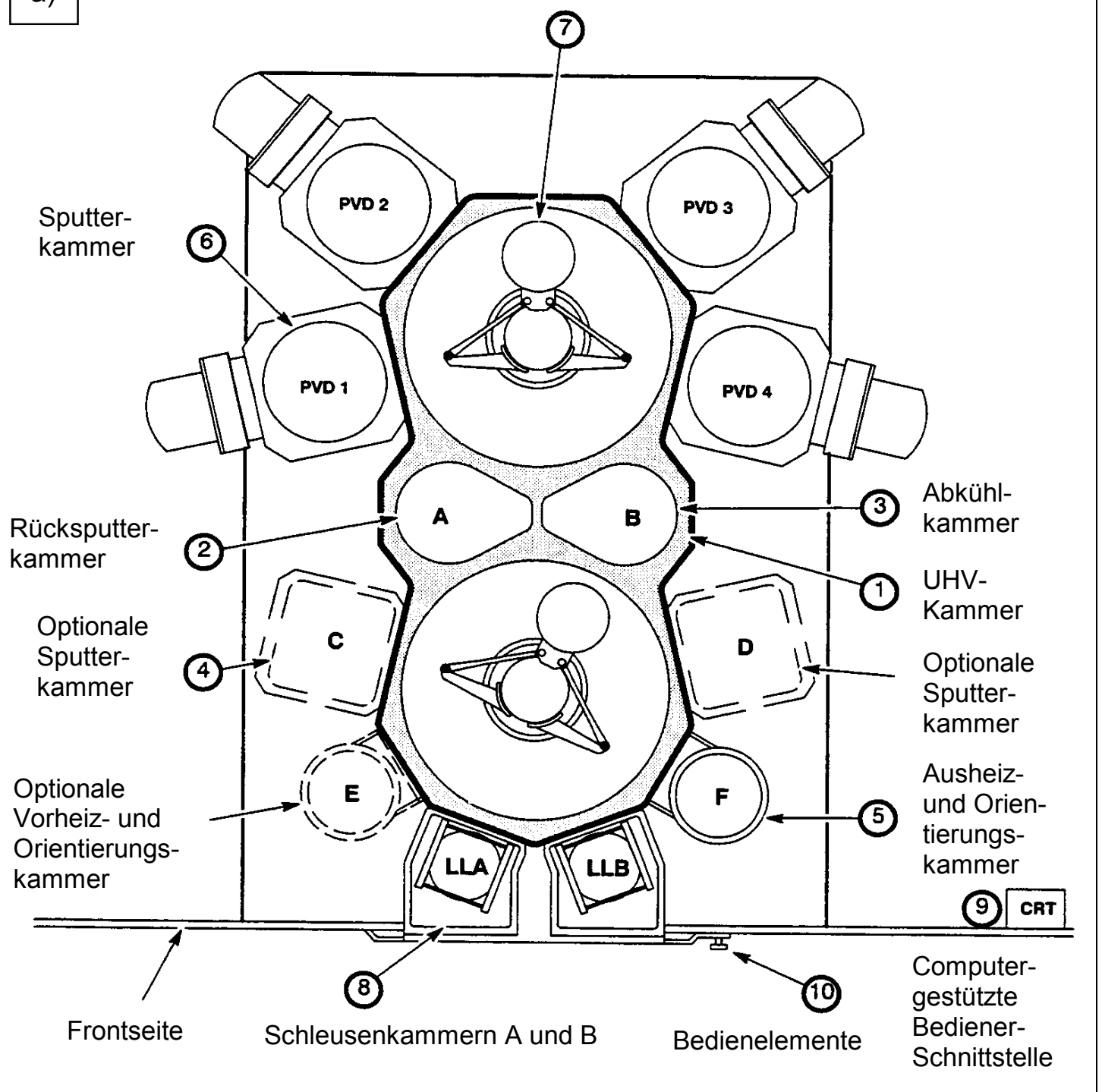

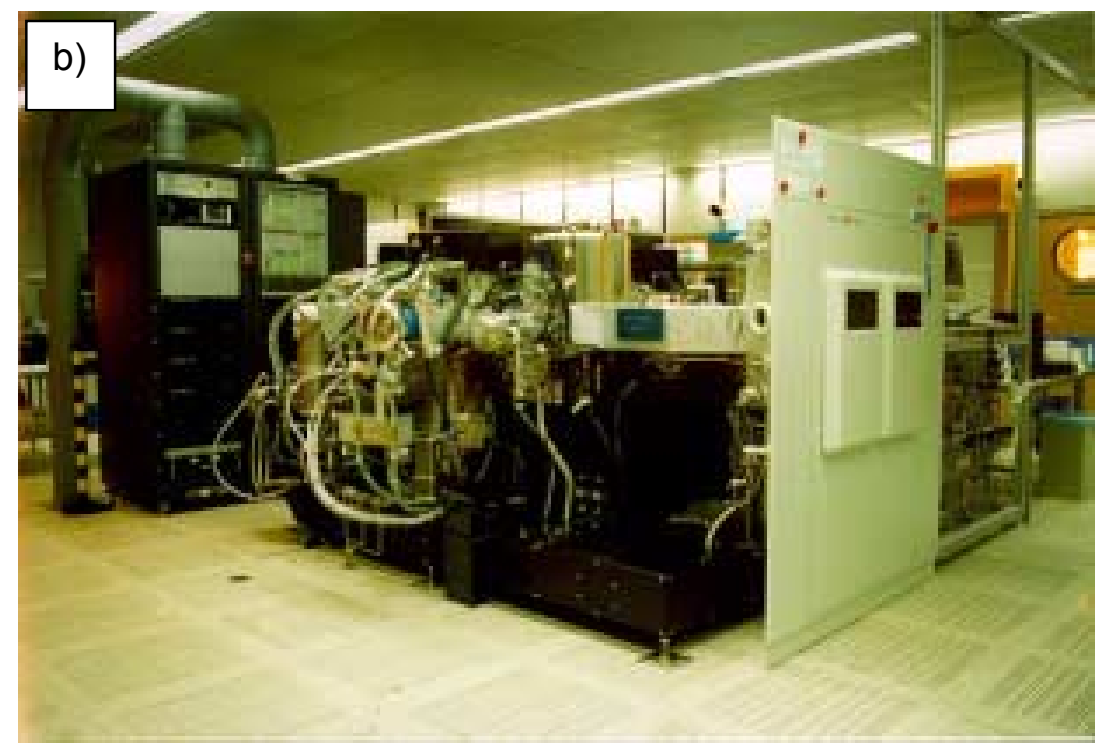

Abb. 3.2: a) Schematische Skizze der Endura 5500 (Applied Materials) nach [Pow99], b) Seitenansicht (Infineon Technologies AG, München) 
An jeder Sputterkammer ist neben einer Turbomolekularpumpe je eine Cryopumpe vom Typ Cryo-Torr $8^{47}$ angeflanscht, die zur Reduzierung des Wasserdampfrestgasdrucks eingesetzt wird. Während der Deposition pumpt die Cryopumpe als einzige Pumpe an der Depositionskammer und bestimmt somit die während der Deposition relevante Pump- bzw. Saugleistung $s_{p}$. Über ein vor die Cryopumpe vorgeschaltetes Drosselventil kann die Pumpleistung graduell verringert werden. Die Programmierung und Kontrolle der Deposition erfolgt interaktiv über eine vom Hersteller gelieferte Software. Details zu der verwendeten PVD-ClusterAnlage beschreibt Powell [Pow99]. Die hier genannte Sputter-Cluster-Anlage ermöglicht neben der wahlweise reaktiven Abscheidung von $\mathrm{Ir}$ und $\mathrm{IrO}_{2}$ Schichten, sequentiell verschiedene Schichten (z.B. Ti/TiN, Ta/TaN, TaSiN, Pt und Metallisierungen wie Al, Cu usw.) ohne Brechen des Vakuums zu deponieren und damit Grenzflächenverunreinigungen zu minimieren. Das in dieser Arbeit verwendete Iridium-Sputter-Target hat einen Durchmesser von $28,7 \mathrm{~cm}$, eine Dicke von $31,75 \mathrm{~mm}$ und eine vom Hersteller ${ }^{48}$ angegebene Reinheit von mindestens 99,8\%. Die Zerstäubung erfolgt magnetfeldunterstützt mit einem eine Flussdichte von $B=20 G=2 \cdot 10^{-3} T$ erzeugenden, rotierenden Permanentmagneten hinter dem Sputter-Target. Der Target-Substrat-Abstand wurde nach einer Optimierung der Schichtdickenhomogenität über einen $150 \mathrm{~mm}$ Wafer auf $d_{t-s}=50 \mathrm{~mm}$ eingestellt. Als Sputtergase wurden $5 \mathrm{~N}-$ Argon $^{49}$ bzw. $4 \mathrm{~N}-$ Sauerstoff eingesetzt [Pam01a].

\subsection{Depositionsparameter}

Die bereits in Kap. 1.4 diskutierten thermodynamischen Überlegungen und die bislang zur $\mathrm{IrO}_{2}$-Deposition erschienenen Veröffentlichungen legen den Schluss nahe, dass die Qualität bzw. die physikalischen Eigenschaften der $\mathrm{IrO}_{2}$ Schichten sehr empfindlich von den Depositionsparametern abhängig sind: Es wurde von Cho et al. [Cho97] und Cha et al. [Cha99] gezeigt, dass bereits geringe Änderungen der Sputterparameter nach Hochtemperaturbehandlungen zu einer morphologischen und chemischen Degradation durch Reduktion bzw. vorzeitiger Sublimation der $\mathrm{IrO}_{\mathrm{x}}$ Schichten führt. Aus diesem Grund soll die Optimierung der Deposition der Schichten hier genauer beleuchtet werden. Im folgenden werden die Depositionsparameter erläutert und es soll diskutiert werden, über welchen Bereich sie variiert werden können.

In der beschriebenen Anlage hat man die Möglichkeit, über drei an die Depositionskammer angeschlossene Gasflussregler den Argon-, den Argon-Wafer-Heizer-, und den Sauerstoff-

\footnotetext{
${ }^{47}$ CTI-Cryogenics, Helix Technology Corp.

48 Tosoh SMD, Inc., http://www.tosoh.com

${ }^{49} 5 \mathrm{~N}$ entspricht einer Reinheit von 99,999 Vol\%, 4N entsprechend einer Reinheit von 99,99 Vol\%
} 
fluss $\mathrm{Q}\left(\mathrm{O}_{2}\right)$ einzustellen. Dabei wurden SEC4400 Gasflussregler der Firma STEC ${ }^{50}$ verwendet, die mit einer vom Hersteller angegebenen Genauigkeit von maximal $\pm 1 \%$ Abweichung des geeichten Maximalflusses arbeiten. Dieser wird generell in Standardkubikzentimetern pro Minute (sccm) angegeben 51 . Die Umrechnung von den angegebenen Standardkubikzentimetern pro Minute in SI-Einheiten ist gegeben durch

$1 \mathrm{sccm}=\frac{1}{1000} l \cdot \frac{1 \mathrm{bar}}{60 \mathrm{~s}}=1, \overline{6} \cdot 10^{-5} \frac{\mathrm{bar} \cdot \mathrm{l}}{\mathrm{s}}=1, \overline{6} \frac{\mathrm{Pa} \cdot \mathrm{l}}{\mathrm{s}}=1, \overline{6} \cdot 10^{-3} \frac{\mathrm{Pa} \cdot \mathrm{m}^{3}}{\mathrm{~s}}$.

Der mögliche Arbeitsbereich der einzelnen Gasflussregler ist in Tab. 3.1 angegeben. Das Argon-Heizgas wird benutzt, um den Wafer vor der Schichtdeposition auf die gewünschte Temperatur aufzuheizen. Unter dem Wafer befindet sich ein Heizblock, durch die thermische Ankopplung des Wafers über das Heizgas wird das Substrat innerhalb von etwa 30 Sekunden auf die voreingestellte Temperatur des Heizblockes gebracht [Pow99]. Der ArHeizgasfluss wird nicht variiert, sondern es wird ein fester, bereits optimierter Ar-Fluss von $Q(\mathrm{ArH})=10 \mathrm{sccm}$ gewählt, um eine gute thermische Ankopplung und eine konstante Substrattemperatur sicherzustellen.

Tab. 3.1: Arbeitsbereich der verwendeten Gasflussregler

\begin{tabular}{llll}
\hline Gasflussregler & Argon & Argon Heizgas & Sauerstoff \\
\hline $\begin{array}{l}\text { geeichter Fluss- } \\
\text { bereich [sccm] }\end{array}$ & $0 . .280$ & $0 . .50$ & $0 . .100$ \\
\hline
\end{tabular}

Für die Deposition kann man mit einer Druckregelung arbeiten, bei der die Drosselventilstellung so angepasst wird, dass ein gewünschter Gesamtdruck während der Deposition über einen Regelmechanismus konstant gehalten wird. Für die hier beschriebene Form der Abscheidung wurde bewusst auf diese Möglichkeit verzichtet, und mit einer - neben dem Arund dem $\mathrm{O}_{2}$-Fluss als weiterer Sputter-Parameter fungierenden - definierten Saugleistung gearbeitet, um stationäre Prozesse untersuchen und auswerten zu können.

Als weiterer Parameter steht die ins Plasma eingekoppelte Leistung des DC-Generators ${ }^{\frac{52}{2}}$ bis zu 20 kW zur Verfügung, mit deren Hilfe der lonisationsgrad des Plasmas und die Sputterrate bestimmt werden kann. Um hinreichend hohe, aber trotzdem kontrollierbare Wachstums-

\footnotetext{
${ }^{50}$ http://www.horibastec.com

${ }^{51}$ Standard steht für thermodynamische Standardbedingungen, also $T=293 \mathrm{~K}$, und einen Referenzdruck von $p=1$ bar

${ }^{52}$ Advanced Energy, MDX-L6
} 
raten zu erzeugen, wurden hier systematisch Sputterleistungen zwischen $P=100 \mathrm{~W}$ und $1500 \mathrm{~W}$ untersucht. Schließlich wird die Abhängigkeit der $\mathrm{IrO}_{\mathrm{x}}$-Abscheidung von der Substrattemperatur untersucht. Das verwendete Sputtertool Endura bietet die Möglichkeit, Depositionstemperaturen von Raumtemperatur (RT) bis zu etwa $550^{\circ} \mathrm{C}$ zu wählen. Systematisch untersucht wurden Abscheidetemperaturen von $100^{\circ} \mathrm{C}, 300^{\circ} \mathrm{C}$ und $500^{\circ} \mathrm{C}$. Als niedrigste $\mathrm{Ab}-$ scheidetemperatur wurde bewusst nicht Raumtemperatur, sondern $100^{\circ} \mathrm{C}$ gewählt, um eine bessere Reproduzierbarkeit der Schichteigenschaften aufgrund der erhöhten Temperaturstabilität bei $100^{\circ} \mathrm{C}$ zu erreichen. Bei längerer Beschichtungszeit (bzw. hoher Waferanzahl) beobachtet man bei Raumtemperatur-Abscheidung eine durch das Plasma hervorgerufene Erhöhung der wirklichen Abscheidetemperatur [Nag01].

Die in Tab. 3.2 dargestellten, für die Deposition zur Verfügung stehenden Parameter und deren Einfluss auf den Abscheideprozess sollen in den folgenden Abschnitten mittels Analyse durch generische Plasmakurven diskutiert werden.

Tab. 3.2: Abscheideparameter für die $\mathrm{IrO}_{x}$ Deposition

\begin{tabular}{ll}
\hline Parameter & $\begin{array}{l}\text { Systematisch untersuchter } \\
\text { Bereich }\end{array}$ \\
\hline DC-Leistung $\mathbf{P}[\mathbf{k W}]$ & $100 . .1500$ \\
Systempumpen-Saugleistung $\mathbf{s}_{\mathbf{p}}[\mathbf{I} / \mathbf{s}]$ & $70 . .440$ \\
Depositionsdruck p [mTorr] & $2,5 . .20$ \\
$\mathbf{O}_{2}$ Partialdruck $\mathbf{p}\left(\mathrm{O}_{2}\right)$ [mTorr] & $0 . .15$ \\
Abscheidetemperatur $\mathbf{T}_{\mathbf{s}}\left[{ }^{\circ} \mathbf{C}\right]$ & $100 . .500$ \\
\hline
\end{tabular}

\subsection{Optimierung des Depositionsprozesses mittels generischer Kurven}

Um den reaktiven Sputterprozess zu charakterisieren und zu optimieren, nimmt man für die vorher gewählten Sputterparameter wie Saugleistung $s_{p}$, Sputterleistung P, Substrattemperatur $\mathrm{T}_{S}$ und Argonpartialdruck $\mathrm{p}(\mathrm{Ar})$ eine sogenannte generische Plasma-Kurve auf [Ros89], [Pow99], [Oka86]. Bei dieser Analysemethode untersucht man die Plasmadaten Kammerdruck, Target-Bias ${ }^{53}$ und lonenstrom in Abhängigkeit von der zugegebenen reaktiven Gasmenge vor und während der Deposition. Als geeignete Abszissen-Skalierung wählt man an-

\footnotetext{
${ }^{53}$ Die am Sputter-Target abfallende Spannung
} 
stelle des reaktiven Gasflusses den Partialdruck des reaktiven Gases, da dies die physikalisch relevante Größe ist, die die Schichtwachstumsprozesse während der Deposition bestimmt. Durch diese Abszissenskalierung wird ein Vergleich von Depositionseigenschaften auf unterschiedlichen Sputtersystemen ermöglicht, da nicht der reaktive Fluss $Q\left(\mathrm{O}_{2}\right)$ bzw. das in vielen Veröffentlichungen verwendete Flussverhältnis $Q\left(O_{2}\right) /\left(Q(A r)+Q\left(O_{2}\right)\right)$, sondern vielmehr der Partialdruck des reaktiven Gases als universelle Größe anzusehen ist.

Der Partialdruck des reaktiven Gases $\mathrm{p}\left(\mathrm{O}_{2}\right)$ ist nach dem bisher Gesagten einerseits durch den reaktiven Fluss $\mathrm{Q}\left(\mathrm{O}_{2}\right)$, andererseits durch die Pumpleistung der Systempumpe bestimmt. Während des Sputterns wird der Partialdruck aber auch noch durch den GetterEffekt verändert, d.h. durch Reaktion des reaktiven Gases mit den auf den Oberflächen kondensierenden Metallatomen. Die Gasphasenreaktion $\mathrm{Ir}+\mathrm{O}_{2} \rightarrow \mathrm{IrO}_{2}$ ist irrelevant, da ein für den Energieübertrag notwendiger 3-Teilchenstoß bei den analysierten Vakuum-Bedingungen statistisch unwahrscheinlich ist [Beh91]. Eine chemische Reaktion am Sputter-Target, die über eine Physisorption hinausgeht, ist sehr unwahrscheinlich, da eine signifikante Chemisorption von Sauerstoff auf Iridium-Oberflächen erst bei Temperaturen von deutlich über $550^{\circ} \mathrm{C}$ beobachtet wurde [Gme78].

Der Getter-Effekt während der reaktiven Sputterdeposition ist in der Literatur ausführlich untersucht und diskutiert worden [Zhu98]. Die Vielzahl der mathematischen Beschreibungen basiert auf der folgenden Differentialgleichung für den Druck $p(t)$

$$
\frac{d p(t)}{d t}=\frac{1}{V_{c h}}\left(\sum_{i} Q_{i}^{i n}-Q_{t}^{a d}+Q_{t}^{d e s}-Q_{s}-p(t) \cdot s_{p}\right)
$$

Dabei ist $Q_{i}^{i n}$ der über den Gasflussregler i in die Kammer eingelassene Gasfluss, $Q_{t}^{a d}$ der am Sputter-Target adsorbierte Gasfluss, $Q_{t}^{\text {des }}$ der vom Target während des Sputterprozesses in den Gasraum emittierte Gasfluss und $Q_{s}$ der Gasfluss, welcher bei der Kondensation des gesputterten Materials auf dem Substrat und an den Kammerwänden gegettert wird. $V_{c h}$ stellt das Kammervolumen und $s_{p}$ die Saugleistung der Vakuumpumpe dar. Die Flüsse sind schematisch in Abb. 3.3 dargestellt. 


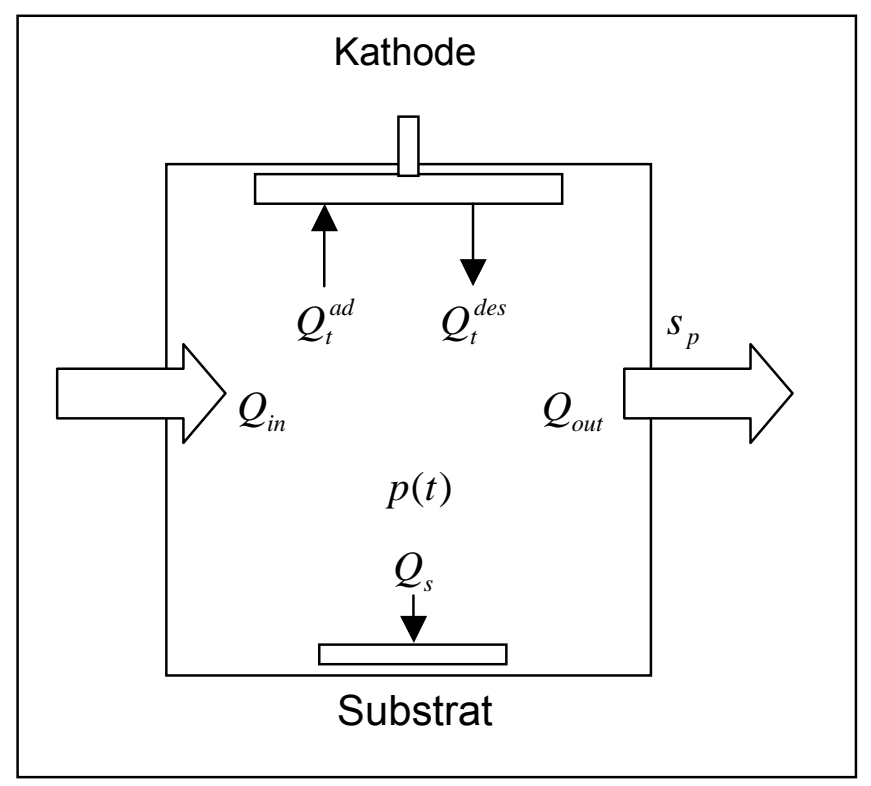

Abb. 3.3: Diagramm zu den in der Sputterkammer diskutierten Gasflüssen

Allgemein ist der Gasfluss $Q$ dem Massenstrom $\dot{m}$ bzw. dem Teilchenstrom $\dot{v}$ proportional, der sich unter der Voraussetzung konstanter Temperatur T aus der allgemeinen Gasgleichung

$Q \equiv p \cdot \dot{V}=\dot{m} \cdot \frac{R \cdot T}{M}=j_{m} \cdot A \cdot \frac{R \cdot T}{M}=\dot{V} \cdot R \cdot T$

mit den für die Gasgleichung üblichen Bezeichnungskonventionen ableiten lässt [Wut88]. Darüber hinaus stellt $j_{m}$ die Massenstromdichte durch die Querschnittsfläche $A$ dar. Im stationären Fall ist nach Gleichung (3.3) das Kammervolumen irrelevant und es stellt sich am Sputter-Target ein Gleichgewicht der Gas-Adsorptions- und Desorptionsrate ein, so dass gilt [Kus90]

$Q_{t}^{a d}=Q_{t}^{d e s}$

Aus Gleichung (3.3) folgt, dass $Q_{s}$ den effektiven Verbrauch des Reaktivgases durch den Getter-Effekt während der reaktiven Deposition darstellt und für $p_{s p}$ als stationärem Druck während der Deposition die folgende Gleichung gelten muss

$\sum_{i} Q_{i}^{i n}=p_{s p} \cdot s_{p}+Q_{s}$

Anhand des Vergleiches mit der stationären Situation vor der Deposition

$\sum_{i} Q_{i}^{i n}=p_{p} \cdot s_{p}$ 
mit $p_{p}$ als Gleichgewichts-Spüldruck, ergibt sich

$$
\begin{aligned}
& p_{p} \cdot s_{p}=p_{s p} \cdot s_{p}+Q_{s} \\
& \Leftrightarrow Q_{s}=s_{p}\left(p_{p}-p_{s p}\right) \equiv s_{p} \cdot \Delta p
\end{aligned}
$$

Mithilfe dieser Gleichung lässt sich, unter der Annahme, dass kein Ar-Sputtergas verbraucht wird ${ }^{6}$, der reaktive Gasverbrauch $Q_{s}$ durch den Getter-Effekt während der Deposition quantitativ erfassen. Diese Methode wurde für die Optimierung der reaktiven Sputter-Deposition der $\mathrm{IrO}_{2}$ Barrierenschichten benutzt. 55

\subsubsection{Einfluss der Pumpleistung}

Die verwendete Cryopumpe Cryo-Torr 8 hat im ungedrosselten Betrieb nach Herstellerangaben eine theoretische Pumpleistung für Argon von $s_{p}(A r)=1200 \mathrm{l} / \mathrm{s}$, diese wird aber aufgrund der Flansch-Konduktivität $c_{p}^{56}$ auf die sogenannte effektive Pumpleistung $s_{p, \text { eff }}$ reduziert. Diese Größen addieren sich reziprok gemäß [Wut88]

$\frac{1}{s_{p}}=\frac{1}{s_{p, t h}}+\frac{1}{c_{p}}$

Um die effektive Pumpleistung, die sich auf reines Argon bezieht, zu bestimmen, wurde in die auf den Basisdruck von $p=1 \cdot 10^{-8}$ Torr evakuierte Kammer ein wohldefinierter Argongasfluss eingelassen. Die Pumpgeschwindigkeit, die im allgemeinen in I/s angegeben wird, lässt sich, wenn sich im Rezipienten ein stationäres Druckgleichgewicht eingestellt hat, nach der aus Gleichung (3.3) folgenden Formel berechnen

$$
s_{p}=\frac{Q_{A r}}{p_{s t a t}}
$$

Für die effektive Argon-Pumpleistung der verwendeten Cryopumpe ergibt sich ein Wert von $\mathrm{s}_{\mathrm{p}, 1}=440 \mathrm{l} / \mathrm{s}$, mit einem in die Pumpe integrierten Drosselventil kann die Konduktivität und damit die effektive Pumpleistung graduell variiert werden. Untersucht wurden generische Kurven bei der maximalen effektiven Pumpleistung, bei einer reduzierten Pumpleistung $s_{p, 2}=200 \mathrm{l} / \mathrm{s}$ und einer deutlich kleineren Pumpleistung von $s_{p, 3}=70 \mathrm{l} / \mathrm{s}$. Die Pumpgeschwindigkeit $s_{p}$ ist abhängig von der Gasart und eine Funktion des Gasdrucks. Für das im folgenden erläuterte Optimierungsverfahren wird aber nur ein sehr kleiner Druckbereich untersucht,

\footnotetext{
${ }^{54}$ Sicherlich gerechtfertigt, da RBS-Messungen an den Schichten einen Ar-Gehalt in der Größenordnung von $1 \%$ ergeben

${ }^{55}$ Deutsches Patent 1-01-10681.5 vom 6.3.2001

${ }^{56}$ Flansch-Konduktivität: conductance of port (engl.)
} 
für den die Saugleistung der verwendeten Cryopumpe als konstant angenommen werden kann [Wut88]. Die Abhängigkeit von der Gasart soll im folgenden noch diskutiert werden.

Man erkennt in Abb. 3.4 die lineare Zunahme des Sauerstoffpartialdrucks mit dem reaktiven Fluss, entsprechend der Gleichung $p_{\text {ges }}=1 / s_{p} \cdot Q\left(O_{2}\right)+p_{A r}$. Durch die hohe Pumpleistung $\mathrm{s}_{\mathrm{p}, 1} \mathrm{im}$ ungedrosselten Modus wird nur ein Sauerstoffpartialdruck von etwa 2,5 mTorr bei maximalem Fluss $Q\left(\mathrm{O}_{2}\right)=100 \mathrm{sccm}$ erreicht, während bei der gedrosselten Pumpleistung $\mathrm{s}_{\mathrm{p}, 3}$ mit dem gleichen Fluss ein maximaler $\mathrm{O}_{2}$-Partialdruck von etwa 15 mTorr eingestellt werden kann, wie in Abb. 3.4 gezeigt ist. Der Nachteil in diesem Modus ist allerdings, dass durch die auf $\Delta Q=1 \mathrm{sccm}$ limitierte Genauigkeit des Gasflussreglers eine Feineinstellung des Sauerstoffpartialdrucks nur begrenzt möglich ist. Um detailliert die Veränderung der physikalischen Eigenschaften bei minimalen Sauerstoffpartialdruckvariationen zu analysieren, bietet sich daher der ungedrosselte Modus an.

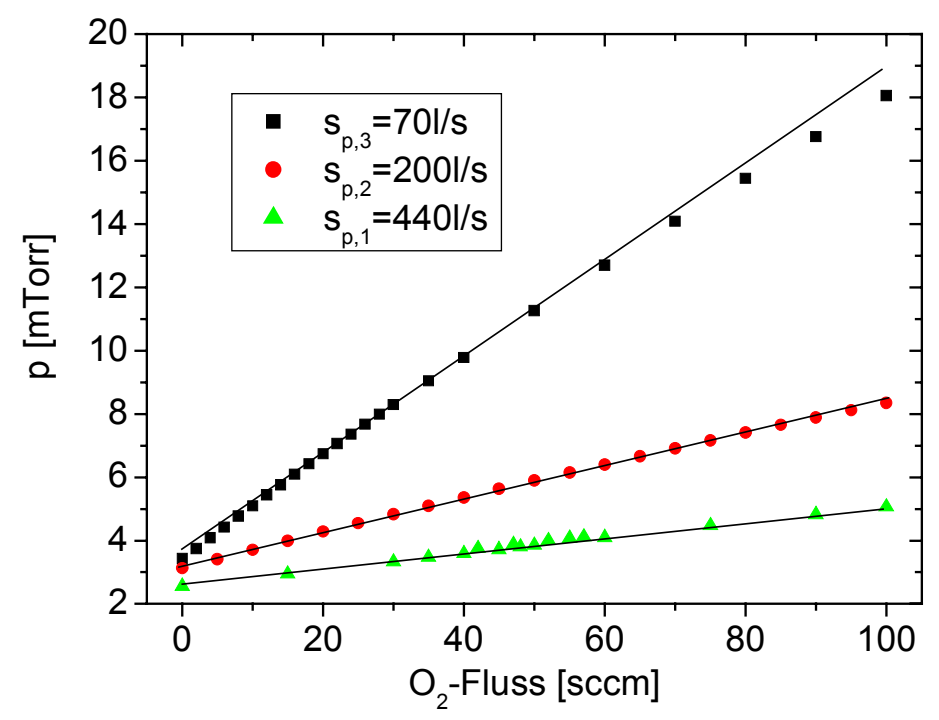

Abb. 3.4: Darstellung des gemessenen Gesamtdrucks $p$, der sich additiv aus dem Argondruck $p(\operatorname{Ar})$ und dem Sauerstoffpartialdruck $p\left(\mathrm{O}_{2}\right)$ zusammensetzt, in Abhängigkeit des reaktiven $\mathrm{O}_{2}$ Flusses bei verschiedenen Systempumpen-Saugleistungen $s_{p, 1}, s_{p, 2}$ und $s_{p, 3}$

Für die drei genannten Pumpleistungen sind generische Graphen für eine Leistung von $\mathrm{P}=300 \mathrm{~W}$ und bei einer Substrattemperatur von $\mathrm{T}_{\mathrm{S}}=100^{\circ} \mathrm{C}$ aufgenommen worden und in Abb. 3.5 dargestellt. Dabei wurde der Ar-Fluss $Q(A r)$ für die reduzierten Pumpleistungen so angepasst, dass sich für alle drei Bedingungen ein nahezu gleicher Ar-Partialdruck $\mathrm{p}_{\mathrm{Ar}}$ in der Kammer einstellt. 
a)

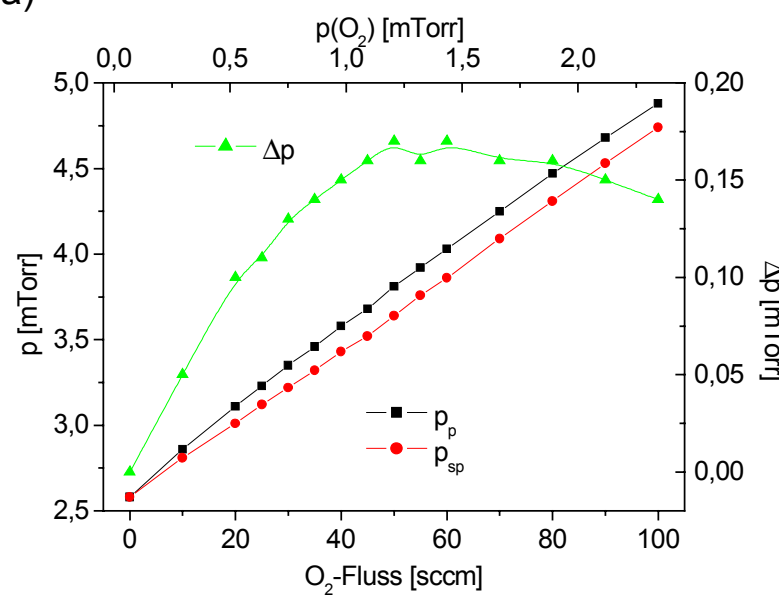

b)

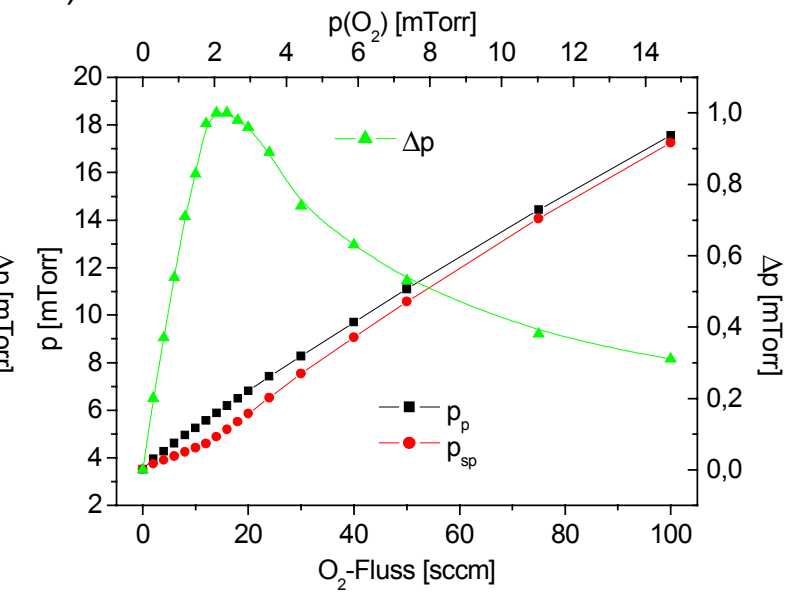

Abb. 3.5: Generische Graphen a) für $s_{p, 1}=440 \mathrm{l} / \mathrm{s}$ und b) für $s_{p, 3}=70 \mathrm{l} / \mathrm{s}, P=300 \mathrm{~W}, T_{S}=100^{\circ} \mathrm{C}$. Die grüne Kurve stellt direkt die Differenz des Spüldrucks $p_{p}$ und des Sputterdrucks $p_{s p}$ dar und kann als Gettereffekt während der Ir-Deposition gedeutet werden (s. Gl. 3.8)]

In Abb. 3.5 sind zusätzlich zu den stationären Spüldruckwerten $p_{p}$ die Gleichgewichtsdruckwerte während der Deposition $p_{s p}$ und die aus diesen Werten ermittelte Druckdifferenz $\Delta p$ gezeigt. Man erkennt anhand des generischen Graphen, der bei einer Pumpleistung von $70 \mathrm{l} / \mathrm{s}$ aufgenommen wurde, dass es einen deutlichen Knickpunkt im Verlauf des Sputterdrucks $p_{\mathrm{sp}}$ bei einem kritischen Sauerstoffpartialdruck von etwa 2 mTorr gibt. Bei dem Graphen, der bei einer Pumpleistung von $s_{p}=440 \mathrm{l} / \mathrm{s}$ aufgenommen wurde, ist kein evidenter Übergangspunkt nachweisbar. Der Knickpunkt markiert einen Wechsel im Sputterregime, denn anhand des schnell ansteigenden Gesamtdrucks jenseits des Übergangspunkts setzt eine Sättigung des Getter-Effektes des gesputterten Materials ein. Der Exzess-Sauerstoff führt zu einer oberflächlichen Belegung des Sputter-Targets mit Sauerstoffmolekülen. Es

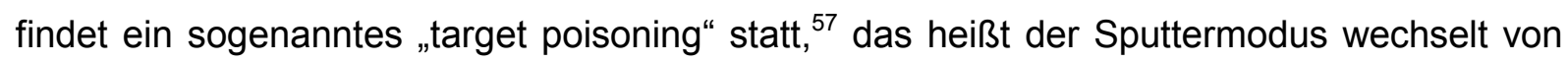
Metall-Target auf Metall-Sauerstoffadsorbat-Target. In diesem Modus wird der Sputteryield des Ir-Metalls mit zunehmendem Reaktivgaspartialdruck erheblich erniedrigt, da die Adsorptionsrate der reaktiven Gaskomponente stark zunimmt. Folglich wird durch die Ar-Ionen bei erhöhtem Partialdruck des reaktiven Gases zunehmend die Adsorbatschicht anstelle des Ir abgesputtert [Smi95], [Pow99]. Die in Abb. 3.6 gezeigten Target-Bias Graphen in Abhängigkeit des reaktiven Sauerstoffflusses weisen tatsächlich die in Kap. 3.4 diskutierte Abnahme des Target-Potentials auf, was das oben diskutierte Modell von der Target-Belegung bestätigt.

\footnotetext{
${ }^{57}$ target poisoning (engl.): Verunreinigung des Sputtertargets durch Adsorption von reaktivem Sputtergas
} 


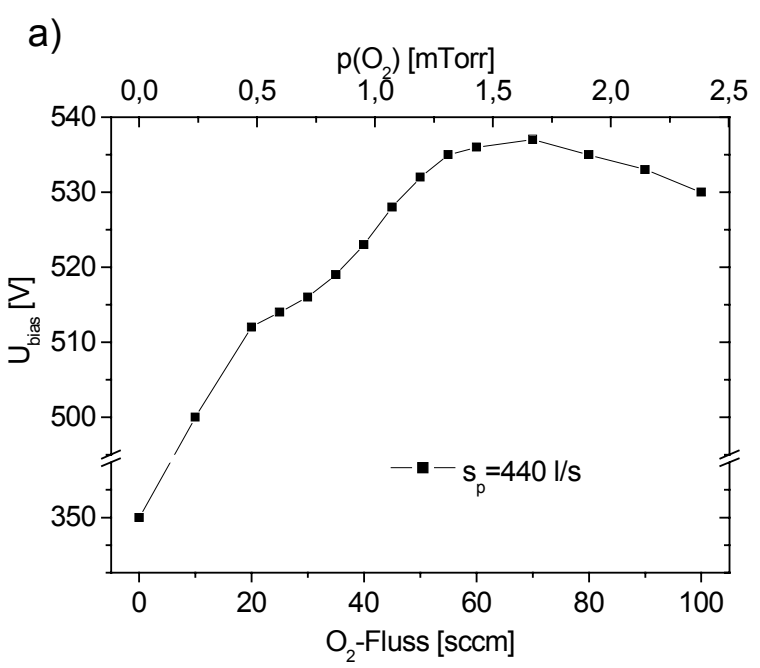

b)

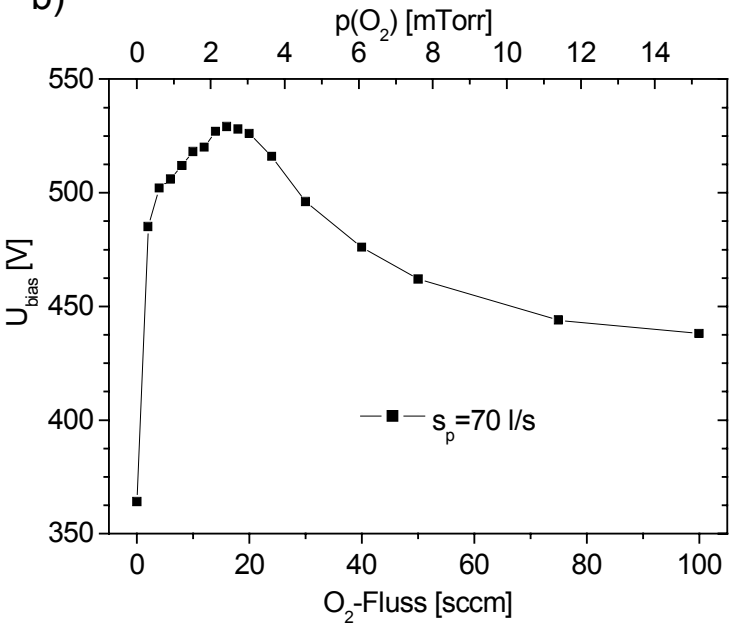

Abb. 3.6: Target-Potential in Abhängigkeit des reaktiven Sauerstoffflusses für a) $s_{p, 1}=440 \mathrm{l} / \mathrm{s}$ und für $b$ ) $s_{p, 3}=70 \mathrm{l} / \mathrm{s}, P=300 \mathrm{~W}, \mathrm{~T}_{\mathrm{s}}=100^{\circ} \mathrm{C}$. Die Abnahme des Target-Potentials beträgt in b) bei sehr hohen $\mathrm{O}_{2}$-Partialdrücken über $90 \mathrm{~V}$.

Im ungedrosselten Modus ist die Abnahme sehr gering und liegt im Bereich von einigen Volt, im gedrosselten Modus ist der Effekt bei hohen $\mathrm{O}_{2}$-Partialdrücken wie erwartet deutlicher ausgeprägt, es zeigt sich eine maximale Differenz von über $90 \mathrm{~V}$. Es soll in diesem Zusammenhang darauf hingewiesen werden, dass auch für die geringste untersuchte Saugleistung $\mathrm{s}_{\mathrm{p}, 3}=70 \mathrm{l} / \mathrm{s}$ bei den generischen Kurven kein Hystereseverhalten gefunden wurde, das heißt, der Verlauf der $\mathrm{p}_{\mathrm{sp}}$-Kurve ist unabhängig von der Tatsache, ob man von niedrigen zu hohen Sauerstoffpartialdrücken geht oder umgekehrt.

a)

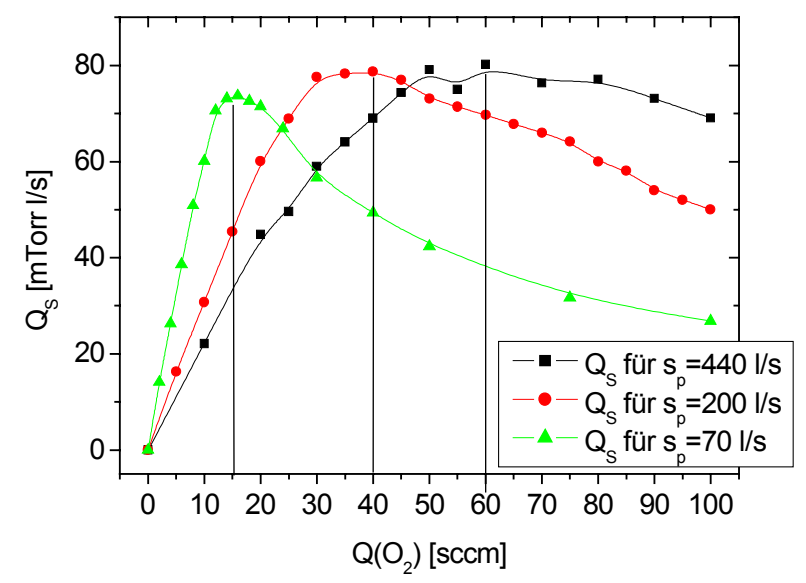

b)

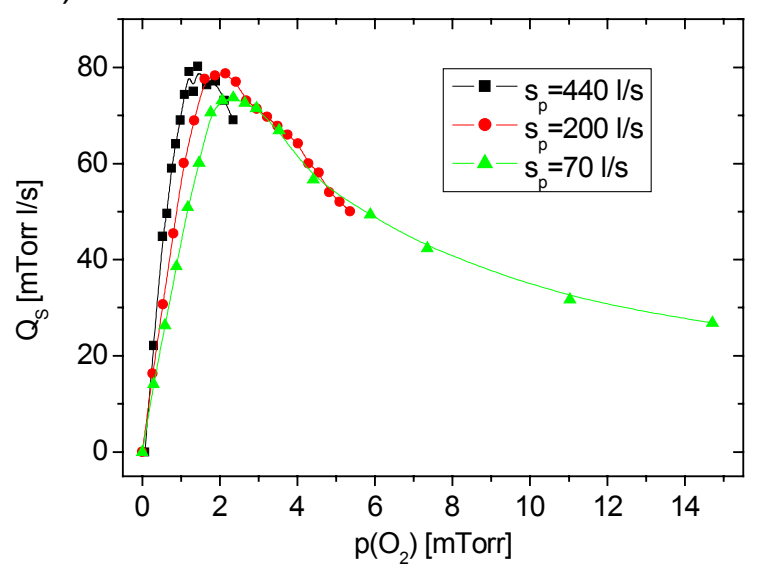

Abb. 3.7: a) Auftragung des gegetterten Sauerstoffflusses gegen a) den eingelassenen $\mathrm{O}_{2}$-Fluss, bzw. b) gegen den sich einstellenden $\mathrm{O}_{2}$-Partialdruck zur Optimierung der reaktiven Deposition, $P=300 \mathrm{~W}, \mathrm{~T}_{\mathrm{S}}=100^{\circ} \mathrm{C}$. Die zu den maximalen Sauerstoffgetterflüssen gehörenden $\mathrm{O}_{2}$-Flüsse sind gekennzeichnet.

Ein Hystereseverhalten wird sonst generell beim reaktiven Sputtern für sehr niedrige Pumpleistungen und Argonpartialdrücke gefunden, z.B. bei der von Wang et al. [Wan96] unter- 
suchten $\mathrm{RuO}_{\mathrm{x}}$-Deposition oder bei der von Okamoto et al. [Oka86] analysierten $\mathrm{SiN}_{\mathrm{x}^{-}}$ Deposition. Eine niedrige Pumpleistung erhöht die Adsorptionsrate am Target bei hohem Reaktivgaspartialdruck, ein geringer Ar-Partialdruck bewirkt eine Reduktion der Sputterrate. Die Auswertung der generischen Kurven mittels Gleichung (3.8) liefert das in Abb. 3.7 gezeigte $Q_{S}-Q\left(\mathrm{O}_{2}\right)$ bzw. $Q_{S}-p\left(O_{2}\right)$-Diagramm. Bei den in Abb. 3.7 gezeigten Kurven ist die gegetterte Flussmenge $Q_{S}$ gegen den reaktiven Sauerstofffluss $Q\left(\mathrm{O}_{2}\right)$ bzw. den Sauerstoffpartialdruck $p\left(\mathrm{O}_{2}\right)$ aufgetragen. Für geringe Sauerstoffpartialdrücke ist ein nahezu linearer Anstieg der Sauerstoffeinbaurate sichtbar, bei dem der vorgegebene Sauerstoffpartialdruck die limitierende Größe für den reaktiven Einbau darstellt. Der lineare Anstieg geht in eine Sättigung über, die maximale Sauerstoff-Integrationsrate ist bei einem für die Kammergeometrie und Sputterrate typischen $\mathrm{O}_{2}$-Partialdruck gegeben. Die Target-Belegung mit Sauerstoff ist durch ein dynamisches Gleichgewicht von der Sputterrate, also der Sputterleistung und dem Restgassauerstoffpartialdruck, der nicht gegettert wird, bestimmt. Das in Abb. 3.7 b) mit abnehmender Pumpleistung zu etwas größeren $\mathrm{O}_{2}$-Partialdrücken verschobene Maximum ist dadurch zu erklären, dass nach Gleichung (3.8) bei einer niedrigen Saugleistung $\mathrm{s}_{\mathrm{p}}$ für die gleiche Getterrate $\mathrm{Q}_{\mathrm{S}}$ eine höhere Druckdifferenz $\Delta p=p_{p}-p_{s p}$ beobachtet wird. Die Target-Belegung tritt somit bei einem Restgassauerstoffpartialdruck von etwa 1,3-1,5 mTorr konsistent für alle drei Saugleistungen $s_{p}$ bei einer Sputterleistung von $\mathrm{P}=300 \mathrm{~W}$ auf. Es ist anhand Abb. 3.7 a) nachweisbar, dass der Flussbereich, den man für die maximale Sauerstoffintegration benutzen kann, für geringere Pumpleistungen deutlich schmaler wird. Für sehr hohe Sauerstoffpartialdrücke jenseits des Übergangspunktes ist eine Abnahme des Getter-Prozesses beobachtbar, der hier aufgrund deutlich reduzierter Haftkoeffizienten der Sauerstoffmoleküle an bereits stöchiometrisch abgesättigten $\mathrm{IrO}_{2}$ Oberflächen der aufwachsenden Schicht vermutet wird. Der Vorteil der reaktiven Deposition bei maximaler Systempumpen-Saugleistung ist folglich eine deutlich erhöhte Genauigkeit und Reproduzierbarkeit des Partialdrucks des reaktiven Gases und damit der Schichteigenschaften im Gegensatz zu einer reduzierten Pumpleistung. Im stark reduzierten Pumpmodus haben nach Gleichung (3.10) kleine Abweichungen im reaktiven Fluss $\mathrm{Q}\left(\mathrm{O}_{2}\right)$ starke Auswirkungen auf den Reaktivgas-Partialdruck und damit im allgemeinen an dem kritischen Übergangspunkt auch auf die Schichteigenschaften.

\subsubsection{Temperaturabhängigkeit der generischen Kurven}

Die Substrattemperatur stellt einen sehr wichtigen Parameter für die Schichtdeposition dar, weil über diese die Mikrostruktur und die Morphologie deponierter Sputterschichten maßgeblich beeinflusst wird [Tho74], [Tho75]. Die bei Substrattemperaturen von $100^{\circ} \mathrm{C}-500^{\circ} \mathrm{C}$ aufgenommenen Sauerstoff-Getter-Flüsse $Q_{S}$ sind in Abb. 3.8 gezeigt. Die Skalierung der Ach- 
sen in Abb. 3.8 ist wie in der vorigen Abb. 3.7 gewählt. Man erkennt, dass die maximale Sauerstoffaufnahmerate $Q_{\mathrm{S} \text {,max }}$ unabhängig von der Substrattemperatur bei einem Sauerstoffpartialdruck $\mathrm{p}\left(\mathrm{O}_{2}\right)$ von 1,6 mTorr erreicht wird. Für die analysierten Bedingungen ist der absolute Wert von $Q_{S}$ am Maximum ebenfalls nicht signifikant temperaturabhängig und beträgt $Q_{S, \max }=80 \mathrm{mTorr}$ 1/s. Die errechneten Werte streuen aufgrund der limitierten Genauigkeit der kapazitiven Baratron-Drucksonde von etwa $\delta p=0,01 \mathrm{mTorr}$ um $\delta Q_{S}=4,5 \mathrm{mTorr} \mathrm{l} / \mathrm{s}$. Aus Übersichtsgründen sind die y-Fehlerbalken nicht mit eingezeichnet.

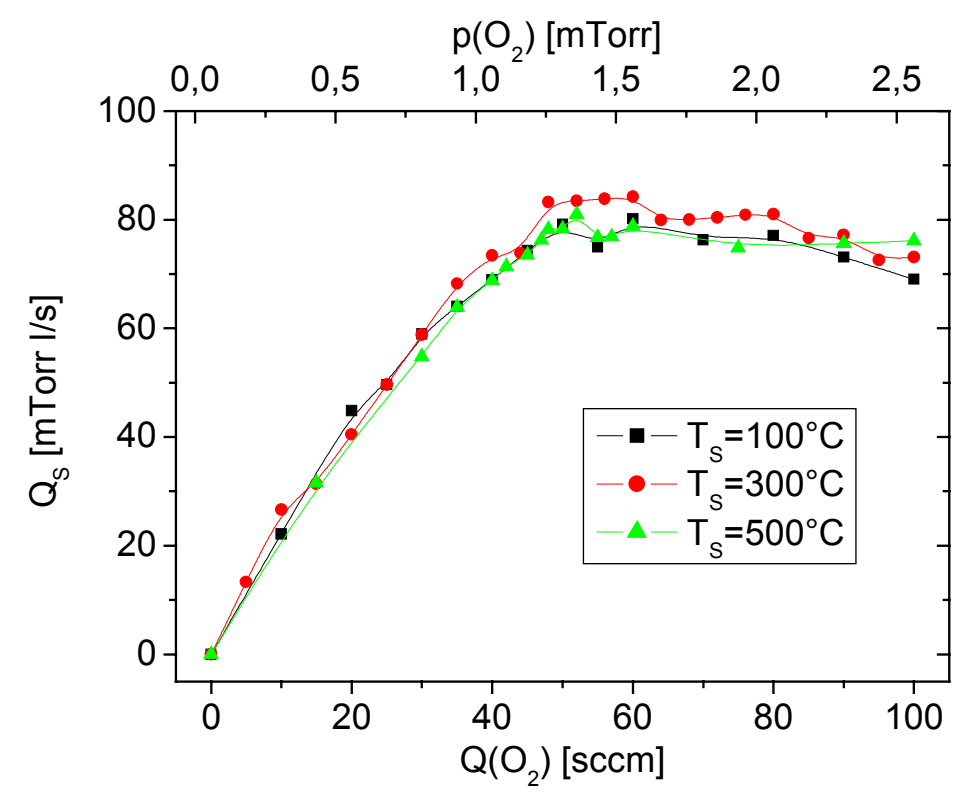

Abb. 3.8: Darstellung der gegetterten Flussmenge $Q_{S}$ in Abhängigkeit der eingelassenen Flussmenge $Q\left(\mathrm{O}_{2}\right)$ bzw. des sich einstellenden Reaktivgaspartialdrucks $p\left(\mathrm{O}_{2}\right)$ für verschiedene Substrattemperaturen $\mathrm{T}_{\mathrm{S}}, \mathrm{P}=300 \mathrm{~W}, \mathrm{~s}_{\mathrm{p}}=440 \mathrm{l} / \mathrm{s}, \mathrm{p}(\mathrm{Ar})=2,5 \mathrm{mTorr}$

Dieses Ergebnis deutet darauf hin, dass eine mit der Substrattemperatur verbundene Veränderung der Oberflächendiffusion und damit der Oberflächenmorphologie, die die effektive Getterfläche bestimmt [Wut88], keinen signifikanten Einfluss auf den Getter-Prozess nimmt. Vielmehr lässt sich aufgrund des temperaturunabhängigen Getter-Verhaltens schließen, dass die Adsorptionsenergie der Sauerstoffatome auf der Ir Schicht so groß ist, dass eine Reevaporation von Sauerstoffatomen bzw. Molekülen für die reaktive Deposition im untersuchten Temperaturintervall nicht relevant ist. Diese Ergebnisse sind konsistent mit Messungen von Ageev et al. [Age72], bei denen keine Temperaturabhängigkeit der Sauerstoffadsorption an Ir-Oberflächen zwischen 300 und $900 \mathrm{~K}$ festgestellt wurde.

Ob es sich bei dem Sorptionsprozess um eine Absorption, also eine Lösung des Sauerstoffs im kondensierten Iridium, oder um eine chemische Reaktion handelt, wird in Kap.4.3 analysiert. 


\subsubsection{Untersuchung der Sputterleistung mittels generischer Kurven}

Es wurde der Einfluss der in das Plasma eingekoppelten Leistung sowohl bei ungedrosselter Systempumpleistung von $\mathrm{s}_{\mathrm{p}, 1}=440 \mathrm{l} / \mathrm{s}$ als auch bei stark gedrosselter Pumpleistung von $\mathrm{s}_{\mathrm{p}, 3}=70 \mathrm{l} / \mathrm{s}$ untersucht. Die sich ergebenden generischen Kurven bzw. die aus den Daten errechneten Sauerstoffgetterflüsse sind in der folgenden Darstellung gezeigt. Man sieht anhand der in Abb. 3.9 gezeigten $Q_{S}-p\left(O_{2}\right)$-Diagramme bei der reduzierten Systempumpleistung für alle Sputterleistungen den Übergang in den oxidierten Target-Modus. Dieser tritt bei mit der Leistung nahezu linear zunehmenden Sauerstoffpartialdrücken auf. Dieser experimentelle Befund lässt sich dadurch deuten, dass in dem untersuchten Intervall der Sputteryield $Y_{\text {Ir }}$ nahezu linear mit der Sputterleistung zunimmt und damit proportional mehr Ir auf dem Substrat kondensiert. Für den präferentiell zu wählenden Modus mit ungedrosselter Pumpleistung erscheint eine Sputterleistung von $\mathrm{P}=300 \mathrm{~W}$ als geeignet, da für die Leistung von $\mathrm{P}=100 \mathrm{~W}$ die Ungenauigkeit aufgrund der geringen Sputter- und Getter-Rate zu hoch ist und für die Sputterleistung von $\mathrm{P}=500 \mathrm{~W}$ der zu untersuchende Übergangsbereich bei einem Sauerstofffluss von $\mathrm{Q}\left(\mathrm{O}_{2}\right)=90 \mathrm{sccm}$ an der oberen Regel-Grenze des SauerstoffGasflussreglers liegt.
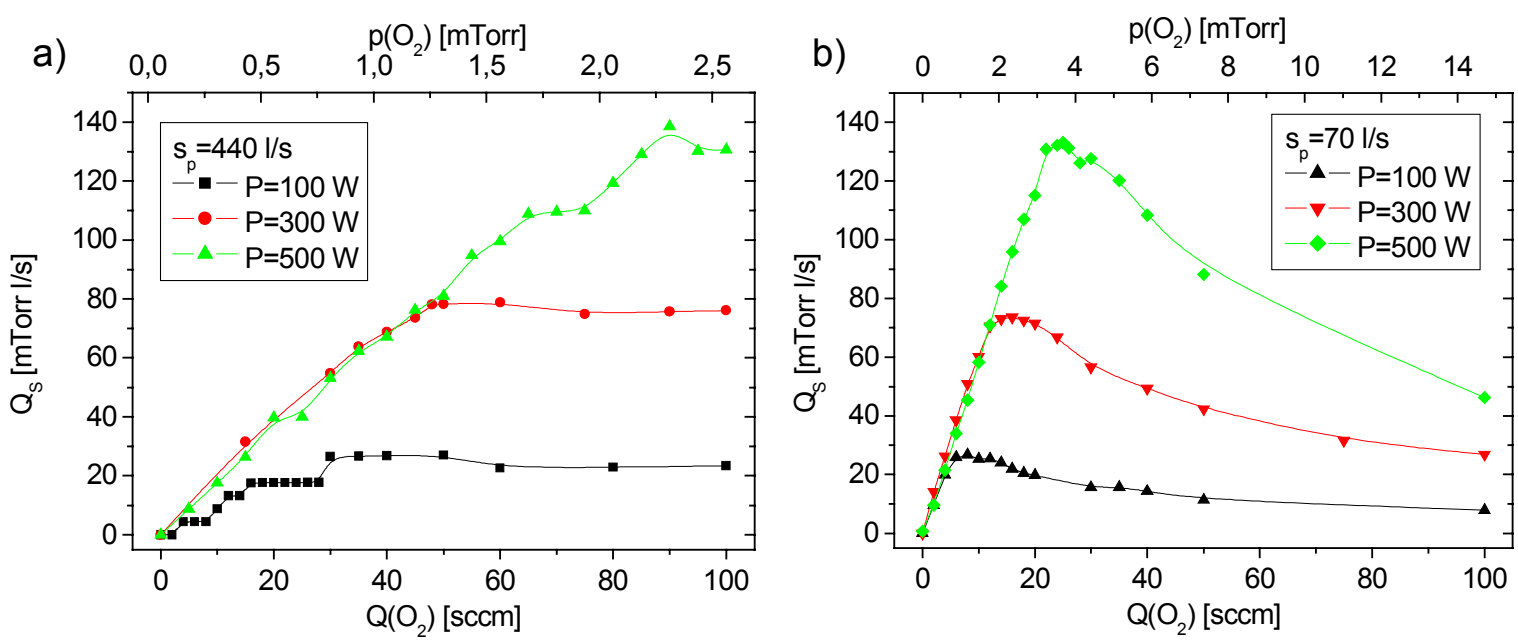

Abb. 3.9: Einfluss der Sputterleistung auf die gegetterte Flussmenge $Q_{S}$ bei einer gewählten Substrattemperatur von $T_{S}=100^{\circ} \mathrm{C}$; a) für die maximale Saugleistung $s_{p, 1}=440 \mathrm{l} / \mathrm{s}$ und b) für die reduzierte Saugleistung von $s_{p, 3}=70 \mathrm{l} / \mathrm{s}$. Dargestellt sind die $Q_{\mathrm{s}}$-Graphen für Sputterleistungen von $100 \mathrm{~W}, 300 \mathrm{~W}$ und $500 \mathrm{~W}$

\subsubsection{Einfluss des Argon-Partialdrucks auf die generischen Kurven}

Als weiterer Parameter, der Einfluss auf die Deposition nehmen kann, wurde neben dem Sauerstoff-Partialdruck auch der Argon-Partialdruck variiert. Der Druck des Argon-Edelgases entscheidet über die freie Weglänge der gesputterten Atome und beeinflusst somit besonders bei großen Target-Substrat-Abständen deren Streuung auf dem Weg zum Substrat. Mit 
zunehmendem Sputterdruck erwartet man einen Übergang von der ballistischen zur diffusiven Deposition und damit im allgemeinen auch eine Veränderung der Schichteigenschaften. Um den Einfluss des Ar-Partialdrucks zu untersuchen, wurden generische Kurven bei ungedrosselter Pumpleistung aufgenommen, bei denen der Ar-Fluss von 80 auf 160 und $240 \mathrm{sccm}$ gesteigert wurde, zusätzlich zu dem konstanten Ar-Waferheizgasfluss von $Q(\operatorname{ArH})=10 \mathrm{sccm}$. Als Ar-Partialdruck ergeben sich somit Werte von 2,5 - 6,9 mTorr.

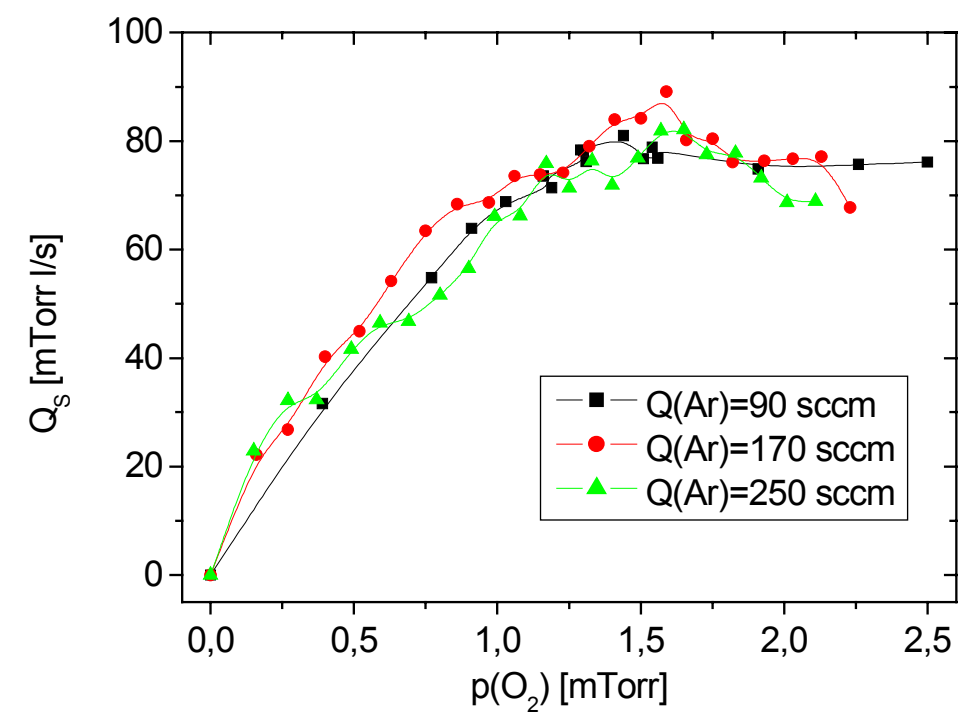

Abb. 3.10: Auftragung des gegetterten Sauerstoffflusses $Q_{S}$ gegen den Sauerstoffpartialdruck für unterschiedliche Ar-Partialdrücke, $s_{p}=440 \mathrm{l} / \mathrm{s}, P=300 \mathrm{~W}, \mathrm{~T}=500^{\circ} \mathrm{C}$

Die in Abb. 3.10 dargestellten Graphen zeigen, dass sich das $Q_{S}-$ Maximum unabhängig vom hier gewählten Ar-Partialdruck bei einem Sauerstoffpartialdruck von $p\left(\mathrm{O}_{2}\right)=1,6$ mTorr einstellt. Aufgrund der leicht unterschiedlichen Saugleistungen für $\operatorname{Ar}$ und $\mathrm{O}_{2}$ entspricht dies einem etwas verändertem reaktiven Sauerstofffluss $\mathrm{Q}\left(\mathrm{O}_{2}\right)$. Bei den gewählten Ar-Flüssen sind im Hinblick auf die Position und den absoluten Wert des Maximums in den Diagrammen keine Unterschiede nachweisbar. Es wird daher gefolgert, dass die Variation des ArPartialdrucks in dem untersuchten Intervall keinen signifikanten Einfluss auf den GetterEffekt nimmt.

\subsection{Diskussion der Herstellungsbedingungen}

Die Analysen mittels generischer Plasmakurven und die Evaluierung des Getter-Effektes über $Q_{S}-p\left(O_{2}\right)$-Diagramme ergeben eine systematische Abhängigkeit des GetterMaximums vom Sputteryield $Y_{\mathbb{I}}$, der über die Sputterleistung geregelt wird, und vom Sauerstoff-Partialdruck, der durch die Pumpleistung und den reaktiven Fluss eingestellt wird. Für 
jeden der gewählten Parametersätze $\left(s_{p}, P, p(A r), T_{S}\right)$ und nicht zu hohe Sputterleistungen kann ein solches Maximum in Abhängigkeit von $\mathrm{p}\left(\mathrm{O}_{2}\right)$ festgestellt werden. Der Einfluss des Argonpartialdrucks $\mathrm{p}(\mathrm{Ar})$ und der Substrattemperatur $\mathrm{T}_{\mathrm{S}}$ während der Abscheidung zeigt in dem gewählten Parameterintervall keinen Einfluss auf die generischen Kurven und muss durch die Analyse der Schichteigenschaften geklärt werden.

Es wird vermutet, dass die Abscheidebedingung, bei der das Maximum des Sauerstoffgetterflusses $Q_{S, \max }$ nachgewiesen wird, dem Optimum der Abscheidebedingungen entspricht und an diesem Arbeitspunkt aufgrund der diskutierten Aspekte zum target poisoning stöchiometrisches $\mathrm{IrO}_{2}$ deponiert wird. Daher muss es das Ziel sein, über die Charakterisierung der Schichteigenschaften einen Zusammenhang mit den generischen Kurven herzustellen. Den Schwerpunkt soll die Analyse der Schichteigenschaften in der Umgebung des Übergangspunktes zur Target-Sauerstoffbelegung (,target poisoning“) bilden. Aus den bereits genannten Gründen sind vor allem Prozessbedingungen mit höheren Depositionsleistungen interessant, bei denen dieser Übergang innerhalb des verfügbaren reaktiven Flussbereiches liegen. Diese Anforderungen führen die Wahl der Depositionsleistung auf Werte von P=300 W bzW. $\mathrm{P}=500 \mathrm{~W}$. Im folgenden werden deswegen die Analysen an den mit diesen Parametern abgeschiedenen Iridiumoxidschichten durchgeführt. 


\section{Physikalische Eigenschaften der $\mathrm{IrO}_{\mathrm{x}}$ Schichten}

Anhand der in Kap. 3.7 angestellten Überlegungen kann bei der reaktiven Deposition der $\mathrm{IrO}_{\mathrm{x}}$ Schichten ein für die Depositionsleistung charakteristisches Maximum in der gegetterten Flussmenge in Abhängigkeit des Sauerstoffpartialdrucks bestimmt werden. In diesem Kapitel sollen die Schichteigenschaften in der Umgebung dieses Punktes analysiert und diskutiert werden.

\subsection{Analyse mittels Depositionsraten}

Die Bestimmung der Schichtdicke geschah mittels Röntgenreflektometrie und Profilometrie. Die gemessenen absoluten Wachstumsraten lagen für Sputterleistungen von $P=300 \mathrm{~W}$ und $P=500 \mathrm{~W}$ im Bereich von $r_{d}=(0,5-2,0) \mathrm{nm} / \mathrm{s}$. Die gemessenen absoluten Werte wurden auf die Ir-Depositionsrate $\left(\mathrm{Q}\left(\mathrm{O}_{2}\right)=0 \mathrm{sccm}\right)$ normiert, um aus den relativen Werten Schlüsse auf die Phasenbildung ziehen zu können. Der Einfluss des Sauerstoffpartialdrucks auf die Wachstumsraten ist in Abb. 4.1 für die genannten Depositionsleistung bei einer gewählten Abscheidetemperatur von $\mathrm{T}_{S}=100^{\circ} \mathrm{C}$ gezeigt.

a)

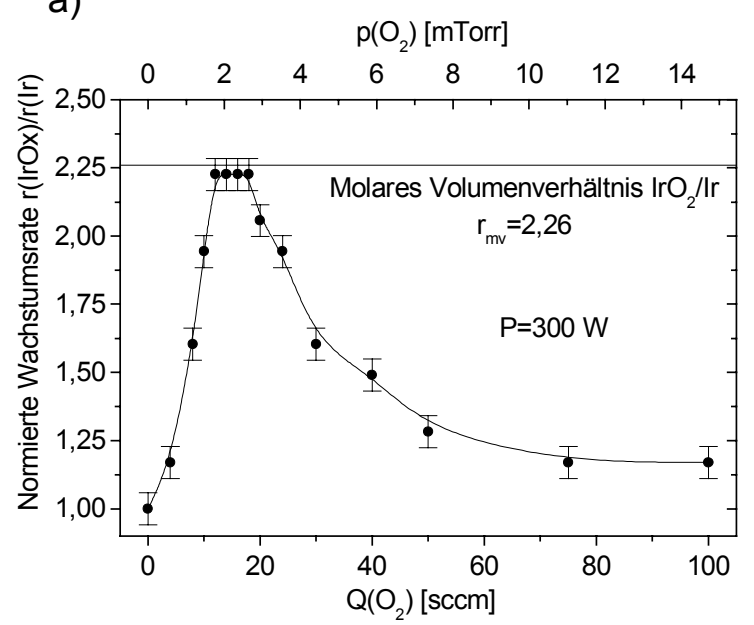

b)

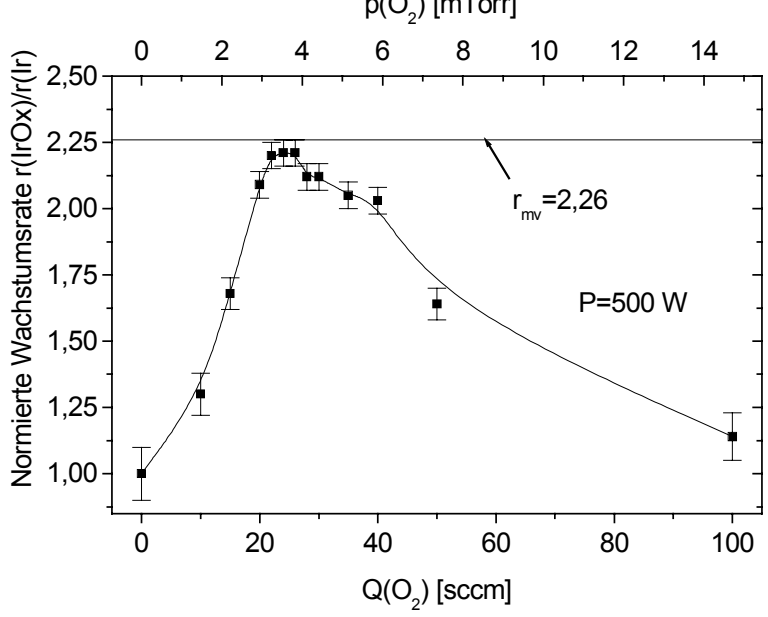

Abb. 4.1: Einfluss des Sauerstoffpartialdrucks auf die Abscheiderate der $\operatorname{IrO}_{x}$ Schichten bei einer Depositionsleistung von a) $300 \mathrm{~W}$ und b) $500 \mathrm{~W}$. Als Substrate wurden oxidierte Si(100) Wafer benutzt, die Schichtdicken wurden mittels Step-Profilometrie ermittelt. Angegeben ist das errechnete relative Verhältnis der molaren Volumina des $\mathrm{IrO}_{2}$ zum Ir als $\mathrm{r}_{\mathrm{mv}}=2,26$.

Mit ansteigendem Sauerstoffpartialdruck wird eine rasche Zunahme der Wachstumsrate beobachtet, die mit einem zunehmenden Einbau von Sauerstoffatomen in die Ir Schicht gedeutet werden kann und zu einer Volumenexpansion führt. Dabei ist die Kondensationsrate der Ir-Atome auf dem Substrat unabhängig vom Sauerstoff-Partialdruck. Für einen höheren Sauerstoffpartialdruck wird eine zunehmende Ausbildung eines Volumen-Anteils der $\mathrm{IrO}_{2}$ Phase 
vermutet, da das molare Volumen des $\mathrm{IrO}_{2}$ um einen Faktor 2,26 größer ist als das des rei-

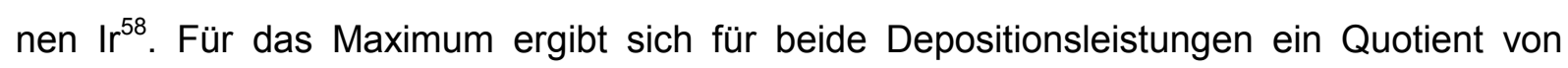
knapp unter dem genannten Wert von 2,26. Aus diesen Daten lässt sich also bereits vermuten, dass sich unter den Depositionsbedingungen am Maximum der Depositionsrate kristallines und nahezu stöchiometrisches $\mathrm{IrO}_{2}$ ausbilden sollte, wenn man uniformes kristallines IrWachstum bei einem Sauerstofffluss von $\mathrm{Q}\left(\mathrm{O}_{2}\right)=0 \mathrm{sccm}$ annimmt. Die Abszissenposition des Maximums der Schichtwachstumsrate ist identisch mit der Lage des Sauerstoff-GetterMaximums der $Q_{S}-p\left(O_{2}\right)$-Diagramme, d.h. der maximale Sauerstoffeinbau fällt mit der jeweils größten Wachstumsgeschwindigkeit der Schichten zusammen.

Für größere Sauerstoffpartialdrücke fällt die Depositionsrate wieder deutlich ab, was hier mit dem reduzierten Ir-Sputteryield $Y_{\text {Ir }}$ im belegten Target-Modus erklärt werden kann [Pow99]. Tatsächlich korreliert die Abnahme der Depositionsrate mit einer systematischen Abnahme des Target-Potentials $U_{b}$ und einer Abnahme des Sauerstoffgetterflusses $Q_{s}$. Die Abnahme der Depositionsrate beträgt bei dem größten untersuchten Sauerstoffpartialdruck etwa einen Faktor zwei im Vergleich zur maximalen Depositionsrate. Vergleichbare Ergebnisse sind für die anderen Depositionstemperaturen gefunden worden, was auf eine von der Substrattemperatur unabhängige Wachstumsgeschwindigkeit schließen lässt.

\subsection{Analyse der chemischen Zusammensetzung mittels Rutherford Rück- streu-Spektrometrie (RBS)}

Mithilfe der Rutherford Rückstreu-Spektrometrie (RBS) soll hier der Zusammenhang zwischen den generischen Kurven und der Zusammensetzung der Schichten hergestellt werden. Dazu wurden sowohl im Depositionsmodus mit ungedrosselter wie auch mit gedrosselter Pumpleistung für verschiedene Depositionstemperaturen die Zusammensetzung der reaktiv abgeschiedenen Schichten in Abhängigkeit des Sauerstoffflusses analysiert und zusätzlich die Kontamination mit dem Sputtergas Argon bestimmt. Für die hier gezeigten Analysen wurde eine Sputterleistung von $\mathrm{P}=300 \mathrm{~W}$ gewählt. Abb 4.2 zeigt RBS-Spektren von $\mathrm{IrO}_{\mathrm{x}}$ Schichten, die bei einer Substrattemperatur von $100^{\circ} \mathrm{C}$ im gedrosselten Pumpmodus abgeschieden wurden. Für die Deposition aller drei Schichten wurde eine konstante Sputterzeit von $\mathrm{t}=170 \mathrm{~s}$ verwendet, was den unterschiedlichen Schichtdicken von $d_{1}=105 \mathrm{~nm}$, $d_{2}=200 \mathrm{~nm}$ und $d_{3}=175 \mathrm{~nm}$ für die drei verwendeten Sauerstoffflüsse von $Q\left(O_{2}\right)=4,14$ bzw. $24 \mathrm{sccm}$ entspricht.

\footnotetext{
${ }^{58}$ Unter Beachtung der Tatsache, dass beim Iridium vier Ir-Atome pro Einheitszelle und beim $\mathrm{IrO}_{2} \mathrm{zwei}$ Moleküle pro Einheitszelle vorhanden sind, lässt sich bei Kenntnis der Größe der kristallinen Einheitszelle (s. Kap. 1.4) das molare Volumen der beiden Materialien berechnen
} 


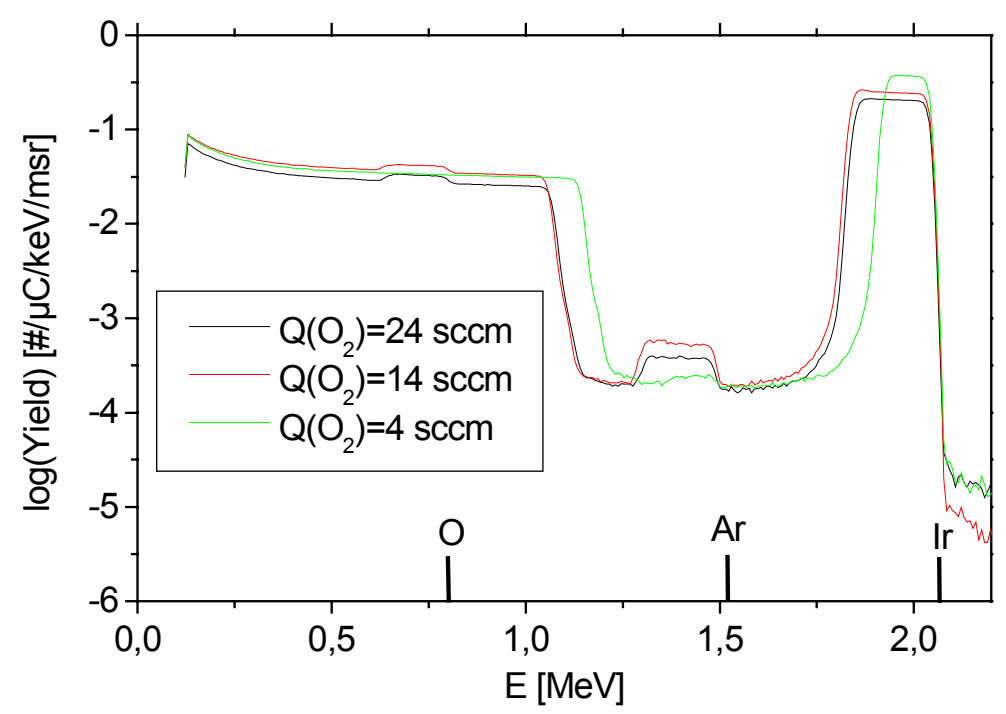

Abb. 4.2: RBS-Spektren von $\mathrm{IrO}_{x}$ Schichten, die mit unterschiedlichem Sauerstoffpartialdruck deponiert wurden. Als Substrat wurden mit HF angeätzte (100)-Si Wafer benutzt; $T_{S}=100^{\circ} \mathrm{C}$, $P=300 \mathrm{~W}$

In Abb. 4.2 sind in den Spektren zusätzlich die Schichtelemente an der hochenergetischen Signalflanke mit eingetragen. Die hochenergetische Flanke entspricht der Energie, die die direkt an der Schichtoberfläche zurückgestreuten He-Ionen besitzen. Durch iterative Anpassung der Parameter an die gemessenen Spektren mittels der Software RUMP erhält man die quantitative Zusammensetzung der Schicht. Die so erhaltenen Daten sind in Abb. 4.3 a) und b) zusammengefasst und direkt mit den in Kap. 3.7 gezeigten Diagrammen der Sauerstoffgetterflüsse $Q_{S}$ verglichen. Dabei sind unterschiedliche Depositionstemperaturen farblich voneinander abgehoben. Deutlich erkennbar aus Abb. 4.3 b) ist die zunächst geringe, dann rasche Zunahme des O/Ir Verhältnisses der Schichten bei der Zugabe von Sauerstoff in das Ar-Plasma. Für einen Sauerstoffpartialdruck, der über dem Sättigungspunkt liegt, ist eine deutlich verlangsamte Zunahme des Sauerstoffgehaltes in den $\mathrm{IrO}_{2+x}$ Schichten sichtbar. Da das $\mathrm{IrO}_{2}$ bereits die höchste im Ir-O-System vorkommende Oxidationsstufe des Iridiums darstellt, ist dieses Sättigungsverhalten in der Aufnahme des reaktiven Sputtergases durch einen deutlich reduzierten Haftungskoeffizient von Sauerstoffatomen an bereits oxidierten Schichtoberflächen zu deuten. Ein solches Sättigungsverhalten ist typisch für reaktiv gesputterte Schichten [Pow99], [Rei95].

Die Verfahrensauflösung für die Bestimmung des Sauerstoffgehalts der Schichten liegt etwa bei 3-5\% [Chu78], anhand Abb. 4.3 a) und b) wird trotz dieser relativen Unsicherheit deutlich, dass unabhängig von der gewählten Systempumpleistung $s_{p}$ der Punkt des maximalen Sauerstoffgetterflusses $Q_{\mathrm{S} \text {, max }}$ identisch mit der Abscheidung von stöchiometrischen $\mathrm{IrO}_{2}$ Schichten ist. Im folgenden werden daher die $\mathrm{IrO}_{2}$ Schichten, die am Punkt des maximalen 
Sauerstoffgetterflusses abgeschieden wurden, auch als stöchiometrische $\mathrm{IrO}_{2}$ Proben bezeichnet. Die Ar-Verunreinigung der Schichten zeigt keine systematische Abhängigkeit vom Sauerstoffpartialdruck während der Deposition und liegt für alle Proben unter $1 \%$.

a)

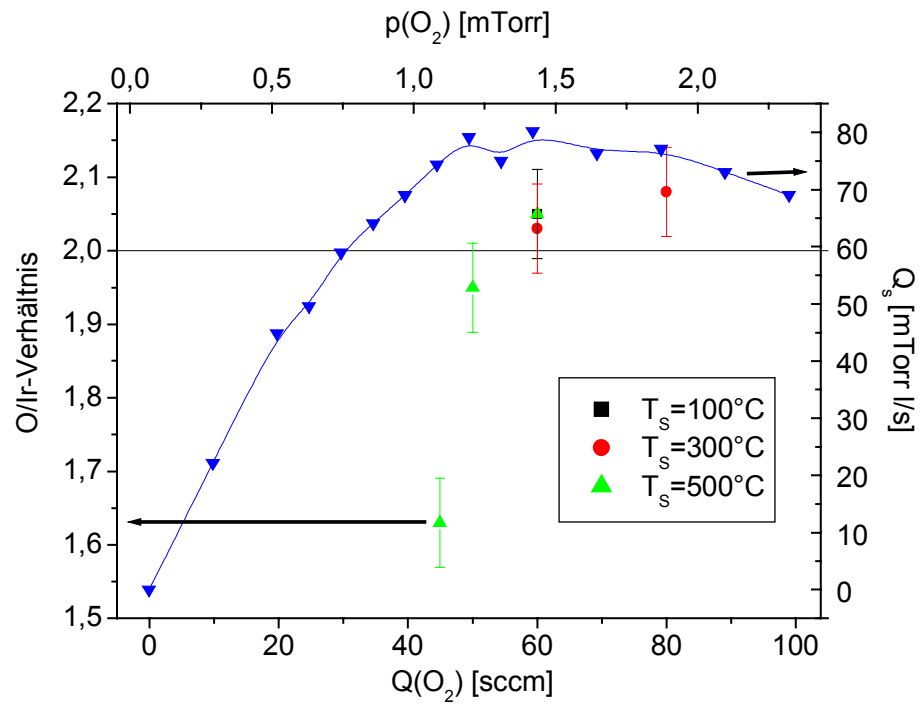

b)

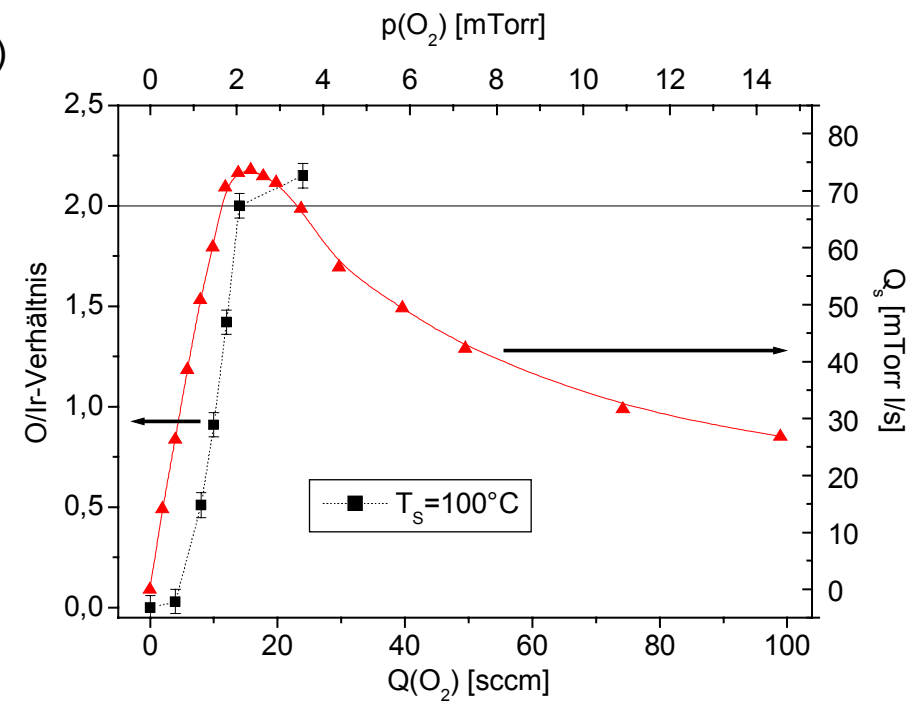

Abb. 4.3: Mittels Simulation der RBS-Spektren bestimmtes O/Ir-Verhältnis für eine Systempumpleistung von a) $s_{p, 1}=440 \mathrm{l} / \mathrm{s}$ und b) $s_{p, 3}=70 \mathrm{l} / \mathrm{s}, \mathrm{P}=300 \mathrm{~W}$. Als Vergleich ist der aus den generischen Kurven errechnete Sauerstoffgetterfluss $Q_{S}$ eingezeichnet, die Linien sind Splines, die zur Führung des Auges dienen. 


\subsection{Charakterisierung der abgeschiedenen Schichten mittels Röntgendiffrak- tometrie (XRD)}

Mit der Röntgendiffraktometrie soll die Phasenbildung analysiert und eine mikrostrukturelle Charakterisierung der reaktiv gesputterten Schichten in Abhängigkeit der untersuchten Depositionsparameter Sauerstoffpartialdruck, Substrattemperatur und Depositionsleistung durchgeführt werden. Dabei sollen die optimalen Depositionsparameter für die $\mathrm{IrO}_{2}$ Phasenbildung gefunden und mit den Ergebnissen der Berechnungen aus den generischen Plasmakurven verglichen werden. Überdies ist zu klären, ob eine Nichtgleichgewichtsphasenbildung neben den zwei bekannten Solidus-Phasen $\mathrm{Ir}$ und $\mathrm{IrO}_{2}$ beobachtbar ist. Die thermische Stabilität der erhaltenen Phasen wird in Kap. 5 unterSucht. Neben Untersuchungen zur Phasenformation werden hier mittels der Röntgendiffraktometrie ebenfalls Aussagen über die Textur und über auftretende Veränderungen in der Gitterkonstante gemacht, die Informationen über Spannungen und intrinsische Defekte in der Probe enthalten. Diese Aspekte sind wichtige Gesichtspunkte zur Funktionalität und Verwendbarkeit der Schichten als Diffusionsbarriere. Detaillierte Analysen zu Korngrößen werden in Kap. 4.6 zur Tunnelmikroskdpie angestellt.

In Abb. 4.4 sind Röntgendiffraktometriespektren der reaktiv gesputterten Schichten gezeigt, die bei ein $r$ Substrattemperatur von $T_{S}=100^{\circ} \mathrm{C}$ im gedrosselten Pumpmodus $\left(s_{p, 3}=70 \mathrm{l} / \mathrm{s}\right)$ hergestellt wurden. Daher entspricht der in den Diffraktometrie-Spektren angegebene Sauerstofffluss von 0 bis $100 \mathrm{sccm}$ einem $\mathrm{O}_{2}$-Partialdruck von 0 bis 15 mTorr. Als Substratmaterial wurden Si(100) Wafer mit einer $675 \mathrm{~nm}$ dicken thermischen Oxidschicht benutzt, um Substrateffekte ausschließen zu können. Unabhängig von der gewählten Depositionsleistung (Abb. 4.4 a: $P=300 \mathrm{~W}$ und b: $\mathrm{P}=500 \mathrm{~W}$ ) lassen sich verschiedene Regionen der reaktiven Abscheidyng der Phasenbildung abgrenzen. Für die ohne reaktives Gas abgeschiedene Schichten erkennt man, dass sich jeweils eine stark (111)-texturierte Ir Schicht gebildet hat. Diese Vorzugsorientierung ist nicht ungewöhnlich für die Abscheidung von kubisch flächenzentrierten Materialien, da die (111)-Ebenen in dieser Struktur die dichtest gepackten Ebenen darstellen [Hec81], [Leg94]. Mittels Scherrer-Auswertung errechnet sich eine mittlere IrKorngröße von etwa $d_{c o h}=10 \mathrm{~nm}$. Durch Hinzufügen einer geringen Menge reaktiven Sauerstoffgases, was hier für die weitere Diskussion als Region 1 bezeichnet wird, ändert sich die Mikrostruktur in eine schwachkristallin/amorphe Mischung aus Ir und $\mathrm{IrO}_{2}$. Da die kubische IrPhase mit der hohen Dichte von $\rho=22,55 \mathrm{~g} / \mathrm{cm}^{3}$ nur sehr geringe Mengen Sauerstoff substitutionell bzw. interstitiell lösen kann [Gme78], ist das Verschwinden der Kristallinität mit zunehmendem Sauerstoffeinbau verständlich, da mit dem Übersättigen eine große Anzahl an Defekten in die Schicht eingebaut wird. Es ist anzunehmen, dass der Exzess-Sauerstoff jedoch auch in den Korngrenzen der Ir Schicht eingebaut wird. Bei weiterer Erhöhung des $\mathrm{O}_{2}-$ Drucks, entsteht in einem relativ engen Partialdruckbereich, der als Region 2 bezeichnet 
wird, eine reinphasige kristalline $\mathrm{IrO}_{2}$ Schicht mit orientierungsabhängigen Korngrößen von etwa $d=5 \mathrm{~nm}$ für (200)-orientierte Körner und $d=15 \mathrm{~nm}$ für (110) orientierte Körner. Kennzeichnend für die bei $100^{\circ} \mathrm{C}$ abgeschiedenen $\mathrm{IrO}_{2}$ Schichten ist eine deutlich ausgeprägte (200)-Textur. Für untexturierte Proben (Pulverproben) wird ein Intensitätsverhältnis von $T=I(110) /(200)=4$ angegeben [JCP95].
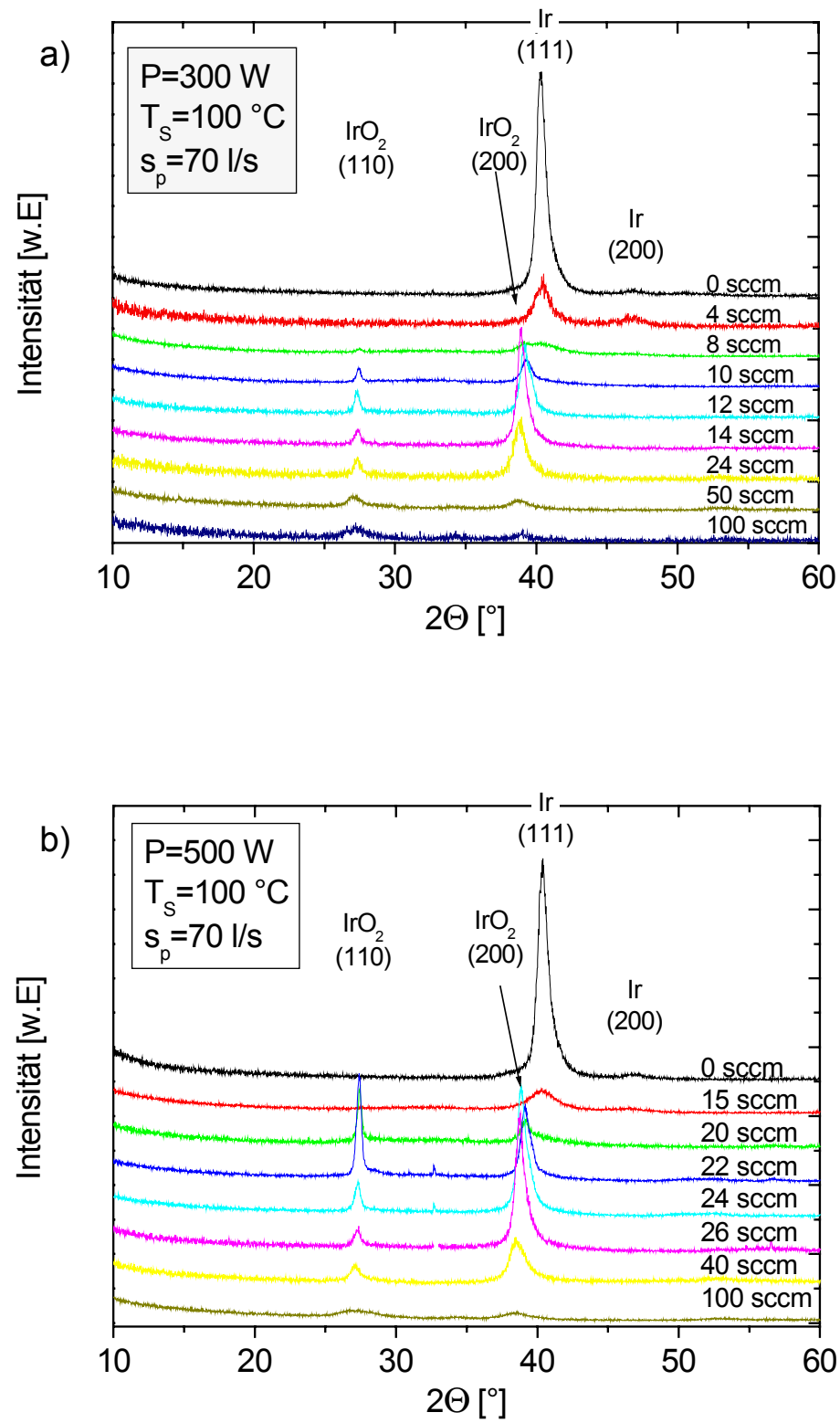

Abb. 4.4: Röntgendiffraktometriespektren der bei a) $300 \mathrm{~W}$ und b) $500 \mathrm{~W}$ gesputterten $\mathrm{IrO}_{\mathrm{x}}$ Schichten. Als Substrate wurden thermisch oxidierte $\mathrm{Si}(100)$ Wafer benutzt. Die für die Deposition verwendeten Sauerstoffgasflüsse sind an den Spektren angegeben, diese entsprechen bei der gewählten Saugleistung $\mathrm{s}_{\mathrm{p}}=70 \mathrm{l} / \mathrm{s}$ einem Partialdruck von $\mathrm{p}\left(\mathrm{O}_{2}\right)=0 . .15 \mathrm{mTorr}$; $\mathrm{T}_{S}=100^{\circ} \mathrm{C}$, Schichtdicke $\mathrm{d}\left(\operatorname{IrO}{ }_{\mathrm{x}}\right)=100 \mathrm{~nm}$ 
Bei weiterer Steigerung des Sauerstoffpartialdrucks (Region 3) verschlechtert sich die Kristallinität der Schichten graduell, was der Sauerstoffüberstöchiometrie der Schichten zugeschrieben wird, die ein ungestörtes Wachstum von $\mathrm{IrO}_{2}$-Körnern verhindert. Zudem gibt Gmelin [Gme78] auch für die $\mathrm{IrO}_{2}$-Phase nur eine sehr geringe Exzess-Sauerstofflöslichkeit an. Bei den hier untersuchten Sauerstoffpartialdrücken von 0..15 mTorr können keine weiteren Phasen identifiziert werden. Weiterhin kann gezeigt werden, dass sich die Region 2, in der sich kristalline, reinphasige $\mathrm{IrO}_{2}$ Schichten bilden, zu höheren Sauerstoff-Partialdrücken verschiebt. Dies steht in guter Übereinstimmung mit den Analysen aus Kap.3.7.3.

Um den Phasenbildungsprozess in der Nähe des Übergangspunktes (Region 2) genauer zu analysieren, wurde die reaktive Deposition im ungedrosselten Pumpmodus $\left(s_{p, 1}=4401 / s\right)$ bei verschiedenen Substrattemperaturen untersucht. Anhand der dazu aufgenommenen, hier aber nicht gezeigten Spektren und der Texturanalysen ist nachweisbar, dass bei einer Substrattemperatur von $100^{\circ} \mathrm{C}$ und $300^{\circ} \mathrm{C}$ die $\mathrm{IrO}_{2}$ Phase generell nur die Existenz des (110)und des (200)-Reflexes aufzeigt. Dabei ist aber ausnahmslos eine deutlich ausgeprägte (200)-Textur zu beobachten. Weiterhin ist bemerkenswert, dass der ebenfalls niedrig indizierte, für die Referenzprobe intensitätsstarke (101)-Reflex in nahezu keinem gemessenen Spektrum sichtbar ist, d.h. dass sich die (101)-Ebenen nicht in der Schichtebene liegen.

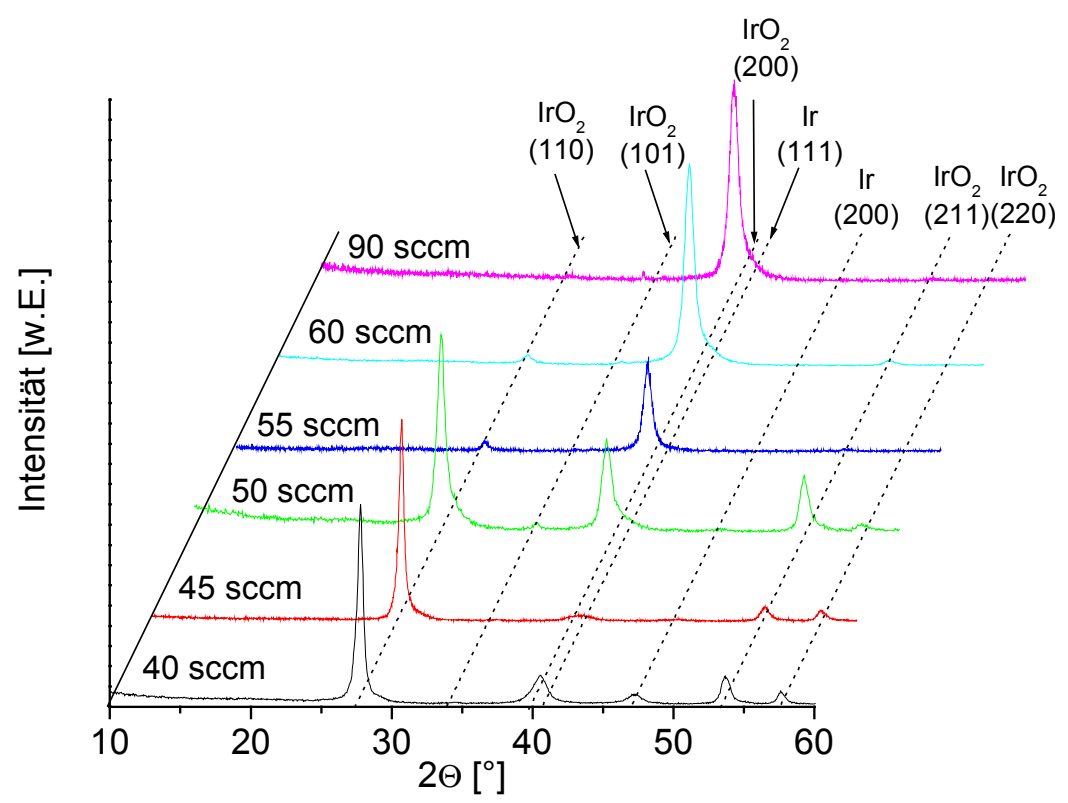

Abb. 4.5: Weitwinkelbeugungs-Spektren bei reaktiver $1 \mathrm{IO}_{\mathrm{x}}$ Abscheidung in Region $2 . \mathrm{T}_{\mathrm{S}}=500^{\circ} \mathrm{C}$, $\mathrm{P}=300 \mathrm{~W}, \mathrm{~s}_{\mathrm{p}, 1}=440 \mathrm{l} / \mathrm{s}$. Als Substrate wurden thermisch oxidierte Si(100) Wafer benutzt, die Schichtdicke beträgt $d=100 \mathrm{~nm}$. Die für die Schichtdeposition verwendeten Sauerstoffgasflüsse sind jeweils angegeben. 
Die in Abb. 4.5 angegebenen Sauerstoffflüsse von 40 bis 90 entsprechen bei der ungedrosselten Pumpleistung einem $\mathrm{O}_{2}$-Partialdruck von 1,0 bis etwa 2,2 mTorr. Bei den $1 \mathrm{rO}_{\mathrm{x}}$ Schichten, die bei einer Substrattemperatur von $500^{\circ} \mathrm{C}$ abgeschieden wurden und in Abb. 4.5 gezeigt sind, lassen sich bei Flüssen von 40 und $45 \mathrm{sccm}$ neben der $\mathrm{IrO}_{2}$ Phase noch Volumenanteile von sehr feinkristallinem Ir nachweisen, d.h. die Phasenbildung des Ir geht bei dieser Temperatur mit zunehmendem $\mathrm{O}_{2}$-Partialdruck direkt in die Phasenbildung des $1 \mathrm{rO}_{2}$ über, ohne dass sich eine amorphe Mikrostruktur nachweisen lässt. In einem sehr engen Sauerstoffpartialdruckbereich, der Flüssen zwischen 40 und $55 \mathrm{sccm}$ entspricht, sind aber im Gegensatz zu niedrigeren Substrattemperaturen auch höher indizierte Ebenen-Reflexe (z.B. (211) und (220)) des $\mathrm{IrO}_{2}$ Spektrums mit unterschiedlichen Intensitäten sichtbar. Da diese Reflexe bei höheren Sauerstoffflüssen jedoch nicht zu finden sind, scheint die Nukleation und das Wachstum anders als (200)-orientierter Körner bei höheren Partialdrücken wieder unterdrückt zu werden.

Eine Abschätzung der Korngrößen aus den Halbwertsbreiten ergibt für den schmalen Flussbereich, in dem die größte Anzahl an Reflexen des $\mathrm{IrO}_{2}$ Spektrums auftreten, Größen von etwa 10-25 nm. Die quantitative Auswertung der Schichttexturen in Abb. 4.6 zeigt, dass sich bei einer Substrattemperatur von $500^{\circ} \mathrm{C}$ ein schlagartiger Übergang von einer (110)- in eine (200)-Textur mit zunehmendem Sauerstoffpartialdruck ausbildet.

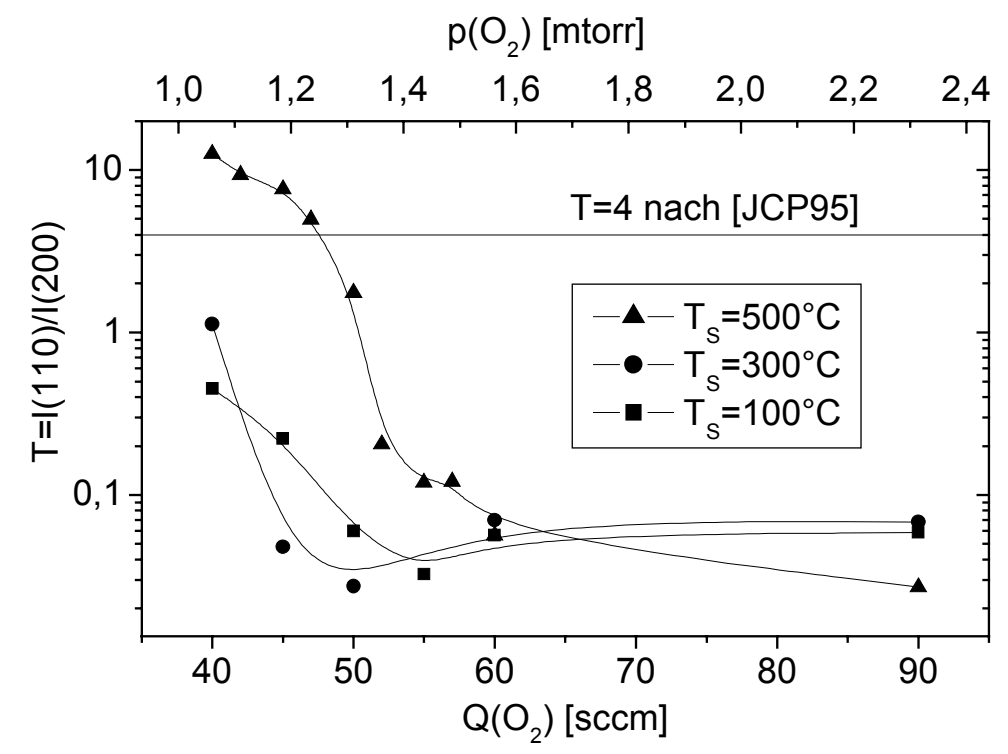

Abb. 4.6: Texturanalysen als Funktion des Sauerstoffpartialdrucks $p\left(O_{2}\right)$ für verschiedene Depositionstemperaturen $T_{S}$ in der Umgebung des Punktes des maximalen Sauerstoff-GetterFlusses $Q_{S}$, max. Als Substrat wurden thermisch oxidierte Si-Wafer benutzt, die Schichtdicke beträgt $\mathrm{d}(\operatorname{IrO} \mathrm{x})=100 \mathrm{~nm}$. Die eingezeichneten Linien dienen zur Führung des Auges. Als Referenz ist das Texturverhältnis einer Pulverprobe eingezeichnet ( $T=4)$ [JCP95]. 
Die relative Änderung des oben definierten Texturverhältnisses T über drei Größenordnungen zeigt deutlich die Empfindlichkeit der Textur in bezug auf den gewählten SauerstoffPartialdruck. Als Referenz ist das Intensitätsverhältnis einer untexturierten Pulverprobe $T(110) / T(200)=4$ in Abb. 4.6 mit eingezeichnet. Für die anderen untersuchten Substrattemperaturen wurden unabhängig vom Sauerstoffpartialdruck immer (200)-texturierte $\mathrm{IrO}_{2}$ Schichten gefunden. Der Grad der vorgefundenen (200)-Textur wird zunächst mit zunehmendem Sauerstoffpartialdruck deutlicher und bleibt für sehr hohe Partialdrücke nahezu konstant. Die Entstehung von Texturen in gesputterten Schichten kann durch mehrere Ursachen hervorgerufen werden, wie beispielsweise durch epitaktisches Wachstum, lonenbeschuss während der Deposition [Yu86], [Har78], oder durch Oberflächenrekonstruktion [Ber76], [Hud77]. Die Rekonstruktion der Kristalloberflächen dient zur Energieminimierung der freien Oberflächen und ist für einige Oxide wie $\mathrm{TiO}_{2}$ [Mur94], $\mathrm{SnO}_{2}$ [Cox89], oder $\mathrm{Al}_{2} \mathrm{O}_{3}$ [Ren94], untersucht worden. Dabei stellen die Stöchiometrie und die OberflächenLeerstellenkonzentrationen wichtige Parameter dar, die den Rekonstruktionsprozess beeinflussen. Im tetragonalen Gitter des $\operatorname{IrO}_{2}$ (und des $\mathrm{TiO}_{2}$ ) stellen die (110)-Ebenen die dichtest gepackten Ebenen dar, diese Schichtvorzugsorientierung kann hier aber ausschließlich bei hohen Substrattemperaturen und nicht zu hohem Sauerstoffpartialdruck nachgewiesen werden. Die anderen vorgestellten Spektren zeigen vielmehr eine Wachstumsorientierung, bei der bevorzugt die (200)-Ebenen senkrecht zur Schichtnormalen aufwachsen. Die geschilderte Abhängigkeit der Textur vom Sauerstoffpartialdruck lässt den Schluss zu, dass der energetische Beschuss von Sauerstoffionen bzw. am Target neutralisierten und reflektierten Sauerstoffatomen ein wichtiger Parameter ist, der die Textur der $\mathrm{IrO}_{\mathrm{x}}$ Schichten beeinflussen kann. Daher wird dieser Beschuss in die aufwachsenden Schichten im folgenden genauer diskutiert.

Untersuchungen zur Position der Lagen der $\mathrm{IrO}_{2}$ Reflexe zeigen eine relative Verschiebung zur jeweiligen Referenzlage zu kleineren Beugungswinkeln. Da bei den meisten Proben der (200)-Peak besonders stark ausgeprägt ist, wird für die systematische Analyse der Peaklage in Abhängigkeit des Sauerstoffpartialdrucks dieser Reflex zur Auswertung herangezogen. Anhand der Braggschen Gleichung [Cul78]

$\lambda=2 d_{h k l} \cdot \sin \theta$

mit $\lambda$ als Wellenlänge der benutzten $\mathrm{Cu}-\mathrm{K}_{\alpha}$ Röntgenstrahlung folgt aus einer Verschiebung zu kleineren Beugungswinkeln $\theta$ eine Aufweitung der (200)-Ebenenabstände. Für ein tetragonales Gitter errechnet sich mit Gleichung (4.1) die Gitterkonstante $a$ aus dem Netzebenenabstand $d_{h k l}$ mit den Millerschen Indizes $\mathrm{h}=2, \mathrm{k}=0, \mathrm{l}=0 \mathrm{zu}$ 


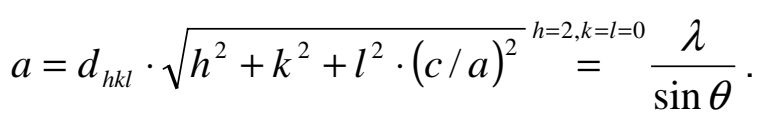

Die so errechnete Gitterkonstante wurde in Abhängigkeit vom Sauerstoffpartialdruck für die untersuchten Substrattemperaturen in Abb. 4.7 und Abb. 4.8 aufgetragen und verglichen. Als untere Begrenzung für den Sauerstofffluss wurde $Q\left(\mathrm{O}_{2}\right)=40 \mathrm{sccm}$ gewählt, da nur oberhalb dieses Flusses einheitlich für alle Temperaturen die Bildung der $\mathrm{IrO}_{2}$ Phase beobachtbar ist. Anhand Abb. 4.7 ist nachweisbar, dass für alle untersuchten Substrattemperaturen mit zunehmendem Sauerstoffpartialdruck eine anwachsende Dilatation der Gitterkonstanten $a$ in der Wachstumsrichtung der Schicht stattfindet und diese Dilatation in eine Sättigung für größere Partialdrücke übergeht. Bei der höchsten gewählten Substrattemperatur von $500^{\circ} \mathrm{C}$ ist es für den kleinsten Sauerstoff-Partialdruck, bei dem die $\mathrm{IrO}_{2}$ Phasenbildung auftritt, möglich, eine $\mathrm{IrO}_{\mathrm{x}}$ Schicht mit einem relaxierten Gitterparameter abzuscheiden. Allerdings ist diese Schicht nicht einphasig, sondern besteht zusätzlich aus einigen Nanometer großen IrKörnern. Der Grad der Gitterverzerrung in der Sättigung ist umso größer, je geringer die Abscheidetemperatur gewählt wird: bei $500^{\circ} \mathrm{C}$ beobachtet man eine Gitterdilatation von etwa $2 \%$, für die Abscheidung bei $100^{\circ} \mathrm{C}$ sogar eine relative Verzerrung von über $3 \%$. Diese hohen Gitterdehnungen deuten auf eine mit dem Sauerstoffpartialdruck zunehmende Defektdichte hin, die durch den zunehmenden Sauerstoffionen- bzw. Neutralteilchenbeschuss während des Depositionsprozesses verursacht wird.

Es ist aus Abb. 4.7 weiterhin zu erkennen, dass alle stöchiometrisch abgeschiedenen $\mathrm{IrO}_{2}$ Schichten $\left(\mathrm{Q}\left(\mathrm{O}_{2}\right)=60 \mathrm{sccm}\right)$ bei den gewählten Depositionsbedingungen eine deutliche Gitterverzerrung aufweisen. Die in Abb. 4.8 dargestellten Daten sind an Schichten gemessen worden, die mit $\mathrm{P}=300 \mathrm{~W}$ bzw. $\mathrm{P}=500 \mathrm{~W}$ bei einer Substrattemperatur von $100^{\circ} \mathrm{C}$ deponiert wurden, und deren Diffraktogramme in Abb. 4.4 präsentiert sind. Anhand dieser Daten ist zu erkennen, dass die beobachtete Gitterdilatation auch eine Abhängigkeit von der Depositionsleistung aufweist. Mit einer höheren Sputterleistung von $\mathrm{P}=500 \mathrm{~W}$ abgeschiedene Schichten zeigen eine noch höhere relative Gitterdilatation von bis zu 4\%. Diese bei sehr hohen Sauerstoffpartialdrücken abgeschiedenen Schichten zeigen in den Röntgenbeugungsspektren eine extrem schwache Kristallinität, d.h. breite und intensitätsschwache Beugungsreflexe. Stöchiometrische Schichten sind für die in Abb. 4.8 gezeigten Bedingungen bei einem Sauerstofffluss von $14 \mathrm{sccm}(\mathrm{P}=300 \mathrm{~W})$ und bei $24 \mathrm{sccm}(\mathrm{P}=500 \mathrm{~W})$ gemessen worden. 


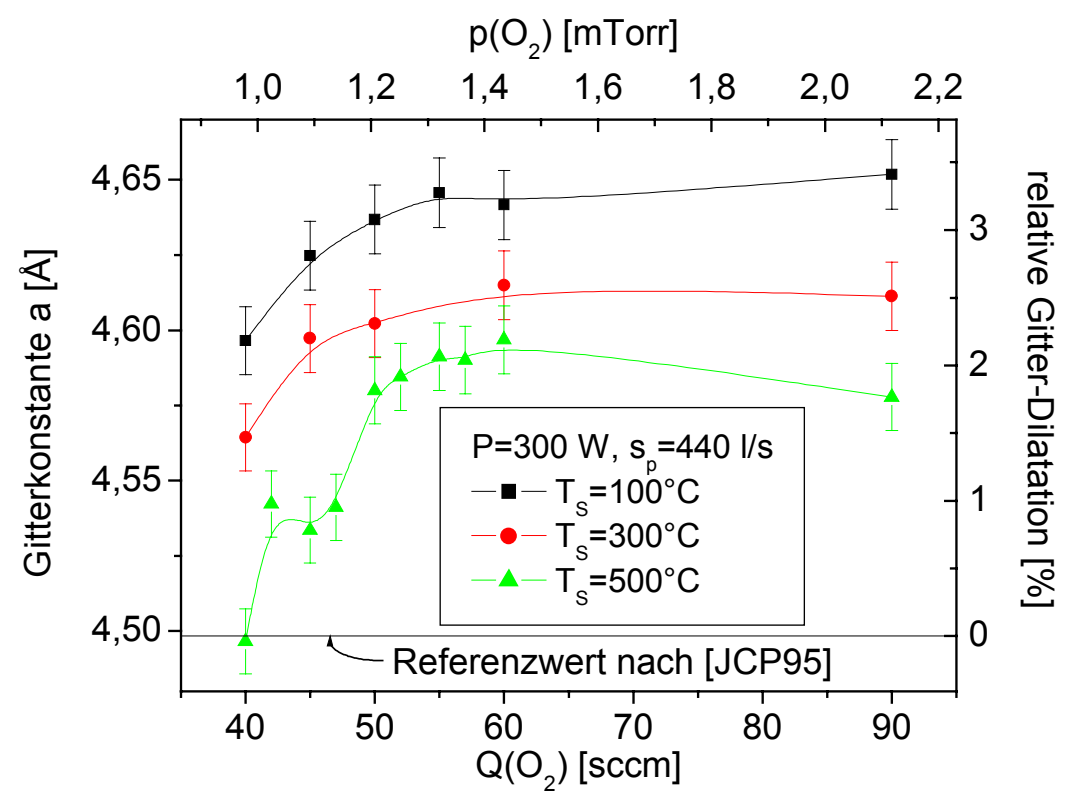

Abb. 4.7: Mittels XRD aus dem (200)-Reflex bestimmter Gitterparameter und relative Dilatation in Abhängigkeit vom $\mathrm{O}_{2}$-Partialdruck und der Substrattemperatur $\mathrm{T}_{\mathrm{S}}$. Der Wert für Pulverproben $(a=4,4983 \AA$ [JCP95]) ist als Referenzlinie eingezeichnet. Die Sputterleistung betrug $\mathrm{P}=300 \mathrm{~W}$, der Argonpartialdruck $\mathrm{p}(\mathrm{Ar})=2,5 \mathrm{mTorr}$. Die eingezeichneten Linien dienen zur Führung des Auges.

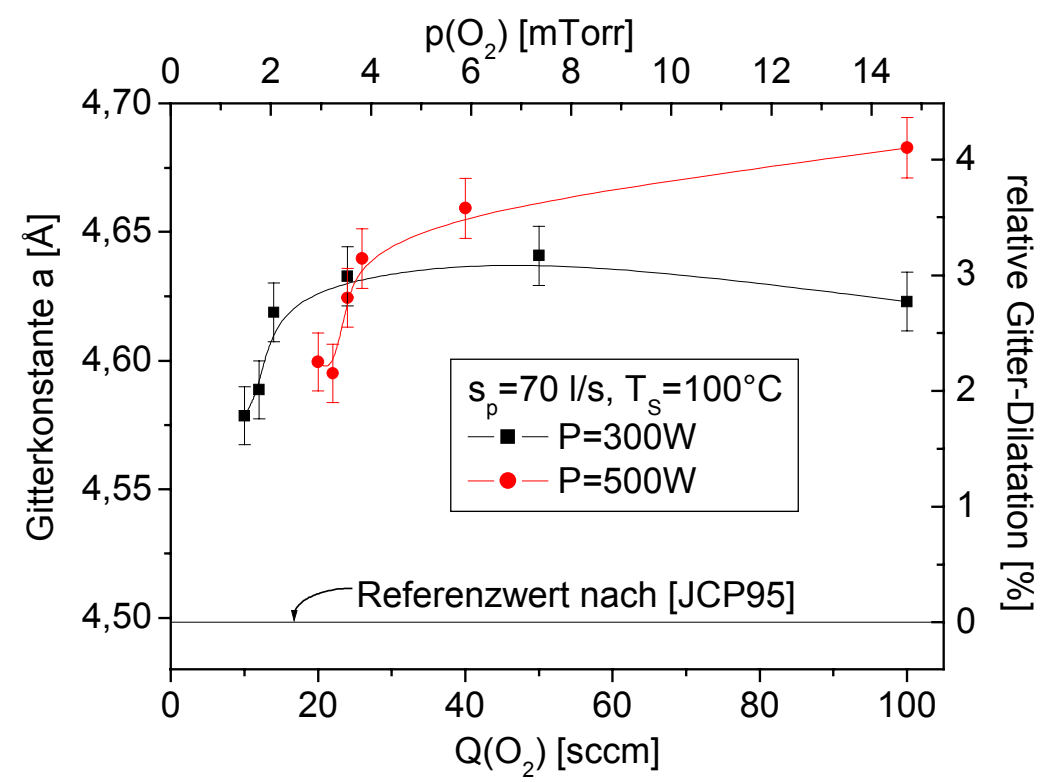

Abb. 4.8: Mittels XRD aus dem (200)-Reflex bestimmter Gitterparameter und relative Dilatation in Abhängigkeit vom $\mathrm{O}_{2}$-Partialdruck. Die Sputterleistung betrug $300 \mathrm{~W}$ und $500 \mathrm{~W}$, die Substrattemperatur $\mathrm{T}_{S}=100^{\circ} \mathrm{C}$ und der Argonpartialdruck $\mathrm{p}(\mathrm{Ar})=2,5 \mathrm{mTorr}$. Der Wert für Pulverproben $(a=4,4983 \AA$ [JCP95]) ist als Referenzlinie eingezeichnet. Die eingezeichneten Linien dienen zur Führung des Auges. 
Zur Beschreibung dieses Effektes sollen Arbeiten von Windischmann herangezogen werden [Win87], [Win91], [Win92]. Windischmann hat, basierend auf der Theorie von linearen Stoßkaskaden, eine quantitative Beschreibung des „atomic shot-peening“-Prozesses ${ }^{59}$ entwickelt. Bei diesem Prozess wird die Energie der Argon- und Sauerstoff-Ionen, die als Neutralteilchen elastisch an den Target-Atomen reflektiert werden, als kinetischer Beschuss in die aufwachsende Schicht transferiert. Dabei ist die transferierte Energie T abhängig von der Projektilenergie $E$, der Projektilmasse $M_{1}$, der Masse des Schichtatoms $M_{2}$ und dem Rückstreuwinkel $\varphi[$ Beh91]

$$
T=\frac{4 M_{1} M_{2}}{\left(M_{1}+M_{2}\right)^{2}} E \cdot \cos ^{2} \varphi
$$

Es wird weiterhin angenommen, dass die Schichtatome unter diesem energetischen Beschuss während des Sputterprozesses aus oberflächennahen Bereichen durch Primär- und Sekundärstöße aus ihren energetisch günstigen Kristallgitterpositionen in Zwischengitterpositionen und in Korngrenzen gebracht werden, wenn der Energieübertrag der Gasatome die Bindungsenergie der Schichtatome, die sogenannte Wigner-Energie, übersteigt [Tho85], [Hof82]. Diese Wigner-Energie liegt für Metalle etwa zwischen 10 und 50 eV [Hor95]. Dieser von Thornton und Hoffman an Sputterschichten untersuchte Prozess hat eine lokale Gitterdehnung als Konsequenz. Gerade bei der Sputterdeposition von Elementen mit großer atomarer Masse $\mathrm{M}_{\mathrm{t}}$ bzw. bei einem hohen Verhältnis von Targetatom-Masse zu Sputtergasmasse und geringem Argonpartialdruck sind besonders hohe intrinsische Spannungen gemessen worden [Cho95], [Tho81]. Dies wird dadurch erklärt, dass die freie Weglänge der Projektile vergleichbar mit dem Target-Substrat-Abstand ist und es daher nicht zu einer Thermalisierung der Projektile kommt. Die Abhängigkeit des shot-peening-Effektes von der Target-Atom-Masse $M_{t}$ ist durch die mit $M_{t}$ gemäß

$\frac{E^{r e f l}}{E^{i n c}}=\frac{\left(M_{t}-M_{i o n}\right)^{2}}{\left(M_{t}+M_{i o n}\right)^{2}}$

steigenden Rückstoß-Energien $E^{\text {refl }}$ der im Kathodenschild auf die Energie $E^{\text {inc }}$ beschleunigten und elastisch reflektierten Gas-lonen mit der Masse $M_{i o n}$ von Thornton und Hoffman gedeutet worden [Tho84]. Das besonders hohe Verhältnis von $M_{I r} / M_{O}=12,0 \mathrm{bzw}$. $M_{I r} / M_{A r}=4,8$, der zur Deposition benutzte niedrige Argonpartialdruck von $\mathrm{p}(\mathrm{Ar})<3,5 \mathrm{mTorr}$ und der geringe Target-Substrat-Abstand von $\mathrm{d}=50 \mathrm{~mm}$ deuten auf die Ursache der diskutierten Gitterdilatationen durch diesen Mechanismus hin. Darüber hinaus muss in einem

\footnotetext{
${ }^{59}$ Atomic shot-peening (engl.) = atomares Strahl-Hämmern
} 
Sauerstoffplasma aufgrund der Elektronenaffinität auch das energetische Anionenbombardement der Schicht für höhere $\mathrm{O}_{2}$-Partialdrücke berücksichtigt werden [Ros89].

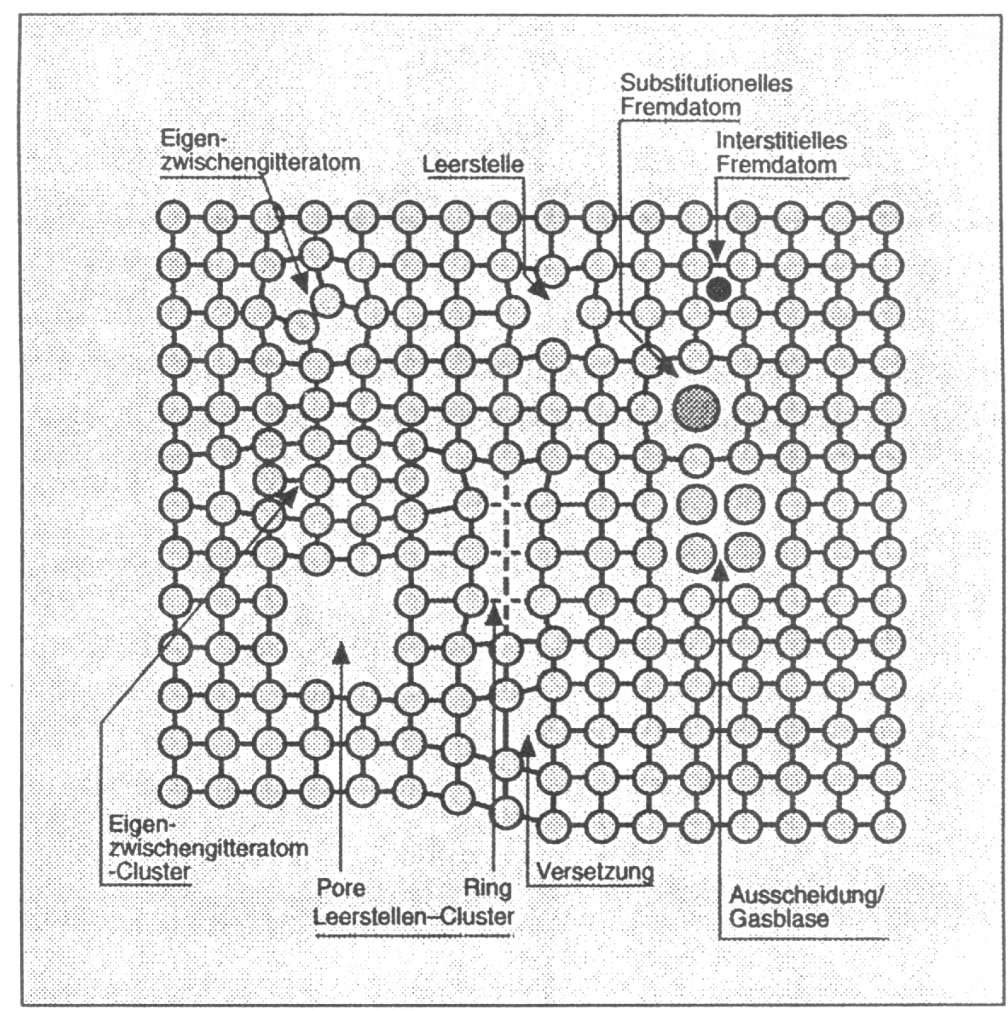

Abb. 4.9: Zweidimensionale, schematische Darstellung von den im Text diskutierten Defekten, die unter energetischem Teilchenbeschuss in einem Kristallgitter entstehen können, aus [Tri94]

Für das Bombardement eines Festkörpers mit energiereichen Primärteilchen wird von Haasen [Haa94] eine Vielzahl möglicher entstehender Defekte diskutiert. Dazu gehören neben den Leerstellen und Zwischengitteratomen auch Verlagerungskaskaden, Crowdionen und verdünnte Zonen. Aufgrund der diskutierten Energien der Gas-lonen im Bereich von einigen 10 bis $100 \mathrm{eV}$ [Ros89] ist die Entstehung der in Abb. 4.9]schematisch dargestellten Defekte, die mit einer Gitterdilatation oder Kompression einhergehen, in der deponierten Schicht erklärbar. Die Schädigungstiefe ist von der Energie und der Masse der eintreffenden lonen abhängig [Beh91], für die hier diskutierten Effekte, bei denen nicht zu hohe lonenmassen $(O$, $\mathrm{O}_{2}$ und $\mathrm{Ar}$ ) und Projektilenergien deutlich unter $1 \mathrm{keV}$ liegen, spricht man vom sogenannten „Single-Knockon-Regime“. In diesem Regime ist die Kaskadendichte gering und es sind nur wenige Atome an den oberflächennahen Stoß-Prozessen beteiligt.

Die für alle Depositionstemperaturen beobachteten, allerdings zu höheren Abscheidetemperaturen deutlich reduzierten Dehnungen lassen vermuten, dass aufgrund der bei niedrigen Temperaturen reduzierten Oberflächenkinetik ein Einfrieren der erzeugten Defekte stattfin- 
det. Bei höheren Abscheidetemperaturen sind der Deposition thermisch aktivierte Prozesse überlagert, die zu einer partiellen Oberflächen- oder Volumen-Relaxation führen.

Die Tatsache, dass sich mit einer Sputterleistung von $\mathrm{P}=500 \mathrm{~W}$ höhere Gitterdilatationen im Vergleich zu einer Leistung von $\mathrm{P}=300 \mathrm{~W}$ am $\mathrm{IrO}_{2}$ nachweisen lassen, lässt sich mit dem Modell von Windischmann zwanglos dadurch erklären, dass die geladenen Sauerstoff-Ionen im Kathodenschild aufgrund der höheren Feldstärke eine höhere kinetische Energie erlangen und nach der elastischen Reflektion am Target eine dementsprechend größere Energie in die Schicht transferieren. Analoges gilt für die Sauerstoff-Anionen, die direkt auf das Substrat beschleunigt werden.

\subsection{Schichtcharakterisierung mittels Röntgenreflektometrie (XRR)}

Mit der Röntgenreflektometrie wurden neben den Schichtdicken die Dichten und die Rauigkeiten der abgeschiedenen Schichten bestimmt. Dazu wurde, wie in Kap. 2.1.1 im Detail beschrieben, an die gemessenen Reflektometriedaten eine Simulation mit einem Chi-Quadrat Algorithmus angepasst. Ein typisches Reflektometriespektrum einer bei $500^{\circ} \mathrm{C}$ gesputterten $\mathrm{IrO}_{2}$ Probe auf einem thermisch oxidierten Si-Wafer ist in Abb. 4.10 gezeigt.

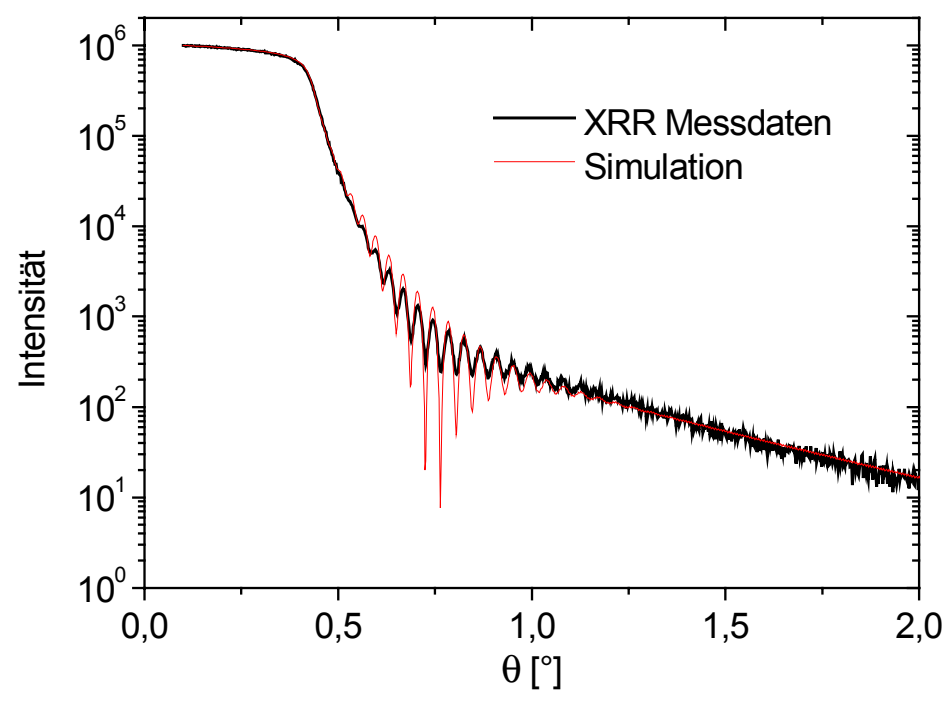

Abb. 4.10: XRR Intensitätsverlauf (schwarz) einer bei $500^{\circ} \mathrm{C}$ gesputterten $\mathrm{IrO}_{2}$ Probe und zugehörige Simulation (rot), $d\left(\mathrm{IrO}_{2}\right)=91,0 \mathrm{~nm}$ auf $\mathrm{SiO}_{2} / \mathrm{Si}$-Substrat, $d\left(\mathrm{SiO}_{2}\right)=625 \mathrm{~nm}$

Die in Abb. 4.10 dargestellte Messung zeigt die Reflektometriemessung und die zugehörige Simulation der bei einer Substrattemperatur von $500^{\circ} \mathrm{C}$ gesputterten stöchiometrischen $\mathrm{IrO}_{2}$ Schicht. Die Simulation ergibt eine Schichtdicke von $d=91,0 \mathrm{~nm}$, eine mittlere quadratische Rauigkeit von $\delta=2,0 \mathrm{~nm}$ und eine fast den Massivproben entsprechende Dichte von 
$\rho=11,3 \mathrm{~g} / \mathrm{cm}^{3} . \mathrm{Im}$ Vergleich dazu ergibt sich für die bei $300^{\circ} \mathrm{C}$ unter ansonsten identischen Bedingungen gesputterte $\mathrm{IrO}_{2}$ Schicht eine deutlich geringere Dichte von $\rho=10,9 \mathrm{~g} / \mathrm{cm}^{3}$, für eine Substrattemperatur von $100^{\circ} \mathrm{C}$ eine noch niedrigere Dichte von $\rho=10,8 \mathrm{~g} / \mathrm{cm}^{3}$. Es ist also für stöchiometrische $\mathrm{IrO}_{2}$ Schichten eine mit zunehmender Substrattemperatur $\mathrm{T}_{S}$ unter sonst konstant gehaltenen Parametern ansteigende Dichte festzustellen. Diese Zunahme wird auf eine während der Deposition thermisch aktivierte Oberflächendiffusion zurückgeführt, die ein Ausheilen von Leerstellen und Poren ermöglicht. Dies führt bei den $\mathrm{IrO}_{2}$ Schichten zu einer dichteren und kompakteren Mikrostruktur [Smi95], die für einen möglichst niedrigen diffusiven Materialtransport durch die Barrierenschicht anzustreben ist. Die vollständigen Daten zur Temperaturabhängigkeit sind in Tab. 4.1 zusammengefasst, zusätzlich ist die relative Differenz der Dichten zur $\mathrm{IrO}_{2}$-Referenz und die Rauigkeit der Schichten angegeben [JCP95]. Die für die Integration relevante Schichtrauigkeit wird im nachfolgenden Kapitel mit den Daten aus der Rastertunnelmikroskopie verglichen.

Tab. 4.1: Ergebnisse der Röntgen-Reflektometrieanalysen

\begin{tabular}{lllll}
\hline Depositionstemperatur & Schichtdicke & $\begin{array}{l}\text { Dichte aus } \\
\text { XRR-Fits }\end{array}$ & $\begin{array}{l}\text { Relative Differenz } \\
100^{*}\left(\rho_{\mathrm{XRR}}-\rho_{\mathrm{m}}\right) / \rho_{\mathrm{m}}[\%]\end{array}$ & $\begin{array}{l}\text { Rauigkeit } \\
\mathrm{T}_{\mathrm{S}}\left[{ }^{\circ} \mathrm{C}\right]\end{array}$ \\
\hline $\mathrm{d}[\mathrm{nm}]$ & $\rho_{\mathrm{XRR}}\left[\mathrm{g} / \mathrm{cm}^{3}\right]$ & & \\
\hline 100 & 91,9 & 10,8 & $-6,9$ & 1,5 \\
300 & 91,2 & 10,9 & $-6,0$ & 2,2 \\
500 & 91,0 & 11,3 & $-2,6$ & 2,0 \\
\hline
\end{tabular}

Eine systematische Analyse der Schichtdichte in Abhängigkeit des Sauerstoffpartialdrucks, die Abb. 4.11 zeigt, verdeutlicht den Einfluss des Sauerstoffpartialdrucks auf die Schichteigenschaften. Dabei wurde der Depositionsmodus mit reduzierter Pumpleistung $\left(\mathrm{s}_{\mathrm{p}}=70 \mathrm{l} / \mathrm{s}\right)$ gewählt, um eine möglichst breite Variation des Sauerstoffpartialdrucks zu realisieren. Anhand Abb. 4.11 ist wegen der stark unterschiedlichen Dichten des Iridiums und des Iridiumdioxids eine deutliche Dichtenabnahme innerhalb eines schmalen SauerstoffPartialdruckbereichs zu erkennen. Für die gesputterte Ir Schicht zeigt sich, dass die an der Schicht röntgenographisch bestimmte Dichte bei einer Substrattemperatur von $T_{S}=100^{\circ} \mathrm{C}$ etwa 7\% unter der Dichte von Massivproben liegt. An dem Punkt des maximalen SauerstoffGetterflusses $\mathrm{Q}_{\mathrm{S} \text {,max }}$ misst man für die stöchiometrischen $\mathrm{IrO}_{2}$ Schichten ebenfalls eine gegenüber Massivproben etwa um den gleichen Prozentsatz verminderte Dichte. Für noch höhere Sauerstoffpartialdrücke wird eine weitere kontinuierliche Abnahme der Dichte beobach- 
tet. Dieses Ergebnis kann aufgrund der extrem feinkörnigen Mikrostruktur der Ir und $\mathrm{IrO}_{2}$ Schichten mit Korngrößen von d=5-10 nm durch eine im Vergleich zum Festkörper veränderte Korngrenzenstruktur oder -dichte gedeutet werden. Zudem kann die zu hohen Sauerstoffpartialdrücken verminderte Dichte des $\mathrm{IrO}_{2}$ durch die mittels XRD nachgewiesene kristalline Unordnung und eine im Vergleich zum thermodynamischen Gleichgewichtswert erhöhten Leerstellenkonzentration auf dem Kationengitter gedeutet werden. Für sehr hohe Sauerstoffpartialdrücke wird eine Mikrostruktur vermutet, die aufgrund der gefundenen Sauerstoffüberstöchiometrie zunehmend porös wird und sogar Gaseinschlüsse vermuten lässt (vgl. Kap. 5.1.

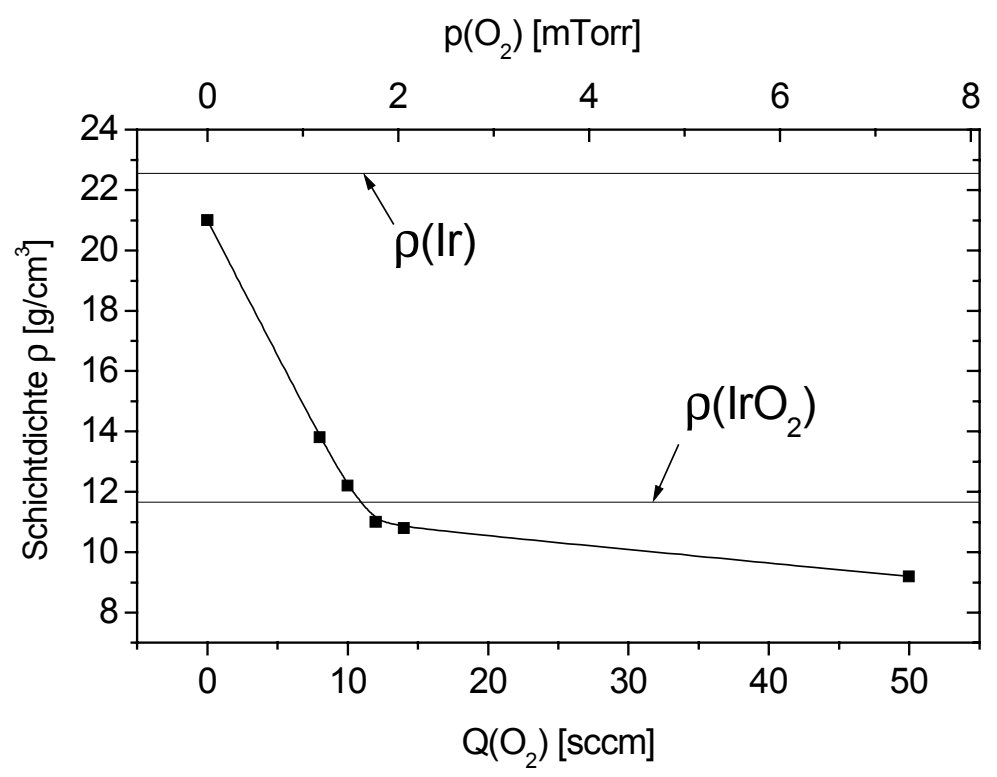

Abb. 4.11: Dichten der bei $100^{\circ} \mathrm{C}$ gesputterten $\mathrm{IrO}_{x}$ Schichten in Abhängigkeit des Sauerstoffpartialdrucks, die nominelle Dicke der Schichten beträgt $d=100 \mathrm{~nm}$. Als Substrat wurden thermisch oxidierte Si-Wafer benutzt, $\mathrm{d}\left(\mathrm{SiO}_{2}\right)=625 \mathrm{~nm}$. Die Linie ist zur Führung des Auges eingezeichnet. Als Referenz ist jeweils die in der Literatur angegebene Dichte von Ir und $\mathrm{IrO}_{2}$ eingezeichnet [Gme78]

Eine mit zunehmendem Sauerstoffpartialdruck und Sauerstoffübersättigung abnehmende Dichte wurde ebenfalls von Choi et al. [Cho01] bei der reaktiven Sputterdeposition von Indium-Zinn-Oxid (ITO) Schichten gefunden. Es konnte in diesem System ebenfalls gezeigt werden, dass die Dichte der Schichten deutlich mit den elektrischen Eigenschaften und dem residualen Schichtspannungen korrelieren und daher als wichtige Messgröße für reaktiv gesputterte Schichten angesehen werden kann. Eine detaillierte Analyse der mechanischen Spannungen und des elektrischen Widerstandes der Schichten in Abhängigkeit des Sauerstoffpartialdrucks während der Deposition wird im folgenden Kapitel durchgeführt. 


\subsection{Analyse der mechanischen Spannungen und Leitfähigkeitsmessungen}

Für die Leitfähigkeitsmessungen und die Messungen der mechanischen Schichtspannungen lässt sich wie in der Diskussion der Weitwinkelbeugungsspektren eine Einordnung der Schichteigenschaften in drei Regionen vornehmen. Die für eine Substrattemperatur von $100^{\circ} \mathrm{C}$ und $500^{\circ} \mathrm{C}$ gemessenen Ergebnisse sind in Abb. 4.12 dargestellt.
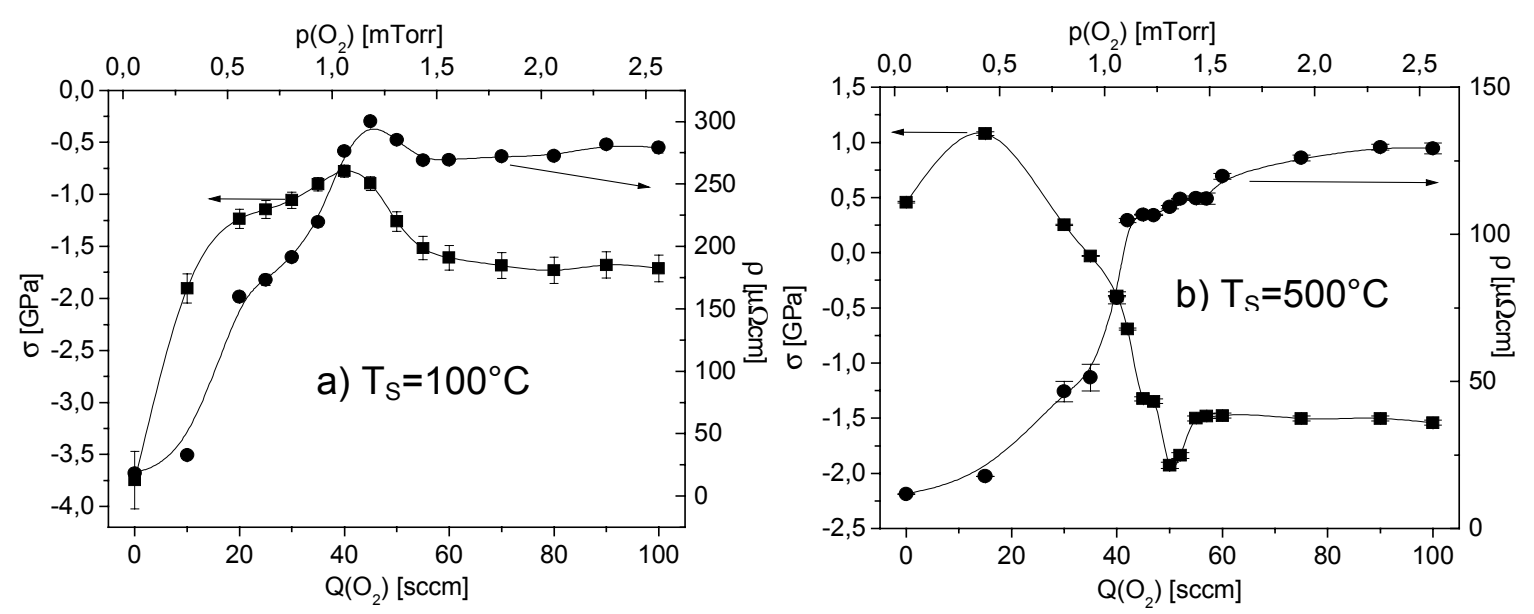

Abb. 4.12: Mechanische Schichtspannungen und spezifische Schichtwiderstände in Abhängigkeit des Sauerstoffpartialdrucks für eine Depositionstemperatur von a) $100^{\circ} \mathrm{C}$ und b) $500^{\circ} \mathrm{C}$ bei einer nominellen Schichtdicke von $\mathrm{d}=100 \mathrm{~nm}$. Als Substrat wurde thermisch oxidiertes $\mathrm{Si}$ verwendet. Die Depositionsleistung betrug $\mathrm{P}=300 \mathrm{~W}$, der Argonpartialdruck $p(\operatorname{Ar})=2,5 \mathrm{mTorr}$. Die Linien dienen zur Führung des Auges.

Bei einer Substrattemperatur von $100^{\circ} \mathrm{C}$ und nicht reaktiver Abscheidung zeigen die Ir Schichten sehr stark kompressive Spannungen von $\sigma=-3,7 \mathrm{GPa}$, die allerdings mit zunehmendem Reaktivgaspartialdruck in Region 1 deutlich abnehmen. Der Schichtwiderstand steigt von etwa $16 \mu \Omega \mathrm{cm}$ für reines Ir auf über $300 \mu \Omega \mathrm{cm}$. In Region 2, in der nach den Röntgenbeugungsanalysen die beste Kristallinität der $\mathrm{IrO}_{2}$ Phase beobachtet wird, nehmen die kompressiven Spannungen wieder auf einen konstanten Wert von etwa $\sigma=-1,6 \mathrm{GPa}$ zu, der spezifische Widerstand sinkt auf etwas unter $300 \mu \Omega \mathrm{cm}$ ab. Vergleichbare Widerstandswerte für bei Raumtemperatur gesputtertes $\mathrm{IrO}_{2}$ wurden von Horng et al. [Hor99] bzw. für bei $100^{\circ} \mathrm{C}$ gesputtertes $\mathrm{IrO}_{2}$ von Liao et al. [Lia98] gefunden.

Die bei einer Substrattemperatur von $500^{\circ} \mathrm{C}$ hergestellten Ir Schichten weisen leicht tensile Spannungen auf, die man aufgrund der differentiellen thermischen Ausdehnung zum SiSubstrat auch erwarten würde, wenn die Schichten bei der Deposition bei $500^{\circ} \mathrm{C}$ durch die Adatommobilität vollständig relaxiert aufwachsen [Gme78]. Für höhere Sauerstoffpartialdrücke in Region 2 werden aber wieder kompressive Spannungen beobachtet, die in dem Bereich der besten Kristallinität und des abrupten Texturumschlags maximal werden, für weiter 
erhöhte Drücke nahezu konstant bei $\sigma=-1,5 G P a$ bleiben. Die Spannungen der bei einer Substrattemperatur von $500^{\circ} \mathrm{C}$ hergestellten Schichten sind für die Region 2 nahezu identisch mit denen für die Abscheidung bei $100^{\circ} \mathrm{C}$. Der Schichtwiderstand der Ir Schichten ist für die gewählte Depositionstemperatur von $500^{\circ} \mathrm{C}$ mit $12 \mu \Omega \mathrm{cm}$ nur geringfügig niedriger als bei $100^{\circ} \mathrm{C}$ und steigt in Abb. 4.12 b) bei der $\mathrm{IrO}_{2}$ Phasenbildung schlagartig, in Region 2 dann schwach auf Werte von etwa $130 \mu \Omega \mathrm{cm}$ an. Für die in Abb. 4.12 nicht gezeigten, deutlich höheren Sauerstoffpartialdrücke in Region 3 sinken die kompressiven Spannungen und steigt der spezifische Widerstand monoton an, während die Kristallinität abnimmt.

Die Leitfähigkeit der Ir Schichten zeigt nur eine geringe Abhängigkeit von der Depositionstemperatur, was auf einen relativ geringen Einfluss der Defektdichte auf den elektrischen Widerstand hindeutet. Der Anstieg in Region 1 wird auf das Absinken der Kristallinität der Proben und damit auf eine verringerte freie Weglänge der Ladungsträger zurückgeführt. Das in Region 2 beobachtete Absinken des Schichtwiderstandes bei einer Substrattemperatur von $100^{\circ} \mathrm{C}$ kann mit einer Zunahme der $\mathrm{IrO}_{2}$ Korngrößen und einer damit verbundenen Reduktion der Ladungsträgerstreuung an Korngrenzen und $\mathrm{Ir} / \mathrm{IrO} \mathrm{I}_{2}$ Phasengrenzen in Region 2 erklärt werden. Das zunehmende Bombardement der Schicht durch Sauerstoffatome sowie das Übersättigen der $\mathrm{IrO}_{2}$ Phase mit Sauerstoff resultiert in einer Abnahme der kristallinen Ordnung und führt zu einer Schichtwiderstandserhöhung. In Übereinstimmung konnte mit XRD nachgewiesen werden, dass die mittlere Korngröße kontinuierlich mit zunehmendem Sauerstoffpartialdruck abnimmt.

Die gefundene Abhängigkeit der Spannungen vom Sauerstoffpartialdruck zeigt, dass die Größe der kompressiven Spannungen der Ir Schichten signifikant durch die Substrattemperatur beeinflusst wird, die Spannungen der $\mathrm{IrO}_{\mathrm{x}}$ Schichten nahezu unabhängig davon ist. Die gemessene Schichtspannung $\sigma$ lässt sich additiv in einen thermischen $\sigma_{t h}$ und einen intrinsischen Anteil $\sigma_{\text {in }}$ gemäß

$\sigma=\sigma_{t h}+\sigma_{\text {in }}$

zerlegen [Cho01]. Aufgrund der Ergebnisse der Röntgenweitwinkelbeugung werden die starken kompressiven Spannungen des Ir und besonders des IrO $_{x}$ auf den kinetischen Beschuss zurückgeführt, der sich in einer nachweisbaren Gitterdilatation äußert. Bei einer niedrigen Substrattemperatur ist sowohl für die reine Ir Deposition als auch für die reaktive $\mathrm{IrO}_{\mathrm{x}}$ Deposition der intrinsische Spannungsanteil dominierend; die kompressiven Spannungen werden im Fall der Ir Deposition auf den Argonbeschuss, im Fall der reaktiven Deposition auf den Argon- und den Sauerstoffbeschuss zurückgeführt. Dabei wird angenommen, dass aufgrund der geringeren Masse des Sauerstoffs dieser Beschuss als dominanter Mechanismus der Spannungserzeugung angesehen werden kann. Bei der niedrigen Substrattemperatur sind 
kaum Relaxationsprozesse thermisch aktiviert, die zu einer signifikanten Verringerung der Spannungen beitragen könnten. Damit werden Defekte durch das nachfolgend abgeschiedene Material weitestgehend eingefroren.

Die Tatsache, dass bei reiner Ir Sputterdeposition zwischen den zwei untersuchten Depositionstemperaturen eine Spannungsdifferenz von $\Delta \sigma=4,2 G P a$ vorliegt, deutet auf eine Spannungsrelaxation während des Wachstums bei $500^{\circ} \mathrm{C}$ hin, da aufgrund von nur differentieller thermischer Ausdehnung, die gemäß

$\sigma_{t h}=-\int_{T_{1}}^{T_{2}} B_{f} \cdot\left(\alpha_{f}-\alpha_{s}\right) d T$

ausgedrückt werden kann [Tho89], maximal etwa $\Delta \sigma=1,25 \mathrm{GPa}$ geringere kompressive Spannungen erwartet werden [Gme78]. Dabei stellt $B_{f}$ den Schichtbiaxialmodul, $\alpha_{f}$ und $\alpha_{s}$ die thermischen Ausdehnungskoeffizienten der Schicht und des Substrats dar. Daher wird angenommen, dass die durch den Argonbeschuss erzeugten, spannungsverursachenden Defekte im Ir bei einer Substrattemperatur von $500^{\circ} \mathrm{C}$ nahezu vollständig ausheilen können.

Für die $\mathrm{IrO}_{x}$-Deposition verhindert die durch die Depositionskinetik erzeugte hohe Defektdichte die Reduktion der Spannungen sogar bei der höchsten gewählten Depositionstemperatur von $500^{\circ} \mathrm{C}$, da die Defektdichte die Beweglichkeit von Versetzungen stark einschränkt. Die erhöhte atomare Oberflächenbeweglichkeit kann die erzeugten Defekte und Spannungen nicht kompensieren, da sich die Schädigung durch den ballistischen Beschuss nicht auf die oberste atomare Lage beschränkt, sondern sich generell mehrere Nanometer in die Tiefe der Schicht erstreckt [Beh81].

Zudem kann nachgewiesen werden, dass die kompressiven Spannungen mit der Kristallinität der $\mathrm{IrO}_{2}$ Proben korrelieren. Dieser Effekt ist in Abb. 4.12 b) bei einer Substrattemperatur von $500^{\circ} \mathrm{C}$ und Sauerstoffflüssen von etwa $\mathrm{Q}\left(\mathrm{O}_{2}\right)=50 \mathrm{sccm}$ nachweisbar. Für diesen Flussbereich sind die $\mathrm{IrO}_{2}$-Körner mit den größten Kohärenzlängen beobachtet worden, gleichzeitig zeigen diese Schichten die höchsten kompressiven Spannungen. Dieses Ergebnis kann so gedeutet werden, dass die spannungserzeugenden Zentren im Wesentlichen in den Körnern lokalisiert sind. Die in-situ Spannungsrelaxation der $\mathrm{IrO}_{2}$ Schichten während des Anlassens wird in Kap 5.4 in Abhängigkeit der gewählten Substrattemperatur im Detail untersucht.

\subsection{Oberflächenmorphologieanalysen mittels Rastertunnelmikroskopie (STM)}

Mittels Rastertunnelmikroskopie sind Oberflächenmorphologieanalysen an den $\mathrm{IrO}_{2}$ Schichten durchgeführt worden. Es existieren rasterkraftmikroskopische Analysen zu reaktiv 
gesputterten $\mathrm{IrO}_{2}$ Schichten von Liao et al. [Lia98], jedoch weisen die Ergebnisse Unstimmigkeiten zwischen den kraftmikroskopisch ermittelten Daten zu Korngrößen und denen aus der Röntgenbeugung auf, die nicht diskutiert werden. Aufgrund der guten Leitfähigkeit der Schichten und guten Qualität der mechanisch gezogenen STM-Spitzen sollen hier die Ergebnisse der rastertunnelmikroskopischen Analysen vorgestellt und mit den XRD-Daten verglichen werden.

Anhand der STM-Daten wurde die Oberflächenrauigkeit und die mittlere Korngröße der Schichten in Abhängigkeit von der Substrattemperatur und des Sauerstoffpartialdrucks untersucht. Die mittlere quadratische Rauigkeit ist als

$$
\delta^{2}=\left\langle S(x, y)^{2}\right\rangle-\langle S(x, y)\rangle^{2}=\left\langle(S(x, y)-\langle S(x, y)\rangle)^{2}\right\rangle
$$

definiert, wobei $S(x, y)$ die z-Koordinate des Oberflächenprofils darstellt und die eckigen Klammern für die geometrische Mittelung stehen. Die mittlere Korngröße wurde durch Anpassen einer Gaußfunktion an die azimutal gemittelte Autokorrelationsfunktion des zweidimensionalen STM-Bildes bestimmt [Ras82], [Ras83], [Ras84]. Die Autokorrelationsfunktion

$$
G\left(x^{\prime}, y^{\prime}\right)=\left\langle H(x, y) \cdot H\left(x+x^{\prime}, y+y^{\prime}\right)\right\rangle
$$

stellt eine Höhen-Höhen-Korrelation dar, wobei die Funktion $H(x, y)$ durch

$$
H(x, y)=S(x, y)-\langle S(x, y)\rangle
$$

definiert ist und die Profilhöhe in Bezug auf die mittlere Ebene $\langle S(x, y)\rangle$ darstellt. Die Rauigkeit und die mittlere Korngröße wurden aus mehreren STM-Aufnahmen bestimmt, die eine für die Schicht statistisch repräsentative Vielzahl von Körnern enthält. Die Dicke aller untersuchten Schichten ist etwa $100 \mathrm{~nm}$, als Substrate wurden thermisch oxidierte Si-Wafer verwendet. In Abb. 4.13 sind STM-Aufnahmen einer bei $500^{\circ} \mathrm{C}$ mit einem reaktiven Fluss von $\mathrm{Q}\left(\mathrm{O}_{2}\right)=60 \mathrm{sccm}$ im ungedrosselten Pumpmodus gesputterten, (200)-texturierten, reinphasigen stöchiometrischen $\mathrm{IrO}_{2}$ Schicht gezeigt. Die Aufnahmen haben Kantenlängen von a) $1 \mu \mathrm{m}$, b) $500 \mathrm{~nm}$ und c) $125 \mathrm{~nm}$ und zeigen ein sehr feinkörniges, isotropes Gefüge von dicht liegenden, teils nadelförmigen Strukturen. Als mittlere quadratische Rauigkeit ergibt sich für diese Schicht ein Wert von $\delta=(2,5 \pm 0,2) \mathrm{nm}$, der vergleichbar mit dem durch die Röntgenreflektometrie gefundenen ist. Als mittlere Korngröße erhält man aus dem Gaußfit der radiaIen Autokorrelationsfunktion einen Wert von $d=(10,3 \pm 2,0) \mathrm{nm}$. 


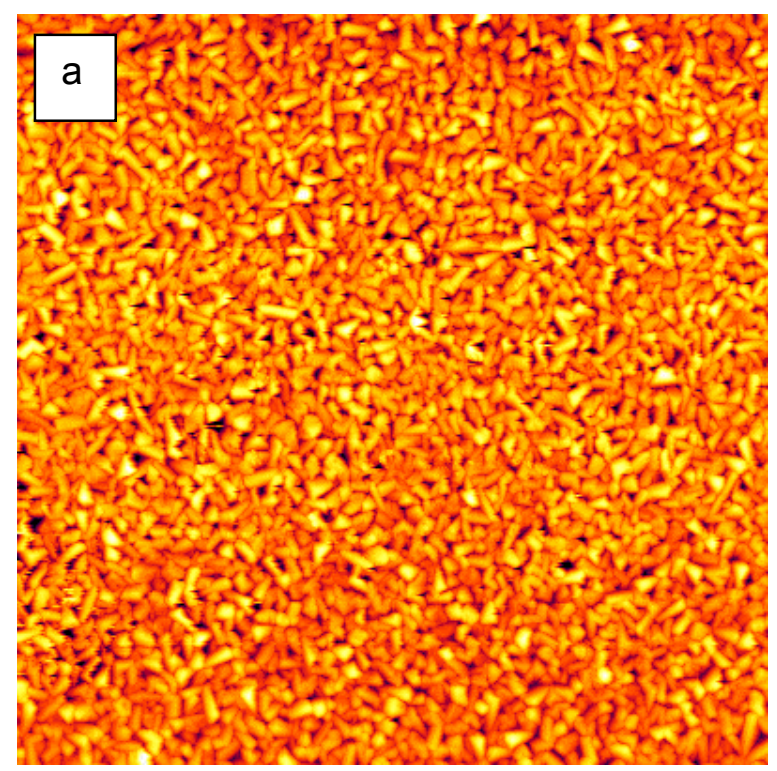

Abb. 4.13: STM-Aufnahme einer $\mathrm{IrO}_{2}$ Schicht, $\mathrm{T}_{\mathrm{S}}=500^{\circ} \mathrm{C}, \mathrm{d}\left(\mathrm{IrO}_{2}\right)=100 \mathrm{~nm}$ Kantenlänge $\mathrm{I}=1000 \mathrm{~nm}$
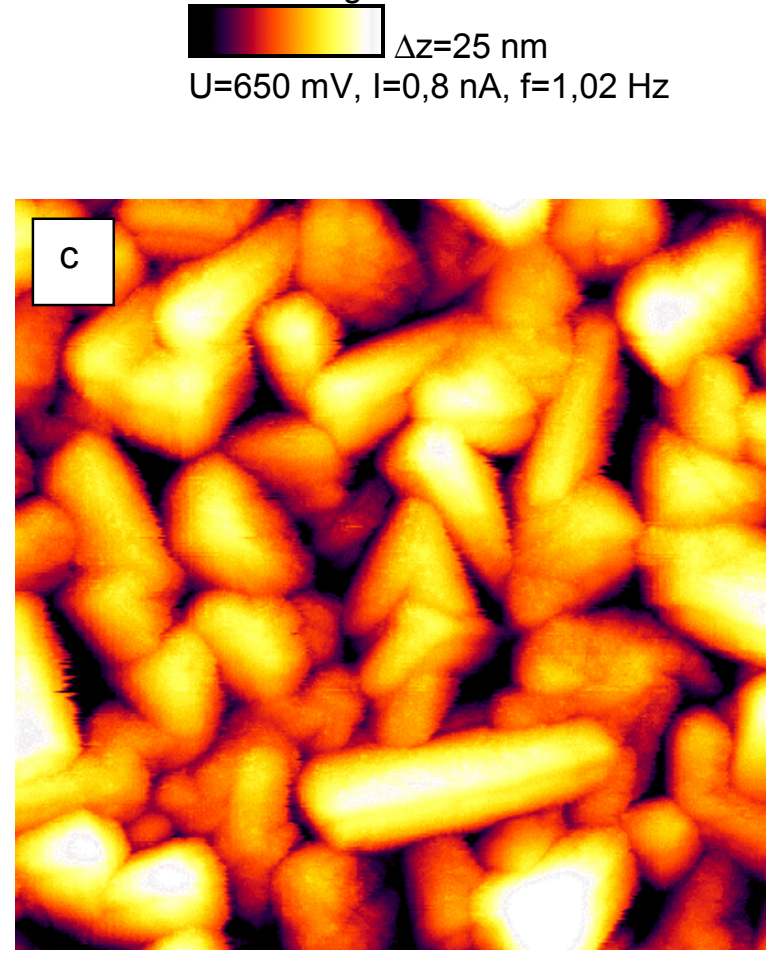

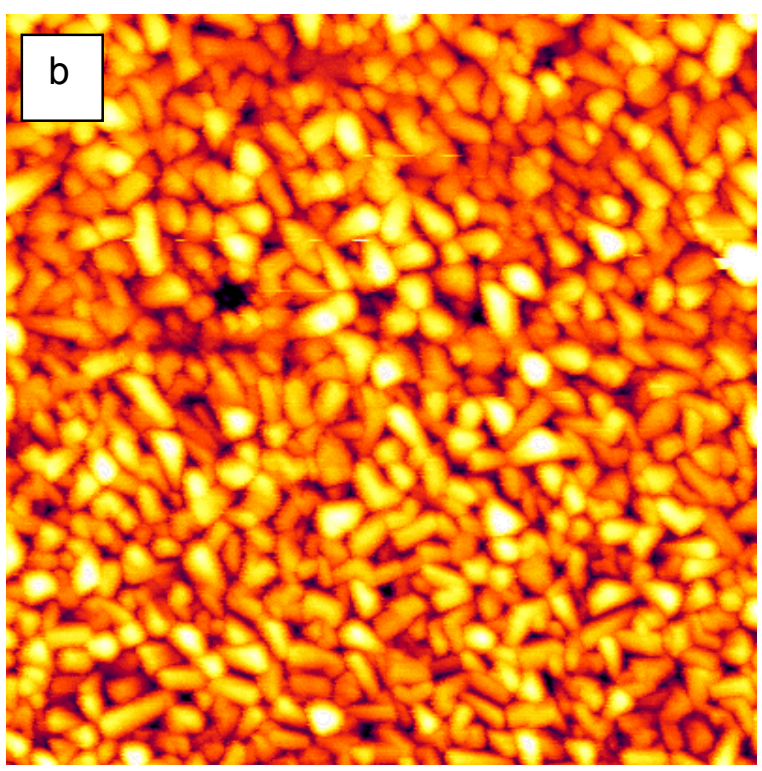

STM-Aufnahme einer $\mathrm{IrO}_{2}$ Schicht, $\mathrm{T}_{\mathrm{S}}=500^{\circ} \mathrm{C}, \mathrm{d}\left(\mathrm{IrO}_{2}\right)=100 \mathrm{~nm}$ Kantenlänge $\mathrm{l}=500 \mathrm{~nm}$

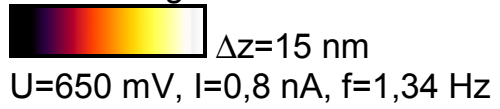

STM-Aufnahme einer $\mathrm{IrO}_{2}$ Schicht,

$\mathrm{T}_{\mathrm{S}}=500^{\circ} \mathrm{C}, \mathrm{d}=100 \mathrm{~nm}$

Kantenlänge $\mathrm{I}=125 \mathrm{~nm}$,

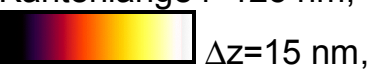

$\mathrm{U}=650 \mathrm{mV}, \mathrm{l}=0,8 \mathrm{nA}, \mathrm{f}=1,93 \mathrm{~Hz}$, (leichtes Spitzenartefakt)

Die in Abb. 4.14 gezeigten STM-Bilder sind bei einer $\mathrm{IrO}_{2}$ Schicht aufgenommen worden, die mit einer Substrattemperatur von $100^{\circ} \mathrm{C}$ unter ansonsten identischen Bedingungen hergestellt wurde. Es zeigt sich eine Oberflächenmorphologie, die keine charakteristisch nadelför- 
migen Oberflächenstrukturen aufweist, sondern einheitlich rundlich erscheinende Strukturen darstellt.

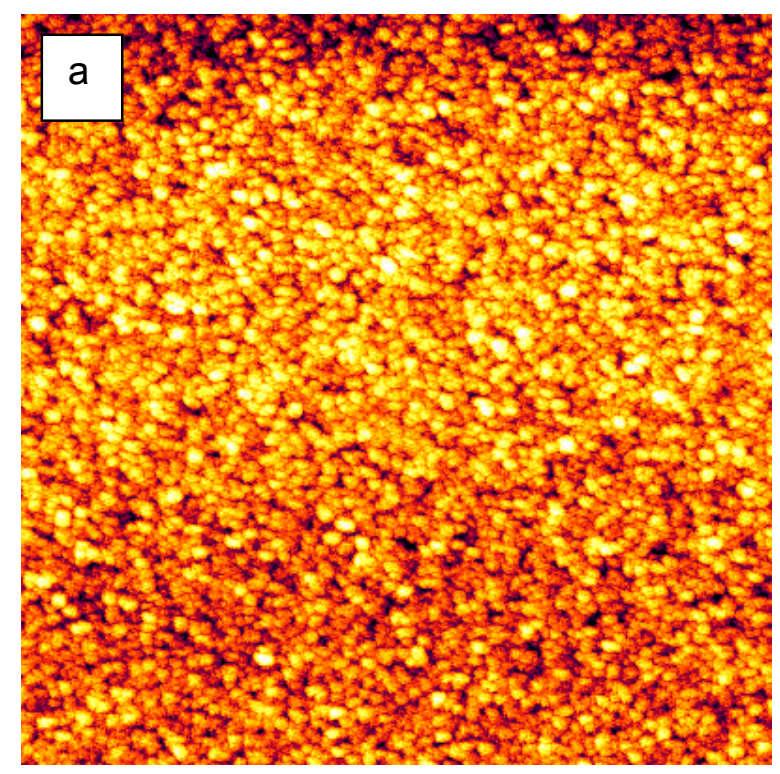

Abb. 4.14: STM-Bild einer $\operatorname{IrO}_{2}$ Schicht, $\mathrm{T}_{\mathrm{S}}=100^{\circ} \mathrm{C}, \mathrm{d}\left(\mathrm{IrO}_{2}\right)=100 \mathrm{~nm}$ Kantenlänge $\mathrm{l}=1000 \mathrm{~nm}$ $\mathrm{U}=650 \mathrm{mV}, \mathrm{I}=0,8 \mathrm{nA}, \mathrm{f}=1,34 \mathrm{~Hz}$

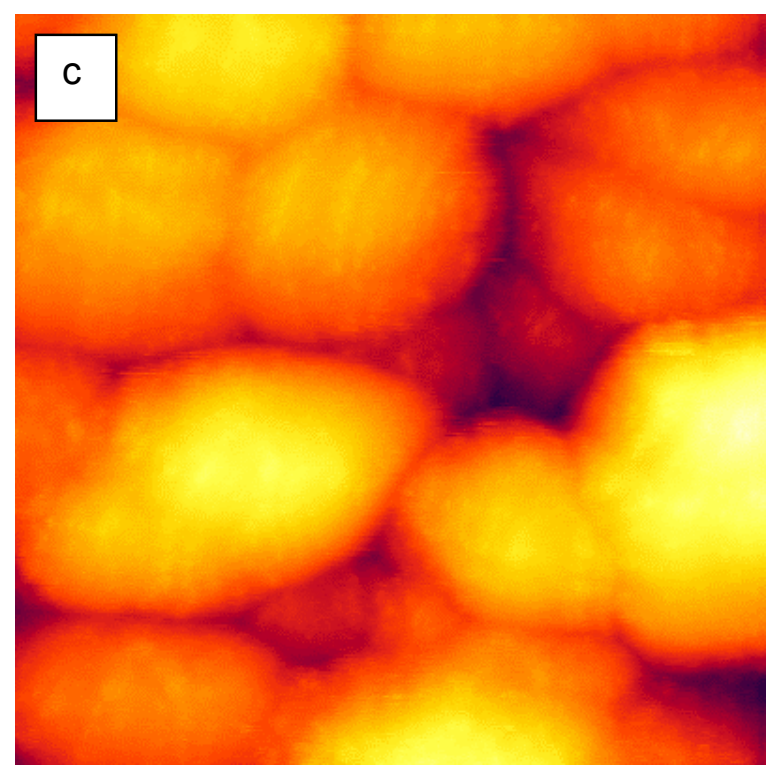

STM-Bild einer $\mathrm{IrO}_{2}$ Schicht $\mathrm{T}_{\mathrm{S}}=100^{\circ} \mathrm{C}, \mathrm{d}\left(\mathrm{IrO}_{2}\right)=100 \mathrm{~nm}$ Kantenlänge $\mathrm{l}=50 \mathrm{~nm}$

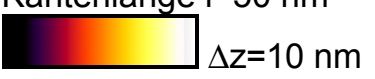

$\mathrm{U}=650 \mathrm{mV}, \mathrm{I}=0,8 \mathrm{nA}, \mathrm{f}=4,34 \mathrm{~Hz}$

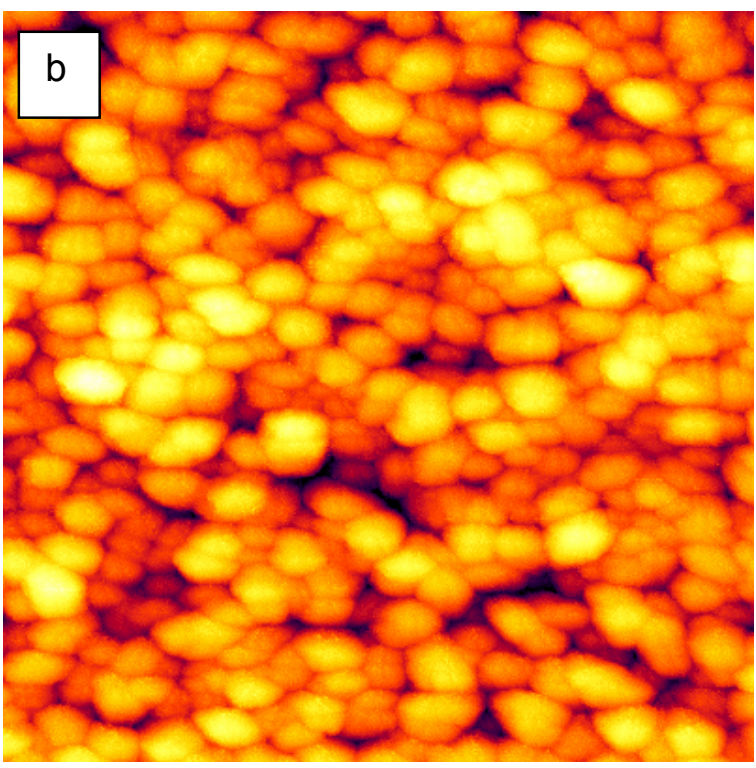

STM-Bild einer $\mathrm{IrO}_{2}$ Schicht, $\mathrm{T}_{\mathrm{S}}=100^{\circ} \mathrm{C}, \mathrm{d}\left(\mathrm{IrO}_{2}\right)=100 \mathrm{~nm}$ Kantenlänge $\mathrm{I}=250 \mathrm{~nm}$

$\mathrm{U}=650 \mathrm{mV}, \mathrm{I}=0,8 \mathrm{nA}, \mathrm{f}=1,34 \mathrm{~Hz}$


Den in Abb. 4.14 gezeigten Strukturen fehlen die typischen kristallographischen Kanten, wie sie in den bei $500^{\circ} \mathrm{C}$ deponierten $\mathrm{IrO}_{2}$ Schichten deutlich zu erkennen sind. Dieses Resultat deutet auf eine geringe Oberflächendiffusion während der Deposition und auf geringe Rekonstruktion hin. Die mittlere Größe dieser runden Strukturen ist jedoch vergleichbar mit denen der bei $500^{\circ} \mathrm{C}$ hergestellten Probe und beträgt $d=(11,3 \pm 3,2) \mathrm{nm}$. Die Schicht weist eine niedrige Rauigkeit von $\delta=(1,4 \pm 0,1) \mathrm{nm}$ auf, die innerhalb des Fehlers mit der durch die Röntgenreflektometrie gefundenen mittleren quadratischen Rauigkeit identisch ist.

Für die Abscheidetemperatur von $500^{\circ} \mathrm{C}$ wurden weitere systematische Untersuchungen der Korngrößen und Oberflächenmorphologie in Abhängigkeit des zur Deposition benutzten Sauerstoffpartialdrucks durchgeführt, um den Einfluss des Sauerstoffpartialdrucks auf die Schichtmorphologie zu untersuchen. Dabei stellt sich heraus, dass die körnige Oberflächenmorphologie mit zunehmendem Sauerstoffpartialdruck deutlich dichter wird und gleichzeitig die Rauigkeit kontinuierlich abnimmt, von $\delta=4,3 \mathrm{~nm}$ für die in Abb. 4.15 a) gezeigte Schicht auf $\delta=2,1 \mathrm{~nm}(\mathrm{Abb} . \mathbf{4 . 1 5} \mathrm{c})$ ).
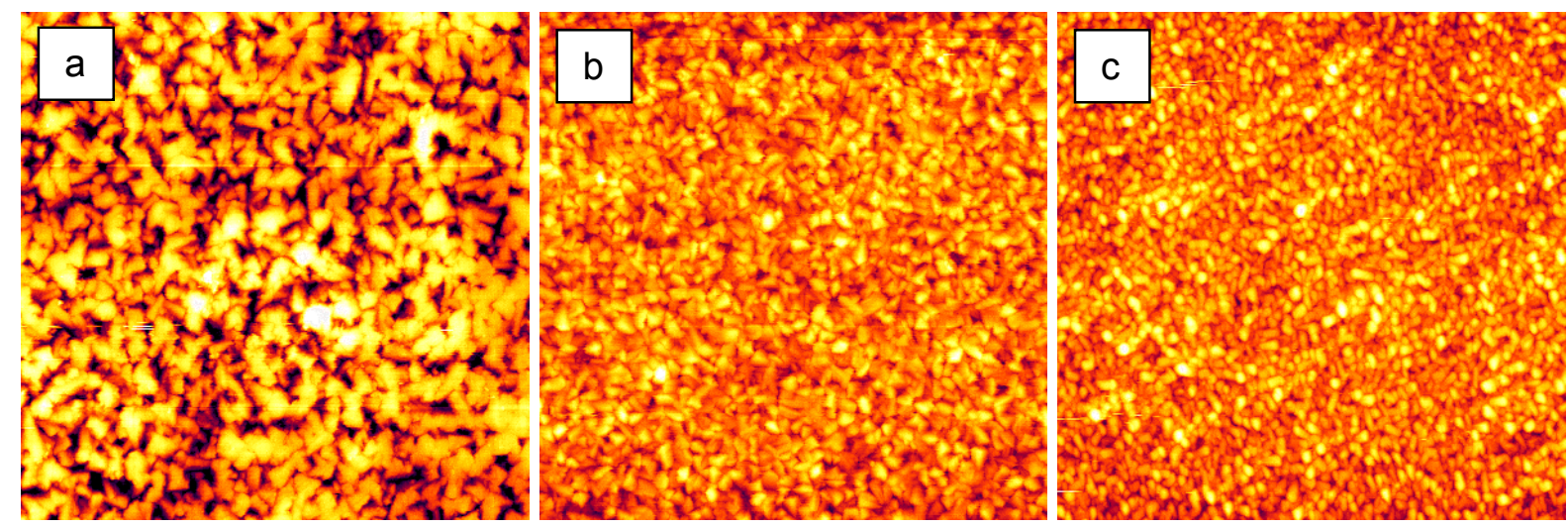

Abb. 4.15: STM-Bilder der $\operatorname{IrO} \mathrm{X}_{x}$ Schichten mit zunehmendem Sauerstoffpartialdruck, $\mathrm{T}_{S}=500^{\circ} \mathrm{C}$, $\mathrm{d}\left(\mathrm{IrO} \mathrm{O}_{2}\right)=100 \mathrm{~nm}$, Kantenlänge jeweils $\mathrm{I}=1000 \mathrm{~nm}$, a) $\Delta \mathrm{z}=25 \mathrm{~nm}, Q\left(\mathrm{O}_{2}\right)=42 \mathrm{sccm}$, b) $\left.\Delta \mathrm{z}=25 \mathrm{~nm}, \mathrm{Q}\left(\mathrm{O}_{2}\right)=52 \mathrm{sccm}, \mathrm{c}\right) \Delta \mathrm{z}=20 \mathrm{~nm}, \mathrm{Q}\left(\mathrm{O}_{2}\right)=90 \mathrm{sccm}$

Die in Abb. 4.16 dargestellten Ergebnisse über die Korngrößen sind aus den Analysen der STM-Bilder ermittelt worden, zusätzlich sind die Röntgendiffraktometrie-Spektren der Proben mittels Scherrerformel ausgewertet worden. Die Daten zu den Korngrößen zeigen, dass bei der untersuchten Abscheidetemperatur für geringe Sauerstoffpartialdrücke die Größe der (110)-orientierten Körner dominiert, für größere Sauerstoffpartialdrücke sich das Verhältnis zugunsten der (200)-orientierten Körner ändert. Es ist also eine klare Korrelation zwischen Korngröße und Textur gegeben, die zeigt, dass (110)-orientierte Körner bevorzugt bei nicht zu hohem Sauerstoffpartialdruck wachsen. 


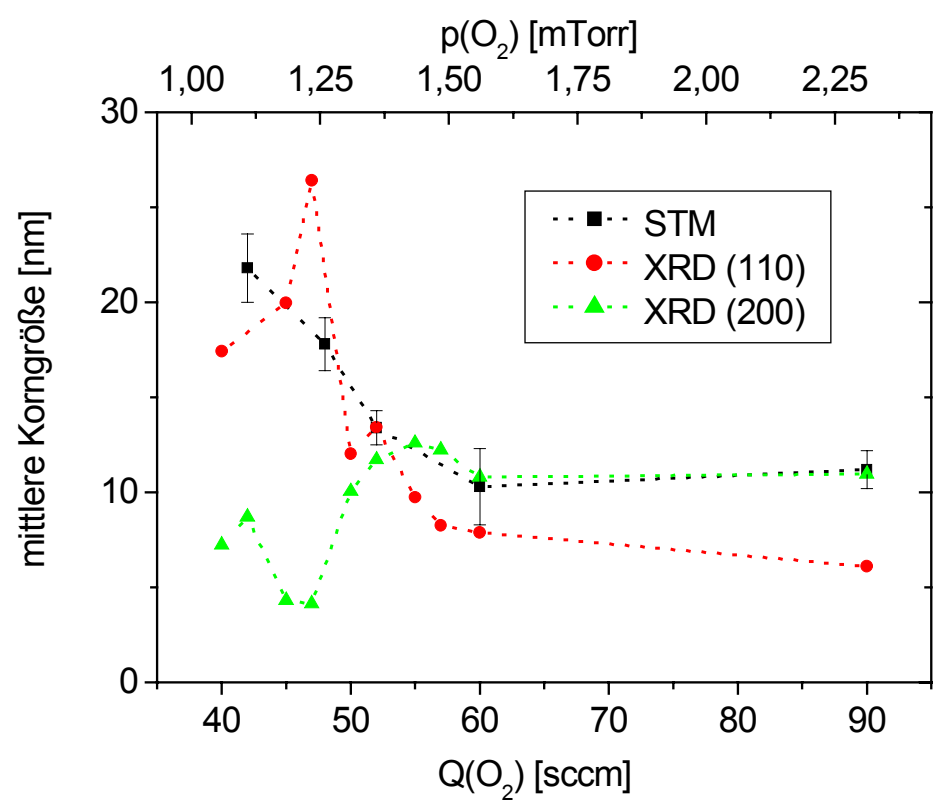

Abb. 4.16: Vergleich der lateralen Strukturgrößen der STM-Daten mit den Korngrößen aus der Scherrer-Abschätzung (XRD) aus den angegebenen Bragg-Reflexen für eine Depositionstemperatur von $500^{\circ} \mathrm{C}$

Es gibt bei einem Sauerstofffluss von knapp unter $Q\left(\mathrm{O}_{2}\right)=50 \mathrm{sccm}$ neben dem Maximum der kompressiven Spannungen ein Optimum der Kristallinität mit einem in Abb. 4.16 deutlich erkennbaren Maximum in der Korngröße. Dieser Sauerstoffpartialdruck kann als optimale Kornwachstumsbedingung aufgefasst werden: Für geringere Sauerstoffpartialdrücke fehlt der nötige Sauerstoff für das stöchiometrische Ausbilden der $\mathrm{IrO}_{2}$ Phase, bei zunehmendem Sauerstoffpartialdruck wird der bereits diskutierte Sauerstoff-Ionenbeschuss signifikant und verhindert die bei dieser Depositionstemperatur bis zur Target-Belegung zu erwartende Verbesserung der Kristallinität. Der Punkt der Target-Belegung wurde in Kap 3.7 ermittelt und liegt für die benutzten Depositionsparameter bei einem Fluss von etwa $Q\left(\mathrm{O}_{2}\right)=60 \mathrm{sccm}$. Unter Berücksichtigung, dass die benutzte Auswertemethode der STM-Daten die Strukturgrößen der größeren Körner widerspiegelt, zeigt sich, dass die beiden Methoden vergleichbare Ergebnisse liefern. Da mit der STM-Analyse die Korngröße in der Schichtebene bestimmt wird, die aus den XRD Daten bestimmten Kohärenzlängen eine Abschätzung für die Korngröße in Schichtwachstumsrichtung, also senkrecht zur STM-Analyseebene gibt, kann aus der Übereinstimmung dieser Daten auf eine Isotropie der Korngröße in Schichtwachstumsrichtung und in der Schichtebene geschlossen werden. Eine detaillierte Analyse der Mikrostruktur des gesputterten $\mathrm{IrO}_{2}$ wird im folgenden Kapitel mittels Transmissionselektronenmikroskopie angestellt. 


\subsection{Transmissionselektronenmikroskopie-Analysen (TEM)}

Zur detaillierten Untersuchung der lateralen und vertikalen Mikrostruktur der reaktiv gesputterten $\mathrm{IrO}_{2}$ Schichten wurden Elektronenmikroskopie- und Elektronenbeugungs-Analysen angestellt. Dabei soll speziell geklärt werden, ob in den kristallinen Proben vertikale Diffusionspfade existieren, die die Ursache für einen möglichen Korngrenzendiffusionsmechanismus darstellen können. Abb. 4.17zeigt eine Hellfeldaufnahme einer bei $100^{\circ} \mathrm{C}$ gesputterten, stöchiometrischen $\mathrm{IrO}_{2}$ Schicht. Deutlich als dunkle Flecken zu erkennen sind die etwa 5-15 nm großen Körner, die mittels der in Abb. 4.18 gezeigten Elektronenbeugung eindeutig der tetragonalen $\mathrm{IrO}_{2}$ Phase zugeordnet werden.

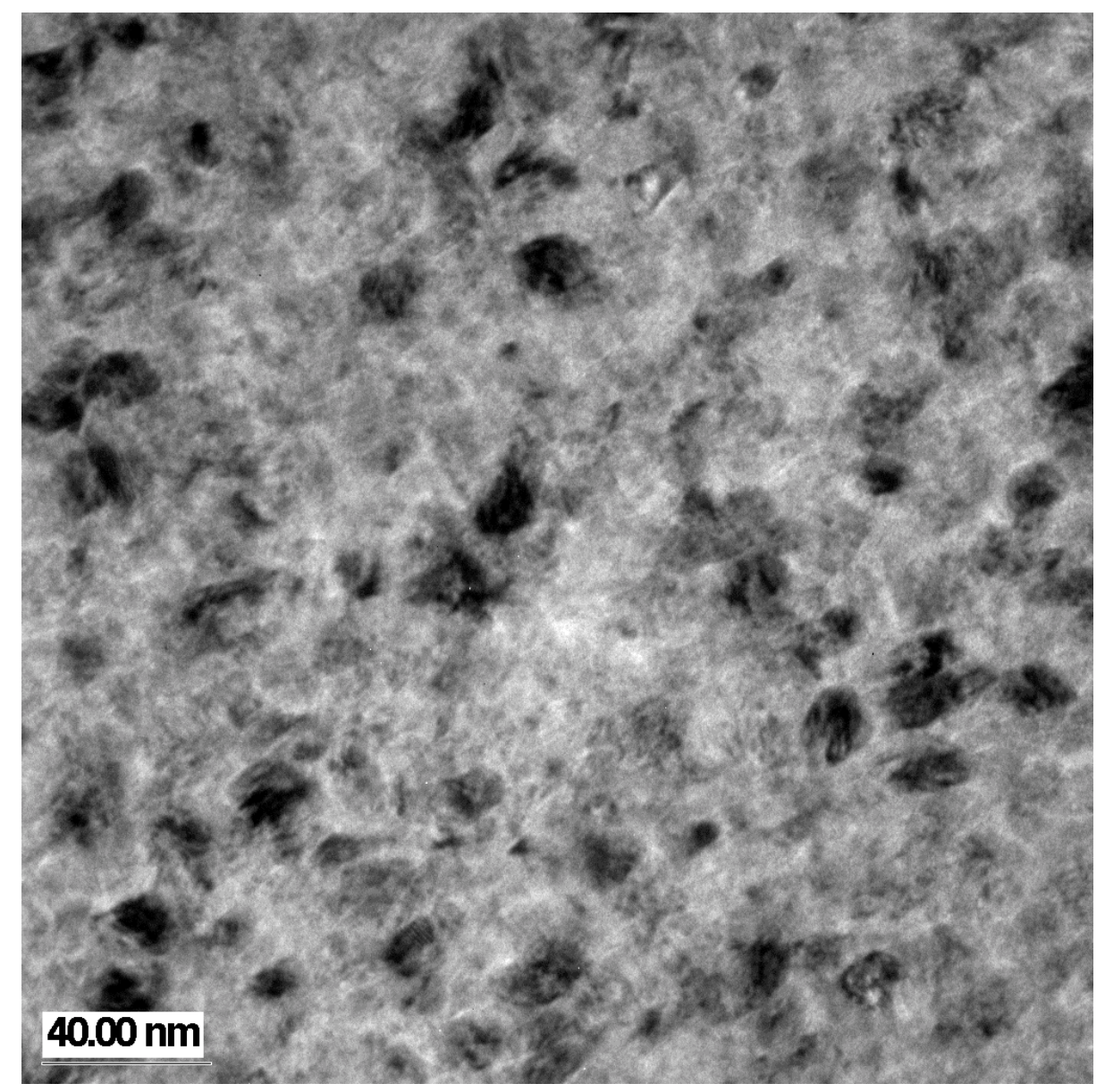

Abb. 4.17: $\mathrm{TEM}$ Hellfeldaufnahme der auf $\mathrm{a}-\mathrm{SiO}_{2}$ bei $100^{\circ} \mathrm{C}$ deponierten, stöchiometrischen $\mathrm{IrO}_{2}$ Schicht in Schichtebene. Die Sputterleistung betrug P=300 W, der Argon-Partialdruck betrug $\mathrm{p}(\mathrm{Ar})=2,5 \mathrm{mTorr}$. (60.000-fache Vergrößerung) 


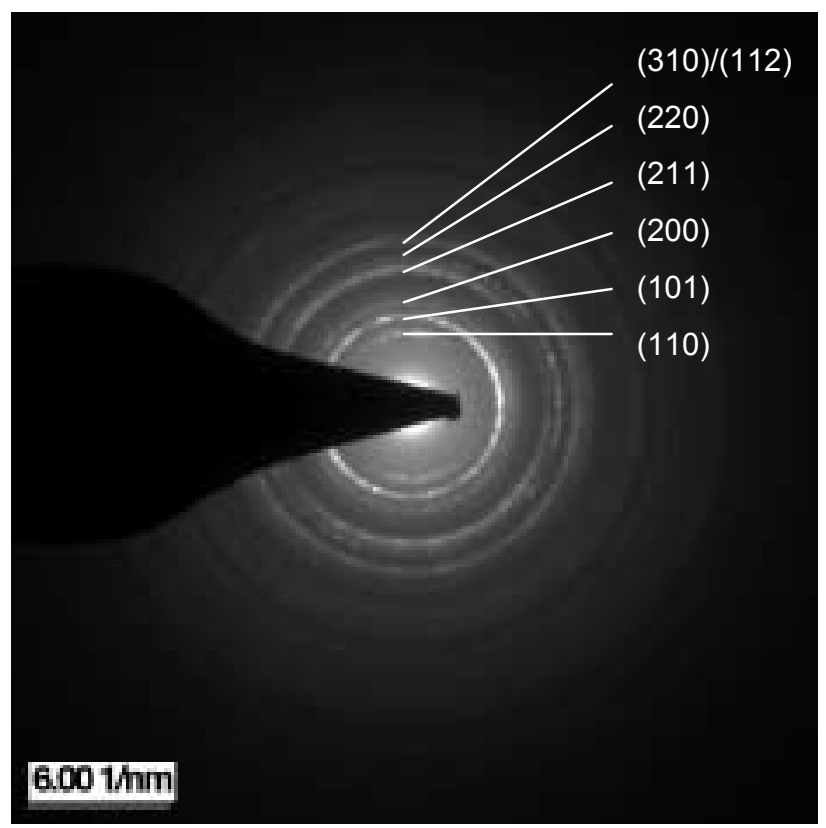

Abb. 4.18: Elektronenbeugungsbild der bei $100^{\circ} \mathrm{C}$ deponierten stöchiometrischen $\mathrm{IrO}_{2}$ Schicht, $\mathrm{P}=300 \mathrm{~W}, \mathrm{p}(\mathrm{Ar})=2,5 \mathrm{mTorr}$. Die Indizierung der Beugungsringe der tetragonalen $\mathrm{IrO}_{2}$ Phase ist angegeben.

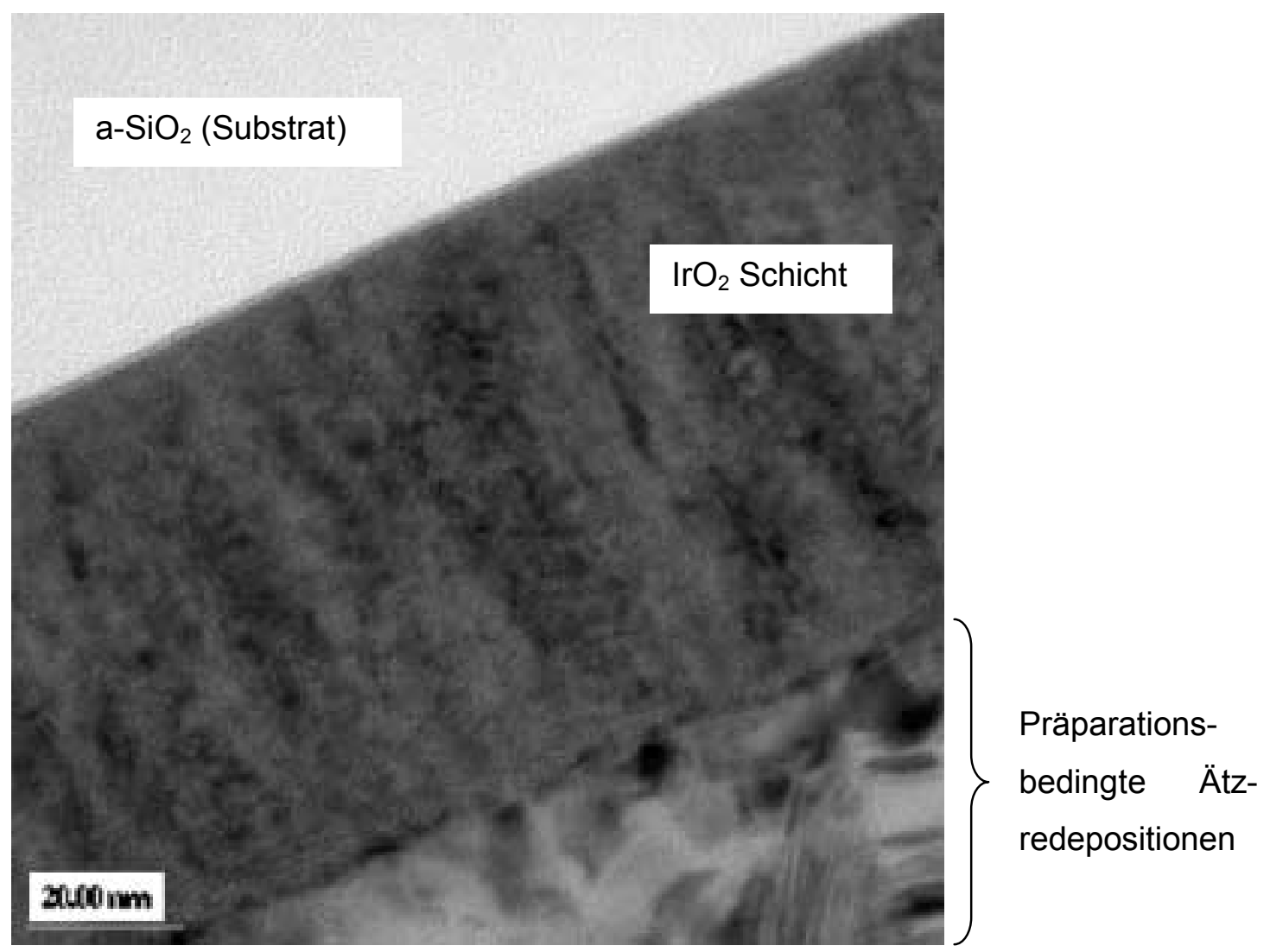

Abb. 4.19: TEM Hellfeldaufnahme der bei $100^{\circ} \mathrm{C}$ auf a- $\mathrm{SiO}_{2}$ deponierten stöchiometrischen $\mathrm{IrO}_{2}$ Probe im Querschliff. Die Sputterleistung betrug $\mathrm{P}=300 \mathrm{~W}$, der zur Deposition verwendete Argon-Partialdruck betrug $\mathrm{p}(\mathrm{Ar})=2,5 \mathrm{mTorr}$. 
In Abb. 4.19 ist eine Hellfeld-Querschliffanalyse derselben $\mathrm{IrO}_{2}$ Probe gezeigt, in der man oben links als hellen Bereich das sich auf dem Substrat befindende amorphe $\mathrm{SiO}_{2}$ erkennt, auf dem eine faserförmig, kolumnare $\mathrm{IrO}_{2}$ Schicht aufgewachsen ist. Die unregelmäßig helIen und dunklen Bereiche im Bild rechts unten sind durch den QuerschliffPräparationsprozess verursachte Verunreinigungen der Schichtoberseite. Dieses polykristalline Material wird mittels EDX-Analyse als vom Probenträger der lonenätzanlage stammendes Kupfer identifiziert und hat nichts mit der eigentlichen $\mathrm{IrO}_{2}$ Schicht zu tun. Die stark gestörte, kolumnare Struktur der Schicht, die gleichzeitig jedoch keine vertikal verlaufenden Pfade geringerer Dichte erkennen lässt, deutet auf eine Schichtmorphologie hin, die von Thornton [Tho86], [Smi95] als Zone-T Struktur ${ }^{60}$ bezeichnet wird. Diese Struktur wird charakteristischerweise bei einem Schichtwachstum beobachtet, das unter energetischem Teilchenbeschuss stattfindet, weiterhin typisch für das Auftreten einer solchen Struktur ist eine geringe reduzierte Depositionstemperatur ${ }^{61} T_{S} / T_{m}$ und damit eine niedrige Oberflächendiffusion. Die beobachtete Schichtmorphologie lässt für die verwendete Depositionstemperatur von $\mathrm{T}_{S}=100^{\circ} \mathrm{C}$ auf einen ballistischen Depositionsprozess schließen.

Anhand hochauflösender Mikroskopie an der Probe (Abb. 4.20) können trotz der faserförmigen Struktur der Schicht keine offenen Diffusionspfade bzw. Kanäle entlang von Korngrenzen oder Säulen nachgewiesen werden. Dieses Ergebnis ist konsistent mit den gefundenen hohen kompressiven Spannungen der stöchiometrischen $\mathrm{IrO}_{2}$ Schichten und der hohen Dichte, die nur wenige Prozent vom Volumenwert für $\mathrm{IrO}_{2}$ abweicht. Man erkennt aus der in Abb. 4.20 gezeigten hochauflösenden, und anderen hier nicht gezeigten Aufnahmen vielmehr, dass vermehrt starke Helligkeitskontraste innerhalb der Körner sichtbar sind, die lokalen Gitterdefekten zugeordnet werden. Zudem bilden sich häufig (110)-Zwillingsstrukturen aus, deren Zwillingsgrenzen in Schichtwachstumsrichtung zeigen. In Abb. 4.20 ist eine für den markierten Bildausschnitt berechnete, zweidimensionale Fouriertransformation (FFT) gezeigt, auf der vier (110)-Reflexe zu sehen sind, die nahezu spiegelsymmetrisch zur Schichtwachstumsrichtung angeordnet sind. Da die (110)- und (200)-Ebenen einen Winkel von $\alpha=45^{\circ}$ einschließen, liegen bei diesen Zwillingsstrukturen die (200)-Ebenen in der Schichtebene, was konsistent mit der beobachteten starken (200)-Textur dieser Schicht ist.

\footnotetext{
${ }^{60}$ Zone-T: Transition Zone (engl.) = Übergangsbereich

${ }^{61}$ als reduzierte oder homologe Temperatur wird die auf den Schmelzpunkt des Schichtmaterials $T_{m}$ normierte Depositionstemperatur $T_{S}$ bezeichnet, also $T_{S} / T_{m}$
} 


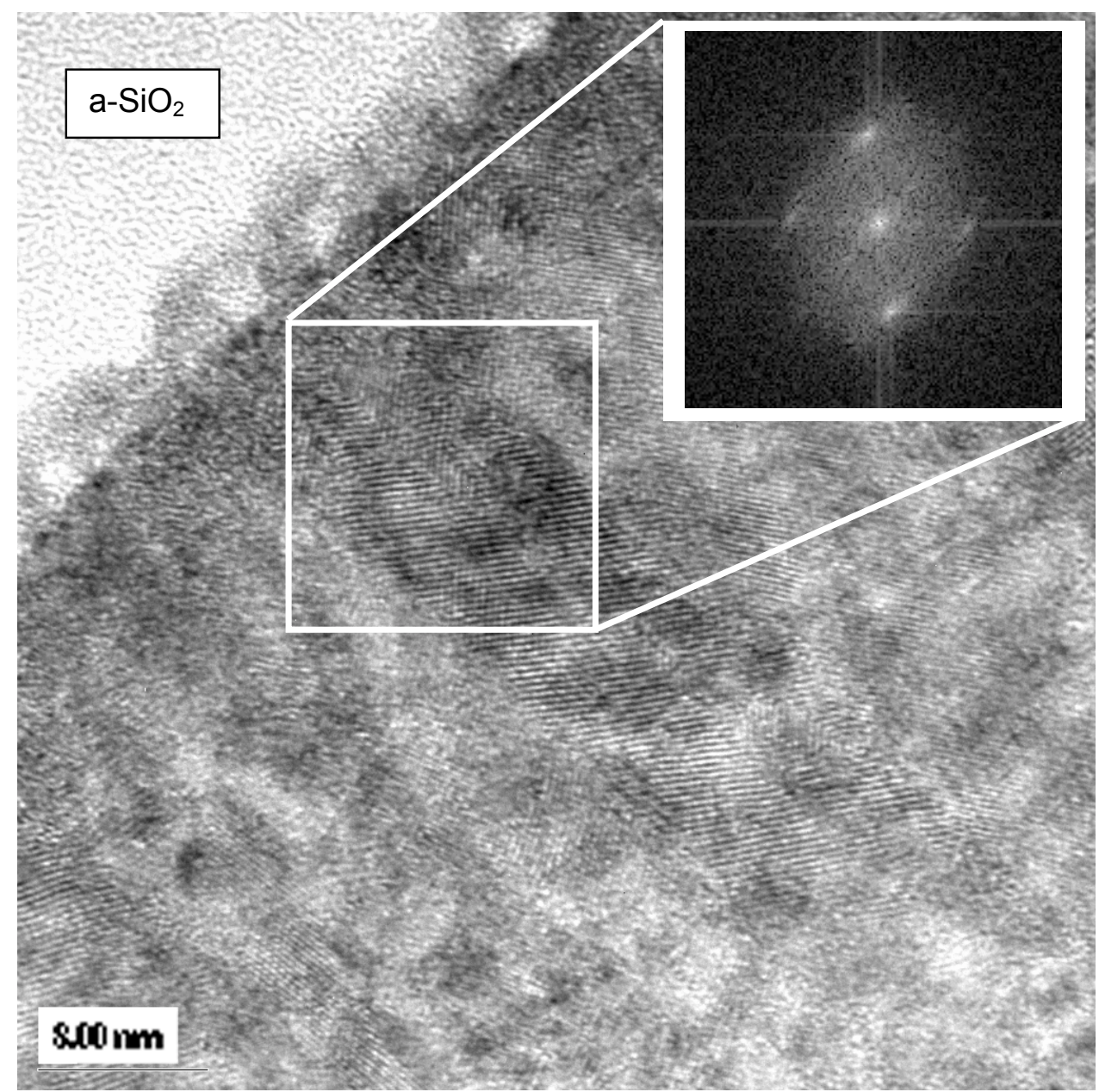

Abb. 4.20: Hochauflösende TEM-Aufnahme an einer bei $100^{\circ} \mathrm{C}$ gesputterten, stöchiometrischen $\mathrm{IrO}_{2}$ Schicht. Das eingefügte Bild stellt die berechnete zweidimensionale Fouriertransformation des markierten Bereiches dar, auf der (110)-Reflexe zu sehen sind, die mit der Schichtnormalen einen Winkel von $45^{\circ}$ einschließen.

In Schichtnormalenrichtung sind anhand der durchgeführten TEM-Analysen an den stöchiometrischen $\mathrm{IrO}_{2}$ Schichten keine Inhomogenitäten bezüglich der Mikrostruktur und der Dichte festzustellen; dieses Resultat konnte durch die Röntgenreflektometriemessungen und RBSAnalysen bestätigt werden. 


\section{Rekristallisationsverhalten der $\mathrm{IrO}_{\mathrm{x}}$ Schichten}

In diesem Abschnitt soll für die gesputterten $\mathrm{IrO}_{x}$ Schichten durch ex-situ und in-situ Analysen der Schichtspannungen, des Schichtwiderstandes und durch thermische Analysen das Relaxations- und Rekristallisationsverhalten untersucht werden. Überdies werden zur mikrostrukturellen und morphologischen Charakterisierung Analysen mittels XRD, STM und REM präsentiert.

\subsection{Rasterelektronenmikroskopische Analysen}

Der Einfluss der Stöchiometrie der $\mathrm{IrO}_{x}$ Proben auf die Qualität und die thermische Stabilität wurde mittels rasterelektronen- und rasterkraftmikroskopischen Analysen untersucht. Mit beiden Methoden zeigt sich übereinstimmend, dass nicht stöchiometrisch deponierte $\operatorname{IrO}_{\mathrm{X}}$ Proben eine signifikante Degradation bei thermischer Nachbehandlung aufweisen.
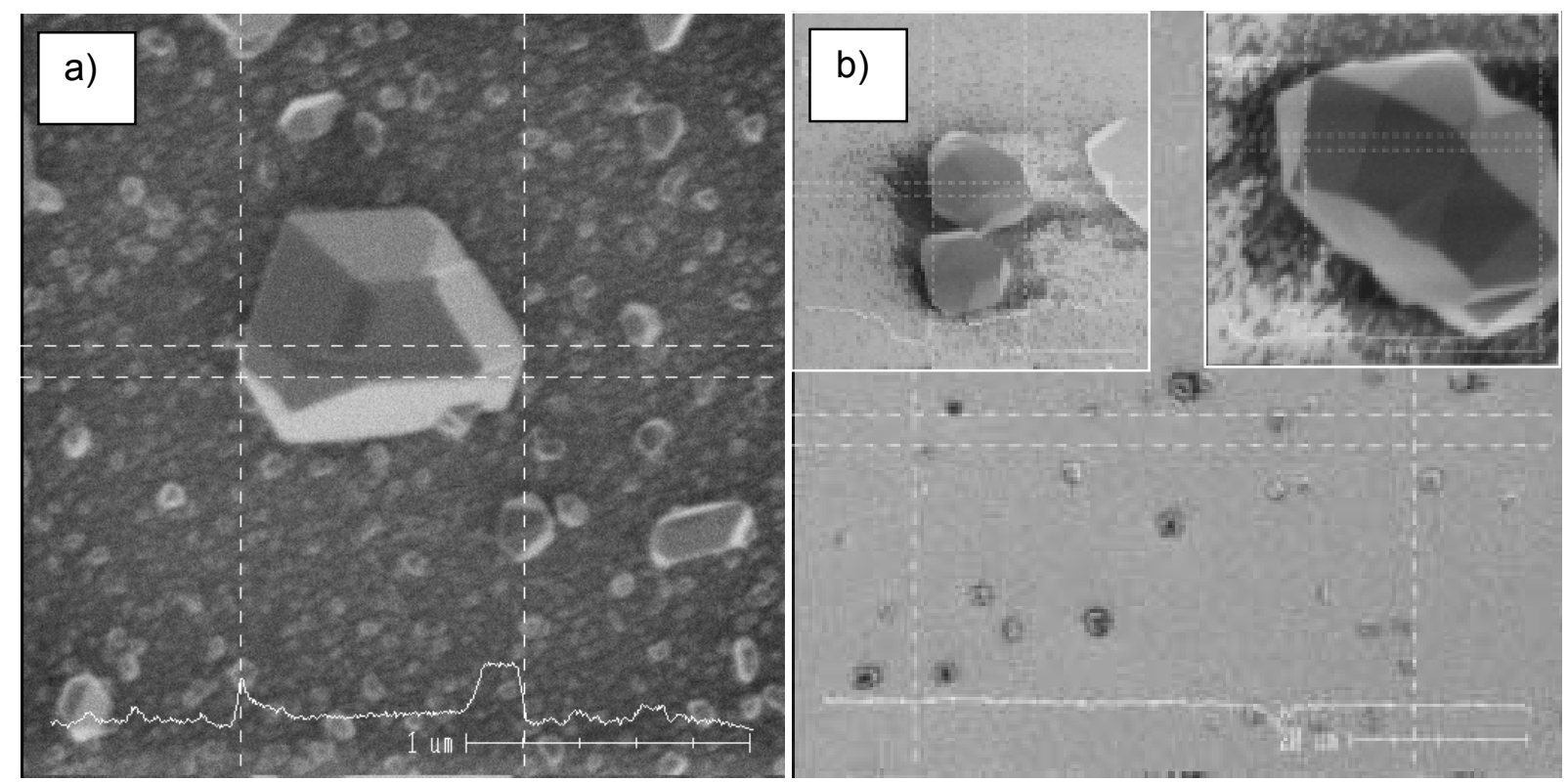

Abb. 5.1: Degradation einer a) sauerstoffunterstöchiometrischen und b) einer sauerstoffüberstöchiometrischen $\mathrm{IrO}_{x}$ Schicht nach einer Temperaturbehandlung von $700^{\circ} \mathrm{C}, 30$ Minuten in Sauerstoffatmosphäre. Die kleinen Bilder in b) zeigen gekippte REM Aufnahmen mit höherer Vergrößerung, sie haben eine laterale Abmessung von 6,5 $\mu \mathrm{m}$ bzw. $2,5 \mu \mathrm{m}$.

Bei geringer Abweichung von der Dioxid-Stöchiometrie beobachtet man eine Aufrauung, nach Auslagerungen bei höheren Temperaturen $\left(T \geq 600^{\circ} \mathrm{C}\right)$ und starken Abweichungen von der Dioxid-Stöchiometrie ein unkontrolliertes Kornwachstum. Die in Abb. 5.1 a) gezeigte REM Analyse der stark sauerstoffunterstöchiometrischen Schicht $(0: \operatorname{Ir} \approx 1: 1)$ zeigt, dass nach der Temperaturbehandlung bei $700^{\circ} \mathrm{C}$ Kristalle auf der Schichtoberfläche entstanden sind, die teilweise auf eine Größe von etwa $1 \mu \mathrm{m}$ angewachsen sind. Diese Kristalle werden durch Auger-Analysen als oberflächlich oxidierte Ir-Kristalle identifiziert. Anhand Abb. 5.1 b) 
lässt sich erkennen, dass schon für leicht sauerstoffüberstöchiometrische Schichten $(O: I r \approx 2,1: 1)$ ein Kollabieren der Schichtintegrität beim Anlassen stattfinden kann. Die auf dem Substrat gewachsenen, teilweise mehrere $\mu \mathrm{m}$ großen Mikrokristalle sind mittels AES als $\mathrm{IrO}_{2}$ Kristalle identifiziert worden, die sich aus der sie umgebenden deutlich porösen Schicht gebildet haben. Vergleichbare Ergebnisse sind auch von Cho et al. [Cho97] in nicht stöchiometrisch deponierten $\mathrm{IrO}_{\mathrm{x}}$ Schichten gefunden worden.

Diese Ergebnisse machen deutlich, dass eine Sauerstoff-Übersättigung der Ir- und der $\mathrm{IrO}_{2}$ Phase zu einer Ausscheidung des Exzess-Sauerstoffes beim Anlassen führt, die die Schichtintegrität zerstört und die Schichten unbrauchbar macht. Bei stöchiometrisch oder leicht sauerstoff-unterstöchiometrisch deponierten $\mathrm{IrO}_{2}$ Schichten sind allerdings mittels Rasterelektronenmikroskop keine signifikanten Veränderungen der Schichtmorphologie sichtbar, so dass das Rekristallisationsverhalten dieser thermodynamisch stabilen Schichten im folgenden Kapitel mittels Röntgenbeugung untersucht werden soll.

\subsection{In-situ Hochtemperatur Diffraktometrieanalysen (HTXRD)}

Das Rekristallisationsverhalten der stöchiometrischen $\mathrm{IrO}_{2}$ Proben ist mittels in-situ HTXRD in Abhängigkeit der Depositionstemperatur $T_{S}$ analysiert worden. Dabei wurde die Reflexform, die relative Reflexintensität, die Reflexbreite und die Reflexlage der beobachteten (110)- und (200)-Reflexe bestimmt und ausgewertet. Da bei dem Heizprozess von Raumtemperatur auf $850^{\circ} \mathrm{C}$ jedoch eine thermische Ausdehnung der Schicht und auch des aus $\mathrm{Al}_{2} \mathrm{O}_{3}$ bestehenden Probenhalters geschieht, ist die absolute Position der Peaks nur unter gewissen vereinfachenden Annahmen quantitativ auszuwerten.

Abb. 5.2 zeigt die röntgenographischen in-situ Messungen der bei $100^{\circ} \mathrm{C}$ und der bei $500^{\circ} \mathrm{C}$ deponierten $\mathrm{IrO}_{2}$ Schicht. Bei der Analyse wurde von den Proben jeweils vor und nach dem Heizprozess ein Spektrum bei Raumtemperatur (RT) als Referenz gemessen. Anhand der Diffraktometriespektren in Abb. 5.2 ist nachweisbar, dass der Rekristallisationsvorgang abhängig von der Depositionstemperatur ist. Die Spektren der bei $500^{\circ} \mathrm{C}$ deponierten $\mathrm{IrO}_{2}$ Schicht zeigen bis zu Heiztemperaturen von etwa $800^{\circ} \mathrm{C}$ keine signifikanten Veränderungen, wohingegen die Spektren der bei $100^{\circ} \mathrm{C}$ gesputterten Probe schon bei niedrigen Temperaturen nachweisbare Veränderungen der Reflexform und der Reflexpositionen aufweisen. Es wird anhand der Spektren in Abb. 5.2 b) deutlich, dass der (200) Reflex bei Anlasstemperaturen $a b 400^{\circ} \mathrm{C}$ eine stark asymmetrische Form annimmt. Dieser Tatsache wurde bei der

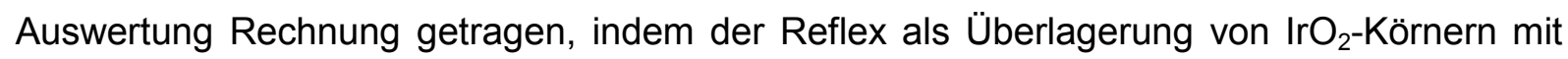
zwei unterschiedlichen Gitterkonstanten angesehen wird. Da die Lage des neu entstehenden (200)-Reflexes nahezu identisch ist mit dem $2 \theta$-Wert einer Referenzpulverprobe [JCP95], wird dieser Reflex einer neu entstehenden Tochtergeneration von $\mathrm{IrO}_{2}$ Körnern mit relaxier- 
ten Netzebenenabständen zugeordnet und deshalb im folgenden mit der Bezeichnung „relaxiert" versehen.
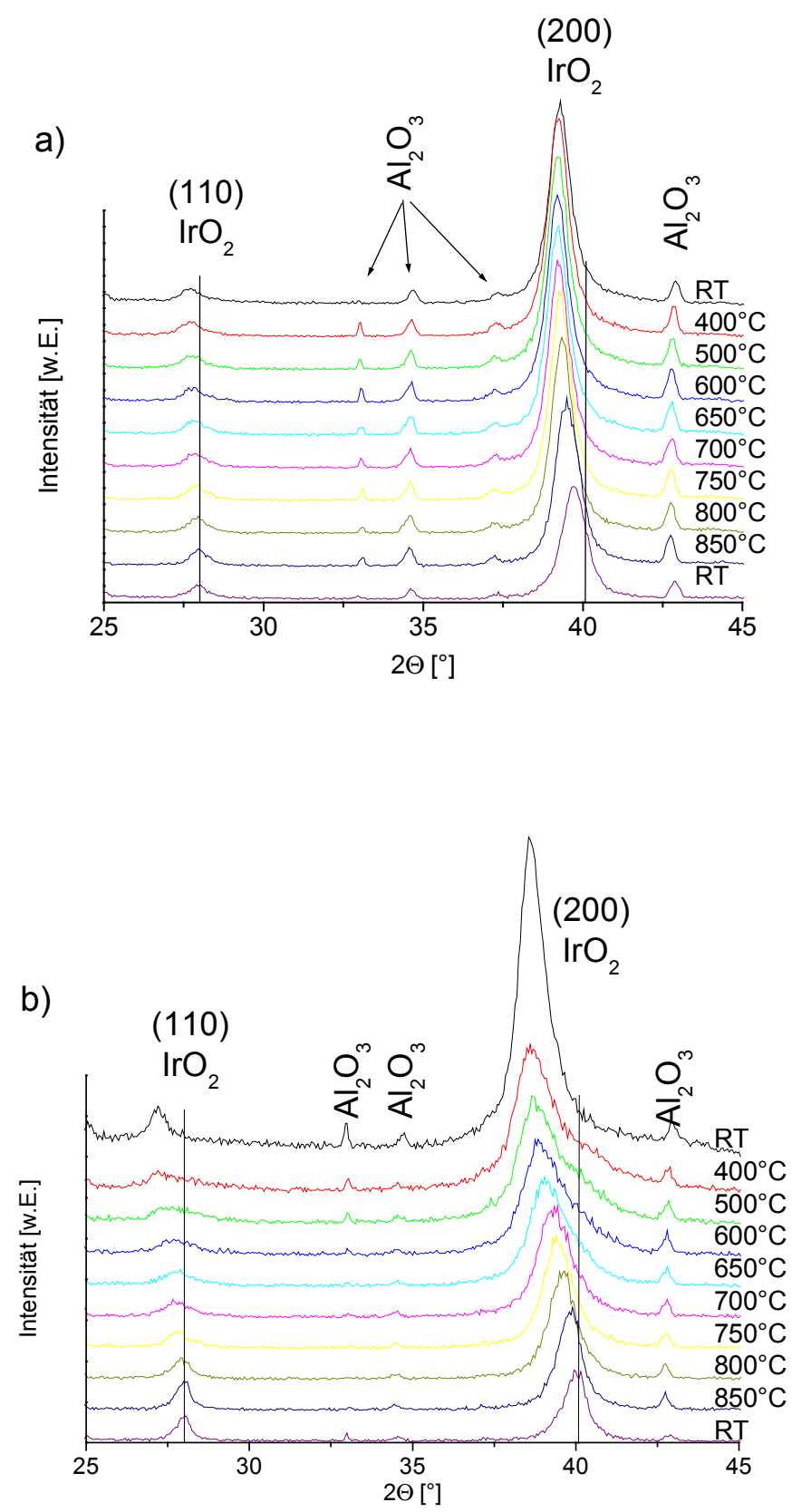

Abb. 5.2: In-situ HTXRD-Analysen der bei $500^{\circ} \mathrm{C}$ (a) und bei $100^{\circ} \mathrm{C}$ (b) gesputterten $\mathrm{IrO}_{2}$ Schichten; die $\mathrm{Al}_{2} \mathrm{O}_{3}$ Reflexe sind durch den Probenträger bedingt. (RT: Raumtemperatur), das jeweils unterste Spektrum ist nach dem Abkühlen der Probe aufgenommen worden.

Das gleiche Phänomen ist für den (110) Reflex ausgeprägt, es zeigt sich eine graduelle Intensitätsabnahme des Reflexes für die Körner mit stark verspannter Gitterkonstante zugunsten des relaxierten Reflexes. In Abb. 5.3 sind die normierten Intensitäten der unrelaxierten 
und der relaxierten Reflexe für die bei $100^{\circ} \mathrm{C}$ gesputterte Probe dargestellt. Anhand der Daten in Abb. 5.3 wird deutlich, dass eine graduelle Ersetzung der unrelaxierten durch relaxierte Körner stattfindet. Dabei scheint dieser Rekristallisationsprozess für die (200)-orientierten Körner höhere Temperaturen zu erfordern, da die Umsetzung erst bei Temperaturen von über $800^{\circ} \mathrm{C}$ vollendet ist, während die Umsetzung für die (110)-orientierten Körner bereits bei $600^{\circ} \mathrm{C}$ abgeschlossen ist. Für die bei $500^{\circ} \mathrm{C}$ gesputterte $\mathrm{IrO}_{2}$ Probe ist ein solches Verhalten nicht zu beobachten, die Reflexposition der dilatierten Körner nähert sich nur bei sehr hohen Anlasstemperaturen $\mathrm{T}>800^{\circ} \mathrm{C}$ langsam dem Referenzwert an, zeigt jedoch auch im wieder abgekühlten Zustand immer noch eine signifikante (200)-Reflexverschiebung, die einer Gitterdilatation von etwa $1 \%$ entspricht.

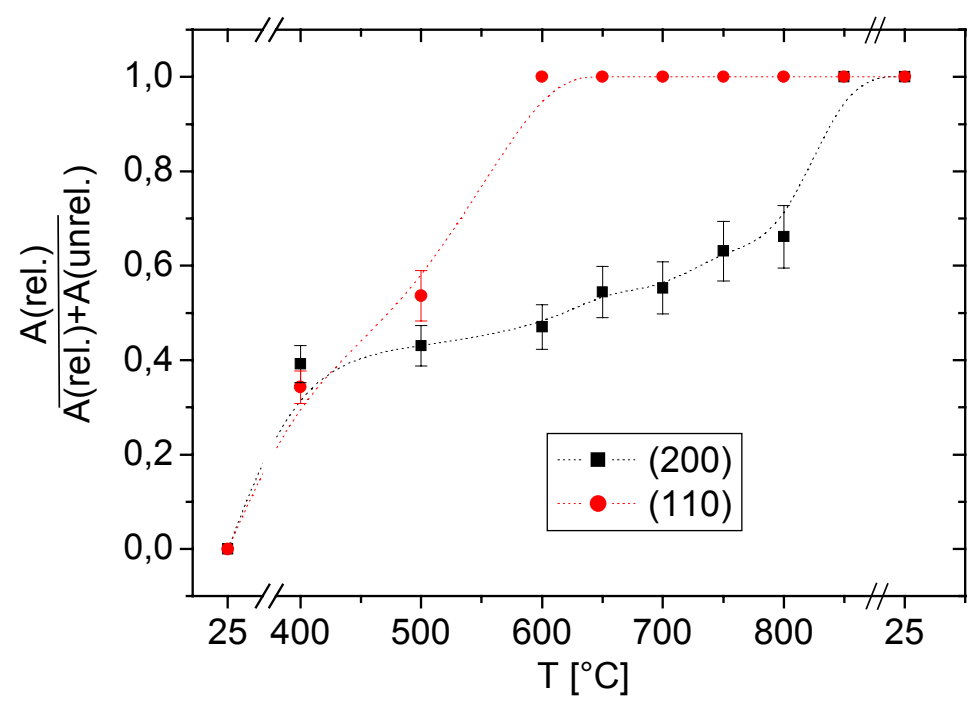

Abb. 5.3: Normierter Anteil der Intensität des jeweils relaxierten Reflexes der bei $T_{S}=100^{\circ} \mathrm{C}$ gesputterten $\mathrm{IrO}_{2}$ Probe während des Anlassens und nach dem Abkühlen. Die Reflexintensität ist die integrale Fläche A der Lorentzanpassung an den Reflex.

Um das Erholungs- und Rekristallisationsverhalten der beiden Proben zu vergleichen, wurde eine Texturauswertung anhand der in Abb. 5.2 gezeigten Spektren durchgeführt. Dazu wurde für jedes Temperatur-Spektrum jeweils das relative Flächenverhältnis $T$ des beobachtbaren (200)-Reflexes zum (110)-Reflex ermittelt und in Abb. 5.4 für die zwei untersuchten Proben aufgetragen. Als integrale Reflex-Intensität wurde dabei sowohl die Fläche des unrelaxierten als auch die des relaxierten Peaks berücksichtigt. Anhand der in Abb. 5.4 gezeigten Texturanalyse ist nachweisbar, dass sich die bei der heißgesputterten Probe $\left(T_{S}=500^{\circ} \mathrm{C}\right) \mathrm{im}$ Ausgangszustand stark ausgeprägte (200)-Textur während des Heizens auf $750^{\circ} \mathrm{C}$ nur geringfügig ändert. Dagegen zeigt die bei $100^{\circ} \mathrm{C}$ deponierte $\mathrm{IrO}_{2}$ Schicht schon bei Anlasstemperaturen über $400^{\circ} \mathrm{C}$ eine deutliche Abnahme der (200)-Textur, die sich nach Durchlaufen des gesamten Heizzyklus auf $\mathrm{T}=4,3: 1$ reduziert hat. 


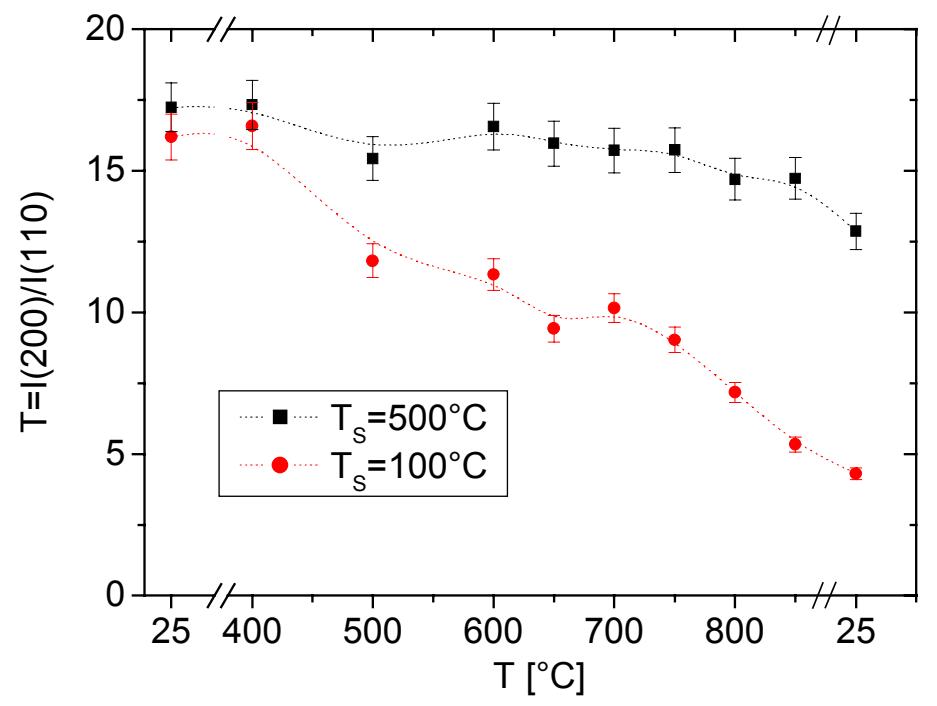

Abb. 5.4: Texturanalyse zur Rekristallisation der $\mathrm{IrO}_{2}$ Schichten für zwei unterschiedliche Depositionstemperaturen $T_{S}=500^{\circ} \mathrm{C}$ und $T_{S}=100^{\circ} \mathrm{C}$ während des Anlassens und nach dem Abkühlen. Dargestellt ist das Flächenverhältnis T des (200)-Reflexes zum (110)-Reflex.

Diese Tatsache spricht für einen früh einsetzenden Rekristallisationsvorgang dieser Probe, bei dem Veränderungen im System der Großwinkelkorngrenzen durch Keimbildung und Wachstum von Körnern mit relaxierten Gitterparametern eine Texturänderung verursachen [Cot67]. Bei der heißgesputterten $\mathrm{IrO}_{2}$ Schicht deuten die bis Anlasstemperaturen von etwa $750^{\circ} \mathrm{C}$ nahezu unveränderte Textur und das anschließende graduelle Relaxieren der Körner auf einen Erholungsmechanismus hin, der erst bei Temperaturen über $800^{\circ} \mathrm{C}$ von einer leichten Texturänderung begleitet wird.

Um die Abhängigkeit des Rekristallisationsvorganges von der Depositionstemperatur mit den Korngrößen zu korrelieren, wurden für beide Proben die Korngrößen mittels Scherrerformel (Gleichung (2.10) abgeschätzt. Die in Abb. 5.5 a) gezeigten Korngrößen für die heißgesputterte Probe lassen erkennen, dass bis zu Temperaturen von $800^{\circ} \mathrm{C}$ kein signifikantes Kornwachstum der (110)-orientierten Körner nachzuweisen ist. Dies ist mit den zuvor gezeigten Ergebnissen konsistent und lässt den Schluss zu, dass bei dieser Probe ausschließlich ein Erholungsvorgang stattfindet, der das Korngrenzengefüge nahezu unverändert lässt. Abb. 5.5 b) zeigt die Korngrößen der bei $100^{\circ} \mathrm{C}$ deponierten Schicht. Dabei wurde auch hier zwischen den Korngrößen der relaxierten und nicht relaxierten Körner unterschieden. Die Keimbildung von extrem feinkörnigen, relaxierten Körnern $(\mathrm{d}=3-7 \mathrm{~nm})$ ist bereits bei einer Anlasstemperatur von $400^{\circ} \mathrm{C}$ nachweisbar. 
a)

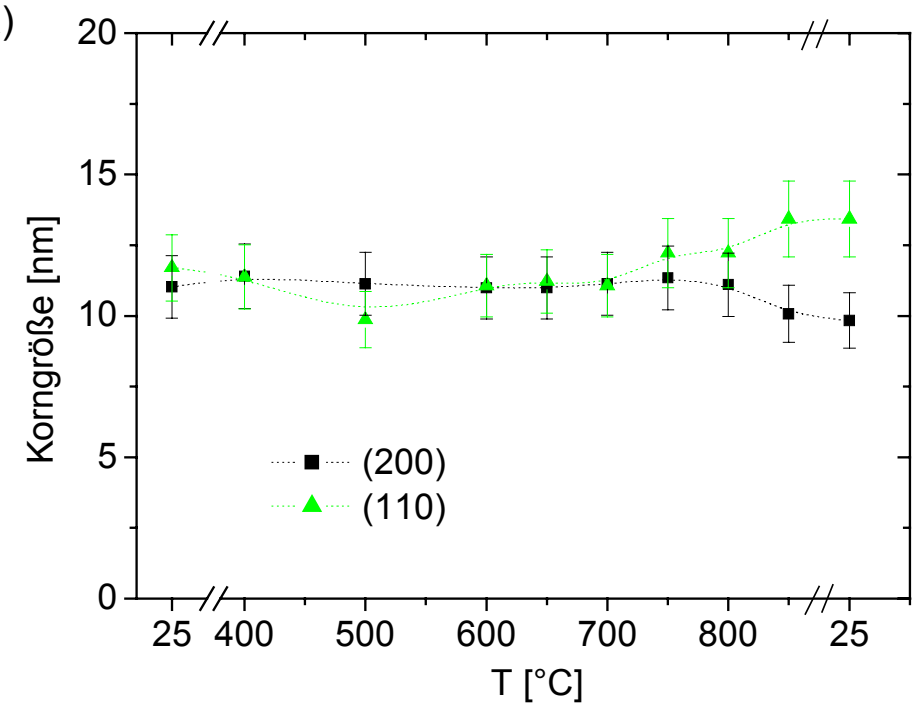

b)

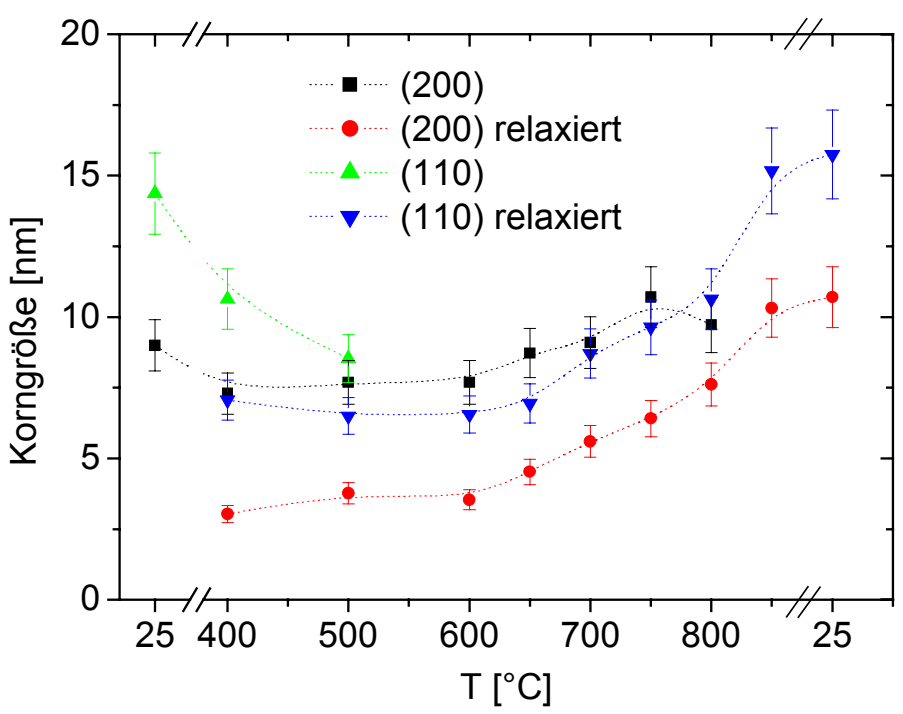

Abb. 5.5: Korngrößen der (a) heiß- und (b) kaltgesputterten $\mathrm{IrO}_{2}$ Schicht während des Anlassvorganges, die mittels Scherrerformel aus dem (110)- bzw. (200)-Reflex bestimmt wurden. Die Verbindungslinien dienen zur Führung des Auges.

Diese neugebildeten Körner wachsen mit zunehmender Temperatur auf eine Größe von etwa $d=10 \mathrm{~nm}$ bzw. $d=15 \mathrm{~nm}$. Dabei ist aus den Daten zu entnehmen, dass die verzerrten, (110)-orientierten Körner bei Temperaturen $\mathrm{T}>500^{\circ} \mathrm{C}$ vollständig ersetzt worden sind, während dilatierte (200)-orientierte Körner mit einer Größe von etwa d=10 nm bis zu Temperaturen von $800^{\circ} \mathrm{C}$ in der Schicht nachgewiesen werden können. 


\subsection{Analyse des elektrischen Widerstands nach kumulativen Temperaturbe- handlungen}

An den gesputterten $\mathrm{IrO}_{2}$ Schichten wurden Analysen zur Veränderung des elektrischen Widerstands nach kumulativer Temperaturbehandlung in Sauerstoff durchgeführt. Dabei wurden die Schichten nacheinander bei $500^{\circ} \mathrm{C}, 600^{\circ} \mathrm{C}$ und $700^{\circ} \mathrm{C}$ für jeweils 30 Minuten in Sauerstoff thermisch nachbehandelt und nach jeder der Behandlungen jeweils der Widerstand bestimmt. Die Ergebnisse sind in Abb. 5.6 zusammengestellt.

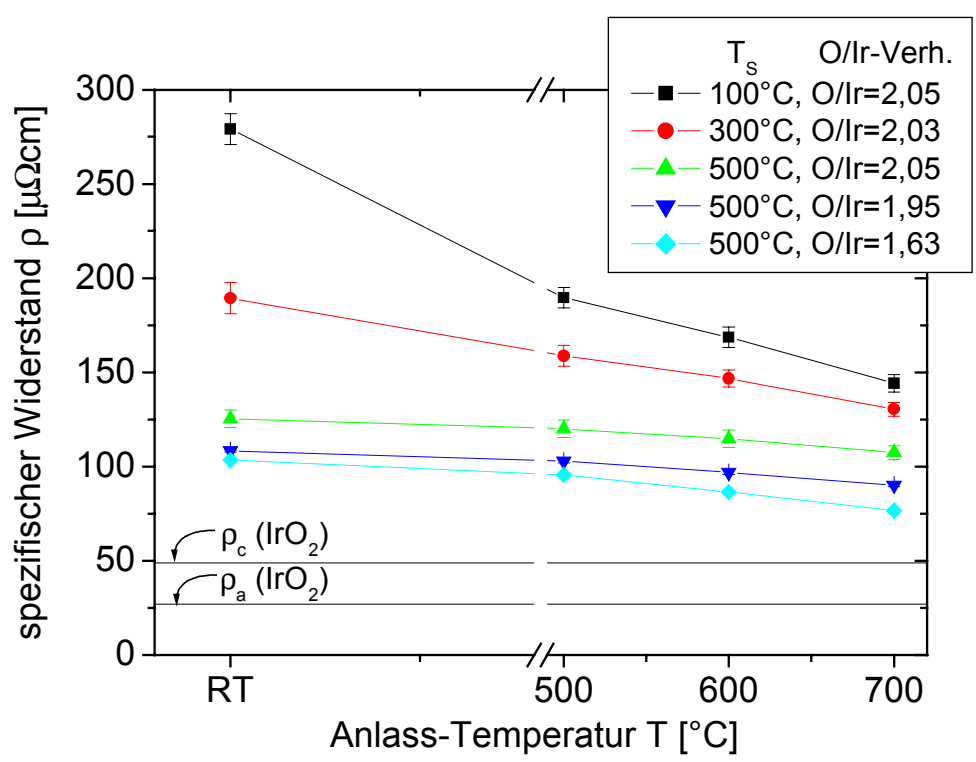

Abb. 5.6: Spezifischer Schichtwiderstand nach kumulativer thermischer Behandlung, (RT: Raumtemperatur, $\rho_{\mathrm{a}}, \rho_{\mathrm{c}}$ : spezifischer Widerstand, der an Einkristallen entlang der a- bzw. c-Achse gemessen worden ist [Gme78]). Die Schichtdicke betrug $d\left(\mathrm{IrO}_{2}\right)=100 \mathrm{~nm}$

Anhand der gezeigten Daten wird deutlich, dass der spezifische Schichtwiderstand der $\mathrm{IrO}_{2}$ Schichten im Ausgangszustand eine unter sonst konstanten Depositionsbedingungen deutliche Abhängigkeit von der Depositionstemperatur aufweist. Dieser Zusammenhang ist bereits in Kap. 4.5 diskutiert worden. Zusätzlich ist in der Abb. 5.6 das elektrische Verhalten zweier Schichten analysiert worden, an denen trotz des Sauerstoffdefizits nach den thermischen Behandlungen keine Degradation nachweisbar war. Diese Schichten sind mit einem Sauerstoffpartialdruck gesputtert worden, bei dem die maximale Korngröße der $\mathrm{IrO}_{2}$ Phase bei einer Abscheidetemperatur von $500^{\circ} \mathrm{C}$ beobachtet werden konnte. Demgemäß ist auch der erniedrigte spezifische Widerstand als eine Erhöhung der freien Weglänge der Ladungsträger in der Schicht interpretierbar. Da jedoch bei den mit diesem Sauerstoffpartialdruck hergestellten Schichten mittels RBS ein signifikantes Sauerstoffdefizit gemessen wurde, scheint der Einfluss der Korngröße auf den elektrischen Widerstand die erhöhte Streuung an zusätzlich vorhandenen Sauerstoffleerstellen überzukompensieren. Liao et al. [Lia98] haben an 
reaktiv gesputterten $\mathrm{IrO}_{2}$ Schichten Untersuchungen zu den elektrischen Eigenschaften angestellt und fanden, dass sich der elektrische Widerstand der Schichten mit einem von Mayadas und Shatzkes [May70] entwickelten Modell, das die Ladungsträgerstreuung an Korngrenzen berücksichtigt, gut beschreiben lässt. Anhand des von Liao et al. [Lia98] gefundenen hohen Streukoeffizienten $\mathrm{S}=0,812$ wird gefolgert, dass für polykristalline $\mathrm{IrO}_{2}$ Schichten die Korngröße der den Schichtwiderstand dominierende Faktor ist. Dieses Ergebnis ist mit den hier gefundenen Daten für den elektrischen Widerstand konsistent, jedoch ist anhand der in dieser Arbeit gefundenen, sehr geringen Korngrößen keine Anpassung an ein solches Modell sinnvoll durchzuführen.

Nach thermischer Behandlung ist für alle Schichten eine graduelle Abnahme des spezifischen Widerstandes zu beobachten. Dabei ist die relative Änderung für die bei $100^{\circ} \mathrm{C}$ deponierte Probe mit etwa $50 \%$ am größten, für die bei $500^{\circ} \mathrm{C}$ deponierten Schichten liegen die relativen Änderungen nur im Bereich von etwa 10-20\%. Unter Berücksichtigung des Modells zur Korngrenzenstreuung ist ein signifikantes Kornwachstum für die bei $500^{\circ} \mathrm{C}$ deponierten Proben deshalb nicht zu erwarten. Diese Folgerung steht in Einklang mit den HTXRD Daten, die ebenfalls nur ein Ausheilen der Defekte aufgrund der Relaxation der Reflexlagen, für Anlasstemperaturen bis $700^{\circ} \mathrm{C}$ jedoch kein Kornwachstum zeigen. Die in Abb. 5.6 gezeigten elektrischen Daten der bei niedrigeren Temperaturen, besonders bei einer Depositionstemperatur von $100^{\circ} \mathrm{C}$ gesputterten $\mathrm{IrO}_{2}$ Schichten legen jedoch die Vermutung nahe, dass aufgrund der deutlichen Reduktion des spezifischen Widerstands auch ein Kornwachstum stattgefunden hat.

Es zeigt sich jedoch, dass nach dem Anlassen bei $700^{\circ} \mathrm{C}$ der Schichtwiderstand der bei $500^{\circ} \mathrm{C}$ gesputterten, stöchiometrischen Probe deutlich unter dem Wert der bei $100^{\circ} \mathrm{C}$ deponierten Probe liegt. Dieses Ergebnis kann so interpretiert werden, dass sich bei der kaltgesputterten Probe $\left(\mathrm{T}_{S}=100^{\circ} \mathrm{C}\right)$ aufgrund des beobachteten Keimbildungs- und Wachstumsprozesses um eine breite Korngrößenverteilung handelt, bei der immer noch ein großer Anteil kleinerer Körner in der Probe vorhanden ist.

\subsection{In-situ Analyse der Schichtspannungen}

Die in-situ Relaxation der Schichtspannungen während des Heizens ist für drei stöchiometrisch abgeschiedene $\mathrm{IrO}_{2}$ Schichten in Abhängigkeit der Abscheidetemperatur $\mathrm{T}_{\mathrm{S}}$ untersucht worden. Um den Einfluss des Si-Substrats auf die Messung abzuschätzen, wurde eine Leermessung mit einem unbeschichteten Si-Substrat durchgeführt. Die maximal beobachtete Abweichung während des gesamten Heizzyklus betrug $\Delta \sigma=0,02 G P a$. Der Spannungsverläufe $\sigma(T)$ für die drei untersuchten $\mathrm{IrO}_{2}$ Proben sind in Abb. 5.7 dargestellt. 


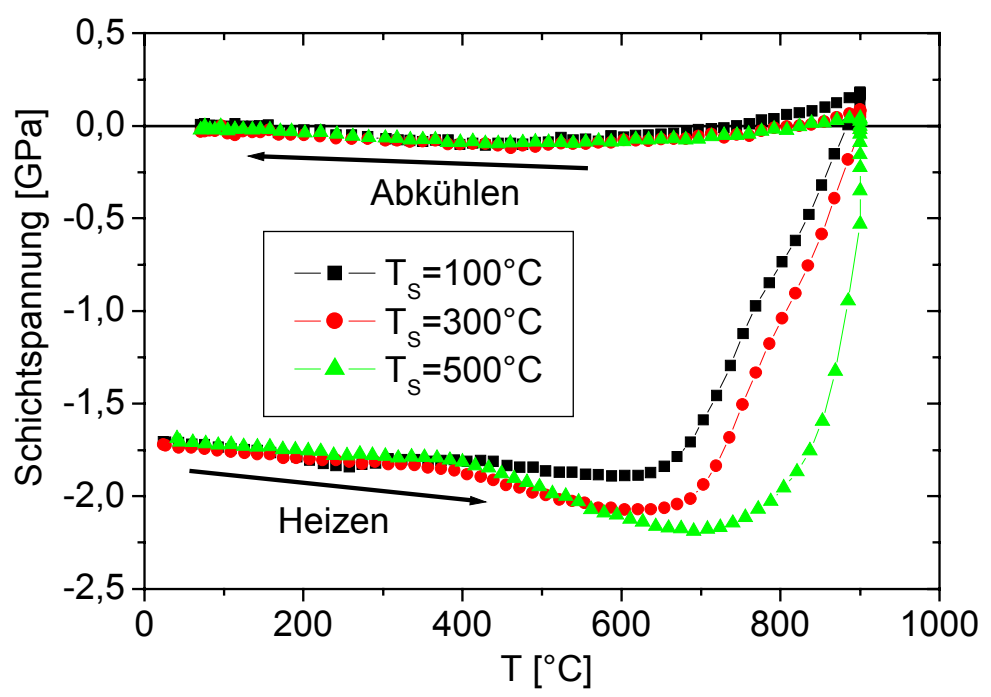

Abb. 5.7: In-situ Spannungsrelaxationsanalyse von $\mathrm{IrO}_{2}$ Schichten in Abhängigkeit der gewählten Depositionstemperatur. Die Schichtdicke beträgt $\mathrm{d}\left(\mathrm{IrO}_{2}\right)=100 \mathrm{~nm}$, als Substrate wurden beidseitig oxidierte Si-Wafer verwendet. Die Proben wurden mit einer Rate von $r=10 \mathrm{~K} / \mathrm{min}$ geheizt.

Wie bereits in Kap. 4.5 diskutiert, zeigt die absolute Spannung der stöchiometrisch gesputterten $\mathrm{IrO}_{2}$ Schichten nach der Abscheidung keine signifikante Temperaturabhängigkeit, was durch eine sehr geringe differentielle thermische Ausdehnung der $1 \mathrm{rO}_{2}$ Schicht zum Si(100) Substrat gedeutet worden ist. Die Annahme lässt sich anhand der Abkühlkurven verifizieren, da die in diesem Bereich entstehenden Spannungen ausschließlich auf die differentielle thermische Ausdehnung zurückzuführen sein sollte, und für alle Schichten nur eine sehr geringe Spannungsänderung erkennbar ist. Wie in Abb. 5.7 gezeigt ist, beobachtet man für alle drei Proben bis zu einer Temperatur von etwa $400^{\circ} \mathrm{C}$ einen gleichen Verlauf der mechanischen Spannungen, dabei nehmen die kompressiven Spannungen geringfügig zu. Dieses Verhalten wird aufgrund der fast identischen Veränderungen der Spannungsentwicklung $\sigma(T)$ beim Aufheizen und beim Abkühlen ausschließlich als differentielle thermische Ausdehnung gedeutet, die sich in einer thermischen Spannung $\sigma_{\text {th }}$ äußert und nach Gleichung (4.6) beschrieben werden kann. Da der thermische Ausdehnungskoeffizient für das $\mathrm{IrO}_{2}$ größer ist als der des Siliziums [Gme78], führt dies zu einer leichten Zunahme der kompressiven Spannungen. Oberhalb einer Temperatur von etwa $400^{\circ} \mathrm{C}$ beginnt der Spannungsverlauf der bei $100^{\circ} \mathrm{C}$ gesputterten Probe jedoch signifikant von den zwei heißgesputterten Proben abzuweichen. Dieses Verhalten kann anhand der HTXRD-Analysen auf den bei Temperaturen zwischen $400^{\circ}$ und $500^{\circ} \mathrm{C}$ einsetzenden Rekristallisationsvorgang und einen gleichzeitigen Relaxationsvorgang im Spannungszustand dieser Probe gedeutet werden. Ab einer Temperatur von etwa $650^{\circ} \mathrm{C}$ ist bei dieser Probe mit zunehmender Temperatur sehr deutlich eine Abnahme der 
Abnahme der kompressiven Spannungen zu beobachten, die mit dem Wachstum der Körner mit relaxierten Gitterkonstanten auf Kosten der dilatierten Körner korreliert wird. Bei Temperaturen von über $850^{\circ} \mathrm{C}$ ist die Probe in einem nahezu spannungsfreien Zustand.

Die heißgesputterten Proben zeigen hingegen oberhalb von $400^{\circ} \mathrm{C}$ eine weitere Zunahme der kompressiven Spannungen, die einer Überlagerung der thermischen Ausdehnung des $\mathrm{IrO}_{2}$ und einem in diesem Temperaturbereich stattfindenden Ausheilprozess zugeschrieben wird. Hier nicht gezeigte in-situ Spannungsmessungen an heißgesputterten $\mathrm{IrO}_{2}$ Proben, die nur bis zu einer maximalen Heiz-Temperatur von $600^{\circ} \mathrm{C}$ durchgeführt worden sind, zeigen, dass es sich bei dieser zusätzlichen Spannungszunahme um einen nahezu reversiblen Effekt handelt, da sich der Gesamtspannungszustand der Probe nach dem Abkühlen im Vergleich zum Ausgangszustand nicht signifikant verändert hat. Eine deutliche, irreversible Spannungsrelaxation findet jedoch erst bei Temperaturen von über $700^{\circ} \mathrm{C}$ für die bei $\mathrm{T}_{\mathrm{S}}=300^{\circ} \mathrm{C}$, bzw. $800^{\circ} \mathrm{C}$ für die bei $\mathrm{T}_{\mathrm{S}}=500^{\circ} \mathrm{C}$ gesputterte Probe statt. Diese Spannungsreduktion kann mit der Erholung und einem anschließenden Kornwachstum erklärt werden [Des99], das mit den HTXRD Analysen bei den genannten Temperaturen für diese Proben nachgewiesen werden konnte. Im Gegensatz zu identisch gemessenen Ir Schichten sind die Proben nach dem Abkühlen frei von tensilen Spannungen. Dieses Resultat wird auf die sehr ähnliche thermische Ausdehnung der $\mathrm{IrO}_{2}$ Schicht und des Si-Substrats zurückgeführt, während das Ir eine deutlich stärkere thermische Ausdehnung als das Si zeigt [Gme78].

\subsection{Differentielle Thermische Analyse}

Anhand von differentieller thermischer Analyse der gesputterten stöchiometrischen $\mathrm{IrO}_{2}$ Schichten ist das Wärmesignal, das die Proben während des Heizzyklus abgeben, gemessen worden. Abb. 5.8 a) zeigt die Signale der drei analysierten Proben $\left(T_{S}=100^{\circ}, 300^{\circ}\right.$ und $500^{\circ} \mathrm{C}$ ), die durch Differenzbildung der ersten und zweiten durchgeführten Messung errechnet worden sind, um mögliche, apparativ bedingte Untergrundsignale abzutrennen. Für die Diskussion der Messdaten sind die gezeigten Graphen in drei Temperaturbereiche (I, II und III) untergliedert. Im Bereich I, der sich bis zu einer Temperatur von etwa $400^{\circ} \mathrm{C}$ erstreckt, ist für die drei Proben kein signifikantes thermisches Signal erkennbar, das auf eine exotherme Probenveränderung hindeutet. Im anschließenden Bereich II ist ein mit der Temperatur zunehmender exothermer Wärmefluss für die $\mathrm{IrO}_{2}$ Schichten erkennbar. Die Größe des gemessenen Signals, bzw. des integralen Wärmeflusses, nimmt systematisch mit abnehmender Depositionstemperatur zu. Der deutlich größere integrale Wärmefluss der kaltgesputterten Probe $\left(T_{S}=100^{\circ} \mathrm{C}\right)$ wird der Rekristallisationswärme zugeschrieben, die beim Ersetzen der durch den Depositionsprozess stark dilatierten Körner durch relaxierte Körner frei wird und als Bereich der primären Rekristallisation angesehen werden kann. 
a)

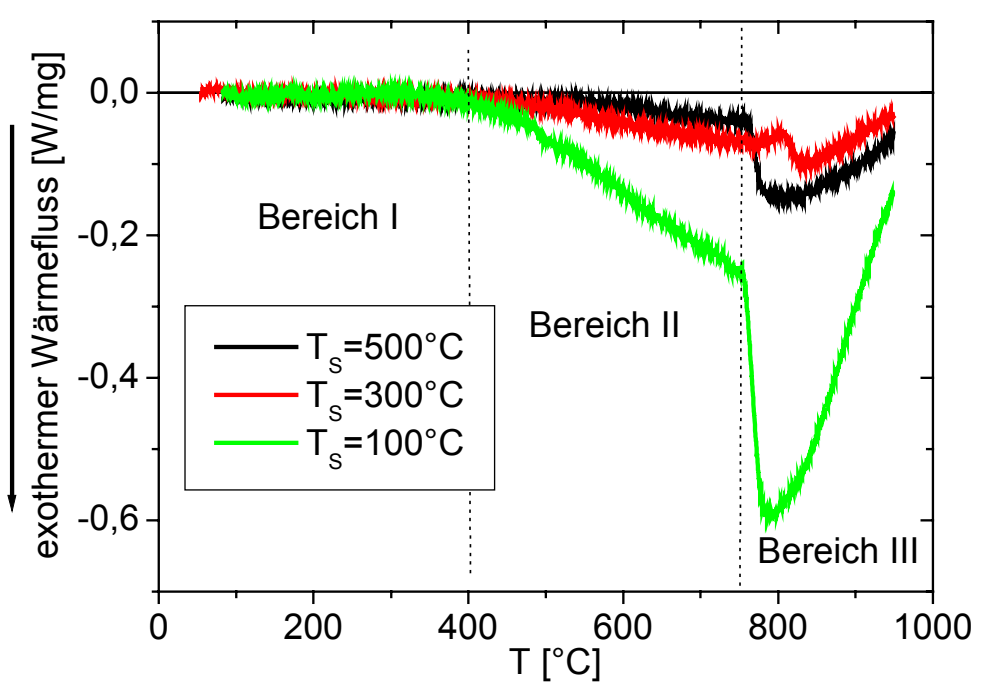

b)

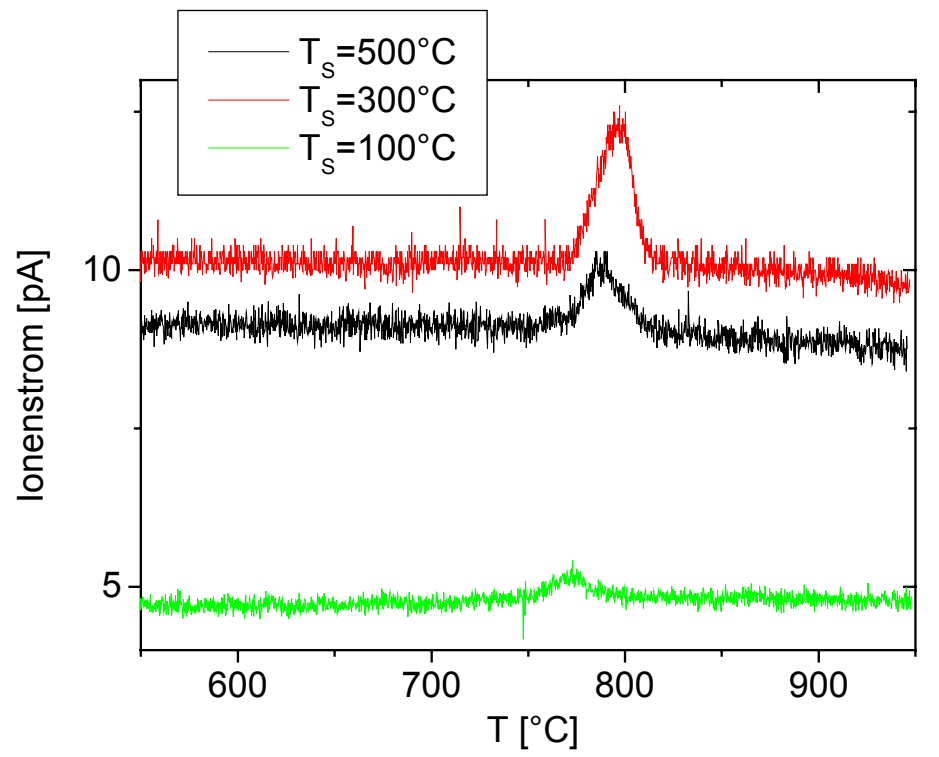

Abb. 5.8: a) Differentielle thermische Signale der $\mathrm{IrO}_{2}$ Schichten während des Heizens, Schichtdicke $\mathrm{d}\left(\mathrm{IrO}_{2}\right)=100 \mathrm{~nm}$, Heizrate $\left.\mathrm{r}=10 \mathrm{~K} / \mathrm{min} \mathrm{b}\right)$ mittels Massenspektrometer detektiertes ${ }^{32} \mathrm{O}_{2}$ Restgassignal

Der in Bereich II für die heißgesputterten Proben auftretende deutlich geringere exotherme Wärmefluss kann aufgrund der HTXRD-Ergebnisse keinem nennenswerten Kornwachstum zuzuordnen sein, sondern ist vielmehr durch das Ausheilen von Gitterdefekten bedingt. Der Übergang von Bereich II in Bereich III ist durch eine kurzzeitige geringe, aber nachweisbare Sauerstoffabgabe der Schichten gekennzeichnet, die mit einer stark exothermen Reaktion verbunden ist. Diese Reaktion wird der Einstellung der korrekten Stöchiometrie durch atomare oder molekulare Abgabe des Exzess-Sauerstoffs zugeschrieben. Dieses Resultat ist konsistent mit den RBS Analysen, die die Ausgangs-Schichten als leicht (1,5-2,5\%) sauerstoff- 
überstöchiometrisch ausgewiesen hatten. Eine kontinuierliche Einstellung eines Sauerstoffdefizits $\mathrm{IrO}_{2-x}$ mit ansteigender Temperatur in der sauerstoffarmen Ar-Atmosphäre gemäß Gmelin [Gme78] kann hier nicht nachgewiesen werden, da das lonenstrommesssignal des Massenspektrometers nach der Sauerstoffabgabe wieder mit dem Untergrund-Messwert vor der Abgabe identisch ist. Thermogravimetrische Messungen zeigen in Bereich III keinen signifikanten Gewichtsverlust der Proben, den man bei einem vermehrten Sauerstoffverlust oder sogar einer Volatilisation von flüchtigem $\mathrm{IrO}_{3}$ erwarten würde. Vielmehr ist der in $\mathrm{Be}-$ reich III nachweisbare exotherme Wärmefluss einem weiteren Abbau von Korngrenzen und einem Kornwachstum zuzuordnen, das mit einer Aufrauung der Proben einhergeht. Dies soll an dieser Stelle jedoch nicht weiter untersucht werden, da Temperaturen von deutlich über $800^{\circ} \mathrm{C}$ für die Integration der Schichten irrelevant sind.

\subsection{STM-Analysen der $\mathrm{IrO}_{2}$ Schichten nach thermischer Behandlung}

Es wurden STM-Analysen an $\mathrm{IrO}_{2}$ Schichten nach kumulativer thermischer Behandlung (vgl. Kap. 5.3 zur elektrischen Charakterisierung) durchgeführt, um den Einfluss der Rekristallisation bzw. des Erholungsprozesses auf die Morphologie und die Rauigkeit der Schichten zu analysieren. Anhand dieser Analysen soll der Einfluss der Depositionstemperatur der $\mathrm{IrO}_{2}$ Schichten untersucht werden. Zu diesem Zweck werden die in Kap. 4.6 vorgestellten STM Daten der Ausgangsschichten als Referenz benutzt.

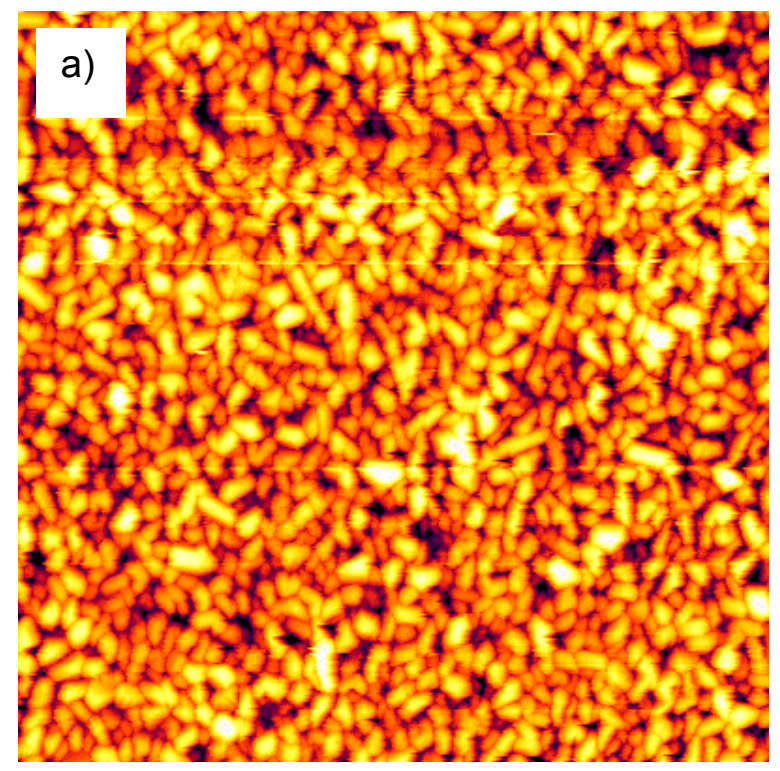

Abb. 5.9: STM-Bild einer $\mathrm{IrO}_{2}$ Schicht $T_{S}=100^{\circ} \mathrm{C}, d=100 \mathrm{~nm}$ auf thermisch oxidiertem Si-Substrat nach $700^{\circ}-\mathrm{O}_{2}$-Auslagerung Kantenlänge $\mid=750 \mathrm{~nm}$ $\square_{\Delta \mathrm{z}=35 \mathrm{~nm}}$ $U=650 \mathrm{mV}, \mathrm{I}=0,8 \mathrm{nA}, \mathrm{f}=1,34 \mathrm{~Hz}$

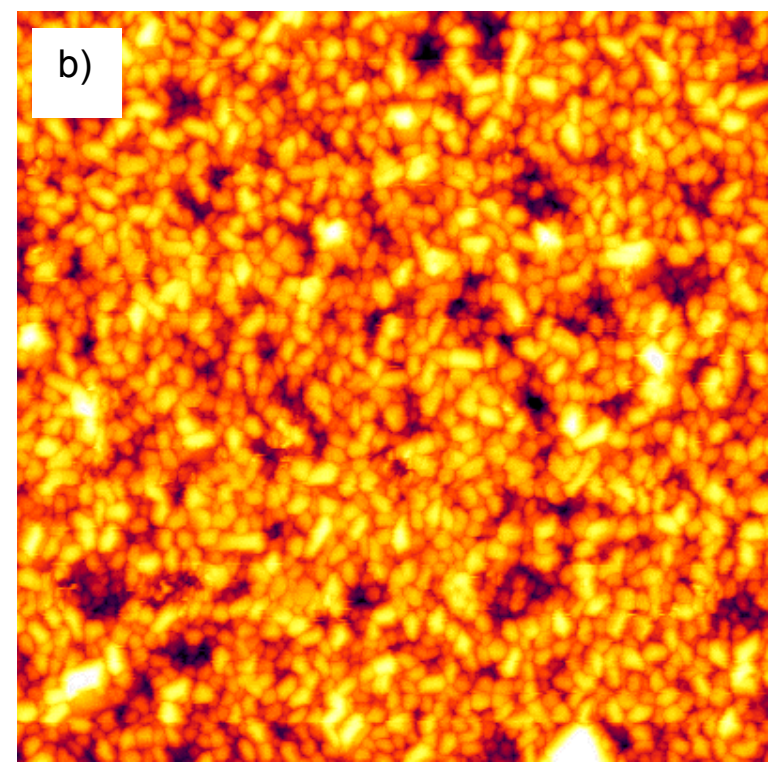

STM-Bild einer $\mathrm{IrO}_{2}$ Schicht, $\mathrm{T}_{\mathrm{S}}=500^{\circ} \mathrm{C}, \mathrm{d}=100 \mathrm{~nm}$ auf thermisch oxidiertem Si-Substrat nach $700^{\circ} \mathrm{C}-\mathrm{O}_{2}$-Auslagerung Kantenlänge $\mathrm{I}=750 \mathrm{~nm}$

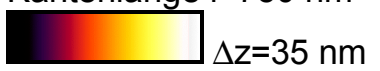
$U=650 \mathrm{mV}, \mathrm{I}=0,8 \mathrm{nA}, \mathrm{f}=1,34 \mathrm{~Hz}$ 
Die in Abb. 5.9 gezeigten STM-Bilder sind von zwei stöchiometrischen IrO $_{2}$ Proben nach der thermischen Behandlung bei $700^{\circ} \mathrm{C}$ aufgenommen worden. Dabei stellt die Abb. 5.9 a) die bei $100^{\circ} \mathrm{C}$ gesputterte Probe dar, die Abb. 5.9 b) die bei $500^{\circ} \mathrm{C}$ hergestellte Probe. Das STM-Bild der bei $100^{\circ} \mathrm{C}$ deponierten Schicht zeigt eine im Vergleich zum Ausgangszustand deutlich veränderte Oberflächenmorphologie. Die im Ausgangszustand rund erscheinenden Strukturen, die mit einer geringen Schichtrauigkeit einhergehen, wirken nach dem Anlassen nicht mehr isotrop, sondern sind den Strukturen der bei $500^{\circ} \mathrm{C}$ deponierten Probe mit der körnigen, nadelförmigen Obeflächenmorphologie ähnlich geworden. Zudem ist anhand der deutlich erweiterten Höhenskala auch eine signifikante Aufrauung nach dem Anlassen der bei $100^{\circ} \mathrm{C}$ gesputterten Schicht bemerkbar. Diese Anzeichen deuten einheitlich darauf hin, dass eine Rekristallisation in der bei $100^{\circ} \mathrm{C}$ deponierten Probe stattgefunden haben muss. Im Gegensatz dazu ist bei der in Abb. 5.9 b) gezeigten heißgesputterten $\mathrm{IrO}_{2}$ Schicht $\left(T_{S}=500^{\circ} \mathrm{C}\right)$ keine signifikante Veränderung zum Ausgangszustand erkennbar. Die Oberflächenmorphologie, die Rauigkeit und die Korngrößen haben sich nicht signifikant oder nur gering verändert. Die Daten der bei $700^{\circ} \mathrm{C}$ angelassenen Proben sind im Vergleich zu den Ausgangsschichten („Nach Abscheidung“) in Tab. 5.1 zusammengefasst.

Tab. 5.1: Vergleich der STM-Daten nach dem Abscheiden und nach dem Anlassen der $\mathrm{IrO}_{2}$ Schichten

\begin{tabular}{|c|c|c|c|c|}
\hline & \multicolumn{2}{|c|}{$\mathrm{IrO}_{2}$ Schicht, $\mathrm{T}_{\mathrm{S}}=100^{\circ} \mathrm{C}$} & \multicolumn{2}{|c|}{$\mathrm{IrO}_{2}$ Schicht, $\mathrm{T}_{\mathrm{S}}=500^{\circ} \mathrm{C}$} \\
\hline & Nach Abscheidung & $\begin{array}{l}\text { Nach } 700^{\circ} \mathrm{C}, \mathrm{O}_{2} \\
30 \text { min. }\end{array}$ & Nach Abscheidung & $\begin{array}{l}\text { Nach } 700^{\circ} \mathrm{C}, \mathrm{O}_{2}, \\
30 \text { min. }\end{array}$ \\
\hline $\begin{array}{l}\text { Rauigkeit } \\
{[\mathrm{nm}]}\end{array}$ & $1,4 \pm 0,1$ & $3,9 \pm 0,3$ & $2,5 \pm 0,2$ & $3,2 \pm 0,5$ \\
\hline $\begin{array}{l}\text { Korngröße } \\
{[\mathrm{nm}]}\end{array}$ & $11,3 \pm 3,2$ & $15,9 \pm 1,1$ & $10,3 \pm 2,0$ & $10,4 \pm 0,4$ \\
\hline
\end{tabular}

\subsection{Diskussion}

An den hier untersuchten gesputterten $\mathrm{IrO}_{2}$ Schichten ist eine signifikante Abhängigkeit des Rekristallisationsprozesses von der Abscheidetemperatur $T_{S}$ festgestellt worden. Dabei konnte eine Erhöhung der Rekristallisationstemperatur bei heißgesputterten Schichten $\left(T_{S}=300^{\circ} \mathrm{C}\right.$, bzw. $\left.500^{\circ} \mathrm{C}\right)$ nachgewiesen werden. Diese Erhöhung kann dadurch gedeutet werden, dass der Deposition ein Verformungs- bzw. Defektbildungsprozess durch den hochenergetischen lonenbeschuss der Schicht überlagert ist. Die Projektilenergien übersteigen 
dabei die Wignerenergie der $\mathrm{IrO}_{2}$ Schicht. Dieser Verformungsprozess kann durch eine Defektbildungsrate $\dot{v}=\dot{v}\left(P, p\left(O_{2}\right), p_{\text {ges }}\right)$ charakterisiert werden, die nach den Ergebnissen aus Kap. 4.3 von der Sputterleistung $P$, dem Sauerstoffpartialdruck $p\left(\mathrm{O}_{2}\right)$ und von dem für die Deposition verwendeten Gesamtdruck $p_{\text {ges }}$ abhängig ist. Durch die Wahl der Substrattemperatur $T_{S}$ kann weiterhin der Grad der thermisch aktivierten Prozesse gesteuert werden. Bei der Wahl einer niedrigen Substrattemperatur $T_{1}<T_{\text {rec }}{ }_{26}$ findet eine Kaltverformung der Schicht statt, bei der kaum signifikante Ausheilprozesse thermisch aktiviert sind, so dass es zur Ausbildung einer Mikrostruktur mit einer hohen Defektdichte kommt. Für Metalle kann die Rekristallisationstemperatur durch $T_{\text {rec }} \approx 0,4 . .0,5 \mathrm{~T}_{\mathrm{m}}$ mit Hilfe der Schmelztemperatur $\mathrm{T}_{\mathrm{m}}$ abgeschätzt werden [Hor95], [Haa94]; unter Benutzung der Sublimationstemperatur des $\mathrm{IrO}_{2}$ von $1100^{\circ} \mathrm{C}$ [Gme78] lässt sich aus dieser Abschätzung eine Rekristallisationstemperatur von etwa 300$400^{\circ} \mathrm{C}$ errechnen. Das Einsetzen der Rekristallisation der kaltgesputterten Probe wurde durch die Analyse der mechanischen Spannungen und die thermische Analyse bei einer Temperatur von $400^{\circ} \mathrm{C}$ beobachtet. Durch Anlassen des stark gestörten Gefüges ist aufgrund der in Kap. 4.3 ermittelten starken Gitterdilatation von etwa 3-4\% und der damit verbundenen hohen Defektdichte eine treibende Kraft vorhanden, die eine Rekristallisation des Gefüges bewirkt. Die in der kaltgesputterten $\mathrm{IrO}_{2}$ Schicht gespeicherte Versetzungsenergie, bzw. der Energiegradient an den Korngrenzen bewirkt, dass Körner mit niedriger Defektdichte auf Kosten von Körnern mit einer signifikant höheren Defektdichte wachsen. Dies äußert sich in einer kontinuierlichen Wärmetönung und in einer nachweisbaren Texturänderung der Schichten.

Durch die Wahl einer höheren Substrattemperatur $T_{2}>T_{\text {rec }}$ findet eine Warmverformung statt, bei der die Defektkonzentration der Schicht stark von der Verformungsgeschwindigkeit abhängt [Hor95]. Bei einer für den reaktiven Sputterprozess angenommenen sehr hohen Defektbildungsrate $\dot{v}$ ist es möglich, in eine heißgesputterte Probe eine hohe Defektdichte einzubringen, da die thermisch aktivierte Annihilation der Defekte die rasche Bildung neuer Defekte nicht vollständig kompensieren kann. Die so hergestellten Schichten zeigen eine für die Herstellungstemperatur ungewöhnlich hohe Defektdichte und benötigen für das komplette Ausheilen eine deutlich höhere Anlasstemperatur [Hor95]. Für die heißgesputterten $\mathrm{IrO}_{2}$ Schichten wurde aufgrund des niedrigeren kristallographischen Verformungsgrades bis zu Anlasstemperaturen von $750^{\circ} \mathrm{C}$ kein Rekristallisationsprozess, sondern vielmehr ein Erholungsprozess nachgewiesen. Die Auswirkungen dieser unterschiedlichen Rekristallisationsverhalten auf die Eigenschaft der $\mathrm{IrO}_{2}$ Schichten als Sauerstoffbarriere werden im folgenden Kapitel diskutiert.

\footnotetext{
${ }^{62} \mathrm{~T}_{\text {rec }}$ Rekristallisationstemperatur
} 


\section{Sauerstoffdiffusionsmessungen an $\mathrm{IrO}_{2}$ und Ir Schichten}

\subsection{Verwendeter Diffusionsaufbau}

Zur Untersuchung der ${ }^{18} \mathrm{O}$ Tracer Diffusion in den $\mathrm{IrO}_{2}$ Schichten wurde ein kommerzieller Ofen Typ HTRH 100/600/17 der Firma Gero 3 benutzt. Für die Temperaturmessung in dem untersuchten Temperaturintervall zwischen $550^{\circ} \mathrm{C}$ und $765^{\circ} \mathrm{C}$ diente ein $\mathrm{Pt} / \mathrm{Rh}$ Thermoelement $\frac{64}{4}$, das zusammen mit dem evakuierbaren Quarzrohr $\left(V_{Q}=353 \mathrm{ml}\right)$ in dem Rohrofen positioniert war. Der Vakuumaufbau ist schematisch in Abb. 6.1 gezeigt.

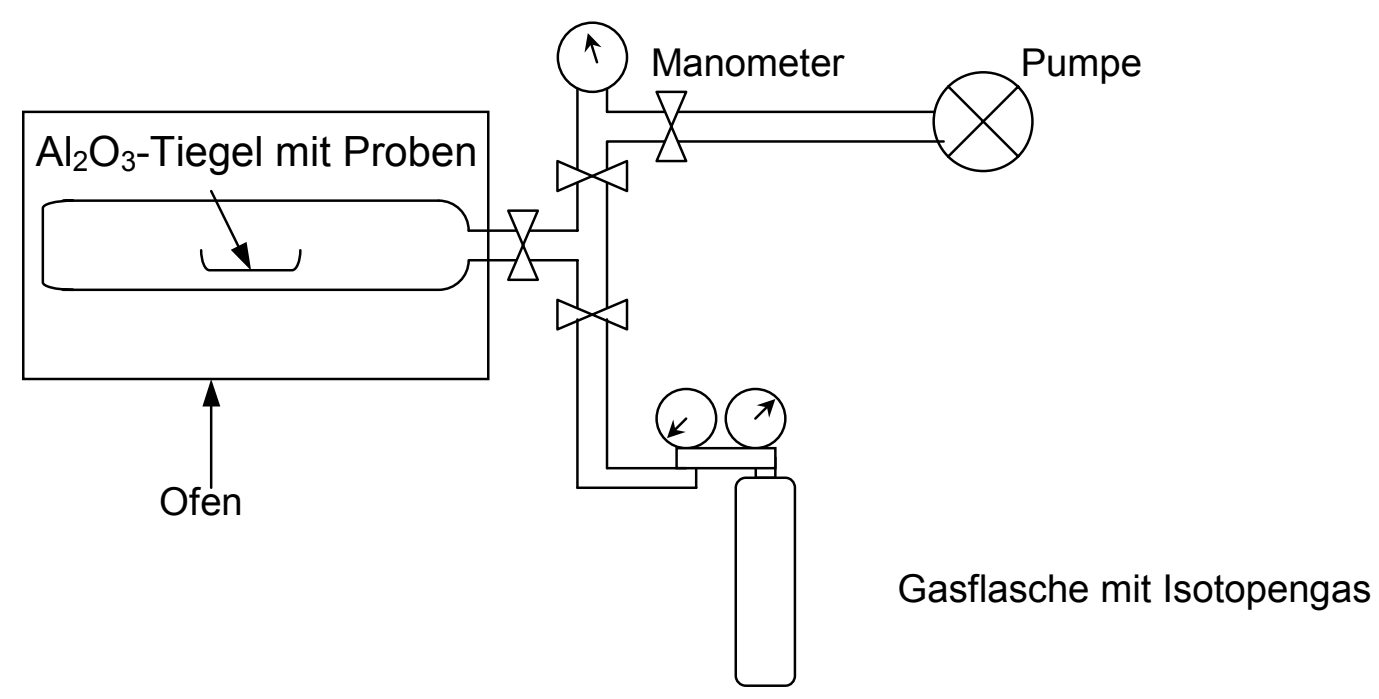

Abb. 6.1: Apparatur zur Diffusionsglühung in ${ }^{18} \mathrm{O}_{2}$ Atmosphäre

Nach Erreichen der Solltemperatur wurde das Quarzgefäß, in dem sich der $\mathrm{Al}_{2} \mathrm{O}_{3}$ Tiegel mit den ca. $10 \mathrm{~mm} \times 10 \mathrm{~mm}$ großen Proben befand, über einen Dreiwegehahn auf ein Grobvakuum evakuiert und anschließend Isotopen-Sauerstoff eingelassen, so dass sich ein Partialdruck von $p\left({ }^{18} \mathrm{O}_{2}\right)=180$ mbar einstellte ${ }^{6.5}$ Nach der Befüllung mit dem Isotopengas wurde das Quarzgefäß über ein Ventil abgeschlossen. Das Ausschleusen der Proben erfolgte nach der Glühung durch Entnahme des Tiegels aus dem Ofen.

\footnotetext{
${ }^{63}$ Gero GmbH, D-75242 Neuhausen, http://www.gero-gmbh.de

${ }^{64} \mathrm{TypB}: \mathrm{Pt}_{30} \mathrm{Rh}_{70} / \mathrm{Pt}_{6} \mathrm{Rh}_{94}$

$6599,5 \%$ angereichertes ${ }^{18} \mathrm{O}_{2}$, Firma Linde AG, D-82049 Höllriegelskreuth, http://e-comgmbh.de/linde-gas/
} 


\subsection{Präparation der $\mathrm{IrO}_{2}$ Proben}

Die $\mathrm{IrO}_{2}$ Proben mit einer Schichtdicke von $\mathrm{d}=300 \mathrm{~nm} \mathrm{~d}=500 \mathrm{~nm}$ wurden wie in Kap. 3.5 beschrieben auf thermische oxidierte Si(100) Wafer gesputtert. Für die Abscheidung wurde eine Depositionstemperatur von $T_{S}=500^{\circ} \mathrm{C}$ und eine Sputterleistung von $P=300 \mathrm{~W}$ gewählt. Dabei wurde der Sauerstoffpartialdruck so eingestellt, dass nahezu stöchiometrische $1 \mathrm{IO}_{2}$ Proben mit einem Texturverhältnis $T=I(110) / I(200) \approx 4$ hergestellt wurden, welches dem Verhältnis einer untexturierten Referenzprobe entspricht. Die so gesputterten Schichten weisen die höchste beobachtete thermische Stabilität bei $800^{\circ} \mathrm{C}$ auf, ohne dass eine Oberflächendegradation auftritt. Nach der Abscheidung wurden die Schichten in reinem ${ }^{16} \mathrm{O}_{2}$ bei Atmosphärendruck für zwei Stunden bei einer Temperatur von $800^{\circ} \mathrm{C}$ geglüht, um für die Diffusionsmessungen im Temperaturbereich von $600^{\circ}<T<765^{\circ} \mathrm{C}$ stationäre Proben mit niedrigen Defektdichten herzustellen. Anhand des in Abb. $\mathbf{6 . 2}$ gezeigten Röntgenweitwinkelbeugungsspektrums konnte die ausschließliche Existenz sehr feinkristalliner Körner $(\mathrm{d}=5-10 \mathrm{~nm})$ der tetragonalen $\mathrm{IrO}_{2}$ Phase nachgewiesen werden. Durch die Analyse der Reflexlagen wurde zudem festgestellt, ob röntgenographisch signifikante Gitterdefekte nachgewiesen werden können. Dazu sind die Ist-Reflexpositionen aus dem in Abb. 6.2 gezeigten Spektrum den Referenzpositionen in Tab. 6.1 gegenüber gestellt.

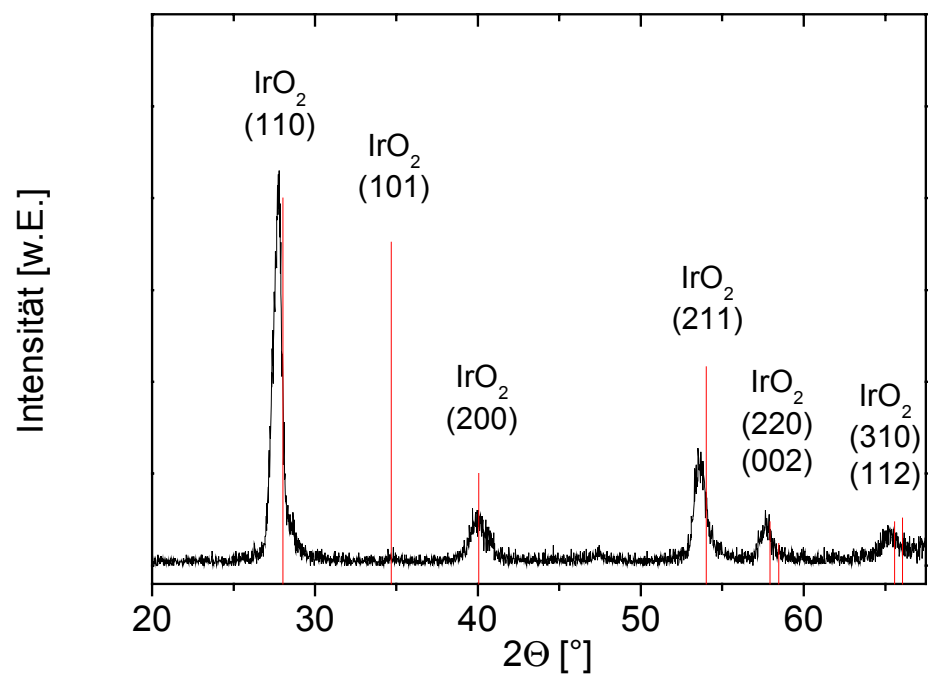

Abb. 6.2: XRD-Analyse der bei $500^{\circ} \mathrm{C}$ und mit $300 \mathrm{~W}$ gesputterten $\mathrm{IrO}_{2}$ Probe nach zweistündiger Glühung bei $800^{\circ} \mathrm{C}$ in Sauerstoff. Als Referenz sind mit roten Linien die Reflexpositionen und die relativen Intensitäten einer untexturierten Pulverprobe angegeben [JCP95].

Mit Gleichung (4.2) errechnet man für ein tetragonales Gitter für den etwas asymmetrischen (110) Reflex eine Gitterdilatation von etwa $0,8 \%$, für alle anderen nachweisbaren Reflexe eine Verzerrung, die deutlich kleiner ist. Die Asymmetrie des (110) Reflexes wird auf die bei 
$800^{\circ} \mathrm{C}$ ablaufende Rekristallisation der Probe zurückgeführt. Aus der noch nicht vollständig abgeschlossenen Relaxation wird gefolgert, dass noch geringe residuale Gitterdefekte in der Probe vorhanden sind.

Tab. 6.1: XRD Reflexpositionen und daraus errechnete Gitterdilatation nach der Temperaturbehandlung bei $800^{\circ} \mathrm{C}, 2 \mathrm{~h}$. Referenzdaten nach [JCP95]

\begin{tabular}{|c|c|c|c|c|c|}
\hline $\begin{array}{l}\mathrm{IrO}_{2} \\
\text { Reflex }\end{array}$ & $\begin{array}{l}\text { Ist Position } \\
2 \theta\left[^{\circ}\right]\end{array}$ & $\begin{array}{l}\text { Referenz Posi- } \\
\text { tion } 2 \theta\left[^{\circ}\right]\end{array}$ & $\begin{array}{l}\text { Relative Git- } \\
\text { terdilatation } \\
\Delta \mathbf{a} / \mathbf{a}[\%]\end{array}$ & $\begin{array}{l}\text { Gemessene } \\
\text { Peak-Intensität } \\
\mathrm{I}(110)=100\end{array}$ & $\begin{array}{l}\text { Referenz Peak- } \\
\text { Intensität } \\
I(110)=100\end{array}$ \\
\hline (110) & 27,75 & 28,027 & 0,80 & 100 & 100 \\
\hline (101) & --- & 34,700 & -- & --- & 88 \\
\hline (200) & 39,94 & 40,058 & 0,27 & 23 & 25 \\
\hline (211) & 53,68 & 54,020 & 0,58 & 38 & 54 \\
\hline (220) & 57,70 & 57,937 & 0,37 & 18 & 12 \\
\hline$(002)$ & --- & 58,469 & -- & --- & 6 \\
\hline (310) & 65,51 & 65,571 & 0,08 & 31 & 12 \\
\hline
\end{tabular}

Bei dem Versuch, vollständig relaxierte $\mathrm{IrO}_{2}$ Schichten zu präparieren, also bei längeren thermischen Vorbehandlungen $(\mathrm{t}>>2 \mathrm{~h})$ und bei höheren Vorbehandlungstemperaturen $\left(T=850-900^{\circ} \mathrm{C}\right)$, wurde an den Proben eine durch das Kornwachstum einsetzende Oberflächenaufrauung und eine durch die Spannungsrelaxation bedingte Rissbildung in den Schichten beobachtet. Die so degradierten Schichten sind jedoch für die SIMS Tiefenprofilanalysen unbrauchbar.

\subsection{Messung und Auswertung der ${ }^{18} \mathrm{O}_{2}$ Isotopenprofile}

Für die zur Auswertung verwendbaren Messpunkte und zur Kalibrierung der relativen Isotopen-Nachweisgenauigkeit ist ein SIMS-Tiefenprofil einer nicht in ${ }^{18} \mathrm{O}$ geglühten Referenzprobe gemessen worden. Diese in Abb. 6.3 präsentierte Messung zeigt das Intensitätsprofil der ${ }^{16} \mathrm{O}^{+}$Ionen und der ${ }^{18} \mathrm{O}^{+}$Ionen. Dabei ist das ${ }^{18} \mathrm{O}$ Profil um den Quotienten der relativen Isotopenhäufigkeiten $\gamma=499$, der direkt aus Tab. 6.2 folgt, hochskaliert worden. Das unter dem $\mathrm{IrO}_{2}$ befindliche $\mathrm{SiO}_{2}$ bewirkt eine deutliche Verringerung der Zählrate um etwa 3 Größenordungen. 


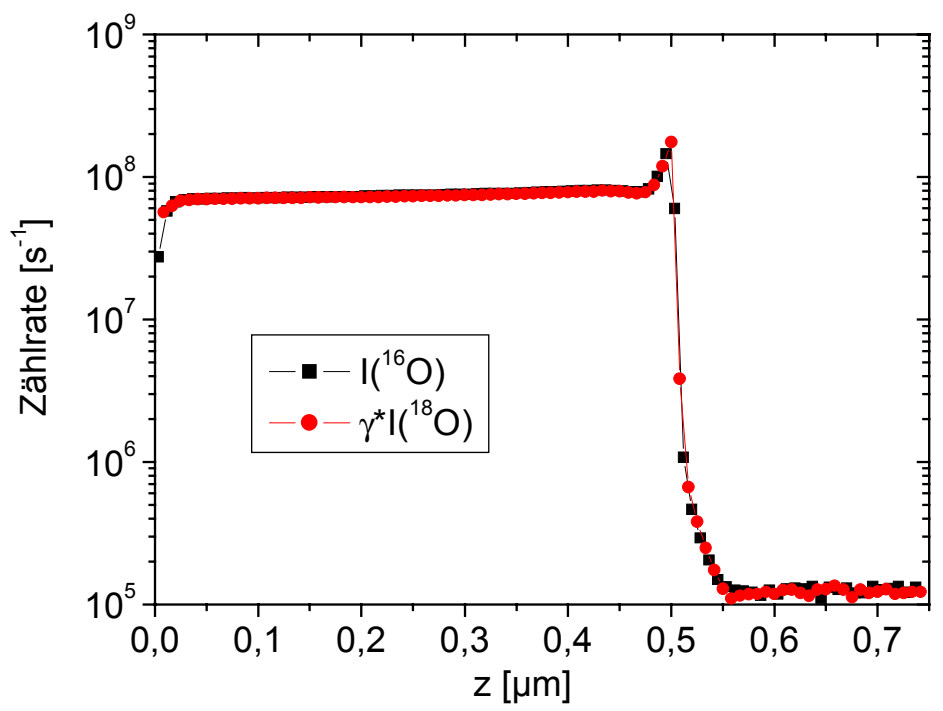

Abb. 6.3: SIMS Intensitätsprofile der ${ }^{16} \mathrm{O}$ und ${ }^{18} \mathrm{O}$ lonen in der nicht in ${ }^{18} \mathrm{O}$ geglühten $\mathrm{IrO}_{2}$ Referenzprobe, $\mathrm{d}\left(\mathrm{IrO}_{2}\right)=500 \mathrm{~nm}$. Das ${ }^{18} \mathrm{O}$ Isotopensignal ist um den Faktor $\gamma$ der relativen Isotopenhäufigkeiten hochskaliert worden.

Es hat sich aus hier nicht erläuterten Messungen herausgestellt, dass für die Trennung von ${ }^{18} \mathrm{O}^{+}$und $\mathrm{H}_{2}^{16} \mathrm{O}^{+}$Ionen mit einer Massentrennung von $M / \Delta M \approx 1900$ gearbeitet werden muss, um die Wasserdampfionen aus dem für die ${ }^{18} \mathrm{O}-$ Konzentrationsbestimmung zu messenden M/e-Signal herauszufiltern. Dies geschieht durch Justieren eines vor dem Detektor befindlichen Austrittsspaltes, der die unerwünschten lonenarten zurückhält. Aus der maximal $2 \%$ abweichenden Übereinstimmung der beiden in Abb. 6.3 gezeigten Signale wird deutlich, dass durch diese Separation tatsächlich nur die ${ }^{18} \mathrm{O}$ lonen nachgewiesen werden.

Tab. 6.2: Isotopendaten zum Sauerstoff nach [web01], [Biè93], [Wal89]

\begin{tabular}{lll}
\hline Isotop & Atomare Masse $\mathbf{M}_{\mathrm{a}}[\mathrm{u}]$ & Natürliches Vorkommen [at\%] \\
\hline${ }^{16} \mathrm{O}$ & $15.99491463(5)$ & 99,762 \\
${ }^{17} \mathrm{O}$ & $16.9991312(4)$ & 0,038 \\
${ }^{18} \mathrm{O}$ & $17.9991603(9)$ & 0,200 \\
\hline
\end{tabular}

Anhand des Tiefenprofils dieser Referenzprobe wird weiter deutlich, dass die Intensität der ersten 2-3 Messpunkte durch typische Einschwingvorgänge des für die Massenseparation benutzten Magneten der CAMECA $6 f$ verfälscht werden, und diese deswegen von der Auswertung ausgeschlossen werden müssen. Die Lage der Grenzfläche $1 \mathrm{IO}_{2} / \mathrm{SiO}_{2}$ ist durch die Intensitätszunahme (sogenanntes Pile-up) und das anschließende starke Abfallen der Signa- 
le zu erkennen. Das Ir-Signal wurde für die Analysen nicht mitgemessen, um eine durch die hohe Massendifferenz zum Sauerstoff bedingte zeitliche Verzögerung zwischen den einzelnen Sauerstoffmesssignalen zu vermeiden und dadurch eine für die Auswertung adäquate Datenpunktdichte zu erzielen. Abb. 6.4 zeigt die SIMS Sauerstoff-Intensitätsprofile der bei $730^{\circ} \mathrm{C}$ geglühten $\mathrm{IrO}_{2}$ Probe. Dabei ist zur besseren Vergleichbarkeit das ${ }^{18} \mathrm{O}$-Signal wieder um den Faktor $\gamma$ hochskaliert worden, um den eindiffundierten Isotopen-Sauerstoff direkt sichtbar zu machen.

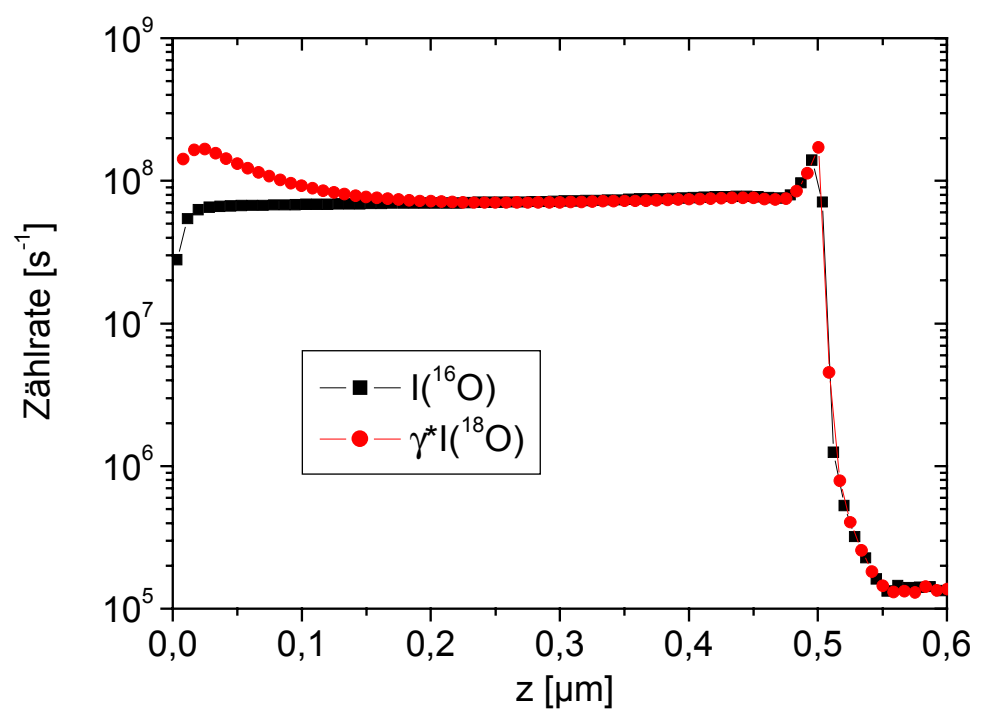

Abb. 6.4: Gemessene SIMS Sauerstoff-Intensitätsprofile, Glühtemperatur $T=730^{\circ} \mathrm{C}$, Glühzeit $\mathrm{t}=20 \mathrm{~h}$. Zur besseren Veranschaulichung wurde das ${ }^{18} \mathrm{O}-M e s s s i g n a l$ um das natürliche Isotopenverhältnis hochskaliert.

Die gemessenen Sauerstoffintensitäten werden gemäß

$f_{m o l}^{18}=\frac{I\left({ }^{18} O\right)}{I\left({ }^{16} O\right)+I\left({ }^{18} O\right)}$

in molare Anteile umgerechnet, die proportional zur wahren ${ }^{18} \mathrm{O}$ Konzentration sind, wobei $f_{m o l}^{18}$ den Molenbruch und $I$ die gemessene SIMS Intensität darstellt [Bro99]. Dabei wird das dritte stabile, natürlich vorkommende Isotop ${ }^{17} \mathrm{O}$ des Sauerstoffs vernachlässigt. Diese Näherung ist gerechtfertigt, da die relative Häufigkeit dieses Isotops etwa vier Größenordnungen unter der des ${ }^{16} \mathrm{O}$ liegt, wie man anhand der Isotopendaten des Sauerstoffs, die in Tab. 6.2 dargestellt sind, erkennen kann. Für die Auswertung der Isotopenkonzentrationsprofile wird die in der Probe natürlich vorhandene ${ }^{18} \mathrm{O}$-Konzentration von der gemessenen Konzentration subtrahiert. Da bei den hergestellten Schichten die Schichtdicke über Simulation von XRR Spektren exakt bekannt ist, wird die mittels profilometrischer Ausmessung gewonnene Tie- 
fenskala der wahren $\mathrm{IrO}_{2}$ Schichtdicke angepasst. Dies ist nötig, da die Sputterrate im darunter liegenden $\mathrm{SiO}_{2}$ nicht mit der des $\mathrm{IrO}_{2}$ übereinstimmt.

Die Lösung der eindimensionalen Differentialgleichung für die Diffusion mit konstantem Diffusionskoeffizienten und konstanter Diffusor-Quelle liefert [Cra67]

$c(z, t)=\frac{c_{0}}{2} \cdot \operatorname{erfc}\left(\frac{z}{2 \sqrt{D^{T} t}}\right)$.

Dabei ist $c_{0}$ die konstante Oberflächenkonzentration des Diffusors, $z$ ist die Tiefenkoordinate, $t$ ist die Glühdauer und $D^{T}$ stellt den als orts- und konzentrationsunabhängig angenommenen Tracer-Diffusionskoeffizienten des Isotopensauerstoffs dar. Mit $\operatorname{erfc}(z)$ wird die komplementäre Fehlerfunktion bezeichnet, die gegeben ist durch [Cra67]

$\operatorname{erfc}(z)=1-\operatorname{erf}(z)=1-\frac{2}{\sqrt{\pi}} \int_{0}^{z} \exp \left(-x^{2}\right) d x$

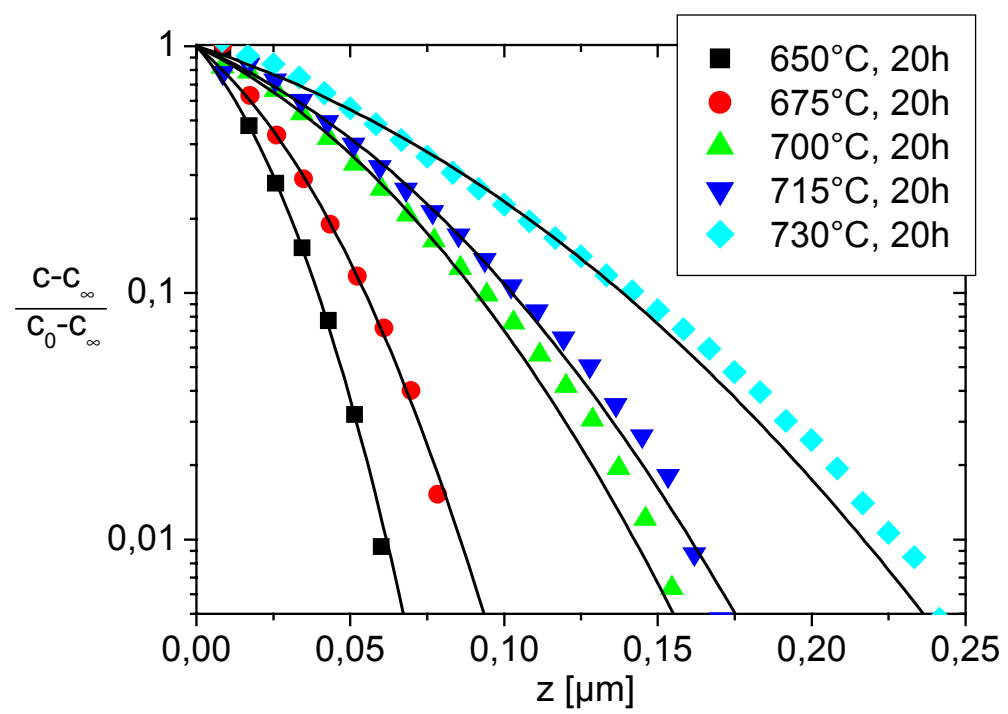

Abb. 6.5: Untergrundkorrigierte, normierte ${ }^{18} \mathrm{O}$-Tracer-Diffusionsprofile in $\mathrm{IrO}_{2}$ Schichten für verschiedene gemessene Temperaturen und einer Glühdauer von $\mathrm{t}=20 \mathrm{~h}$.

Die Diffusionskonzentrationsprofile in Abb. 6.5 sind so dargestellt, dass auf der Ordinate die auf die Oberflächenkonzentration normierte, untergrundkorrigierte Konzentration, also $\left(c-c_{\infty}\right) /\left(c_{0}-c_{\infty}\right)$ aufgetragen ist. Dabei ist $c=c(z)$ die Isotopenkonzentration in der Tiefe z, $\quad c_{\infty}=c(z=\infty)$ die natürlich vorhandene Hintergrund-Isotopenkonzentration und $c_{0}=c(z=0)$ die an der Oberfläche gemessene Konzentration. 
Anhand der in Abb. 6.5 gezeigten Profile der Diffusionsglühungen zwischen $650^{\circ} \mathrm{C}$ und $730^{\circ} \mathrm{C}$ mit identischer Glühdauer von $\mathrm{t}=20 \mathrm{~h}$ wird deutlich, dass die Konzentrationsprofile die erwartete systematische Zunahme der Profilbreite bzw. der Diffusionslänge mit zunehmender Temperatur aufweisen und dass sich der Konzentrationsverlauf sehr gut mit dem gewählten Modell beschreiben lässt. Nur für größere Diffusionslängen und sehr geringe Konzentrationen weichen die Konzentrationen leicht von den angepassten Profilen ab. Als Gründe für die geringe, aber systematische Abweichung kann ein bei SIMS-Analysen bisweilen beobachteter Effekt angeführt werden, bei dem der Sputtereffekt eine selektive Rückstoßimplantation und damit eine Durchmischung der oberen Atomlagen der Probe bewirkt. Dadurch kann für längere Sputterzeiten eine Verfälschung der wahren Konzentration auftreten („Schneepflugeffekt“) [Joh81]. Bei sehr geringen Konzentrationen ist zudem der Verlauf des Isotopenprofiles sehr empfindlich gegenüber der durchgeführten Untergrundkorrektur.

Die Tatsache, dass sich die gemessene Diffusion ausschließlich mit einem Fick'schen Diffusionsmodell beschreiben lässt, deutet darauf hin, dass in den reaktiv gesputterten $\operatorname{IrO} \mathrm{O}_{2}$ Schichten keine Korngrenzen als offene Diffusionspfade wirken. In diesem Fall würde man vielmehr beobachten, dass sich zusätzlich für größere Diffusionslängen ein langgezogenes Konzentrationsprofil ergibt, das durch eine $\ln (c) \propto z^{6 / 5}$-Abhängigkeit beschreibbar ist [Chu96], [Cla63], [Suz64]. Diese Korngrenzendiffusion wird in einigen anderen Oxiden als dominierender Diffusionsmechanismus gefunden, beispielsweise in ultrafeinkörnigem gesinterten $\mathrm{ZrO}_{2}$ [Bro99] und in pulverkompaktiertem nanokristallinen $\mathrm{TiO}_{2}$. [Höf91] Die ausschließliche Existenz der Volumendiffusion ist ein bemerkenswertes Resultat für den Einsatz der $\mathrm{IrO}_{2}$ Schichten als Sauerstoffdiffusionsbarriere, da die Korngrenzendiffusion schon für geringe Temperaturen aufgrund der niedrigen Aktivierungsenergie im allgemeinen mehrere Größenordnungen höher ist als die Volumendiffusion.

Die Arrhenius-Auftragung der Diffusionskoeffizienten, die aus den in Abb. 6.5 präsentierten Profilen und weiteren Diffusionsmessungen errechnet worden sind, ist in Abb. 6.6 gezeigt. Anhand der Daten lässt sich erkennen, dass die Diffusionskoeffizienten im gemessenen Temperaturintervall in Arrhenius-Auftragung sehr gut linear korrelieren, das heißt, sich mittels eines Arrhenius-Gesetzes in der Form

$$
D^{T}=D_{0}^{T} \cdot \exp \left(-E_{A} / k_{B} T\right)
$$

beschreiben lassen. Der in Abb. 6.6 gezeigte Arrhenius-Fit der Diffusionsdaten liefert $D_{0}^{T}=(2,8 \pm 2,5) \cdot 10^{-6} \mathrm{~m}^{2} / \mathrm{s}$ und $E_{A}=(2,73 \pm 0,07) \mathrm{eV}$. Die Fehlerbalken sind anhand der angenommenen Ungenauigkeit in der Glühtemperatur von $d T= \pm 5^{\circ}$ nach der Formel 


$$
\frac{d D^{T}}{D^{T}}=\frac{E_{A}}{k T^{2}} d T
$$

bestimmt worden.

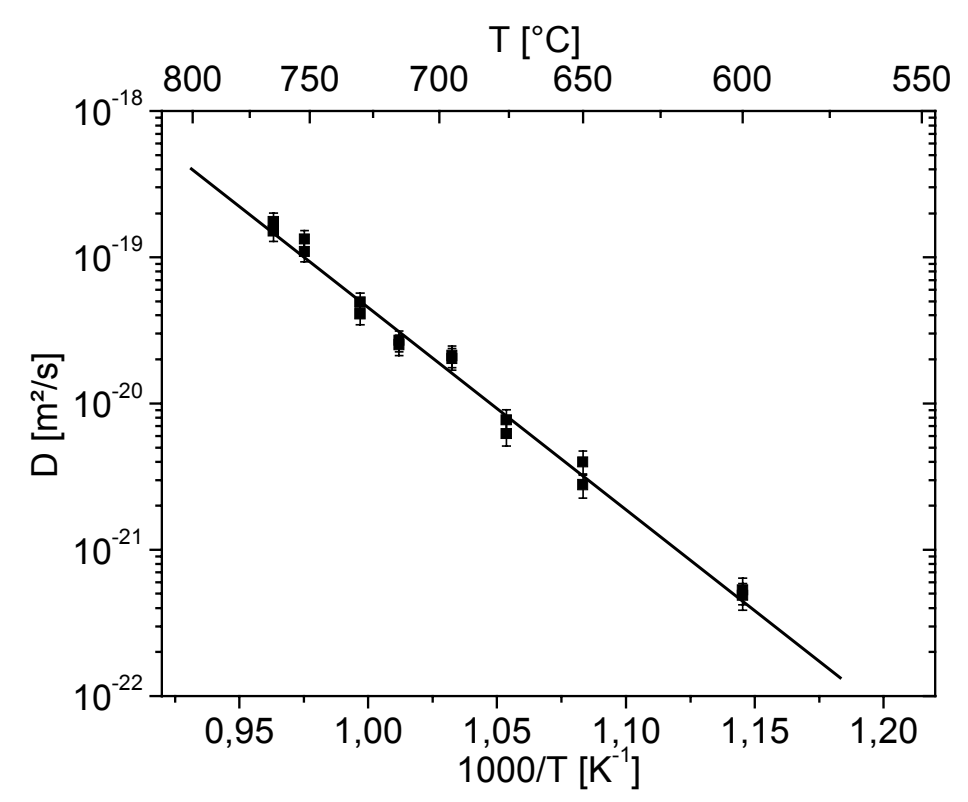

Abb. 6.6: Arrhenius-Auftragung der Sauerstoffisotopendiffusionskoeffizienten $\mathrm{D}^{\top}$ in $\operatorname{IrO}_{2}$ Schichten, die $2 \mathrm{~h}$ bei $800^{\circ} \mathrm{C}$ in ${ }^{16} \mathrm{O}_{2}$ vorgeglüht worden sind. Es wurden Proben mit Schichtdicken von $0,3 \mu \mathrm{m}$ und $0,5 \mu \mathrm{m}$ verwendet, als Substrat wurde thermisch oxidiertes Si benutzt. Die eingezeichnete Gerade stellt die Arrhenius-Anpassung an die Daten dar.

Angesichts des hier ermittelten Wertes für die Aktivierungsenergie $E_{A}$ ist für die Sauerstoffdiffusion $\mathrm{im} \mathrm{IrO}_{2}$ der Leerstellendiffusionsmechanismus als sehr wahrscheinlich anzunehmen, da man für einen interstitiellen Diffusionsmechanismus eine deutlich geringere Aktivierungsenergie erwarten würde. Der Leerstellendiffusionsmechanismus ist ebenfalls geometrisch plausibel, da im $\mathrm{IrO}_{2}$ eine dichteste Kugelpackung der Sauerstoffionen vorliegt, in deren Oktaederlücken die deutlich kleineren Metallkationen angeordnet sind. Diese Struktur lässt nur Metallionen, nicht aber die deutlich größeren Sauerstoffionen auf interstitiellen Plätzen zu [Hau65]. Der Leerstellenmechanismus ist ebenfalls in vielen dichtest gepackten Metallen als dominierender Diffusionsmechanismus gefunden worden [Haa94]. Aufgrund der fast identischen lonenradienverhältnisse des $\mathrm{TiO}_{2}$ und des isostrukturellen $\mathrm{IrO}_{2}$ werden die hier errechneten Werte für den Präexponentialfaktor $D_{0}^{T}$ und für die Aktivierungsenergie $\mathrm{E}_{\mathrm{A}}$ mit Ergebnissen von Sauerstoffdiffusionsmessungen verglichen, die an polykristallinen $\mathrm{TiO}_{2}$ Proben durchgeführt wurden. Trotz der deutlich geringeren Sublimationstemperatur des $1 \mathrm{rO} \mathrm{O}_{2}$ 
zur Schmelztemperatur des $\mathrm{TiO}_{2} 6$ sind für die Sauerstofftracerdiffusion in $\mathrm{TiO}_{2}$ jedoch sehr ähnliche Aktivierungsenergien von $E_{A}=2,60 \mathrm{eV}$ bis $E_{A}=2,87 \mathrm{eV}$ und Werte von $1 \cdot 10^{-7} \mathrm{~m}^{2} / \mathrm{s}<D_{0}^{T}<1,7 \cdot 10^{-6} \mathrm{~m}^{2} / \mathrm{s}$ gefunden worden [Hau65], [Lun78], [Kin76]. Die zum Sauerstofftransport veröffentlichten Ergebnisse im viel untersuchten $\mathrm{TiO}_{2}$ wurden ebenfalls durch einen für die Sauerstoffdiffusion verantwortlichen Leerstellendiffusionsmechanismus im Anionenuntergitter gedeutet [Hau65], [Kim98]. Die Größenverhältnisse der lonen sind für einige tetragonale Metalloxide mit den Sauerstoffionen in dichtester Packung nach zunehmenden Kationenradien geordnet in Tab. 6.3 angegeben. Anhand der dort gezeigten Daten für die als Sauerstoffbarrieren eingesetzten Oxide wird die strukturelle Ähnlichkeit besonders deutlich.

Tab. 6.3: Ionenradien in einigen tetraedrischen Metalloxiden [Gme78], [JCP95]

\begin{tabular}{llllll}
\hline Material & $\begin{array}{l}\text { Kationenradius } \\
\mathbf{r}_{\mathrm{K}}[\AA]\end{array}$ & $\begin{array}{l}\mathbf{r}_{\mathrm{K}} / \mathbf{r}_{\mathrm{O} 2-} \\
{[\AA]}\end{array}$ & $\begin{array}{l}\text { Gitterparameter a } \\
{[\AA]}\end{array}$ & $\begin{array}{l}\text { Gitterparameter c } \\
{[\AA]}\end{array}$ & $\begin{array}{l}\text { Achsen- } \\
\text { verhältnis c/a }\end{array}$ \\
\hline $\mathrm{TiO}_{2}$ & 0,72 & 0,58 & 4,5933 & 2,9592 & 0,64 \\
$\mathrm{RuO}_{2}$ & 0,73 & 0,59 & 4,4994 & 3,1071 & 0,69 \\
$\mathrm{IO}_{2}$ & 0,74 & 0,60 & 4,4983 & 3,1544 & 0,70 \\
$\mathrm{OsO}_{2}$ & 0,75 & 0,61 & 4,5000 & 3,184 & 0,71 \\
\hline
\end{tabular}

\subsection{Diffusionsmessungen an nicht vorgeglühten $\mathrm{IrO}_{2}$ Proben}

Für die Integration der $\mathrm{IrO}_{2}$ Schichten ist es wichtig, das thermische Budget, also die Temperaturbelastung des Barrieren-Stacks, nach der Abscheidung niedrig zu halten. Dies ist nötig, um eine Degradation des Si-plug/Haftschicht-Kontaktes, für die es im wesentlichen drei Gründe gibt, zu vermeiden. Bei hohen Temperaturen $\mathrm{T}>>700^{\circ} \mathrm{C}$ und langen Glühdauern kann es zu einer Diffusion des Siliziums in das vor dem $\mathrm{IrO}_{2}$ abgeschiedene Ir kommen, aus der im allgemeinen eine unerwünschten Bildung von hochohmigen oder nicht leitfähigen Iridiumsilizidphasen resultiert [Pet79], [Sch94], [Sae98]. Die Phasenbildung im Ir-Si System, das in Abb. 6.7 ausschnittsweise dargestellt ist und die die Existenz von 10 intermetallischen stabilen und metastabilen Phasen aufweist [Mae95], ist noch immer Gegenstand aktueller Forschung [Dem99], [Kur97].

\footnotetext{
${ }^{66} \mathrm{~T}_{\mathrm{m}}\left(\mathrm{TiO}_{2}\right)=1855^{\circ} \mathrm{C}[$ Ans98, S.768f. $]$
} 


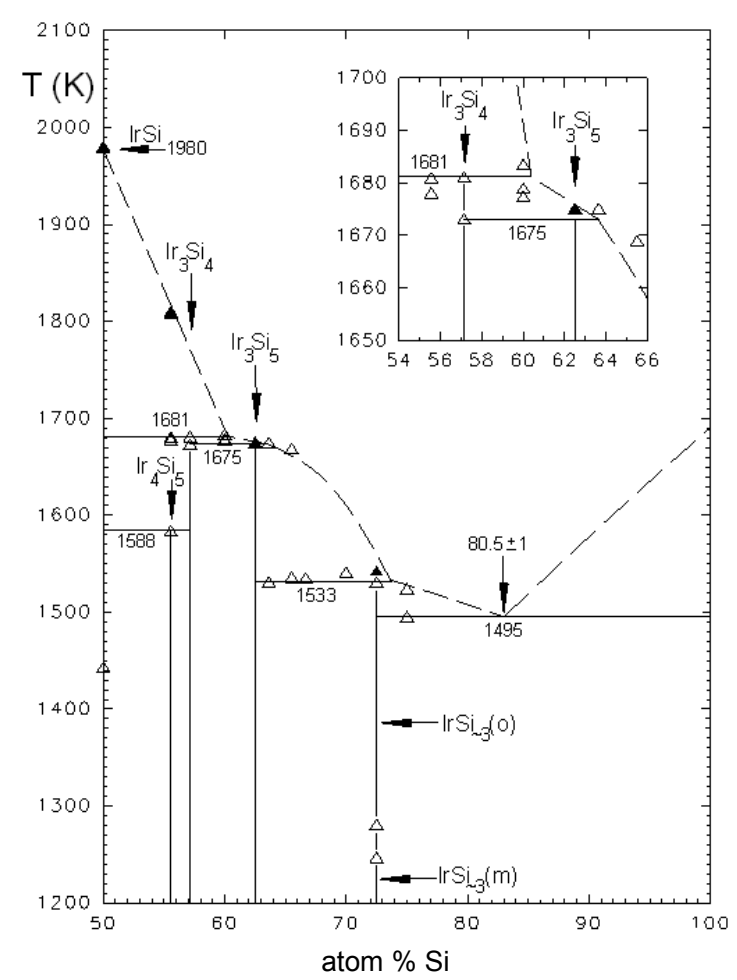

Abb. 6.7: Ausschnitt aus dem Ir-Si Phasendiagramm für Si-reiche Systeme, aus [All93]

Überdies kommt es beim $\mathrm{IrO}_{2}$, wie in Kap. 5.4 gezeigt wurde, im Temperaturbereich über $700^{\circ} \mathrm{C}$ zu einer sehr starken Reduktion der kompressiven Spannungen, die zur Mikrorissbildung in der $\mathrm{IrO}_{2}$ Schicht und auch zur Ablösung des $\mathrm{IrO}_{2}$ von der Ir Schicht führen kann. Als dritter limitierender Aspekt begrenzt das für die Ir Schichten bei hohen Temperaturen gefundene Kornwachstum und die damit verbundene Aufrauung des Schichtstapels die maximal einsetzbare Temperatur. Es wird daher hier untersucht, inwieweit die $\mathrm{IrO}_{2}$ Schichten im abgeschiedenen Zustand („wie hergestellt“) ohne Hochtemperaturvorbehandlung als Sauerstoffdiffusionsbarrieren wirken. Dabei wird angenommen, dass sich die Proben während des Aufheizprozesses auf die Diffusionstemperatur nahezu im Gleichgewicht befinden und die Matrix während der Diffusionsglühung als statisch angesehen werden kann, dass also keine signifikante Erholung oder Gefügeänderung stattfindet. Diese Annahme basiert auf der Tatsache, dass die Relaxation der mechanischen Spannungen für die untersuchten Raten bis $r=10 \mathrm{~K} / \mathrm{min}$ nicht heizratenabhängig ist. Zudem wurde gefunden, dass nach kumulativen isothermen Anlassvorgängen von je 30 Minuten Dauer nach der zweiten und weiteren Temperungen keine Spannungsänderungen an den Schichten mehr nachweisbar waren.

Es wurden Diffusionsglühungen an den „wie hergestellten“ Proben bei Temperaturen von $550^{\circ} \mathrm{C}<\mathrm{T}<700^{\circ} \mathrm{C}$ für eine Glühzeit von einer Stunde durchgeführt. Man beobachtet für diese moderaten Diffusionstemperaturen, dass sich ebenfalls durch das Ficksche Gesetz beschreibbare Tracer-Konzentrationsprofile ergeben. Die quantitative Auswertung führt zu den 
in Abb. 6.8 dargestellten Diffusionskoeffizienten, die etwa um zwei bis drei Größenordnungen über den an vorgeglühten Proben gemessenen Werten liegen.

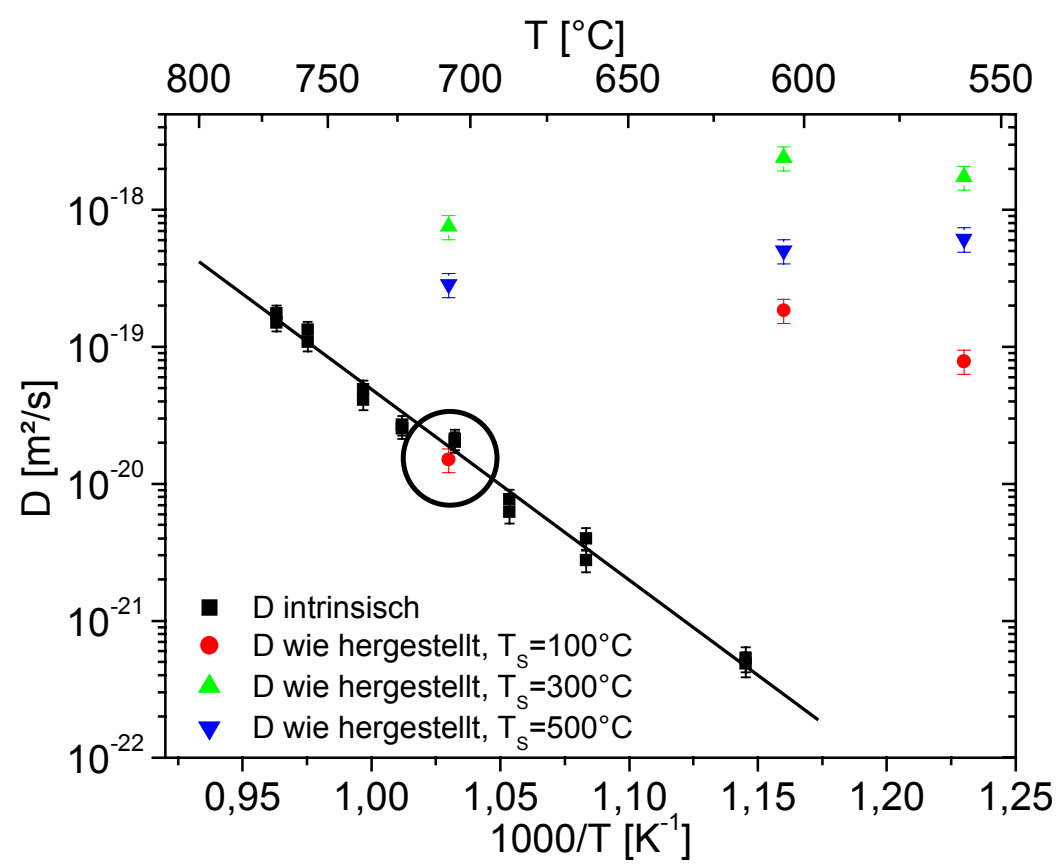

Abb. 6.8: Diffusionskoeffizienten der in ${ }^{16} \mathrm{O}_{2}$ vorgeglühten Proben (Quadrate) und der nicht vorgeglühten Proben (farbige Dreiecke und Kreise). Mit einem Kreis markiert ist der Diffusionskoeffizient in der Probe, die nach dem Aufheizen auf die Diffusionstemperatur bereits den intrinsischen Wert aufweist.

Ein einfacher Erklärungsansatz ist die Annahme, dass die Erhöhung des Diffusionskoeffizienten $D^{T}$ durch eine Erhöhung der Leerstellenkonzentration $c_{L}$ hervorgerufen wird. Bei einer erhöhten Nichtgleichgewichts-Leerstellenkonzentration der $\mathrm{IrO}_{2}$-Proben, die durch den bereits in Kap. 4.4 diskutierten Depositionsprozess verursacht wird, sollte nach der Gleichung [Haa94]

$D(T)=\gamma \cdot a^{2} \cdot \omega(T) \cdot c_{L}$

eine der Leerstellenkonzentration proportionale Erhöhung des Präexponentialfaktors bzw. des Diffusionskoeffizienten zu beobachten sein. Dabei stellt $\gamma$ einen geometrischen Faktor, $a$ die Sprungweite und $\omega$ die Sprungfrequenz des Atoms in die Leerstelle dar. Indem $\omega$ durch einen Boltzmannverteilungsfaktor für die Leerstellenwanderung ausgedrückt wird, lässt sich die Gleichung (6.6) auch in der durch Gleichung (6.4) gegebenen Arrhenius-Form schreiben. Weiterhin wird angenommen, dass die zusätzlichen Leerstellen bzw. FrenkelDefekte, die durch den ballistischen Beschuss bei der Deposition entstanden sind, während des Anlassens auf die Diffusionstemperatur nicht vollständig ausheilen. Anhand der hochauf- 
lösenden Elektronenmikroskopiebilder ist jedoch in den „wie hergestellten“ Proben auch die Existenz zahlreicher höherdimensionaler Defekte wie Versetzungen und Zwillingsgrenzen nachgewiesen worden. Es ist anzunehmen, dass der Diffusionskoeffizient ebenfalls durch diese Defekte erhöht wird, dass aber aufgrund der kompakten Mikrostruktur und der kompressiven Spannungen kein Korngrenzendiffusionsmechanismus aktiv ist, da dieser zu einer im vorigen Kapitel diskutierten, zweigeteilten Profilform mit einer $\ln (c) \propto z^{6 / 5}$-Abhängigkeit für größere Diffusionstiefen führen würde.

Der in Kap. 5diskutierte, beim Aufheizprozess ablaufende Rekristallisationsvorgang ist für die drei gewählten Depositionstemperaturen jedoch signifikant unterschiedlich. Es wird anhand der Diffusionsergebnisse in Abb. 6.8 deutlich, dass bei der kaltgesputterten $\mathrm{IrO}_{2}$ Probe der Rekristallisationsprozess bei einer Diffusions-Temperatur von $700^{\circ} \mathrm{C}$ so weit fortgeschritten ist, dass die die Diffusion beschleunigenden Defekte ausgeheilt sind. In diesem Fall beobachtet man nicht mehr, wie bei allen anderen Proben, die extrinsische Diffusion, sondern vielmehr die bereits in Abb. 6.6 gezeigten, für $700^{\circ} \mathrm{C}$ gemessenen, intrinsischen Werte. Die Diffusionskoeffizienten der heißgesputterten Proben $\left(T_{S}=300^{\circ} \mathrm{C}\right.$ und $\left.500^{\circ} \mathrm{C}\right)$ liegen bei $700^{\circ} \mathrm{C}$ etwa 1 bis 2 Größenordnungen höher. Man erkennt für alle nicht vorgeglühten Proben, dass sich kein konsistentes Arrheniusverhalten ergibt, da die Proben unterschiedliche Matrizes mit zu höheren Temperaturen abnehmender Defekt- bzw. Leerstellendichte besitzen.

\subsection{Sauerstoffdiffusion in Ir Proben}

Es soll weiterhin gezeigt werden, dass in der in Kap. ฤuntersuchten $\mathrm{IrO}_{2} / \mathrm{lr}$ Doppelschichtstruktur nicht die Ir Schicht die Rolle der Diffusionsbarriere übernimmt, sondern vielmehr zur Vermeidung der Reduktion der $\mathrm{IrO}_{2}$ Schicht dient. Ein direkter Kontakt des $\mathrm{IrO}_{2} \mathrm{zu}$ einer Nitridschicht (z.B. TiN), die gewöhnlich als Siliziumdiffusionsbarriere eingesetzt wird [Jim91], würde beim Anlassen zu einer Reduktion des $\mathrm{IrO}_{2}$ und zu einer Oxidbildung der Nitridschicht gemäß

$$
\mathrm{IrO}_{2}+\mathrm{TiN} \rightarrow \mathrm{Ir}+\mathrm{TiO}_{2}+1 / 2 \mathrm{~N}_{2} \quad \Delta H=-366,9 \mathrm{~kJ} / \mathrm{mol}
$$

führen [Kub79].

In Analogie zur Sauerstoffdiffusion in $\mathrm{IrO}_{2}$ Schichten wurden Sauerstoffkonzentrationsprofile in den bei Temperaturen zwischen $550^{\circ} \mathrm{C}$ und $700^{\circ} \mathrm{C}$ geglühten Ir Schichten mittels SIMS aufgenommen und ausgewertet. Die in Sauerstoff geglühten Schichten weisen ein Diffusionsprofil auf, dass sich in zwei Teile separieren lässt; in Abb. 6.9 ist ein Diffusionsprofil der bei $600^{\circ} \mathrm{C}$ geglühten Probe gezeigt. An der Oberfläche ist ein wenige Nanometer dickes, schnell abfallendes Profil nachweisbar, für größere Diffusionstiefen wird ein langgezogenes Konzentrationsprofil beobachtet. Die Interpretation der gemessenen Konzentrationsverläufe 
im Sinne einer gemischten Diffusion, die an der Oberfläche durch die Volumendiffusion bestimmt und in der Tiefe durch die Korngrenzendiffusion dominiert wird [Whi54], [Suz61], erscheint a priori möglich, jedoch ergibt eine solche Auswertung eine unphysikalisch hohe Aktivierungsenergie für die Korngrenzendiffusion und eine unverständlich niedrige Aktivierungsenergie für die Volumendiffusion, so dass diese Interpretation als falsch angesehen werden muss. Da sich mittels Röntgendiffraktometrie bei der bei $700^{\circ} \mathrm{C}$ in Sauerstoff getemperten Ir Schicht intensitätsschwache $\mathrm{IrO}_{2}$ Reflexe nachweisen lassen, müssen die Diffusionsprofile anders gedeutet werden: Der erste Teil des Profils der bei hohen Temperaturen in Sauerstoff geglühten Proben wird demnach der partiellen Oberflächenoxidation des Ir zugeschrieben, da die mit der SIMS gemessene lonenausbeute im allgemeinen stark matrixabhängig ist und dadurch eine Konzentrationserhöhung der ersten Oberflächenschichten vortäuscht. Das langgezogene Tiefenprofil lässt sich mit einer Fehlerfunktion beschreiben und wird somit der Volumendiffusion des Sauerstoffs im Ir zugeordnet.

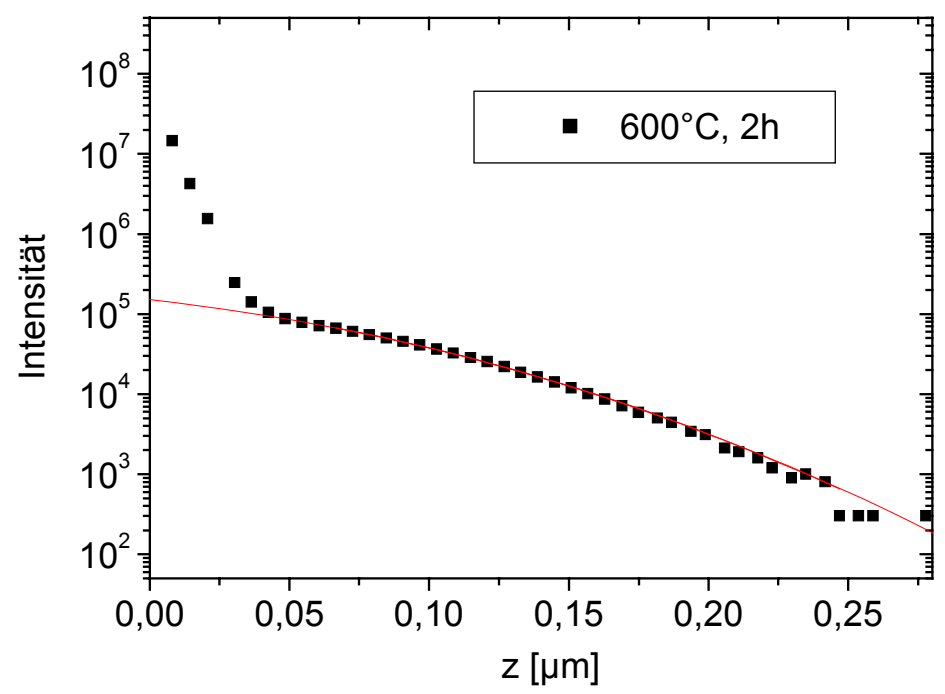

Abb. 6.9: SIMS Sauerstoffprofil in einer Ir Schicht nach Glühung bei $600^{\circ} \mathrm{C}$ für $2 \mathrm{~h}$ in Sauerstoffatmosphäre. Die eingezeichnete Funktion stellt die angepasste Fehlerfunktion an den Konzentrationsverlauf dar, die Signalerhöhung an der Oberfläche wird der partiellen Oxidation der Oberfläche zugeordnet.

Für die Auswertung wurde angenommen, dass die unvollständige oberflächliche Oxidation, die nach der kurzen Glühzeit bei den untersuchten Glühtemperaturen auftritt [Peu84], [Gme78], keinen signifikanten Einfluss auf die Lösung der Differentialgleichung mit der Randbedingung der konstanten Oberflächenkonzentration hat. Diese Annahme stützt sich auf feldionenmikroskopische Analysen an thermisch in Sauerstoff behandelten Ir Proben, die für Temperaturen unterhalb von $600^{\circ} \mathrm{C}$ eine sehr hohe Oxidationsbeständigkeit der 
Ir Oberflächen zeigen [Gme78]. Sauerstoffbehandlungen bei $600^{\circ} \mathrm{C}$ führen nur zu einer partiellen Oxidation der Oberfläche [For68].

Die so bestimmten Sauerstoff-Diffusionskoeffizienten sind zusammen mit den Daten der intrinsischen Sauerstoffdiffusion in $\mathrm{IrO}_{2}$ in Arrhenius-Darstellung in Abb. 6.10 aufgetragen. Es ergibt sich durch eine Arrhenius-Anpassung eine Aktivierungsenergie von $E_{a}=(2.26 \pm 0.40) \mathrm{eV}$ und einem Wert für $D_{0}=5.5 \cdot 10^{-6} \mathrm{~m}^{2} \mathrm{~s}^{-1}$. Die lineare Korrelation der Daten und die realistische Aktivierungsenergie deuten darauf hin, dass die gemachte Annahme für kurze Anlassdauern gerechtfertigt ist, der Oberflächenbereich allerdings für die mathematische Anpassung ausgeschlossen werden muss. Für längere Glühzeiten ist diese Näherung nicht mehr ohne Einschränkung gültig, sondern es ist eine Auswertung nach dem Modell von Wagner [Wag33] notwendig, das die Diffusion des Sauerstoffs durch das gewachsene Oxid berücksichtigt. Der Messwert der bei $700^{\circ} \mathrm{C}$ ausgelagerten Schicht ist daher in Abb. 6.10 in Klammern eingezeichnet.

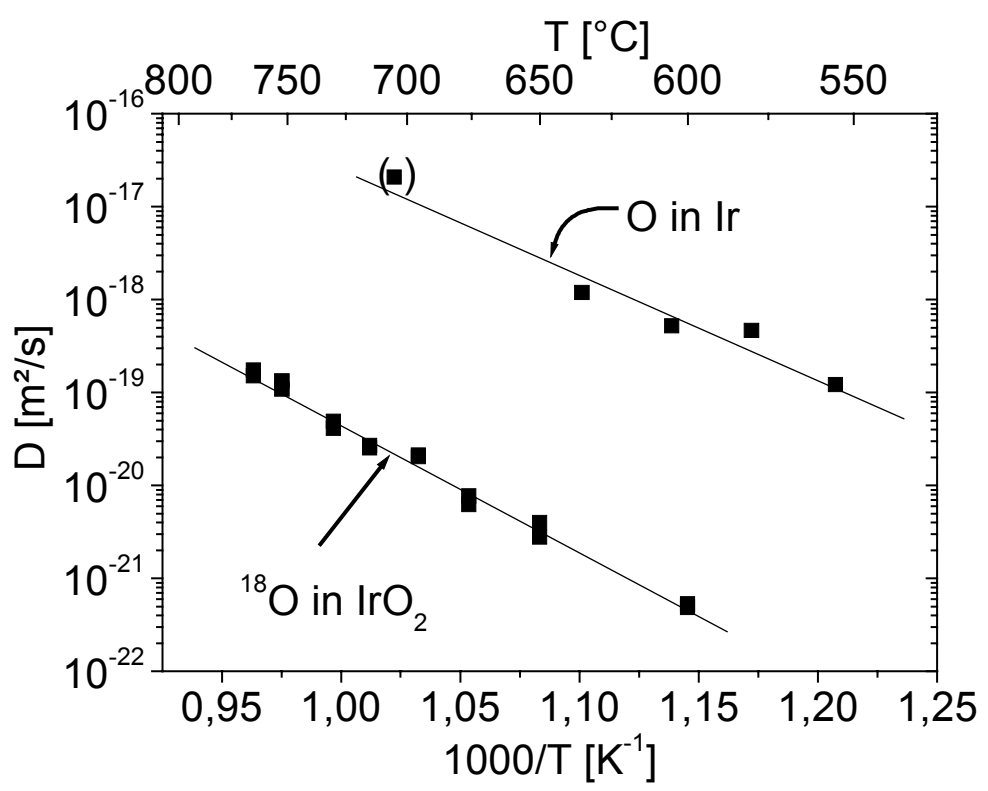

Abb. 6.10: Vergleich der gemessenen ${ }^{16} \mathrm{O}$-Diffusionsdaten in den Ir Schichten mit den ${ }^{18} \mathrm{O}-$ Sauerstofftracerdiffusionsdaten in den $\mathrm{IrO}_{2}$ Schichten. Die eingezeichneten Geraden stellen die Arrhenius-Anpassungen an die Daten dar.

Anhand der in Abb. 6.10 gezeigten Daten wird deutlich, dass die Sauerstoffdiffusion in den $\mathrm{IrO}_{2}$ Schichten in dem untersuchten Temperaturbereich etwa drei Größenordnungen geringer ist als in den Ir Schichten. Um die Diffusion vergleichen zu können, muss die Tatsache berücksichtigt werden, dass die ${ }^{16} \mathrm{O}$ Diffusion in den $\mathrm{IrO}_{2}$ Schichten aufgrund des Isotopieeffektes gemäß der Gleichung 


$$
\frac{D\left({ }^{16} O\right)}{D\left({ }^{18} O\right)}=\frac{\omega\left({ }^{16} O\right)}{\omega\left({ }^{18} O\right)}=\frac{\sqrt{m\left({ }^{18} O\right)}}{\sqrt{m\left({ }^{16} O\right)}}=\sqrt{18 / 16} \approx 1,06
$$

um etwa $6 \%$ größer als die ${ }^{18} \mathrm{O}$ Diffusion sein sollte. Für Gleichung (6.7) sind die gleichen Bezeichnungskonventionen wie in Gleichung (6.6) gewählt und bei (*) ist angenommen worden, dass die gleichen Matrixeigenschaften wie für die ${ }^{18} \mathrm{O}$ Diffusion gelten. Spannungsmessungen an den Ir Schichten haben gezeigt, dass die kompressiven Schichtspannungen während des Aufheizens über $500^{\circ} \mathrm{C}$ vollständig relaxiert sind, daher werden die gemessenen Sauerstoffdiffusionskoeffizienten im Ir als intrinsisch angesehen. Die hier beobachtete Sauerstoffisotopendiffusion in durch den Sputterprozess geschädigten $\mathrm{IrO}_{2}$ Schichten ist bei Glühtemperaturen von etwa $600^{\circ} \mathrm{C}$ vergleichbar mit der intrinsischen Sauerstoffdiffusion in den Ir Schichten.

Es wird aus der signifikant höheren Sauerstoffdiffusion in den gesputterten Ir Schichten in dem untersuchten Temperaturintervall gefolgert, dass die Ir Schichten nicht als eigenständige Sauerstoffbarriere für eine Sauerstoffbehandlung bei $700^{\circ} \mathrm{C}$ ausreichend sind. Eine einfache Abschätzung des mittleren Verschiebungsquadrats $\left\langle x^{2}\right\rangle=2 D t$ der Sauerstoffdiffusion in den Ir Schichten ergibt für eine Temperatur von $700^{\circ} \mathrm{C}$ und für eine Anlassdauer von zwei Stunden einen Wert von etwa $\left\langle x^{2}\right\rangle=0,5 \mu \mathrm{m}$. Dieser Wert für die minimal erforderliche Ir Schichtdicke ist aus Integrationsgründen für den in Kap. 1 genannten 4Mbit-Chip jedoch nicht realisierbar, da die für die Strukturgebung der Barriere notwendige Rückätzung eine zur Schichtdicke proportionale Strukturaufweitung mit sich bringt [Yun98]. Zudem ist die beobachtete Aufrauung der Ir Schichten während des Anlassens in Sauerstoffatmosphäre ein weiterer Aspekt, der die Anwendbarkeit der Ir Schichten auf niedrigere Temperaturen beschränkt [Sae01]. 


\section{Integration der $\mathrm{IrO}_{2}$ Sauerstoffbarriere}

\subsection{Stabilität von planaren Schichtstapeln}

Die Stabilität unstrukturierter $\mathrm{IrO}_{2} / \mathrm{lr}$ Barrierenschichtstapel soll mittels Elektronenmikroskopie und AES-Tiefenprofilanalysen untersucht werden. Dazu wurden analog zu der 1997 von Yamamichi et al. [Yam97] vorgestellten, allerdings auf $\mathrm{RuO}_{2} / \mathrm{Ru}$ basierenden Barrierenstruktur, sequentiell auf ganzflächig mit poly-Silizium beschichteten Scheiben eine Ti/TiN Doppelschicht und eine $\mathrm{Ir} / \mathrm{rO}_{2}$ Doppelschicht abgeschieden. Auf diesen Schichtstapel wurde schließlich die Pt Elektrode gesputtert. Die Ti Schicht dient in der beschriebenen Schichtabfolge als Adhäsionsvermittler, die TiN Schicht als Si-Diffusionsbarriere, um eine Iridiumsilizidbildung zu verhindern [Jim91]. Das Ir übernimmt die Rolle einer Pufferschicht, um den direkten Kontakt zwischen dem $\mathrm{IrO}_{2}$ und dem TiN zu vermeiden, der beim Anlassen zur Bildung einer isolierenden Titanoxidschicht führen würde [Ans98].

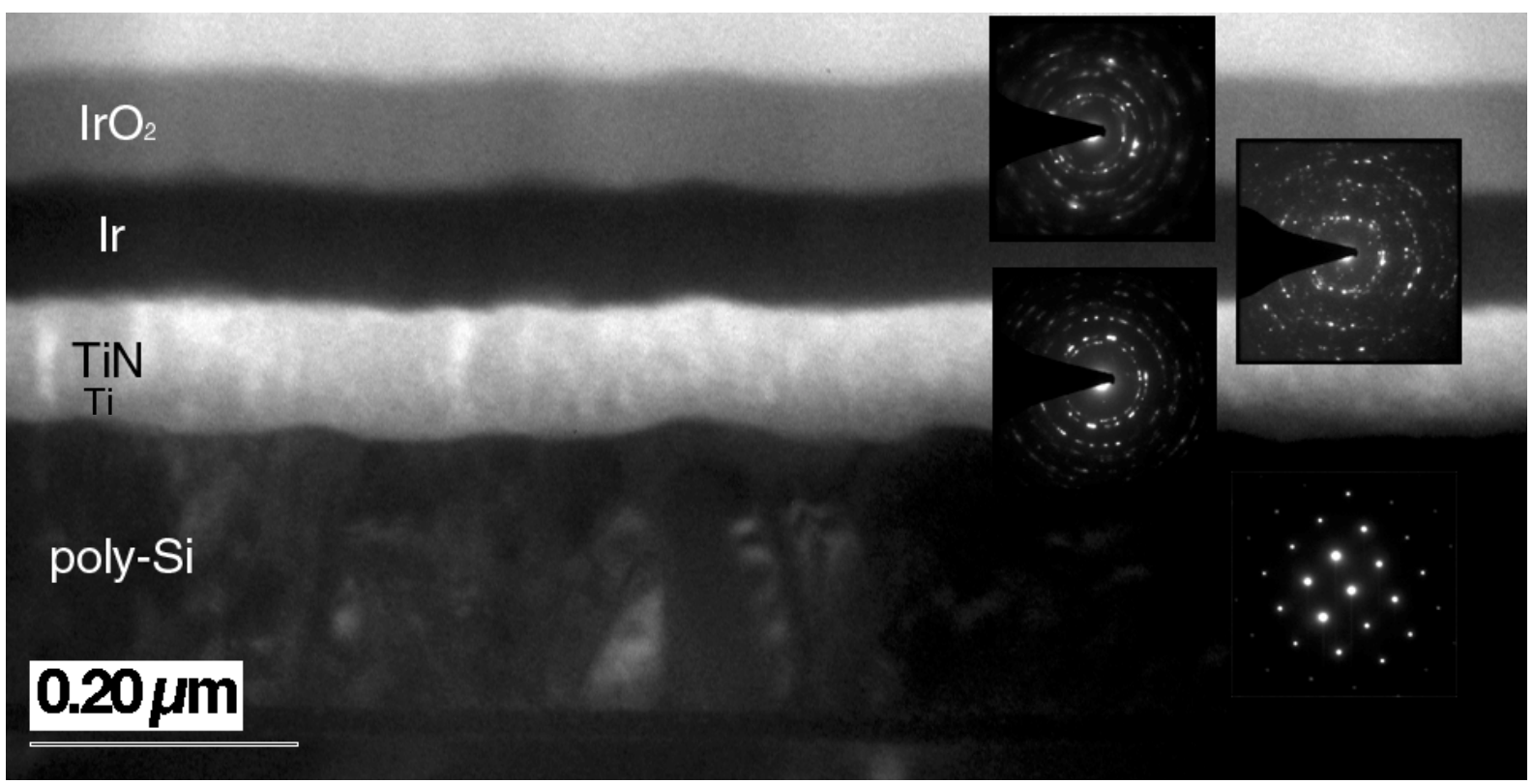

Abb. 7.1: XTEM-Aufnahme der präparierten Probe $\left(\mathrm{Pt} / / \mathrm{rO} \mathrm{O}_{2} / \mathrm{Ir} / \mathrm{TiN} / \mathrm{Ti} / \mathrm{poly}-\mathrm{Si}\right)$ auf oxidiertem SiSubstrat vor der Temperaturbehandlung. Eingefügt sind Elektronenbeugungsbilder der gezeigten Schichten.

Anhand der hier vorgestellten Schichtstruktur soll der Einfluss der Abscheidetemperatur des stöchiometrischen $\mathrm{IrO}_{2}$ auf die Stabilität des gesamten Barrierenschichtstapel untersucht werden. Dazu wurde ein Barrierenschichtstapel hergestellt, bei dem die $\mathrm{IrO}_{2}$ Schicht bei $\mathrm{T}_{\mathrm{S}}=100^{\circ} \mathrm{C}$ gesputtert wurde (Probe 1) und ein analoger, bei dem die $\mathrm{IrO}_{2}$ Abscheidung bei einer Substrattemperatur von $\mathrm{T}_{S}=500^{\circ} \mathrm{C}$ erfolgte (Probe 2). Eine transmissionselektronenmikroskopische Aufnahme des Probenquerschnitts der Probe 1 ist in Abb. 7.1 dargestellt. Die Ti Schicht hat eine Dicke von nur $20 \mathrm{~nm}$ und ist in der hier dargestellten Vergrößerung durch 
den geringen Kontrast zur TiN Schicht kaum zu erkennen. Die Schichtdicken der TiN-, Irund $\mathrm{IrO}_{2}$ Schicht betragen jeweils $100 \mathrm{~nm}$. Anhand der in Abb. 7.1 eingefügten Elektronenbeugungsbilder ist die Kristallinität der Schichten deutlich zu erkennen, besonders die Ir Schicht zeigt eine sehr feinkörnige Mikrostruktur. Die Rauigkeit des Schichtstapels wird maßgeblich durch die sehr großen Körner der poly-Si Schicht beeinflusst.

Für die Untersuchung der thermischen Stabilität des in Abb. 7.1 gezeigten Schichtstapels wurden die zwei so präparierten Proben für 30 Minuten bei $700^{\circ} \mathrm{C}$ in reiner Sauerstoffatmosphäre ausgelagert. Die AES-Spektren der Probe 1 und Probe 2 nach der Temperaturbehandlung zeigt Abb. 7.2.

a)

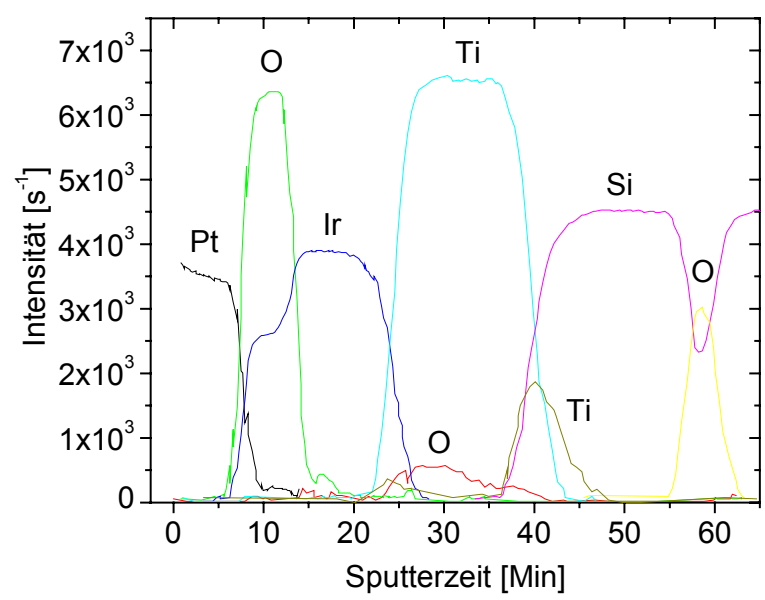

b)

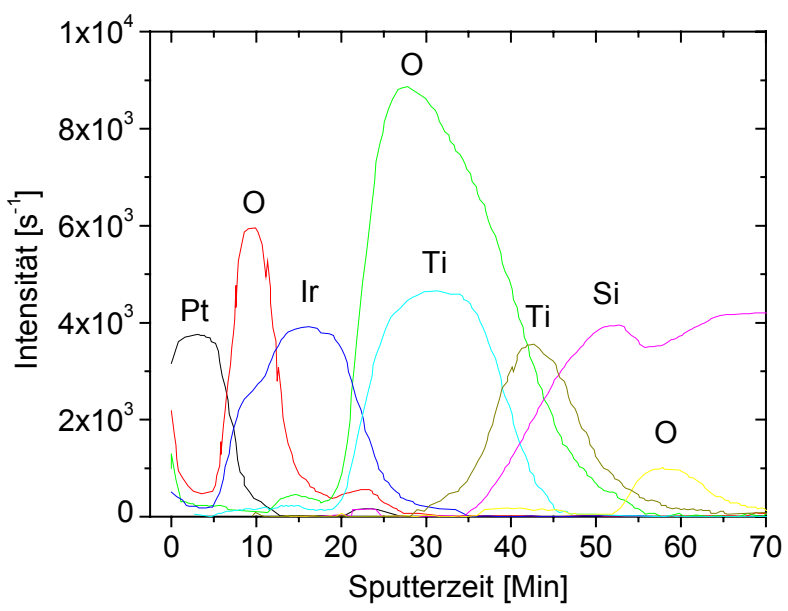

Abb. 7.2: AES-Tiefenprofile der bei $700^{\circ} \mathrm{C}$ in Sauerstoff getemperten Proben, a) Probe 1, bei der die $\mathrm{IrO}_{2}$ Schicht bei $\mathrm{T}_{\mathrm{S}}=100^{\circ} \mathrm{C}$ gesputtert wurde, b) Probe 2, bei der die $\mathrm{IrO}_{2}$ Schicht bei $\mathrm{T}_{\mathrm{S}}=500^{\circ} \mathrm{C}$ gesputtert wurde.

Abb. 7.2 a) verdeutlicht, dass keine signifikante Degradation der Struktur nach der thermischen Behandlung stattgefunden hat. Das Tiefenspektrum zeigt die Existenz scharfer Grenzflächen, das $\mathrm{IrO}_{2}$ hat die Oxidation des TiN erfolgreich verhindert und es hat keine signifikante Interdiffusion bzw. chemische Reaktion stattgefunden. Der geringe in der Ti und TiN Schicht nachgewiesene Sauerstoffgehalt ist nicht durch den Abscheideprozess des Ti(N) oder durch Sauerstoffwiederbedeckung der Schichten während des Messzyklus der AugerAnalyse bedingt, da in den Ausgangsproben vor der Sauerstofftemperung nahezu kein Sauerstoff in den Schichten nachgewiesen werden kann. Es ist zu vermuten, dass eine geringe Menge an Sauerstoff aus der leicht sauerstoff-überstöchiometrischen $\mathrm{IrO}_{2}$ Schicht ausdiffundiert ist und zu einer $\mathrm{TiO}_{x}$ Bildung geführt hat. Der Einfluss des ausdiffundierten Sauerstoffs auf die elektrische Leitfähigkeit des TiN ist jedoch unkritisch, wie in Kap. 7.3 durch elektrische Messungen belegt wird. In die Ti Schicht ist deutlich Si eindiffundiert, das TiN hat jedoch die weitere Diffusion des Si in die Ir Schicht unterbunden, so dass es nicht zu einer Iridiumsilizidbildung gekommen ist. Die Stabilität dieser Struktur ist ebenfalls nach 60 und 
nach 90 Minuten ohne signifikante Degradation bzw. Oxidation der TiN Schicht mittels AESTiefenprofilanalysen nachgewiesen worden. Diese hohe thermische Stabilität kann als ausreichend für die Integration des ferroelektrischen SBT-Materials und erst recht für PZT Schichten angesehen werden [Böt01].

Das in Abb. 7.2 b) dargestellte Tiefenspektrum der Probe 2 hingegen zeigt deutlich, dass die bei $500^{\circ} \mathrm{C}$ gesputterte $\mathrm{IrO}_{2}$ Schicht während der $700^{\circ} \mathrm{C}$-Behandlung nicht als Sauerstoffbarriere gewirkt hat, da die unterliegende TiN und Ti Schicht komplett aufoxidiert ist. Zusätzlich ist $\mathrm{IrO}_{2}$ an der Oberfläche nachweisbar. Um die Reaktion genauer zu untersuchen, wurde eine ortsaufgelöste chemische Oberflächenanalyse durchgeführt.

a)

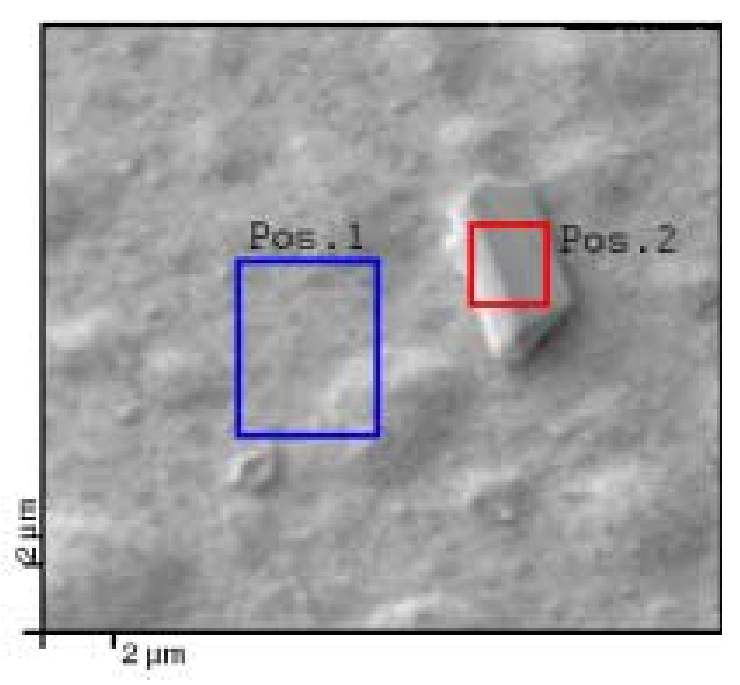

b)

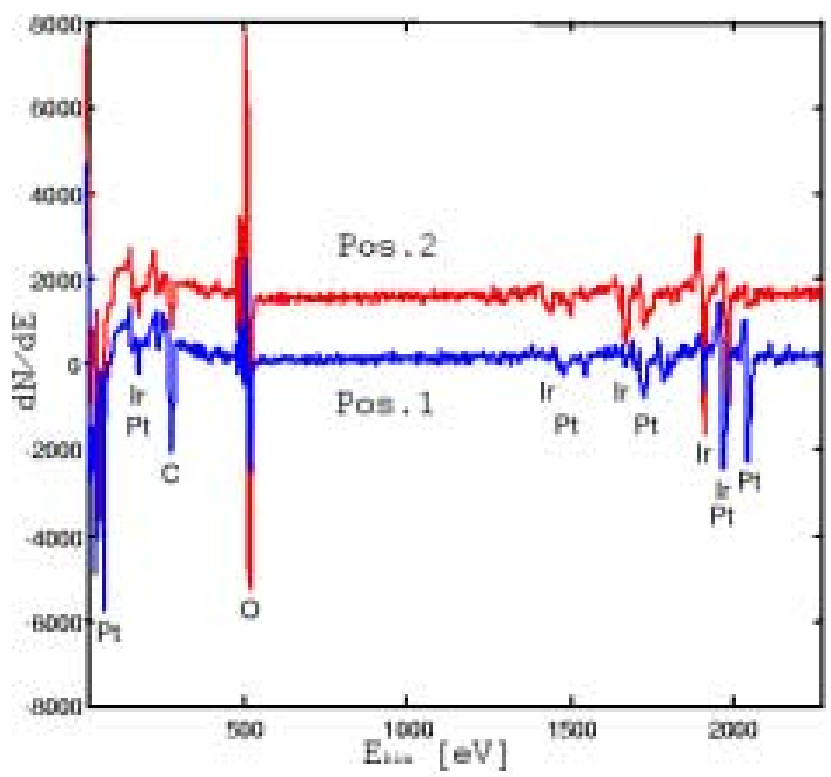

Abb. 7.3: Ortsaufgelöste Oberflächenanalyse des Schichtstapels, bei dem das $\mathrm{IrO}_{2}$ heiß gesputtert wurde $\left(\mathrm{T}_{\mathrm{S}}\left(\mathrm{IrO}_{2}\right)=500^{\circ} \mathrm{C}\right.$, Probe 2) nach der $700^{\circ} \mathrm{C}$ Temperaturbehandlung. a) zeigt eine REM-Analyse der Oberfläche und b) die zugehörigen AES-Spektren.

Die Oberflächenanalyse mittels REM zeigt, dass eine starke Aufrauung des Schichtstapels stattgefunden hat. Die Pt Schicht zeigt mehrere Mikrometer große blasenartige Aufwölbungen, zusätzlich ist die Existenz von ebenfalls mehreren Mikrometern großen Kristallen sichtbar. Diese Kristalle werden wie in Abb. 7.3 b) gezeigt als $\mathrm{IrO}_{2}$ identifiziert. Diese Ergebnisse werden als Konsequenz der Oxidation des TiN interpretiert. Die Oxidation führt zu einer Volumenausdehnung des TiN und einer Freisetzung des Stickstoffs. Da die auf dem TiN befindliche Ir Schicht keine Nitridverbindung bildet, führt die Ansammlung des Stickstoffs zu der Ausbildung von Gasbläschen und resultiert in einem lokalen Abheben der Ir Schicht. Werden die dadurch erzeugten Spannungen zu groß, reißt die Pt Schicht auf und das $1 \mathrm{rO} \mathrm{O}_{2}$ gelangt an die Oberfläche, wo es zu einem $\mathrm{IrO}_{2}$ Kornwachstum kommt. Ähnliche Ergebnisse bezüglich der Degradation sind an $\mathrm{RuO}_{2} / \mathrm{Ru}$ Schichtstapeln von Matsui et al. [Mat00] gefunden worden, die bei $700^{\circ} \mathrm{C}$ für 15 Minuten in Sauerstoff ausgelagert worden sind. Matsui et 
die bei $700^{\circ} \mathrm{C}$ für 15 Minuten in Sauerstoff ausgelagert worden sind. Matsui et al. verwendeten jedoch eine $200 \mathrm{~nm}$ dicke $\mathrm{RuO}_{2}$ und eine $50 \mathrm{~nm}$ dicke Ru Schicht.

Die hier diskutierten Ergebnisse zeigen, dass die Rekristallisation der $\mathrm{IrO}_{2}$ Schicht und die damit verbundene Reduktion der Sauerstoffdiffusion auf den in Kap.6.3 bestimmten, intrinsischen Wert bei einer für die Kristallisation der ferroelektrischen Schicht notwendigen, hohen Anlasstemperatur von $700^{\circ} \mathrm{C}$ die notwendige Voraussetzung darstellt, um die Stabilität des Barrierenschichtstapels zu gewährleisten. Bei einer nicht rekristallisierten, durch den Sputterprozess geschädigten $\mathrm{IrO}_{2}$ Schicht führt die Sauerstoffpenetration bei einer Temperatur von $700^{\circ} \mathrm{C}$ zu einer Oxidation der darunter liegenden Kontaktschichten und damit zu einer Zerstörung des Barrierenschichtstapels.

\subsection{Lithographisch strukturierte Barrierenstacks}

Bei der Integration der Sauerstoff-Barrierenschichten gibt es prinzipiell zwei Möglichkeiten der Realisation. Einerseits ist eine planare Abscheidung aller Schichten inklusive des Ferroelektrikums möglich, so dass die kritischen Hochtemperaturschritte zur Kristallisation der ferroelektrischen Schicht vor der Strukturierung der Barriere erfolgen. Durch dieses sogenannte zweidimensionale Konzept, das nur die zum Substrat parallele Oberfläche der Elektrode ausnutzt, würde man eine Diffusion entlang von geätzten Grenzflächen a priori ausschließen. Ein weiteres Integrationskonzept sieht eine Strukturierung der Barriere vor den kritischen Hochtemperaturschritten vor. Die Motivation dieses zweiten Konzepts ist eine Vereinfachung der Ätzprozesse und eine Vergrößerung der effektiven Kapazität des Kondensators durch die zusätzliche Nutzung der Seitenflächen. Daher soll dieses Konzept auch im folgenden als dreidimensionales Integrationskonzept bezeichnet werden. Dieses letztgenannte Konzept ist geeignet, um besonders hohe Integrationsdichten realisieren zu können [Sch01]. Für die Integration ist es unabdingbar, den dominierenden Diffusionsmechanismus zu kennen, um die Oxidation der Kontaktschicht bzw. des Si-plugs erfolgreich zu unterbinden. Dabei gibt es prinzipiell die in Abb. 7.4 dargestellten Möglichkeiten der Diffusion durch die Barrierenschicht (1) oder die Diffusion entlang von Grenzflächen (2). Dazu gehören sowohl vertikal geätzte Grenzflächen als auch horizontale Grenzflächen zwischen zwei Schichten mit schwacher chemischer Bindung oder schlechter Adhäsion. Die verschiedenen möglichen Diffusionspfade einer strukturierten Barriere sind schematisch in Abb. 7.4 dargestellt. 


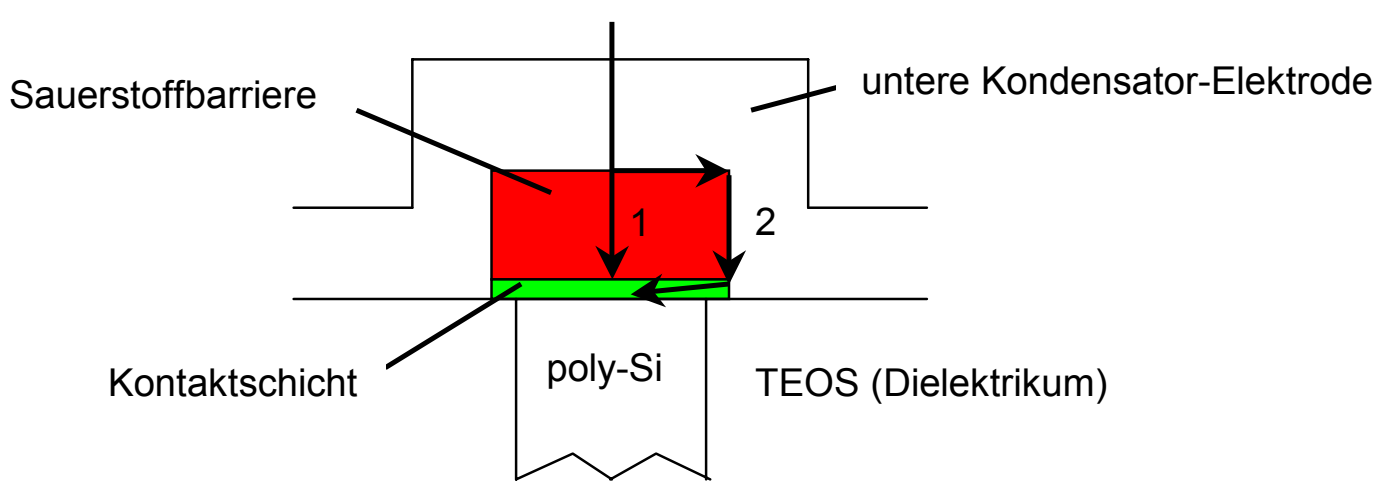

Abb. 7.4: Mögliche Sauerstoff-Diffusionspfade im dreidimensionalen Konzept (1: direkte Diffusion durch die Barrierenschicht, 2: Diffusion entlang von geätzten Grenzflächen)

Um den dominierenden Diffusionsmechanismus zu ermitteln, der den Diffusionspfad für den Sauerstoff darstellt, wurden grundlegende Experimente zur Oxidation der unter der Sauerstoffbarriere liegenden Kontaktschicht durchgeführt. ${ }^{67}$ Dazu wurde auf oxidierten SiSubstraten eine $25 \mathrm{~nm}$ dicke TaN Kontaktschicht aufgesputtert und ohne Brechen des Vakuums sequentiell eine $50 \mathrm{~nm}$ dicke $\mathrm{Ir}$ - und eine $50 \mathrm{~nm}$ dicke optimierte $\mathrm{IrO}_{2}$ Schicht deponiert. Es wurden oxidierte Wafer benutzt, um ein Sauerstoff-Endpunktsignal für den Ätzprozess zu bekommen, mit dem der Schichtstapel strukturiert wird. TaN wurde als Kontaktmaterial gewählt, da es bei einer Oxidation eine sehr ausgeprägte Dickenzunahme zeigt, die leicht mittels Rasterelektronenmikroskopie nachgewiesen werden kann [Ans98]. Dabei benutzt man unter Vernachlässigung der Massenänderung während der Oxidation die Reziprozität von Dichte und Dicke.

$$
d\left(\mathrm{Ta}_{2} \mathrm{O}_{5}\right)=\frac{\rho(\mathrm{TaN})}{\rho\left(\mathrm{Ta}_{2} \mathrm{O}_{5}\right)} \cdot d(\operatorname{TaN})=2,2 \cdot d(\mathrm{TaN})
$$

Der Schichtstapel wurde mittels Lithographie und einem reaktiven Plasmaätzprozess auf eine laterale Strukturgröße von 1,2 $\mu$ m strukturiert und es wurde anschließend eine $150 \mathrm{~nm}$ dicke $\mathrm{Si}_{3} \mathrm{~N}_{4}$ Schutzschicht abgeschieden, die die Oxidation der unterliegenden Schichten verhindert [Pam01], [Tro01]. Die so hergestellte Struktur zeigt Abb. 7.5a) schematisch, der Schichtstapel mit dem geöffneten Siliziumnitrid ist in Abb. 7.5 b) dargestellt. Zusätzlich wird der Schichtstapel $\mathrm{Si}_{3} \mathrm{~N}_{4} / \mathrm{IrO} \mathrm{O}_{2} / \mathrm{Ir} / \mathrm{TaN}$ als Referenz unstrukturiert, aber mit geöffnetem $\mathrm{Si}_{3} \mathrm{~N}_{4}$ mitgetempert, um sicherzustellen, dass das $\mathrm{IrO}_{2}$ durch den Ätzprozess, der für das Öffnen des $\mathrm{Si}_{3} \mathrm{~N}_{4}$ nötig ist, nicht geschädigt wird.

\footnotetext{
${ }^{67}$ Diese Experimente sind in Zusammenarbeit mit Herrn Dr. M. Röhner und Herrn Dr. I. Kasko (Infineon Technologies AG) durchgeführt worden
} 
a)

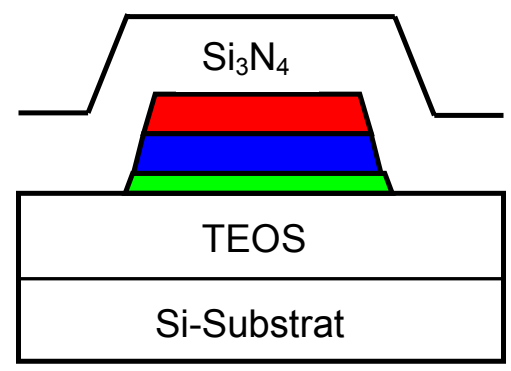

$\mathrm{O}_{2}$

b)

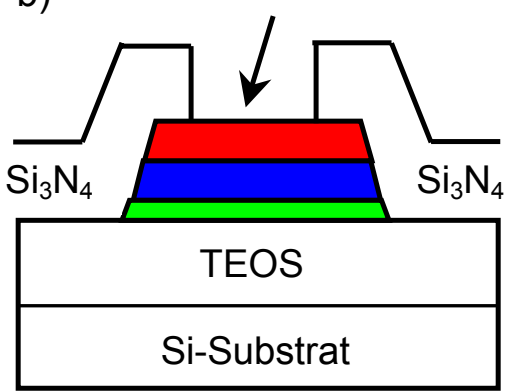

Abb. 7.5: Schematische Abfolge der Schichten zur Analyse des dominierenden Diffusionsmechanismus (grün: TaN, blau: Ir, rot: $\mathrm{IrO}_{2}$ )

Die beschriebenen Proben sind bei $650^{\circ} \mathrm{C}$ in Sauerstoff für eine Dauer von 30 Minuten thermisch nachbehandelt worden. Das Anlasstemperatur und -dauer wurde so gewählt, um einerseits den Oxidationseffekt klar sichtbar zu machen, aber gleichzeitig eine vollständige Degradation der Strukturen durch Abplatzen der oxidierten TaN Schichten bei noch höheren Temperaturen zu vermeiden.
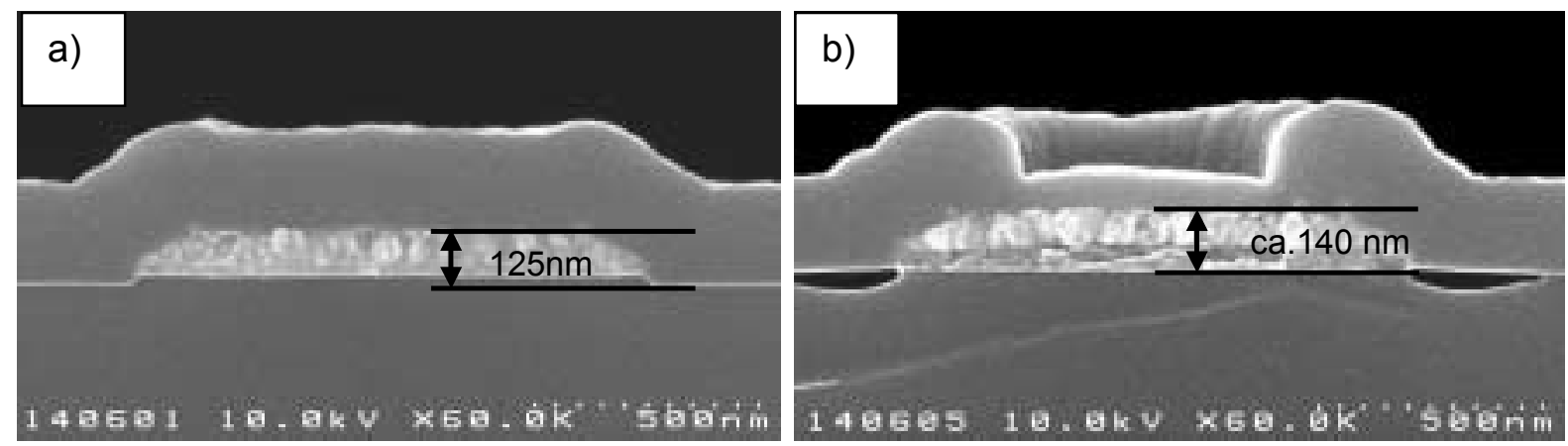

Abb. 7.6: a) Nicht oxidiertes TaN nach Sauerstofftemperung mit $\mathrm{Si}_{3} \mathrm{~N}_{4}-\mathrm{K}$ apselung während der Temperung, b) oxidiertes $\mathrm{TaN}$ nach Sauerstofftemperung ohne $\mathrm{Si}_{3} \mathrm{~N}_{4}$-Kapselung während der Temperung

In Abb. 7.6 a) ist das REM Bild des vollständig mit Siliziumnitrid eingekapselten Barrierenschichtstapel nach der Temperaturbehandlung gezeigt, das $\mathrm{IrO}_{2}$ und das $\mathrm{Ir}$ sind aufgrund der zu schwachen Vergrößerung in dieser Aufnahme als nicht trennbare helle Struktur zu erkennen, das darunter befindliche TaN ist grau. Der in Abb. 7.6 a) eingezeichnete Pfeil deutet die Dicke der drei Schichten an. Es ist zu keiner Dickenzunahme nach der thermischen Behandlung gekommen, die hier nicht gezeigte unstrukturierte Referenzprobe zeigt ebenfalls keine Oxidation der TaN Schicht. In Abb. 7.6 b) ist die gleiche Struktur zu sehen, es ist nach der Temperaturbehandlung mit geöffneter Siliziumnitridkapselung in Sauerstoffatmosphäre jedoch zu einer signifikanten Dickenzunahme der TaN Schicht gekommen. Die Dicke des $\mathrm{IrO}_{2} / \mathrm{Ir} / \mathrm{TaN}_{\mathrm{x}} \mathrm{O}_{\mathrm{y}}$ Stapels ist etwa $\mathrm{d}=140 \mathrm{~nm}$, über der $\mathrm{IrO}_{2}$ Schicht ist nach der Temperung ein Nitrid $(\mathrm{d}=90 \mathrm{~nm})$ abgeschieden worden, um ein Abplatzen der obersten Schichten bei der 
Präparation zu vermeiden und um die Schichtdicken ausmessen zu können. Die Kontraste im Siliziumoxid sind vermutlich auf Spannungseffekte aufgrund der Oxidation zurückzuführen.

Anhand der gezeigten Ergebnisse lässt sich schließen, dass der strukturierte Barrierenschichtstapel in Abwesenheit von Sauerstoff intakt und leitfähig bleibt, da kein Sauerstoff aus dem geätzten $\mathrm{IrO}_{2}$ in die unterliegenden Schichten gelangt. Die Tatsache, dass aus der geöffneten Struktur, bei der das Siliziumnitrid oberhalb des $\mathrm{IrO}_{2}$ partiell entfernt wurde, die TaN Schicht signifikant aufoxidiert ist, lässt sich nur über eine Sauerstoffdiffusion entlang der Grenzflächen erklären. Da nach den REM-Bildern die gesamte TaN Schicht gleichmäßig oxidiert ist, ist auch keine Leitfähigkeit dieser Schichtstruktur mehr zu erwarten.

\subsection{Integration mittels zweidimensionalem Konzept}

Aufgrund der Ergebnisse aus Kap. 7.2 stellt die dreidimensionale Integration ein komplexes Problem dar, das eventuell mit besseren Kapselschichten, die die Grenzflächendiffusion unterbinden, gelöst werden kann. Dies soll jedoch nicht weiter verfolgt werden, sondern es soll gezeigt werden, dass eine Integration der Barrierenschichten mit dem zweidimensionalen

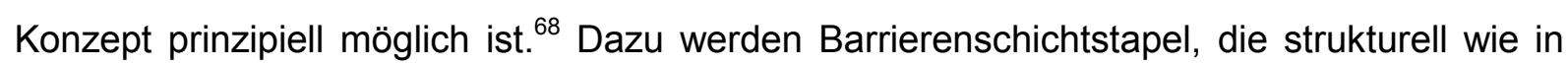
Kap. 7.1 beschrieben aufgebaut sind, nach der Hochtemperaturbehandlung bei $700^{\circ} \mathrm{C}$ mit einem sehr einfachen, stark anisotropen Ätzprozess auf eine laterale Strukturgröße von $\mathrm{d}=1,2 \mu \mathrm{m}$ strukturiert und anschließend mittels vorher erzeugten KontaktlochkettenStrukturen geprüft, ob der Barrierenschichtstapel einen für die Integration notwendigen niederohmigen elektrischen Kontakt darstellt [Mur93]. Bei den Kontaktlochketten sind jeweils zwei poly-Si-plugs abwechselnd über den strukturierten Barrierenschichtstapel und über ein hochdotiertes Si-Gebiet elektrisch verbunden. Die Kontaktlochkettenstruktur ist in Abb. 7.7 schematisch gezeigt. Um den Widerstand eines Barrierenschichtstapels mit Si-plug zu messen, wurde der Gesamtwiderstand der Kontaktlochkettenstrukturen mit Messspannungen von $1 \mathrm{~V}<\mathrm{U}_{\text {Mess }}<5 \mathrm{~V}$ evaluiert. Dazu wurden Kontaktlochketten mit jeweils 10,100 und 1000 in Serie geschaltete Kontakten gemessen und der Einzelwiderstand durch Division des Gesamtwiderstands durch die Anzahl der plugs ermittelt. Dabei ist als plug-Widerstand der Bahnwiderstand der Reihenschaltung eines dotierten Si-Gebiets, eines poly-Si plugs und des Barrierenschichtstapels zu verstehen. Der Barrierenschichtstapel selbst stellt wiederum eine Parallelschaltung der einzelnen Schichten dar, von denen zwei übereinanderliegende Schichten $\mathrm{i}$ und $\mathrm{j}$ den Kontaktwiderstand $\mathrm{R}_{\mathrm{K}, \mathrm{j}}$ besitzen. Aufgrund der einfachen Messanordnung ist es nicht möglich, die einzelnen Widerstände zu separieren, es soll mit der Bestim-

\footnotetext{
${ }^{68}$ Die Experimente sind in Zusammenarbeit mit Herrn Dr. W. Hartner (Infineon Technologies AG)
} durchgeführt worden. 
mung des Bahnwiderstands nur geprüft werden, ob der Barrierenschichtstapel einen ohmschen Kontakt darstellt.

\section{Messpunkt 1}

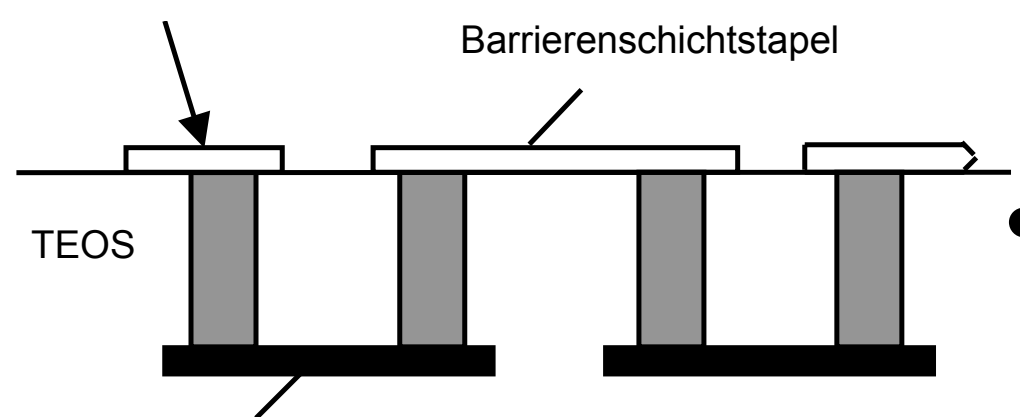

Hochdotiertes Si-Gebiet
Messpunkt 2

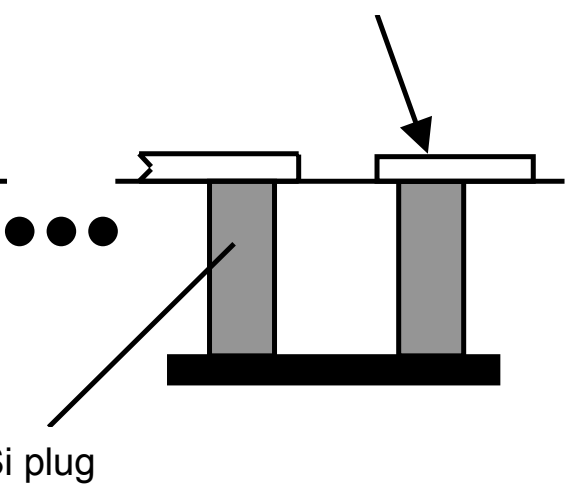

Abb. 7.7: Kontaktlochkettenstrukturen zur Bestimmung des Bahnwiderstandes

Dazu wurden Barrierenschichtstapel mit verschiedenen Metall-SiliziumInterdiffusionsbarrieren (TiN, TaN und TaSiN) hergestellt und verglichen. Die REM Bilder der strukturierten $\mathrm{Pt} / \mathrm{IrO}_{2} / \mathrm{Ir} / \mathrm{TiN}$-Barrierenschichtstapel sind in Abb. 7.8 gezeigt; aufgrund des nicht optimierten anisotropen Ätzprozesses ist zu erkennen, dass Redepositionen des abgesputterten Materials zu unsauberen Ätzkanten führen, teilweise ist ein Abplatzen der Pt Schicht an der Ätzkante zu sehen.
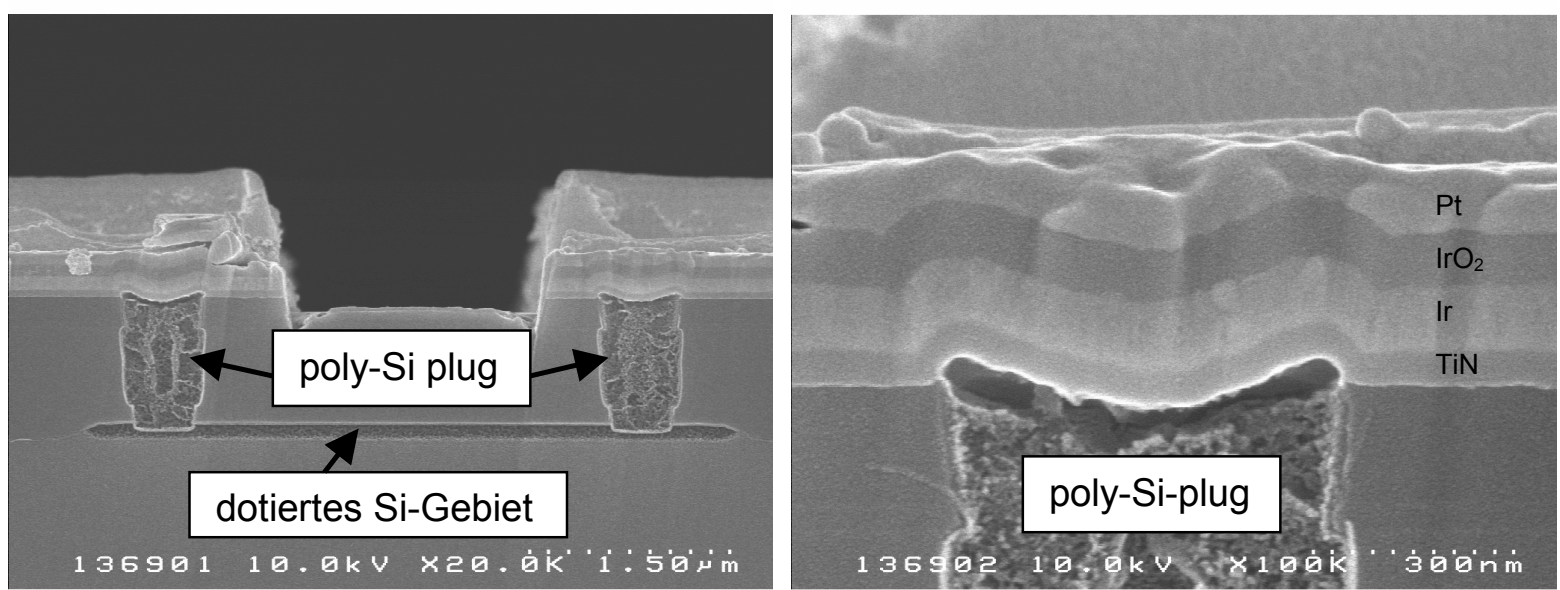

Abb. 7.8: REM-Analysen der Barrierenschichtstapel mit TiN Kontaktschicht nach der Hochtemperaturbehandlung und der anschließenden Strukturierung

Die Ergebnisse der Bahnwiderstände mit den statistisch am aussagekräftigsten 1000erKontaktlochketten sind in Abb. 7.9 gezeigt. Es konnte keine signifikante Abhängigkeit von der gewählten Spannung nachgewiesen werden, wie man es für einen ohmschen Kontakt 
erwartet. Die Gesamtwiderstände liegen, unabhängig von der Kettenlänge, für die Barrierenschichtstapel mit TiN und TaN als Kontaktschichten bei etwa 600-700 $\Omega /$ plug, für die TaSiN Schichtstapel bei $1650 \Omega /$ plug. Widerstandswerte in dieser Größenordnung stellen aufgrund des RC-Gliedes in einer Serienschaltung von Kondensator und einem ohmschen Widerstand keine Einschränkung der Schreibgeschwindigkeit einer ferroelektrischen Kondensatorzelle dar [Böt01].

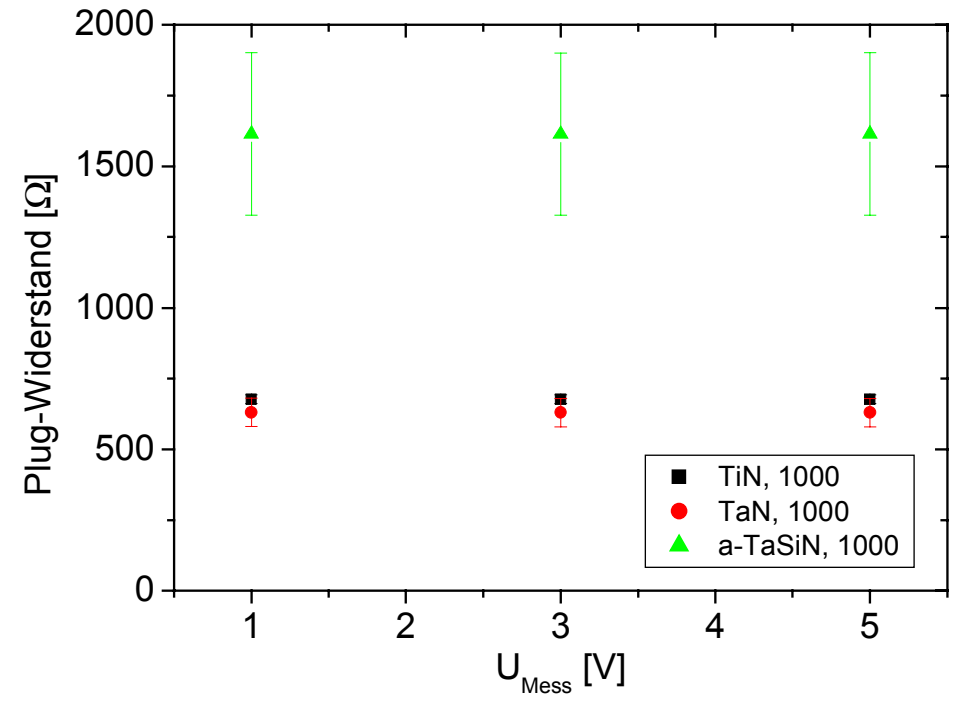

Abb. 7.9: Widerstände der Kontaktlochketten nach $700^{\circ} \mathrm{C}$ Sauerstoff-Auslagerung

Damit stellt die untersuchte, auf $\mathrm{IrO}_{2} / \mathrm{Ir}$ Schichten basierende Barrierenschichtabfolge, unabhängig von der gewählten Metall-Silizium-Interdiffusionsbarriere mit dem vorgestellten zweidimensionalen Konzept eine gangbare Lösung für die Integration der ferroelektrischen SBTund PZT Schichten in einen 4Mbit-Chip dar. 


\section{Zusammenfassung}

In dieser Arbeit wurden $\mathrm{IrO}_{\mathrm{x}}$ Sauerstoffdiffusionsbarrierenschichten mittels reaktivem Sputtern deponiert und eine Charakterisierung der Schichten vorgenommen. Dabei wurde zur Optimierung des Depositionsprozesses ein Modell entwickelt und angewendet, das den Sauerstoff-Getter-Effekt des kondensierenden Metalls während des reaktiven Depositionsprozesses beschreibt. Durch Maximierung des reaktiven Sauerstoffflusses $Q_{S}$ auf das Substrat ist es gelungen, aus den generischen Plasmakurven die für die Deposition von stöchiometrischen $\mathrm{IrO}_{2}$ Schichten nötigen Sputterparameter aufzufinden.

Diese so optimierten $\mathrm{IrO}_{2}$ Schichten weisen eine gute elektrische Leitfähigkeit auf, der spezifische Widerstand der Schichten liegt für eine Depositionstemperatur von $100^{\circ} \mathrm{C}$ bei $\rho_{\text {IrO }}=280 \mu \Omega \mathrm{cm}$, für die höchste untersuchte Depositionstemperatur von $500^{\circ} \mathrm{C}$ bei $\rho_{\text {IrO }}=125 \mu \Omega \mathrm{cm}$. Es wurde mittels Spannungsmessungen nachgewiesen, dass die feinkristallinen $\mathrm{IrO}_{2}$ Schichten aufgrund der zur Herstellung verwendeten Parameter hohe intrinsische, kompressive Spannungen von etwa $\sigma_{c}=-1,6 \mathrm{GPa}$ aufweisen, die mit einem lonPeening Modell gedeutet wurden. Dieses von Windischmann entwickelte Modell wurde in dieser Arbeit benutzt, um die mit der Röntgenbeugung gefundenen, systematisch temperaturabhängigen dilatativen Gitterstörungen im tetragonalen $\mathrm{IrO}_{2}$ Kristallgitter zu erklären. Die gefundenen Gitterdilatationen sind bereits bei den niedrigsten für die Deposition sinnvollen Sputterleistungen von $\mathrm{P}=300 \mathrm{~W}$ signifikant und lassen sich auf die im Verhältnis zum TargetSubstrat-Abstand große freie Weglänge der Sauerstoffionen zurückführen. Die Energie dieser lonen übersteigt bei der reaktiven Sputter-Deposition deutlich die Wigner-Energie der Sauerstoffatome in der $\mathrm{IrO}_{2}$ Schicht, was zu einer Erzeugung einer hohen Defektdichte in der Schicht führt. Anhand von Querschliff-Transmissionselektronenmikroskopie-Analysen konnte an den $\mathrm{IrO}_{2}$ Schichten eine für die gewählten Depositionsparameter typische Zone-T Mikrostruktur nachgewiesen werden [Tho74], [Tho75].

Mit den Analysen zum Rekristallisationsverhalten konnte gezeigt werden, dass stark sauerstoffdefizitäre $\mathrm{IrO}_{2-x}$ und bereits leicht sauerstoffüberstöchiometrisch deponierte $\operatorname{IrO}_{2+x}$ Schichten beim Anlassen einer starken Degradation unterliegen, welche die Schichten als Barrieren unbrauchbar macht. Dieses Verhalten ist auf eine Instabilität der stark übersättigten Ir und $\mathrm{IrO}_{2}$ Phase zurückgeführt worden. Nahezu stöchiometrisch gesputterte $\mathrm{IrO}_{2}$ Schichten weisen ein Rekristallisationsverhalten auf, das stark vom mikrostrukturellen Deformationsgrad der Schicht abhängig ist. Dieser Deformationsgrad wird aber gerade durch den im Sauerstoffplasma stattfindenden lon-Peening-Prozess und durch die zur Abscheidung verwendete Substrattemperatur bestimmt: Durch hohe Substrattemperaturen thermisch angeregte Relaxationsprozesse begünstigen das partielle Ausheilen von Defekten, während 
eine niedrige Substrattemperatur das Einfrieren einer hohen Defektdichte und damit eine deutlich stärkere dilatative Gitterdeformation von 3-4\% bei den untersuchten Depositionsleistungen mit sich bringt. Als Konsequenz dieser sehr großen Deformation gibt es beim Anlassen der kaltgesputterten Proben über eine Temperatur von $400^{\circ} \mathrm{C}$ eine treibende $\mathrm{Kraft}$ für einen Rekristallisationsprozess, bei dem die geschädigten $\mathrm{IrO}_{2}$ Körner graduell durch das Heranwachsen einer neuen Generation von relaxierten Körnern ersetzt werden. Für die heißgesputterten $\mathrm{IrO}_{2}$ Schichten ist aufgrund der geringeren kristallinen Deformation kein solcher Rekristallisationsprozess nachweisbar, sondern es findet bis zu einer kritischen Temperatur von etwa $750^{\circ} \mathrm{C}$ nur ein kontinuierliches Ausheilen der Defekte statt.

Anhand der in dieser Arbeit erstmals an $\mathrm{IrO}_{2}$ Schichten gemessenen Sauerstofftracerdiffusion konnte gezeigt werden, dass fast vollständig relaxierte Schichten eine sehr niedrige intrinsische Sauerstoffmobilität aufweisen. Die gefundene Temperaturabhängigkeit der Diffusion kann mit einem Arrhenius Gesetz beschrieben werden und lässt sich aufgrund der errechneten Aktivierungsenergie von $E_{A}=(2,73 \pm 0,07) \mathrm{eV}$ und geometrischer Überlegungen zum Rutil-Gittertyp durch einen Leerstellenmechanismus deuten, der ebenfalls in isostrukturellen tetragonalen Systemen wie $\mathrm{TiO}_{2}$ als dominierender Mechanismus gefunden wurde [Hau65].

Es konnte weiterhin an $\mathrm{IrO}_{2}$ Schichten mit residualer Gitterdilatation von über $1 \%$ eine signifikant erhöhte Sauerstofftracerdiffusion nachgewiesen werden. Bereits bei relativ geringen Gitterdilatationen lässt sich eine Erhöhung von ein bis drei Größenordnungen im Diffusionskoeffizienten messen, so dass nur rekristallisierte $\mathrm{IrO}_{2}$ Proben mit geringen Defektdichten den an die Barrierenschicht gestellten, hohen Integrationsansprüchen gerecht werden. Für die Integration der ferroelektrischen SBT und PZT Schichten sind einerseits lange Kristallisations- und Rekristallisationsschritte bei typischerweise $700^{\circ} \mathrm{C}$ notwendig, andererseits ist die Schichtdicke der Sauerstoffbarriere aus Integrationsgründen nicht zu beliebigen Dicken skalierbar. Dies ist durch die für die Strukturierung notwendigen Ätzprozesse begründet, die bei großen Schichtdicken zu einer Strukturaufweitung führt, außerdem können starke Schichtspannungen zum Adhäsionsverlust und damit leicht zum Ablösen von dicken Schichten führen. Überdies konnte durch SIMS-Analysen von Sauerstoff-Diffusionsprofilen in Ir Schichten gezeigt werden, dass Ir Schichten nicht als Sauerstoffbarriere für die erforderlichen hohen Anlass-Temperaturen geeignet sind.

In dieser Arbeit konnte erstmals gezeigt und belegt werden, dass durch Verwenden von optimierten $\mathrm{IrO}_{2}$ Schichten eine erfolgreiche Integration mit einem Konzept realisierbar ist, das die zusätzlich auftretende parasitäre Diffusion entlang von geätzten Grenzflächen ausschließt. Durch das nachträgliche Ätzen des Barrierenschichtstapels nach der Hochtemperaturbehandlung ist es möglich, eine ausreichend niederohmige elektrische Kontaktierung des 
ferroelektrischen Kondensators mit dem CMOS-Transistor herzustellen. Dabei wurden Schichtdicken von jeweils $\mathrm{d}=100 \mathrm{~nm}$ für die $\mathrm{IrO}_{2}$ Sauerstoffbarriere, für die Ir Schicht und die auf einem Nitrid basierende Silizium/Metall-Interdiffusionsbarriere verwendet, die für die Integration in einen 4Mbit-Chip eine realisierbare Gesamtdicke des Barrierenschichtstapels ergeben. Prinzipiell ist aber auch durch Dickenoptimierung der Einzelschichten Potential für die Verwendung des diskutierten Schichtstapels für deutlich höhere Integrationsdichten gegeben.

Aufgrund der in dieser Arbeit gefundenen Ergebnissen zur Abhängigkeit des Rekristallisationsprozesses in den gesputterten $\mathrm{IrO}_{2}$ Schichten konnte gezeigt werden, dass ein genaues Verständnis des Einflusses der Depositionsparameter für die erfolgreiche Integration der Schichten unerlässlich ist. Es kann weiterhin gefolgert werden, dass eine Deposition einer defektarmen $\mathrm{IrO}_{2}$ Schicht für die Anwendung als Barrierenschicht als Vorteil angesehen werden kann. Der diskutierte lonenbeschuss während der reaktiven Sputterabscheidung ließe sich durch das für Oxidschichten (wie beispielsweise Hochtemperatursupraleiter) verwendete Hochdrucksputtern oder das Benutzen einer Off-Axis-Geometrie vermeiden, da dadurch ein direktes lonen-Bombardement der aufwachsenden Schicht reduziert wird [Pop95].

Durch die fortschreitende Optimierung der MOD und MOCVD Depositionsverfahren und der Ausgangs-Materialien bzw. Zusammensetzung der ferroelektrischen Schichten scheint es möglich, die notwendigen Kristallisationstemperaturen auf deutlich unter $700^{\circ} \mathrm{C}$ senken zu können. Unter diesen Umständen wäre es denkbar, auf die Optimierung der hochtemperaturstabilen $\mathrm{IrO}_{2}$ Barrierenschichten zu verzichten und auf andere, weniger aufwändige und kostengünstiger abzuscheidende Materialien auszuweichen. Nach dem derzeitigen Stand der Forschung unterdrückt allerdings die Präsenz einer oxidischen Elektrode, insbesondere die des $\mathrm{IrO}_{2}$, die unerwünschte, elektrische Langzeitdegradation der Ferroelektrika, die sich in einem Verlust der effektiven Polarisation mit zunehmender Anzahl von Schreib- und Lesevorgängen äußert [Nak94], [Lee99]. Das zuverlässige Auslesen der Zellinformation nach $10^{12}-10^{15}$ Schreib-/Lesezyklen stellt aber eine der Grundvoraussetzungen für die Anwendungen dieser ferroelektrischen Materialien in den angestrebten Speicherprodukten dar. In dieser Hinsicht verbinden nur optimierte $\mathrm{IrO}_{2}$ Schichten sehr gute Elektroden- mit exzellenten Diffusionsbarriereneigenschaften und sind daher auch in Zukunft als das vielversprechendste Material für hochintegrierte Speicherapplikationen anzusehen, die auf oxidischen $\mathrm{Pe}-$ rowskit-Ferroelektrika basieren. 


\section{Literaturverzeichnis}

[Adl68] D. Adler, Rev. Mod. Phys. 40(4), 714-736 (1968)

[Age72] V.N. Ageev, N.I. Ionov, Sov. Phys. Techn. Phys. 16(10), 1742 (1972)

[Ale97] H. Alexander, Physikalische Grundlagen der Elektronenmikroskopie, Stuttgart, Teubner (1997)

[All93] C.E. Allevato, C.B. Vining, J. of Alloys and Compounds 200, 99 (1993)

[Ans98] D'Ans Lax, Elemente, anorganische Verbindungen und Materialien, Minerale, Band 3, 4. Auflage, Herausg. R. Blachnik, Berlin, Springer Verlag, 497, 768 (1998)

[Aok98] K. Aoki, T. Sakoda, Y. Fukuda, Jpn. J. Appl. Phys. 37, 522 (1998)

[Ara95] C.A-Paz de Araujo, J.D. Cuchiaro, L.D. McMillan, M.C. Scott, J.F. Scott, Nature (London) 374, 627 (1995)

[Auc98] O. Auciello, J.F. Scott, R. Ramesh, The Physics of Ferroelectric Memories, Physics Today 51, 22-27, (1998)

[Bal99] J. Baliga, Semiconductor International 11/99, 79-90 (1999)

[Beb88] X. Beebe, T.L. Rose, IEEE Trans. Biomed. Engin. 35, 494 (1988)

[Beh91] R. Behrisch, K. Wittmaack, (Herausg.), Topics in Applied Physics, Vol. 64, Sputtering by Particle Bombardment III, Springer Verlag, Berlin, Heidelberg, 1ff. (1991)

[Bei99] G. Beitel, H. Wendt, E. Fritsch, V. Weinrich, M. Engelhardt, B. Hasler, T. Röhr, R. Bergmann, U. Scheler, K.-H. Malek, N. Nagel, A. Gschwandtner, W. Pamler, W. Hönlein, C. Dehm, C. Mazuré, Microelectr. Engineer. 48, 299-302 (1999)

[Ben83] G. Beni, J.L. Shay, Phys. Rev. B 21, 364 (1983)

[Ber76] R.S. Berg, G.J. Kominiak, J. Vac. Sci. Technol. 13(1), 403 (1976)

[Ber87] S. Berg, H.-O. Blom, T. Larsson, C. Nender, J. Vac. Sci. Technol. A 5(2), 202-207 (1987)

[Biè93] P. de Bièvre, P.D.P. Taylor, Int. J. Mass Spectrom. Ion Processes 123, 149-166 (1993)

[Bis89] H.E. Bishop, Methods Of Surface Analysis, Editor J.M. Walls, Cambridge University Press (1989) 
[Böt01] U. Böttger, Neue Materialien für die Informationstechnik, Vorlesungsmanuskript C2, 32. IFF-Ferienkurs Band 7, (2001)

[Bro99] U. Brossmann, R. Würschum, U. Södervall, H.-E. Schaefer, J. Appl. Phys. 85(11), 7646 (1999)

[Cab00] C. Cabral, Jr., K.L. Saenger, D.E. Kotecki, J.M.E. Harper, J. Mater. Res. 15(1), 194$198(2000)$

[Cha00] B.R. Chalamala, Y. Wei, R.H. Reuss, S. Aggarwal, S.R. Perusse, B.E. Gnade, R. Ramesh, J. Vac. Sci. Technol. B 18, 1919 (2000)

[Cha99] S.Y. Cha, H.C. Lee, Jpn. J. Appl. Phys. 38, 1128-1130 (1999)

[Cho01] S.K. Choi, J.I. Lee, J. Vac. Sci. Technol. A 19(5), 2043 (2001)

[Cho71] A. Chow, Talanta 19, 899-902, Pergamon Press, Northern Ireland (1971)

[Cho95] H.M. Choi, S.K. Choi, J. Vac. Sci. Technol. A 13(6), 2832 (1995)

[Cho97] H.-J. Cho, H. Horii, C.S. Hwang, J.-W. Kim, C.S. Kang, B.T. Lee, S.I. Lee, Y.B. Koh, M.Y. Lee, Jpn. J. Appl. Phys., Part 136, 1722-1727 (1997)

[Chu78] W.K. Chu, J.W. Mayer, M.-A. Nicolet, Backscattering Spectrometry, Academic Press (1978)

[Chu96] Y.-C. Chung, B. Wuensch, J. Appl. Phys. 79(11), 8323 (1996)

[Cla63] A.D. Le Claire, Brit. J. Appl. Phys. 14, 351 (1963)

[Cor62] E.H.P. Cordfunke, G. Meyer, Rec. Trav. Chim. 81, 495-504, 670-678 (1962)

[Cot67] A.H. Cottrell, An Introduction to Metallurgy, Christ's College Cambridge, Edward Arnold (Publishers) Ltd., London (1967)

[Cox89] D.F. Cox, T.B. Fryberger, S. Semancik, Surf. Sci. 224, 121 (1989)

[Cra67] J. Crank, Mathematics of diffusion, Clarendon Press (1967)

[Cri85] R. v.Criegern, I. Weitzel, H. Rehme, Analyse der Tiefenverteilung von Dotierstoffen mit der Sekundär-Massenspektrometrie (SIMS), Siemens Forsch.- u. Entwickl.-Ber. Bd.14(4) 208- 215 (1985)

[Cul78] B.D. Cullity, Elements of x-ray diffraction, 2nd edition, Addison-Wesley, Reading, 84 (1978)

[Dan84] R.R. Daniels, G. Margaritondo, C.-A. Georg, F. Lévy, Phys. Rev. B 29(4) 1813-1818 (1984)

[Dar92] R. Darji, Interner Forschungsbericht, Siemens AG, München, ZFE BT MR 74 (1992) 
[Deh99] C. Dehm, W. Hartner, G. Schindler, R. Bergmann, B. Hasler, I. Kasko, M. Kastner, M. Schiele, V. Weinrich, C. Mazuré, Integr. Ferroelec. 26, 197-213 (1999)

[Dem99] V. Demuth, H.P. Strunk, D. Wörle, C. Kumpf, E: Burkel, M. Schulz, Appl. Phys. A 68, 451 (1999)

[Des99] S.B. Desu, D.P. Vijay, S. Ramanathan, H.D. Bhatt, S. Tirumala, Thin Solid Films 350, 21-29 (1999)

[Dev49] A.F. Devonshire, Phil. Mag. 740, 1040 (1949)

[Doo85] L.R. Doolittle, Nucl. Instr. Meth. B9, 344 (1985)

[EIl65] R.P. Elliott, Constitution of Binary Alloys, First Supplement, Mc Graw-Hill, New York (1965)

[Eva88] J.T. Evans, R. Womack. An experimental 512-bit nonvolatile memory with ferroelectric storage cell. IEEE Journal of Solid-State Circuits 23(5), 1171-1175 (1988)

[For68] M.A. Fortes, B. Ralph, Proc. Roy. Soc. [London] A 307, 431 (1968)

[Fra94] G. Franz, Oberflächentechnologie mit Niederdruckplasmen, 2. Aufl., Springer Verlag, Berlin, 11ff. (1994)

[Gal69] F.S. Galasso, Structure, properties and preparation of perovskite-type compounds, Pergamon Press, (1969)

[Gas99] S.M. Gasser, E. Kolawa, M.-A. Nicolet, J. Appl. Phys. 86(4), 1974 (1999)

[Gas99a] S.M. Gasser, R. Ruiz, E. Kolawa, M.-A. Nicolet, J. Electrochem. Soc. 146(4), 15461548 (1999)

[Ger97] C. Gerthsen, Gerthsen Physik, Springer Verlag, Berlin, 19. Aufl., 226 (1997)

[Gey92] U. Geyer, Rastertunnelmikroskopie an $\mathrm{YBa}_{2} \mathrm{Cu}_{2} \mathrm{O}_{7}$-Schichten: Interpretation der Bilder und der spektroskopischen Ergebnisse, Dissertation, Göttingen (1992)

[Gme78] Gmelin, Handbuch der Anorganischen Chemie, Iridium, Suppl. Vol.2, Springer, Berlin, 3ff. (1978)

[Goo71] J.B. Goodenough, in Progress in Solid State Chemistry, Vol.5, Herausg. H. Reiss, Pergamon Press, Ltd., Oxford, England (1971)

[Gra76] J.E. Graebner, E.S. Greiner, W.D. Ryden, Phys. Rev. B 13(6), 2426-2432 (1976)

[Gre88] N.N. Greenwood, A. Earnshaw, Chemie der Elemente, 1. Aufl., Weinheim, Basel, VCH-Verlag, 1430f. (1988)

[Gro52] W.R. Grove, Phil. Trans. Roy. Soc. London 142, 87 (1852) 
[Gro53] W.R. Grove, Philos, Mag. 5, 203 (1853)

[Haa94] P. Haasen, Physikalische Metallkunde, 3.Aufl., Springer, Berlin, 155 (1994)

[Har78] J.M.E. Harper, J.J. Cuomo, R.J. Gambino, H.R. Kaufman, R.S. Robinson, J. Vac. Sci. Technol. 15(4), 1597 (1978)

[Har97] T. Hara, M. Tanaka, K. Sakiyama, S. Onishi, K. Ishihara, J.Kudo, Jpn. J. Appl. Phys., Part 2 36, 893 (1997)

[Hau65] R. Haul, G. Dümbgen, J. Phys. Chem. Solids 26, Pergamon Press, 1-10 (1965)

[Hec81] M. Hecq, A. Hecq, J. Vac. Sci. Technol. 18(2), 219 (1981)

[Hof82] D.W. Hoffman, J.A. Thornton, J. Vac. Sci. Technol. 20(3), 355 (1982)

[Höf91] H.J. Höfler, H. Hahn, R.S. Averback, Defect and Diffusion Forum 75, 195 (1991)

[Hoo01] S. HoOn OH, H.M. JANG, PhYS. ReV. B 63, 132101 (2001)

[Hor95] E. Hornbogen, H. Warlimont, Metallkunde: Aufbau und Eigenschaften von Metallen und Legierungen, 3. Aufl., Springer Verlag, Berlin, 310 (1995)

[Hor99] R.H. Horng, D.S. Wuu, L.H. Wu, M.K. Lee, Superficies y Vacio 9, 139-142 (1999)

[Hud77] W.R. Hudson, J.Vac. Sci. Technol. 14(1), 286 (1977)

[Jaf71] B. Jaffe, W.R. Cook, Jr., H. Jaffe, Piezoelectric Ceramics, Academic Press, London (1971)

[JCP95] JCPDS-International Centre for Diffraction Data, Datenblätter 15-0870, 06-0598, 431019, 12 Campus Blvd., Newtown Square, PA 19073-3273 USA

[Jeo97] Y.-C. Jeon, J.-M.Seon, J.-H. Joo, K.-Y. Oh, J.-S. Roh, J.-J. Kim, D.-S. Kim, Appl. Phys. Lett. 71(4), 467 (1997)

[Jim91] M.C. Jimenez, M. Fernandez, J.M. Albella, J.M. Martinez-Duart, J. Vac. Sci. Technol. B 9(3), 1492 (1991)

[Joh81] N. M. Johnson, D. K. Biegelsen, M. D. Moyer, V. R. Deline, and C. A. Evans, Jr., Appl. Phys. Lett. 38, 995 (1981)

[Jon62] F. Jona, G. Shirane, Ferroelectric Crystals, International Series of Monographs on Solid State Physics, Editors R. Smoluchowski, N. Kurti, Pergamon Press, Oxford (1962)

[Kac99] T. Kacsich, S. Gasser, Y. Tsuji, A. Dommann, M.-A. Nicolet, J. Appl. Phys. 85, 1871 (1999)

[Kag98] Y. Kaga, Y. Abe, H. Yanagisawa, K. Sasaki, J. Appl. Phys. 37, 3457-3461 (1998)

[Kat82] T. Katsube, I. Lauks, J.N. Zemel, Sensors and Actuators 2, 399 (1982) 
[Kie31] H. Kiessig, Annalen d. Physik, 10(7), 769-788 (1931)

[Kim01] Sam-Dong Kim, In-Seok Hwang, Jin-Koo Rhee, Tae-Ho Cha, Heon-Do Kim, Electrochem. Solid-State Lett. 4, G7 (2001)

[Kim98] M.-H. Kim, S.-B. Baek, U. Paik, S. Nam, J.-D. Byun, J. Kor. Phys. Soc. 32, 1127$1130(1998)$

[Kin76] W. Kingery, H.K. Bowen, and D.R. UhImann, Introduction to Ceramics, $2^{\text {nd }}$ ed., Wiley, New York, 240-249 (1976)

[Kit96] C. Kittel, Einführung in die Festkörperphysik, 11. Aufl., Oldenbourg, München, 431ff. (1996)

[Kof72] P. Kofstad, Nonstoichiometry, Diffusion, and Electrical Conductivity in Binary Metal Oxides, Kap.5, Wiley-Interscience, New York, 69-95 (1972)

[Koh01] H. Kohlstedt, Neue Materialien für die Informationstechnik, Vorlesungsmanuskript D2, 32. IFF-Ferienkurs Band 7 (2001)

[Kol87] E. Kolawa, F.C.T. So, E.T.-S. Pan, M.-A. Nicolet, Appl. Phys. Lett. 50(13), 854-855 (1987)

[Kre86] K. Kreider, J. Vac. Sci. Technol. A 4, 606 (1986)

[Kri63] C.A. Krier, R.I. Jaffee, J. Less-Common Metals 5, 411-431 (1963)

[Kru83] L. Krusin-Elbaum, M. Wittmer, C.Y. Ting, J.J. Cuomo, Thin Solid Films 104, 81 (1983)

[Kru88] L. Krusin-Elbaum, M. Wittmer, J. Electrochem. Soc. 135(10), 2610-2614 (1988)

[Kru89] L. Krusin-Elbaum, Thin Solid Films 169, 17-24 (1989)

[Kub67] O. Kubaschewski, E.L.L. Evans, C.B. Alcock, Metallurgical Thermochemistry, $4^{\text {th }}$ edition, Pergamon, London (1967)

[Kur97] R. Kurt, W. Pitschke, A. Heinrich, J. Schumann, J. Thomas, K. Wetzig, A. Burkov, Thin Solid Films 310, 8-18 (1997)

[Kus90] E. Kusano, D.M. Goulart, Thin Solid Films 193/194, 84-91 (1990)

[Lan37] L.D. Landau, Phys. Z. Sowjetunion 11, 26 (1937)

[Lee95] J.J. Lee, C.L. Thio, S.B. Desu, J. Appl. Phys. 78, 5073 (1995)

[Lee96] J.S. Lee, H.J. Kwon, Y.W. Jeong, H.H. Kim, C.Y. Kim, J. Mater. Res. 11(11), 26812684 (1996) 
[Lee99] H.-S. Lee, W.-S. Um, K.-W. Hwang, H.-G. Shin, Y.-B. Kim, K.-H. Auh, J. Vac. Sci. Technol. A 17(5), 2939 (1999)

[Leg94] P.B. Legrand, J.P. Dauchot, M. Hecq, M. Charbonnier, M. Romand, J. Vac. Sci. Technol. A 12(4), 1551 (1994)

[Li00] A. Li, D. Wu, H. Ling, T. Yu, M. Wang, X. Yin, Z. Liu, N. Ming, J. Appl. Phys. 88, 1035 (2000)

[Lia98] P.C. Liao, W.S. Ho, Y.S. Huang, K.K. Tiong, J. Mater. Res. 13, 1318-1326 (1998)

[Lin00] J. Lin, K. Natori, Y. Fukuzumi, M. Izuha, K. Tsunoda, K. Eguchi, K. Hieda, D. Matsunaga, Appl. Phys. Lett. 76(17), 2430-2432 (2000)

[Lun78] T. S. Lundy and W. A. Coughlan, Diffusion and Defect Data, ed. by F. H. Wöhlbier, Trans. Tech. Publ. 16, Aedermannsdorf, Switzerland, 169 (1978)

[Luo01] J. Appl. Phys. 89, 6760 (2001)

[Mae95] K. Maex, M. v. Rossum, (Edit.), Properties of Metal Silicides, Inspec, London, 10 (1995)

[Mas86] T.B. Massalski, Binary Alloy Phase Diagrams, Vol.2, Ed. W.W. Scott, Jr., American Society for Metals, Ohio (1986)

[Mat00] S. Matsushita, M. Harada, T. Gueshi, Y. Matsushita, Appl. Phys. Lett. 77(20), 3200 (2000)

[Mat00] Y. Matsui, M. Hiratani, S. Kimura, Jpn. J. Appl. Phys. 39, 256-263 (2000)

[Mat76] L.F. Mattheiss, Phys. Rev. B 13(6), 2433-2450 (1976)

[May70] A.F. Mayadas, M. Shatzkes, Phys. Rev. B 15, 1382 (1970)

[Mcl90] Mclntyre, J.E. Greene, G. Hakansson, J.-E. Sundgren, W.-D. Münz, J. Appl. Phys. 67(3), 1542-1553 (1990)

[Mil92] S.L. Miller, P.J. McWhorter, J. Appl. Phys.72, 5999 (1992)

[Moo01] B.K. Moon, K. Hironaka, C. Isobe, S. Hishikawa, J. Appl. Phys. 89(11), 6370 (2001)

[Mot61] N.F. Mott, Phil. Mag. 6, 287 (1961)

[Mur93] S. Murarka, Metallization: Theory and Practice for VLSI and ULSI, Boston, Butterworth-Heinemann (1993)

[Mur94] P.W. Murray, F.M. Leibsle, C.A. Muryn, H.J.Fischer, C.F.J. Flipse, G. Thornton, Phys. Rev. Lett. 72(5), 689 (1994) 
[Nag00] N. Nagel, T. Mikolajick, I. Kasko, W. Hartner, M. Mört, C.U. Pinnow, C. Dehm , C. Mazuré, An overview of FeRAM technology for high density applications, MRS Fall Meeting, Boston, USA (2000)

[Nag01] N. Nagel, private Mitteilungen (2001)

[Nag99] B. Nagaraj, T. Sawhney, S. Perusse, S. Aggarwal, V.S. Kaushik, S. Zafar, R.E. Jones, J.H. Lee, V. Balu, J. Lee, Appl. Phys. Lett. 74(21), 3194 (1999)

[Nak94] T. Nakamura, Y. Nakao, A. Kamisawa, H. Takasu, Jpn. J. Appl. Phys. 33, 5207$5210(1994)$

[Nak94a] T. Nakamura, Y. Nakao, A. Kamisawa, H. Takasu, Appl. Phys. Lett. 65(12), 15221524 (1994)

[Nev80] L. Nevot, P. Croce, Rev. Phys. App. 15, 761-769 (1980)

[Nis97] K. Nishio, T. Sei, T. Tsuchiya, SPIE 3136, 419 (1997)

[Nog96] T. Noguchi, T. Hase, Y. Miyasaka, Jpn. J. Appl. Phys. 35, 4900-4904 (1996)

[Oh00] S.H. Oh, C.G. Park, C. Park, Thin Solid Films 359, 118-123 (2000)

[Oka86] A. Okamoto, T. Serikawa, Thin Solid Films 137, 143-151 (1986)

[Pam01a] W. Pamler, private Mitteilungen

[Pam01] W. Pamler, Z. Gabric, Advances in Single Wafer Chemical Vapor Deposition of Oxide and Nitride Films, $12-6^{\text {th }}$ Int. Sympos. On Silicon Nitride and Silicon Dioxide Thin Insulating Films, Washington, DC (2001)

[Pan98] B. Panda, A. Dhar, G.D. Nigam, D. Bhattacharya, S.K. Ray, J. Appl. Phys. 83(2), 1114 (1998)

[Par54] L.G. Parratt, Physical Review 5(2), 359-369 (1954)

[Pet79] S. Petersson, J. Baglin, W. Hammer, F. D’Heurle, T.S. Kuan, I. Ohdomari, J. de Sousa Pires, P. Tove, J. Appl. Phys. 50(5), 3357 (1979)

[Peu84] M. Peuckert, Surf. Sci. 144, 451-464 (1984)

[Pop95] U. Poppe, 26. IFF-Ferienkurs, Elektrokeramische Materialien: Grundlagen und Anwendungen, Vorlesungs-Manuskript B4, (1995)

[Pow99] R.A. Powell, S.M. Rossnagel, Thin Films, PVD for Microelectronics: Sputter Deposition Applied to Semiconductor Manufacturing, Vol.26, Academic Press, San Diego (1999)

[Ram01] www.ramtron.com, Ramtron Intl Corp, 1850 Ramtron Drive, Colorado Springs, CO 80921 USA (2001) 
[Rao74] C.N. Rao, G.V. Subba Rao, National Bureau of Standards Report No. NSRDS-NBS49 (1974)

[Ras82] G. Rasigny, F. Varnier, M. Rasigni, J.P. Palmari, A. Llebaria, Phys. Rev. B 25(4), 2315 (1982)

[Ras83] G. Rasigny, F. Varnier, M. Rasigni, J.P. Palmari, A. Llebaria, Phys. Rev. B 27(2), 819 (1983)

[Ras84] G. Rasigny, F. Varnier, M. Rasigni, A. Llebaria, Phys. Rev. B 29(10), 5931 (1984)

[Rei95] J.S. Reid, Amorphous ternary diffusion barriers for silicon metallizations, Ph.D. Thesis, Pasadena, California, USA (1995)

[Ren94] G. Renaud, B. Villette, I. Vilfan, A. Bourret, Phys. Rev. Lett. 73, 1825 (1994)

[Rob83] L.S. Robblee, J.L. Lefko, S.B. Brummer, J. Electrochem. Soc. 130, 731 (1982)

[Rob86] L.S. Robblee, M.M. Mangaudis, E.D. Lasinsky, A.G. Kimball, S.B. Brummer, Mat. Res. Soc. Symp. 55, 303 (1986)

[Röm97] Römpp Lexikon, Chemie Band 3, 10. Aufl., Her. J. Falbe und M. Regitz, Georg Thieme Verlag, Stuttgart, 1986 (1997)

[Ros89] S.M. Rossnagel, J.J. Cuomo, W.D. Westwood, Handbook of Plasma Processing Technology, Noyes, Park Ridge, 233 (1989)

[Ros98] S.M. Rossnagel, J. Vac. Sci. Technol. B 16, 2585 (1998)

[Ryd70] W.D. Ryden, A.W. Lawson, C.C. Sartain, Phys. Rev. B 1(4), 1494-1500 (1970)

[Ryd72] W.D. Ryden, W.A. Reed, E.S. Greiner, Phys. Rev. B 6(6), 2089-2093 (1972)

[Sae01] K.L. Saenger and D.A. Neumayer, J. Appl. Phys. 89(6), 3132-3137 (2001)

[Sae98] K.L. Saenger, A. Grill, C. Cabral, Jr., J. Mater. Res. 13, 462 (1998)

[Sak99] T. Sakoda, K. Aoki, Y. Fukuda, Jpn. J. Appl. Phys. 38, 3600 (1999)

[Sam82] G.V. Samsonov, The Oxide Handbook, 2nd edition, Plenum, New York (1982)

[Sch01] H. Schroeder, Vorlesungsmanuskript C1, Neue Materialien für die Informationstechnik, 32. IFF Ferienkurs (2001)

[Sch60] H. Schäfer, H.J. Heitland, Z. Anorg. Allgem. Chem. 304, 249-265 (1960)

[Sch94] J. Schumann, D. Elefant, C. Glaudun, A. Heinrich, W. Pitschke, H. Lange, W. Henrion, R. Grötzschel, Phys. Stat. Sol. (a) 145, 429 (1994)

[Sco00] J.F. Scott, Ferroelectric Memories, Springer, Berlin (2000)

[Sie99] R. Sietmann, c't 6/99, 146-151 (1999) 
[Sig81] P. Sigmund, in R. Behrisch (Herausg.), Topics in Applied Physics, Vol. 47, Sputtering by Particle Bombardment I, Springer Verlag, Berlin, Heidelberg 65 (1981)

[Smi58] F.M. Smits, Measurement of Sheet Resistivities with the Four-Point Probe, Bell System Technical Journal, 711 (1958)

[Smi95] D.A. Smith, Thin Film Deposition: Principles and Practice, McGraw-Hill, New York, (1995)

[Smo61] G.A. Smolenskii, V.A. Isupov and A.I. Agranovskaya, Ferroelectrics of the oxygenoctahedral type with layered structure, Sov. Phys. Solid State 3, 651-655 (1961)

[Son00] Y.J. Song, H.H. Kim, S.Y. Lee, D.J. Jung, B.J. Koo, J.K. Lee, Y.S. Park, H.J. Cho, S.O. Park, K. Kim, Appl. Phys. Lett. 76(4), 451 (2000)

[Sto09] G.G. Stoney, Proc. R. Soc., London A 82, 172 (1909)

[Sub62] E.C. Subbarao, A family of ferroelectric bismuth compounds, J. Phys. Chem. Solids 23, 665-676 (1962)

[Suz64] T. Suzuoka, J. Phys. Soc. Jap. 19(6), 839 (1964)

[Syk89] D.E. Sykes, Methods Of Surface Analysis, Cambridge University Press (1989)

[Ter83] J. Tersoff, D.R. Hamann, Theory and Application for the scanning tunneling microscope, Physical Review Letters 50, 1998-2001 (1983)

[Ter85] J. Tersoff, D.R. Hamann, Theory of the scanning tunneling microscope, Physical Review B 31, 805-813 (1985)

[Tho74] J.A. Thornton, J. Vac. Sci. Technol. 11, 666 (1974)

[Tho75] J.A. Thornton J. Vac. Sci. Technol. 12, 830 (1975)

[Tho81] J.A. Thornton, D.W. Hoffman, J. Vac. Sci. Technol. 18(2), 203 (1981)

[Tho85] J.A. Thornton, D.W. Hoffman, J. Vac. Sci. Technol. A 3(3), 576 (1985)

[Tho86] J.A. Thornton, J. Vac. Sci. Technol. A 4(6), 3059 (1986)

[Tho89] J.A. Thornton, D.W. Hoffman, Thin Solid Films 171, 5 (1989)

[Tri96] J.-M. Triscone, L. Frauchiger, M. Decroux, L. Miéville, O. Fischer, C. Beeli, P. Stadelmann, G.-A. Racine, J. Appl. Phys. 79(8), 4298 (1996)

[Tro01] T. Trowbridge, N. Tam, M.-L. Chiang, G. Xing, and H.S. Joo, Enhanced Oxidation of Silicon Nitride using In Situ Steam Generation, $12-6^{\text {th }}$ Int. Sympos. On Silicon Nitride and Silicon Dioxide Thin Insulating Films, Washington, DC (2001)

[Tse01] J.H. Tseng, T.B. Wu, Appl. Phys. Lett. 78(12), 1721 (2001) 
[Wag33] C. Wagner, Beitrag zur Theorie des Anlaufvorganges. I-III, Z. Phys. Chem. B 21, 25 (1933)

[Wal89] F.W. Walker, J.R. Parrington, F. Feiner, Nuclides and Isotopes, Fourteenth Ed., General Electric Company, San Jose, California, 1989

[Wan96] Q. Wang, L. Gladfelter, D. Fennel Evans, Y. Fan, A. Franciosi, J. Vac. Sci. Tech. A 14(3), 747 (1996)

[web01] www.webelements.com/webelements/elements/text/O/isot.html (2001)

[Wer80] G.K. Wertheim, H.J. Guggenheim, Phys. Rev. B 22(10), 4680-4683 (1980)

[Wes89] W.D. Westwood, Handbook of plasma processing technology, S.M. Rossnagel, J.J. Cuomo, W.D. Westwood (Herausgeber), Noyes Publications, Park Ridge, New Jersey, USA (1989)

[Whi54] R.T.R. Whipple, Philos. Mag. 45, 1225 (1954)

[Wid88] D. Widmann, H. Mader, H. Friedrich, Technologie Hochintegrierter Schaltungen, Springer, München, 30 (1988)

[Win87] H. Windischmann, J. Appl. Phys. 62, 1800 (1987)

[Win91] H. Windischmann, J. Vac. Sci. Technol. A 9(4), 2431 (1991)

[Win92] Crit. Rev. In Sol. Stat. And Mat. Sci. 17, 547-596 (1992)

[Wut88] M. Wutz, H. Adam, W. Walcher, Theorie und Praxis der Vakuumtechnik, 4. Aufl., Vieweg, Braunschweig (1988)

[Yam97] S. Yamamichi et al., IEEE Trans. Electr. Dev. 44, 1076-1083 (1997)

[Yoo99] D.-S. Yoon, H.K. Baik, S.-M. Lee, S.-I. Lee, J. Vac. Sci. Technol. B 17(4), 1470-1476 (1999)

[Yu86] L.S: Yu, J.M.E. Harper, J.J. Cuomo, D.A. Smith, J. Vac. Sci. Technol. A 4(3), 443 (1986)

[Yun98] T. Yunogami, T. Kumihashi, Jpn. J. Appl. Phys. 37, 6934 (1998)

[Zha95] Y. Zhang, M. K. Jain, S. Nag, Advanced Metallization and Interconnect Systems for ULSI Applications, Portland, OR, 1-5 (1995)

[Zha99] F. Zhang, J.-S. Maa, S.T. Hsu, S. Ohnishi, W. Zhen, Jpn. J. Appl. Phys. 38, 14471449 (1999)

[Zhu98] S. Zhu, F. Wang, W. Wu, J. Appl. Phys. 84(11), 6399-6408 (1998)

[Zor94] G.M. Zorn, Analytical Application Note 337, Siemens AG (1994) 


\section{Danksagungen}

Mein ganz besonderer Dank gilt Herrn Dr. Kasko, Herrn Prof. Dr. Samwer, Herrn Dr. Geyer und Herrn Dr. Mikolajick, die durch ihre Hilfs- und Diskussionsbereitschaft während der Promotion mir immer unterstützend zur Seite gestanden haben. Als Betreuer haben sie durch ihr persönliches Engagement wesentlich zum Gelingen dieser Arbeit beigetragen. Weiterhin möchte ich den Verantwortlichen bzw. den Leitern des Ferro-Projekts, Herrn Dr. Klose, Frau Dr. Dehm und Herrn Dr. Scheibe für ihre Hilfsbereitschaft und die freundliche Integration ins Team danken.

Ganz besonders möchte ich mich ebenfalls bei allen Mitarbeitern des Ferro-Teams in München und in Shin-Sugita, insbesondere Herrn Dr. Nagel, Herrn Dr. Beitel, Herrn Dr. Bruchhaus, Herrn Dr. Röhner und Herrn Dr. Schindler bedanken. Sie haben ebenfalls einen großen Anteil zum Gelingen dieser Arbeit beigetragen. Für die nette Hilfe und Einweisung an der ENDURA möchte ich mich bei Herrn Dr. Wendt und Frau Heger bedanken, für die offenen Ohren und die freundliche Unterstützung bin ich dem AMAT-on-site-Service Team in München dankbar.

Für die gute Zusammenarbeit in München gilt Herrn Krönke und Herrn Jaschke, sowie den übrigen Doktoranden des Teams, Herrn Moert, Herrn Boubekeur und Herrn Bachhofer mein Dank, die mich in jeder Phase meiner Arbeit mental und organisatorisch viel unterstützt haben. Für das aufmerksame Korrekturlesen der Arbeit und hilfreiche Anregungen danke ich Herrn Dr. Damaschke und Frau Lücke.

Einen dreifachen Dank den Mitarbeitern des I. Physikalischen Instituts, besonders Herrn Streng und Herrn Becker für die nette Zeit und die urgemütliche, unvergleichlich gute Atmosphäre. Herr Mueller und Herr Harms haben es immer verstanden, die Arbeitslaune und Motivation auf einem überdurchschnittlichen Level zu halten. Für die Diskussions- und Hilfsbereitschaft möchte ich mich besonders bei Frau Dr. Schneider sowie Frau Gehrke bedanken.

Ganz besonders möchte ich mich bei Herrn Dr. Seibt für die erfolgreiche und freundliche Kooperation bei den TEM-Analysen bedanken. Weiterhin bin ich den Diplomanden und Doktoranden des IV. und II. Physikalischen Instituts für die guten Tipps und die Hilfsbereitschaft bei der Probenpräparation für das TEM großen Dank schuldig. Persönlich möchte ich mich bei Herrn Huang und Herrn Feldermann bedanken.

Für die freundliche Unterstützung, organisatorische Hilfe und die Bereitstellung von Experimentierplätzen bin ich den Mitarbeitern der Siemens AG, Abt. CT MM2, Herrn Primig, Frau Männer, Frau Preu und Herrn Kobusch ganz besonders dankbar. 
Mein ganz besonderer Dank gilt der Analytikgruppe CT MM7 der Siemens AG München, besonders Herrn von Criegern und Herrn Dr. Jahnel, sowie Frau Weidinger für die freundliche und hilfsbereite Zusammenarbeit bei den SIMS Analysen, sowie Herrn Dr. Scheithauer für die XPS Untersuchungen.

Weiterhin möchte ich Herrn Dr. Hösler und Frau Dr. Bleyl für die nette Unterstützung bei den AES und RBS Messungen danken. Der Röntgenanalytikabteilung - Herrn Dr. Schuster, Herrn Dr. Goebel und ganz besonders Frau Jobst - möchte ich für die Röntgen Analysen und ihre hilfreiche Diskussionsbereitschaft danken.

Für die Unterstützung bei den Rasterkraftmikroskopieanalysen und den REM Analysen möchte ich mich bei der Infineon Fehleranalyseabteilung (CFE FA5) bedanken.

Weiterhin möchte ich mich bei allen Infineon- und Toshiba Mitarbeitern in Shin-Sugita bedanken, die mir bei meinem Japan-Aufenthalt viel geholfen haben und mich in meiner Arbeit durch ihre stimulierenden Diskussionen weitergebracht haben. Meine ganz besondere Anerkennung gilt Herrn Dr. Moon, der mich mit seinem persönlichen Einsatz und seiner Hilfsbereitschaft sehr unterstützt hat.

Besonderer Dank gilt meiner Familie für ihre Unterstützung, ihre Hilfsbereitschaft und ihr Interesse an dieser Arbeit. 


\section{Lebenslauf}

Name: $\quad$ Cay-Uwe Pinnow

Geburtsdatum: $\quad$ 15.06.1974

Geburtsort: Kiel

Staatsangehörigkeit: deutsch

1980-1984 Grundschule Plön

1984-1993 Gymnasium Plön

$1993 \quad$ Abitur

1993-1994 Grundwehrdienst in Plön

1994-1999 Physikstudium an der Georg-August-Universität Göttingen

$1996 \quad$ Vordiplom in Physik

1998-1999 Diplomarbeit am I. Physikalischen Institut der Universität Göttingen bei Priv.-Doz. Dr. U. Geyer mit dem Thema „Analyse der Nanokristallisation von dünnen amorphen Ta-Si-N und Ti-Si-N Schichten“

1999

Diplom in Physik

$1999-2001$

Anfertigung der vorliegenden Dissertation am I. Physikalischen Institut der Universität Göttingen bei Prof. Dr. K. Samwer, Priv.-Doz. Dr. U. Geyer 